\title{
Aprimorando o Corretor Gramatical CoGrOO
}

William Daniel Colen de Moura Silva

DisSERTAÇÃo APRESENTADA

$\mathrm{AO}$

Instituto De Matemática e Estatística

DA

Universidade DE SÃo Paulo

PARA

OBTENÇÃO DO TÍTULO

$\mathrm{DE}$

Mestre em CiÊnCIAS

Programa: Mestrado em Ciência da Computação

Orientador: Prof. Dr. Marcelo Finger

Durante o desenvolvimento deste trabalho o autor recebeu auxílio financeiro do CNPq

São Paulo, 14 de janeiro 2013 


\section{Aprimorando o Corretor Gramatical CoGrOO}

Esta versão da dissertação contém as correções e alterações sugeridas pela Comissão Julgadora durante a defesa da versão original do trabalho, realizada em 06/03/2013. Uma cópia da versão original está disponível no Instituto de Matemática e Estatística da Universidade de São Paulo.

Comissão Julgadora:

- Prof. Dr. Marcelo Finger (orientador) - IME-USP

- Prof. Dr. Alfredo Goldman Vel Lejbman - IME-USP

- Prof. Dr. Marco Fernando Lopes - FFLCH-USP 


\section{Agradecimentos}

Este trabalho é fruto de meses de pesquisa e dedicação, cuja conclusão dependeu, direta e indiretamente, da colaboração de várias pessoas e instituições, que merecem meus agradecimentos.

Primeiramente, agradeço ao meu orientador, o Prof. Marcelo Finger, por acreditar no projeto, pelo seu empenho e por transmitir sua experiência prática e teórica que viabilizaram este trabalho.

À Profa. Graça Nunes (ICMC-USP), por disponibilizar o corpus PROBI e NILC, usados neste trabalho. Ao Prof. Marcos Lopes (FFLCH-USP) pelo apoio e por suas valiosas sugestões.

Agradeço a todos que contribuíram diretamente com o desenvolvimento do CoGrOO 4.0.0, especialmente ao Arthur Branco Costa pelo pela implementação da nova API e verificadores, pelo empenho no projeto Jspell.br, e pelas diversas discussões sobre particularidades da nossa gramática.

À equipe de Laboratório de Programação Extrema 2010 (Eduardo Katayama, Wesley Seidel Carvalho, Robson Santos Sousa, Filipe Manoel Xavier Resina, Thiago Maciel Batista, Carlos Eduardo Dantas de Menezes), e ao Michel Oleynik, que construíram o portal CoGrOO Comunidade, que hoje é um valioso canal para a participação da comunidade de usuários no desenvolvimento do projeto. E claro, agradeço a todos os usuários que reportaram erros no portal, colaborando com a construção do corpus Comunidade.

Ao Michel Oleynik, Wesley Seidel e Prof. Carlos Eduardo Dantas de Menezes, pelas diversas discussões sobre processamento de linguagem, software livre e correção gramatical, pelas revisões do texto e dicas.

Agradeço as instituições CNPq, pelo financiamento deste projeto. À USP por disponibilizar os recursos necessários para me dedicar à dissertação, em especial ao Departamento de Ciência da Computação do IME e ao Centro de Competência em Software Livre (CCSL).

Agradeço minha namorada, Giliane Rodrigues de Souza pelo incentivo e por toda sua paciência durante a minha ausência. Agradeço ainda todos meus familiares e amigos, que foram vitais durante todos estes anos de estudos.

Finalmente, e mais importante, agradeço meus pais, Deoclécio de Moura Silva e Edmea Colen de Moura Silva, e Barbara, minha irmã, pelo apoio e dedicação durante toda a minha vida. Sem tal os quais nada teria sido possível. 


\section{Resumo}

O CoGrOO é um corretor gramatical de código aberto em uso por milhares de usuários de uma popular suíte de escritório de código aberto. Ele é capaz de identificar erros como: colocação pronominal, concordância nominal, concordância sujeito-verbo, uso da crase, concordância nominal e verbal e outros erros comuns de escrita em Português do Brasil. Para tal, o CoGrOO realiza uma análise híbrida: inicialmente o texto é anotado usando técnicas estatísticas de Processamento de Linguagens Naturais e, em seguida, um sistema baseado em regras é responsável por identificar os possíveis erros gramaticais.

O objetivo deste trabalho é reduzir a quantidade de omissões e intervenções indevidas e, ao mesmo tempo, aumentar a quantidade de verdadeiros positivos sem, entretanto, adicionar novas regras de deteç̧ão de erros. A última avaliação científica do corretor gramatical foi realizada em 2006 e, desde então, não foram realizados estudos detalhados quanto ao seu desempenho, apesar de o código do sistema ter passado por substancial evolução. Este trabalho contribuirá com uma detalhada avaliação dos anotadores estatísticos e os resultados serão comparados com o estado da arte. Uma vez que os anotadores do CoGrOO estão disponíveis como software livre, melhorias nesses módulos gerarão boas alternativas a sistemas proprietários.

Palavras-chave: PLN, Software Livre, Corretor Gramatical. 


\section{Abstract}

CoGrOO is an open source Brazilian Portuguese grammar checker currently used by thousands of users of a popular open source office suite. It is capable of identifying Brazilian Portuguese mistakes such as pronoun placement, noun agreement, subject-verb agreement, usage of the accent stress marker, subject-verb agreement, and other common errors of Brazilian Portuguese writing. To accomplish this, it performs a hybrid analysis; initially it annotates the text using statistical Natural Language Processing (NLP) techniques, and then a rule-based check is performed to identify possible grammar errors.

The goal of this work is to reduce omissions and false alarms while improving true positives without adding new error rules. The last rigorous evaluation of the grammar checker was done in 2006 and since then there has been no detailed study on how it has been performing. This work will also contribute a detailed evaluation of low-level NLP modules and the results will be compared to state-of-the-art results. Since the low-level NLP modules are available as open source software, improvements on their performance will make them robust, free and ready-to-use alternatives for other systems.

Keywords: NLP, FLOSS, Grammar Checker. 


\section{Contents}

1 Introduction $\quad 1$

1.1 Problem Statement . . . . . . . . . . . . . . . . . 2

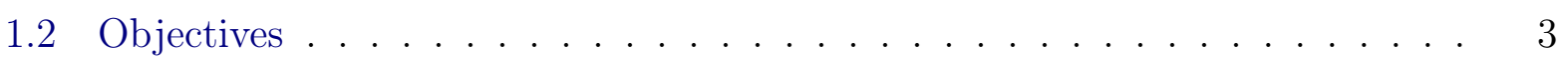

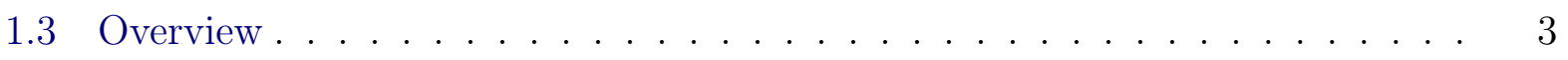

2 Background $\quad 5$

2.1 Natural Language Processing . . . . . . . . . . . . . . . 5

2.1.1 Statistical x Rule-Based Approach . . . . . . . . . . . . 6

2.1 .2 Corpora . . . . . . . . . . . . . . . . . 6

2.1 .3 Evaluation . . . . . . . . . . . . . . . 7

2.2 NLP Frameworks and Annotators . . . . . . . . . . . . . . . 8

2.2 .1 Brill .............................. 9

2.2 .2 VLMM Tagger . . . . . . . . . . . . . . . . 9

2.2 .3 Palavras . . . . . . . . . . . . . . . . . . . . . . 9 9

2.2 .4 LX-Group . . . . . . . . . . . . . . . . . . . 10

2.2.5 Apache OpenNLP Framework . . . . . . . . . . . . . . 10

2.3 Linguistic Resources . . . . . . . . . . . . . . . . . . . . . . . . . . 12

2.3.1 Dictionaries . . . . . . . . . . . . . . . . 12

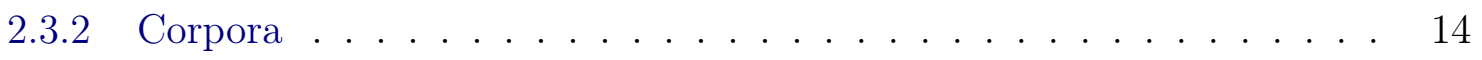

2.4 Proofreaders . . . . . . . . . . . . . . . . . . . . 16

2.4 .1 ReGra . . . . . . . . . . . . . . . . . . 16

2.4 .2 LanguageTool . . . . . . . . . . . . . . . . . . . . . . . . . . . . . . . . . . .

2.4.3 LightProof . . . . . . . . . . . . . . . . 17

3 CoGrOO - an Open Source Grammar Checker 19

3.1 Architecture . . . . . . . . . . . . . . . . . . 19

3.1.1 Sentence Boundary Detector . . . . . . . . . . . . . . 20

3.1.2 Tokenizer . . . . . . . . . . . . . . . . . . . . . 21

3.1.3 Post Tokenizer . . . . . . . . . . . . . . . . . . . . 23

3.1.4 Part-of-Speech Tagger . . . . . . . . . . . . . . . . 23

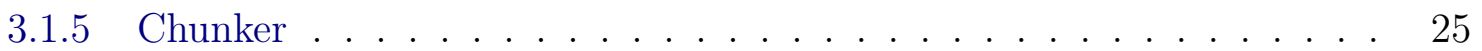

3.1.6 Shallow Parser . . . . . . . . . . . . . . . . 25 
3.1 .7 Grammar Error Detector . . . . . . . . . . . . . 26

3.2 Annotator Accuracy . . . . . . . . . . . . . . . . . . 27

3.2 .1 Discussion . . . . . . . . . . . . . . . . 27

3.3 CoGrOO UIMA . . . . . . . . . . . . . . . . . . . . . . . . . 28

3.4 CoGrOO Community . . . . . . . . . . . . . . . . . . 28

4 A new grammar checker proposal $\quad 31$

4.1 Apache OpenNLP 1.5.x . . . . . . . . . . . . . . . . . . . . 31

4.2 The New Software Architecture . . . . . . . . . . . . . . . . . . . 33

4.3 Experiments Plan . . . . . . . . . . . . . . . . . . . 36

4.4 Experiment Preparation . . . . . . . . . . . . . . . . . . . . 37

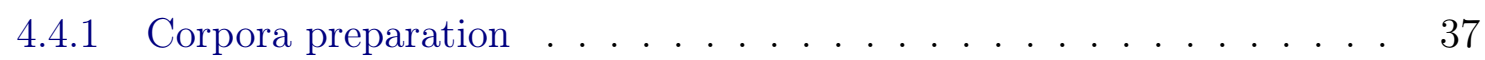

4.4 .2 10-Fold Cross-Validation Tool . . . . . . . . . . . . . . . 38

4.4 .3 Grammar Checker Evaluation Tool . . . . . . . . . . . . . . . . . 39

5 Initial Evaluation $\quad 43$

5.1 PROBI Corpus . . . . . . . . . . . . . . . . . . 43

5.2 Metro Corpus . . . . . . . . . . . . . . . . . . . 44

5.3 Bosque . . . . . . . . . . . . . . . . . . . . . . 44

5.4 CoGrOO Community Corpus . . . . . . . . . . . . . 45

5.5 Conclusion . . . . . . . . . . . . . . . . . . . . 46

$\begin{array}{lll}6 & \text { NLP Development and Evaluation } & 47\end{array}$

6.1 Code Changes . . . . . . . . . . . . . . . . . . . . . 47

6.1.1 Evaluation . . . . . . . . . . . . . ..... 48

6.1.2 Conclusion . . . . . . . . . . . . . . ..... 50

6.2 Sentence Boundary Detector . . . . . . . . . . . . . . . . 51

6.2.1 Default Contextual Predicates . . . . . . . . . . . . . . 51

6.2.2 Additional Context Predicates . . . . . . . . . . . . . . . . . 53

6.2.3 Previous Work . . . . . . . . . . . . . . . 56

6.2.4 Evaluating the Grammar Checker . . . . . . . . . . . . . 57

6.2 .5 Conclusion . . . . . . . . . . . . . . . . . . 61

6.3 Tokenizer . . . . . . . . . . . . . . . . . . . . . . 62

6.3.1 Default Contextual Predicates . . . . . . . . . . . . . 62

6.3 .2 Previous Work . . . . . . . . . . . . . . . 68

6.3.3 Evaluating the Grammar Checker . . . . . . . . . . . . . . . . . . . 69

6.3.4 Conclusion . . . . . . . . . . . . . . . . . . . . 73

6.4 Name Finder . . . . . . . . . . . . . . . . . . . . . . . . . . . 74

6.4.1 Default Contextual Predicates . . . . . . . . . . . . . . . 74

6.4 .2 Previous Work . . . . . . . . . . . . . . . . 77

6.4.3 Evaluating the Grammar Checker . . . . . . . . . . . . . 77 
6.4.4 Conclusion . . . . . . . . . . . . . . . 80

6.5 Contraction Finder . . . . . . . . . . . . . . . . . 81

6.5.1 Contextual Predicates . . . . . . . . . . . . . . . . 81

6.5.2 Previous Work . . . . . . . . . . . . . . . 83

6.5.3 Evaluating the Grammar Checker . . . . . . . . . . . . . 83

6.5.4 Conclusion. . . . . . . . . . . . . . 86

6.6 POS Tagger . . . . . . . . . . . . . . . . . . . 87

6.6.1 Multi-Word Expressions _. . . . . . . . . . . . . 87

6.6.2 Sequence Validation and the POS Dictionary . . . . . . . . . . . 87

6.6.3 Default Context Generator . . . . . . . . . . . . . . . . 88

6.6.4 Additional Context . . . . . . . . . . . . . . . . . . . 89

6.6.5 Custom Context Generator . . . . . . . . . . . . . . . . 93

6.6.6 Multi-Word Expressions Results . . . . . . . . . . . . . . 94

6.6.7 Previous Work . . . . . . . . . . . . . . . . . . . 95

6.6.8 Evaluating the Grammar Checker . . . . . . . . . . . . . . . 96

6.6.9 The Ambiguity of the Preposition A Versus the Article A . . . . . . . 101

6.6 .10 Conclusion . . . . . . . . . . . . . . . . . . . 101

6.7 Featurizer . . . . . . . . . . . . . . . . . . . . 103

6.7.1 Sequence Validation and the Feature Dictionary . . . . . . . . . . . . 103

6.7.2 Contextual Predicates . . . . . . . . . . . . . . . 103

6.7.3 Previous Work . . . . . . . . . . . . . . . . . . . . 107

6.7.4 Evaluating the Grammar Checker . . . . . . . . . . . . . . 107

6.7.5 Conclusion . . . . . . . . . . . . . . . 110

6.8 Chunker . . . . . . . . . . . . . . . . . . . . . . . 112

6.8.1 Contextual Predicates . . . . . . . . . . . . . . . . . . 112

6.8.2 Previous Work . . . . . . . . . . . . . . . . 115

6.8.3 Evaluating the Grammar Checker . . . . . . . . . . . . . 116

6.8.4 Conclusion . . . . . . . . . . . . . . . . . . . . . 117

6.9 Shallow Parser . . . . . . . . . . . . . . . . . . . . . . . . 119

6.9.1 Contextual Predicates . . . . . . . . . . . . . . . . 119

6.9 .2 The Multiple Clause Issue . . . . . . . . . . . . . . . . . . 120

6.9.3 Gender and Number of Subject and Predicate . . . . . . . . . . . . 121

6.9 .4 Rule Improvements . . . . . . . . . . . . . . . . . . . . . . . 122

6.9.5 Evaluating the Grammar Checker . . . . . . . . . . . . . . . 122

6.9.6 Conclusion . . . . . . . . . . . . . . 126

7 Final Evalutation $\quad 127$

7.1 PROBI Corpus . . . . . . . . . . . . . . . . 127

7.2 Metro Corpus . . . . . . . . . . . . . . . . . . 129

7.3 Bosque . . . . . . . . . . . . . . . . . . . 130 
7.4 CoGrOO Community Corpus . . . . . . . . . . . . . 130

7.5 Comparison to Other Systems . . . . . . . . . . . . . . . . . . 131

8 Conclusions $\quad 133$

8.1 Major Contributions . . . . . . . . . . . . . . . . 133

8.1.1 A More Effective Libre|OpenOffice Grammar Checker . . . . . . . . . 133

8.1.2 Identification of Key Issues in Training Apache OpenNLP Models for Portuguese . . . . . . . . . . . . . . . . . 134

8.1.3 Efficient Models for Portuguese Natural Language Processing . . . . . 135

8.1.4 Contributions to Open Source . . . . . . . . . . . . 136

8.1.5 Contributions to Other Research Projects . . . . . . . . . . 137

8.2 Future Studies . . . . . . . . . . . . . . . . . . . . . . 138

$\begin{array}{lr}\text { A Base POS Tagset } & 139\end{array}$

A.1 Class tags . . . . . . . . . . . . . . . . . . . . . . 139

A.2 Inflection Tags. . . . . . . . . . . . . . . . . 139

A.3 Chunk tags . . . . . . . . . . . . . . . . . . . . 139

A.4 Shallow Parser tags . . . . . . . . . . . . . . . . . . . 139

$\begin{array}{ll}\text { B PROBI Error Categories } & 143\end{array}$

$\begin{array}{lr}\text { C Source Code, Libraries and Tools } & 147\end{array}$

C.1 License . . . . . . . . . . . . . . . . . . . . . . . 147

C.2 Download the Source . . . . . . . . . . . . . . . . . 148

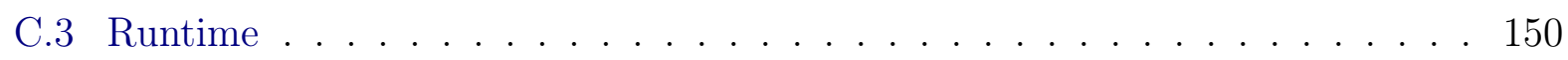

C.3.1 Build . . . . . . . . . . . . . . . . . . . 150

C.3.2 Executing from the Command Line . . . . . . . . . . . . 150

C.3.3 CoGrOO as a NLP Library . . . . . . . . . . . . . . . . . 151

C.3.4 CoGrOO as a Grammar Check Library . . . . . . . . . . . . . . . . 152

C.4 Evaluation . . . . . . . . . . . . . . . . . . . 154

C.4.1 Executing 10-Fold Cross-Validation . . . . . . . . . . . . . . . 154

C.4.2 Grammar Checker Evaluation . . . . . . . . . . . . . 156

$\begin{array}{ll}\text { Bibliography } & 163\end{array}$ 


\section{Chapter 1}

\section{Introduction}

Apache OpenOffice is a multi-platform and multilingual office suite, and an open source project $^{1}$. As with its sister project LibreOffice, it is the successor of the OpenOffice.org project, whose first release dates back to 2002. OpenOffice.org did not possess grammarchecking functionality, which made it less competitive compared with other non-open source alternatives. This motivated some NLP researchers to create $\mathrm{CoGrOO}^{2}$, a Brazilian Portuguese grammar checker, a project initially sponsored by FINEP (a Research and Projects Funding agency). This research on CoGrOO began in 2004, and since its first release in 2006 it has been adopted by important companies like Petrobras - the biggest company in Brazil and the 8th biggest in the world in market value - and Celepar - the Paraná State information technology company, responsible for deploying software for government offices and public schools. CoGrOO accumulated over a hundred thousand downloads from its official website.

CoGrOO is an open source project. It is capable of identifying Portuguese mistakes like pronoun placement, noun agreement, subject-verb agreement, usage of the accent stress marker ( '), and other common errors found in Brazilian Portuguese writing.

The CoGrOO grammar checker takes the user's text as input, and outputs a list of possible errors. To accomplish this, it performs a shallow parsing followed by rule-based checking. Initially it analyzes the text using Natural Language Processing (NLP) techniques. The text goes through a pipeline of annotators to identify sentence and token boundaries, to assign a part-of-speech (POS) tag (class and inflection tags, see Appendix A) for each word and to find phrase chunks and subject-verb relationships. Then it matches a set of man-made error rules with the resultant structure. An error rule consists of a pattern of words, POS tags, or phrase tags, and some other information, like a description of the error and how an alternative suggestion can be generated.

Although CoGrOO is a successful project considering the number of downloads, which

\footnotetext{
${ }^{1}$ Apache OpenOffice - Free and Open Productivity suite, http://openoffice.org, last accessed on $12 / 10 / 2012$

${ }^{2}$ CoGrOO official website, http://cogroo.org, last accessed on 12/10/2012
} 
already surpasses 170 thousands $^{3}$, its internal components could benefit from several improvements. The main contribution of this work is an analysis of each module of the grammar checker (e.g. the sentence detector, tokenizer, POS tagger, chunker, shallow parser) to determine possible effectiveness bottlenecks and proposed changes to overcome them. No new error rules will be created to improve coverage; this work focuses only on the statistical modules of CoGrOO. Expanding the number of error rules will be left for future studies.

To validate the software against false errors, the grammar checker was tested using the reviewed part of the Floresta corpus (Afonso, 2003), which is extracted from newspaper articles. Reviewed texts are expected to have very few grammar errors. Coverage will be checked with 3 corpora: a) a new corpus assembled from real texts submitted by CoGrOO users; b) the Metro corpus (Menezes et al., 2006), composed of authentic texts with manually annotated grammar errors; and c) the PROBI corpus (Martins, 2002), composed of both correct and incorrect sentences, assembled to evaluate ReGra, a proprietary Brazilian Portuguese grammar checker.

\subsection{Problem Statement}

A written text is subject to errors such as (Kinoshita et al., 2006):

- Spelling errors: when a word is misspelled, for example as in "sugeito", while the correct spelling is "sujeito" (subject);

- Grammatical errors: when grammatical rules are not observed, for example in Nós vai para casa. (We goes home). Such errors relate to subject-verb agreement.

Automatic proofreaders are useful to help write documents with fewer mistakes. Currently, CoGrOO only handles grammar errors. In other words, it checks if the text complies with grammar rules. Spelling errors are not covered because most text processors include this functionality.

The grammar-checking task is difficult and complex. There is no established best strategy for assembling this kind of application. It requires a number of dictionaries and language models, and it must be computationally efficient. The grammar checker is a secondary tool in a text processor, and thus should not comprise much computational resources and affect the performance of its host system.

A major difficulty of automatic proofreading, as in other NLP tasks, lies in the handling of ambiguity. This problem arises in many stages of processing, from sentence segmentation to part-of-speech tagging. An example of ambiguity encountered while performing sentence boundary detection is to determine when a period indicates the end of a sentence or an

\footnotetext{
${ }^{3} \mathrm{CoGrOO}$ downloads count from Source Forge, http://sourceforge.net/projects/cogroo/files/stats/ timeline?dates $=2006-11-13+$ to $+2012-12-10$, last accessed on $12 / 10 / 2012$
} 
abbreviation. In part-of-speech tagging, an example is tagging the word "casa". In Portuguese it can be a noun meaning "home" or a verb meaning "to marry" according to the context:

- Nada como voltar para casa. (Nothing like coming home.);

- Ele se casa na semana que vem. (He gets married next week.);

In the first sentence "casa" is a noun, while in the second it is a verb. In a text that may contain grammar errors, this analysis can be even more difficult to discern, because the text does not follow the conventional grammar structure, and it was constructed in an unpredictable manner (Naber, 2003).

Grammar checker effectiveness can be evaluated by measuring the number of false alarms; that is, when the grammar checker points out an error where there is none, versus the number of correctly pointed out errors. False alarms are known as false positives and the fewer the better. Correctly pointed out errors are known as true positives, and the higher the better. More details about evaluation in Section 2.1.3

\subsection{Objectives}

The main goal of this work is:

To increase the number of true positives and decrease the number of false positives, without creating any new error rule.

Secondary aims are:

- To evaluate each module of the grammar checker to find bottlenecks;

- To improve low effectiveness modules;

- To evaluate the impact of the changes, in low-level and in the overall functionality;

- To increase quality of the Free/Libre/Open-Source Software (FLOSS) grammar checker for Portuguese;

- To increase quality of FLOSS NLP annotators for Portuguese.

\subsection{Overview}

Chapter 2 consists of a literature review covering natural language processing, followed by an analysis of existing software and language resources. Chapter 3 covers the current version of CoGrOO, including an architecture review. Chapter 4 proposes the new grammar checker version, as well as the development and evaluation plans. Chapter 5 presents an effectiveness evaluation of the current version of the grammar checker, followed by a code review and preliminary improvements and evaluation. Chapter 6 describes the changes in the pipeline and its modules, as well as evaluates the effectiveness of the improvements. Chapter 
7 presents the final effectiveness evaluation of the grammar checker, comparing the results with the initial evaluation and others grammar checkers. Finally, Chapter 8 summarizes the work by introducing its most important contributions and future work. 


\section{Chapter 2}

\section{Background}

The task of proofreading incorporates concepts from theoretical and applied sciences including natural language processing, statistics, search algorithms, and software engineering. This section provides a review of natural language processing and automatic proofreading, the state of the art, and the foundation of the field.

\subsection{Natural Language Processing}

Natural Language Processing (NLP) is linked to the field of Artificial Intelligence (AI) and Linguistics. As other AI areas, NLP attempts to emulate a human capability, in this case processing and generating a natural language. Although the task of understanding natural language is difficult or even nearly impossible to accomplish, there are many very useful smaller systems that could be improved, such as:

- Language generation, for example a converter from data in a computer database to human language;

- Language understanding, for example, converting from human language to data in a computer database;

- Automatic translation between different languages;

- Speech to text recognition, for example to dial numbers on a cell phone;

- Sentiment analysis, for example to determine if a review is positive or negative;

- Automatic grammar checking.

NLP systems can be highly complex. It is a common practice to break down systems into smaller "low-level" tasks, but this still presents non-trivial challenges (Silva, 2007).

Examples of these tasks are: sentence segmentation, tokenization, POS tagging, chunking, parsing, named entity recognition, coreference resolution, and others. Each task can be addressed using well-known technologies, such as finite-state automata, rule systems, logics, probabilistic models and machine learning (Jurafsky et al., 2000). 


\subsubsection{Statistical x Rule-Based Approach}

There is a division in respect to which statistical or rule-based approach is better for dealing with NLP systems. In rule-based approaches, a human writes instructions on how to process the information. For instance, it is easy to create rules in the form of a finite state automaton to tokenize a sentence. It is also possible, but not easy, to write rules to parse a sentence, as was done in Palavras (Section 2.2.3) using Constraint Grammar (Karlsson et al., 1995), or ReGra (Section 2.4.1) using augmented transition networks (Woods, 1970).

With processing power increasing yearly and the vast amount of electronic corpora available, the statistical approach has become popular. Statistical models can be created by extracting information from a language corpus (Section 2.1.2) and applying machine learning (ML) techniques. The advantage of this approach resides in the fact that it is not necessary to produce hand-written rules to model the language, as the ML algorithms can do it almost in a language independent manner. Examples of ML algorithms applied to NLP are Hidden Markov Models (HMM), N-Gram and Maximum Entropy.

Comparing both strategies, the advantage of a rule-based approach is that it allows for dealing with specificities of the language, and attain close to $100 \%$ accuracy; for example the Palavras parser (Bick, 2000) claims to have an accuracy of more than $99 \%$ in POS tagging; on the other hand, it is time-consuming arduous task to transcribe the rules and manage them. A statistical model is easier to assemble, because most of the work is done by computer; on the other hand, a state-of-the-art statistical POS tagger can reach at best 97\% accuracy (Silva, 2007).

\subsubsection{Corpora}

A corpus, the Latin word for "body", is a collection of texts representing some form of usage of a language (Manning and Schütze, 1999). A corpus is useful for both computer scientists and linguistics. A linguist may use it to measure usage of terms and constructions, and to compare how the language has changed using a historical corpus. On the other hand, a computer scientist may use a corpus to extract structures of the language, and to compute statistics to create language models.

An annotated corpus is a special type of linguistically marked-up corpus. For example, in PROBI corpus (Section 2.3.2) a sentence has annotations declaring if it has grammar errors, and if it does, it specifies the kind of error. The Bosque corpus (Section 2.3.2) has annotations for sentence segmentation, tokenization, POS tagging and parsing.

The main challenge is to find the right corpus one needs. For instance, Bosque has all the information needed to train statistical models, but on the other hand, it was assembled from texts extracted from newspapers from 1994. It is a very specific kind of text, and probability models created from it may not perform successfully while analyzing an essay written in 2011. For example, Oleynik et al. (2010) evaluated the same POS tagger trained with corpora from different domains to annotate Brazilian hospital discharge summaries. 
The results of training it using a newspaper corpus was only $75.3 \%$ accuracy, while training it with a corpus from the medical domain could reach $91.5 \%$ accuracy.

\subsubsection{Evaluation}

The most important measure of how well a system performs is to check it against what it was proposed to do. In the context of grammar checking for example, we expect it to correctly identify grammar errors. However, it is often necessary to create well-defined metrics to compare a system with another or to evaluate low-level component performance.

According to Manning and Schütze (1999), the evaluation strategy for NLP was taken from the area of Information Retrieval, especially the notions of precision and recall. They define a large collection of items, for example documents or sentences, and two concepts: target, a subset of the collection that matches a criterion, like relevant documents or sentences with grammar errors; and selected, the set of items that the system determines should match a given criterion. In the case of a grammar checker, the selected set would be the sentences it outputs as having errors and the target the sentences that a specialist annotated as having errors.

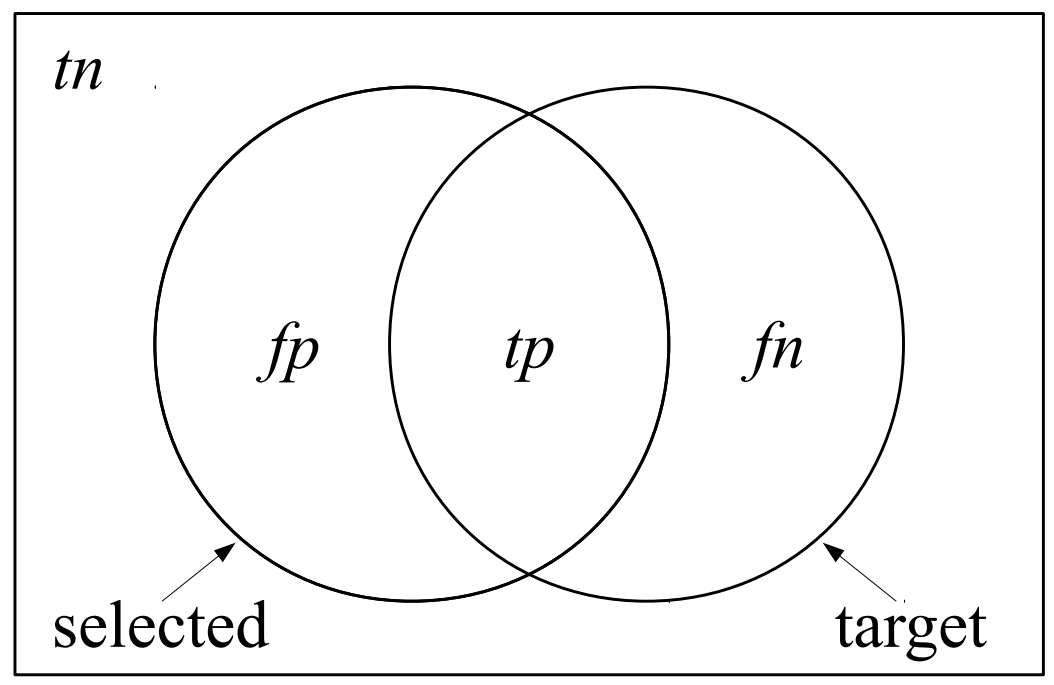

Figure 2.1: Motivating the measures of precision and recall. The rectangular area represents the full collection. The circles represent target items, and selected items. The captions show regions whose items represent true and false positives and true and false negatives in terms of target set and selected set. Precision is $\mathrm{tp} / \mid$ selected $\mid$, the proportion of target items in the selected set. Recall is $\mathrm{tp} / \mid$ target $\mid$, the proportion of target items that were selected.

True positives $(t p)$ occur when the system selected an item from the target, and false positives $(f p)$ occur when the system selected an item that was not in the target. Similarly, false negatives $(f n)$ occur when the system misses an item from target, and true negatives $(t n)$ occur when the system correctly excludes an item that was not in the target. In this respect, $t p$ and $t n$ are cases where the system got it right, and so they should be maximized. 
Precision is defined as the proportion of selected items that the system correctly marked:

$$
\text { precision }=\frac{t p}{t p+f p}
$$

Recall is defined as the proportion of target items that the system selected:

$$
\text { recall }=\frac{t p}{t p+f n}
$$

Depending on the application, it is important to minimize the number of $f p$. This is the case of grammar checking, where each $f p$ represents an incorrect intervention. For this reason, it is convenient to combine precision and recall into a single measure of overall performance. One way to do this is with the F-measure defined as follows:

$$
F=\frac{1}{\alpha \frac{1}{P}+(1-\alpha) \frac{1}{R}}
$$

where $P$ is precision, $R$ is recall and $\alpha$ is a factor which determines the weighting of precision and recall. With a value of $\alpha=0.5$, which evenly weights precision and recall, the F-measure is simplified to:

$$
F_{1}=\frac{2 P R}{R+P}
$$

The $F$-measure when $\alpha=0.5$ is known as $F_{1}$.

Accuracy is another term used regarding measuring the number of things the system got right: $\frac{t p+f n}{\text { all }}$. Accuracy only makes sense in some contexts, for example when categorizing all collection items, as a POS tagger that should assign tags to every token.

\section{N-Fold Cross-Validation}

$N$-fold cross-validation consists of splitting the data into $n$ parts; one part is used as the validation set and the training is performed with the other $n-1$ parts. This process is repeated $n$ times using each of the other $n-1$ parts as a validation set. The $n$ results from the folds are averaged to produce a single estimation. A 10-fold cross-validation is commonly used.

\subsection{NLP Frameworks and Annotators}

This section covers selected frameworks for NLP which are crelated to this work. It is a review of the available technologies. The selected annotators are Brill (Brill, 1992), VLMM (Kepler, 2005) and Palavras (Bick, 2000). The former is a popular POS tagger used in the FLOSS grammar checker LanguageTool (Section 2.4.2); this tagger was studied to investigate a CoGrOO competitor technology. The second is another POS tagger designed to 
tackle Portuguese language specificities. The latter one, Palavras, is included here because of its importance for Portuguese: its output was used as annotation in Floresta Sintá(c)tica corpora (Section 2.3.2), the corpora using to train CoGrOO.

The selected frameworks are LX-Group and OpenNLP. The first one is a set of tools for European Portuguese. It is described here because of the documentation it provides, detailing how developers handled peculiarities of the language. OpenNLP Framework is used in the current version of CoGrOO, and because of that it is described in greater detail in Section 2.2 .5 .

\subsubsection{Brill}

The POS tagger developed by Eric Brill (1992) is a hybrid in the sense that it uses both statistical and rule-based approaches. An advantage is that the algorithm can learn the rules automatically using a corpus. When performing classification of a word, it will consider all possible tags, but will initially assign the most common for a word without considering context. The algorithm compares the predicted tag with the real one, and tries to come up with rules based on the context (called patches), which repair these errors. With very few of these automatically constructed rules the system achieves $97 \%$ accuracy.

The Brill method, originally developed for English, cannot be applied directly in Portuguese texts because of its richer morphology. A variant described by Finger and Alves (1999) adapts the method for Brazilian Portuguese and has achieved 95.45\% accuracy.

\subsubsection{VLMM Tagger}

The statistical POS tagger developed by Kepler (2005) applies the recent theory of Variable Length Markov Model. The parameters of this tagger were fine-tuned to tackle specificities of Portuguese, and since it is probabilistic, it does not depend on any special tag set or corpus. During his work, Kepler trained and evaluated his tagger using a historical corpus, Tycho Brahe (Galves and Faria, 2010), and achieved 96.67\% accuracy.

\subsubsection{Palavras}

The Palavras parser was developed by Bick (2000) and is available as a Web application at the VISL project site ${ }^{1}$, but cannot be downloaded and is not available as FLOSS. Palavras was used to automatically annotate Floresta Sintá(c)tica corpora (Section 2.3.2).

Palavras is a rule system created using a Constraint Grammar (Karlsson et al., 1995) framework. It claims to have an accuracy of more than $99 \%$ on POS tagging, and above 98\% on syntax, making Palavras a state-of-the-art parser for Portuguese as well as other languages (Bick, 2000).

\footnotetext{
${ }^{1}$ VISL project site: http://visl.sdu.dk, last accessed on 11/23/2011
} 


\subsubsection{LX-Group}

In "Shallow Processing of Portuguese", Silva (2007) describes creating a set of low-level NLP tools for European Portuguese. The results of his work, together with contributions of other researchers of the Natural Language and Speech Group at the University of Lisbon, is the LX-Center (LX-CENTER, 2011).

The tasks covered by Silva include Sentence Segmentation, Tokenization, Part-of-Speech Tagging, Nominal Featurization and Nominal Lemmatization. His research details the main challenges of dealing with NLP in Portuguese, and the proposed solutions to each problem. Often Silva used machine-learning techniques, training and evaluating them using a 260.000token corpus created and provided by the Center of Linguistics at the University of Lisbon.

Each of the performance results obtained is compared to results obtained by other groups in different languages. Silva claims that the results yield a state-of-the-art performance or, in some cases, an advanced state-of-the-art performance.

\subsubsection{Apache OpenNLP Framework}

The Apache OpenNLP² (Baldridge et al., 2002) is an NLP framework that includes a set of ready-to-use tools for NLP tasks designed on top of ML algorithms like MaxEnt and Perceptron. Since version 2.0, CoGrOO has relied on OpenNLP, as described on Section 3.1.

Due to the extensive usage of this framework in CoGrOO, the following sections delve deeper into some concepts of the tool, especially the concepts behind Maximum Entropy and Perceptron algorithms, as well as the ideas behind feature extraction, contextual predicates and sequence validators.

\section{The Maximum Entropy Algorithm}

The CoGrOO statistical NLP modules rely on Apache OpenNLP with the Maximum Entropy Framework (Maxent). The method has the advantage of being quite flexible in the features that can be incorporated in the model. This section briefly explains the concepts relevant to this theory.

Ratnaparkhi (1996) proposed the usage of the Principle of Maximum Entropy (MaxEnt) (Jaynes, 1957) models in NLP. The product is a statistically generated language model. The proposed algorithm works as follows (Jurafsky et al., 2000):

1. Initially it makes no assumptions about the text. All possibilities have equal chances - the entropy is maximum;

2. A new feature is added representing a characteristic of the sample text retrieved from the corpus. This feature changes the likelihood of each tag;

3. The maximum entropy of the system is estimated again;

\footnotetext{
${ }^{2}$ I contribute to OpenNLP since 2009, and since $2010 \mathrm{I}$ am an active committer. Apache OpenNLP project team: http://opennlp.apache.org/team.htmlhttp://visl.sdu.dk, last accessed on 12/10/2012
} 
4. New features are added until the system is very close to the sample text.

The following example shows the idea behind the algorithm. Imagine someone wants to find a POS tag for the word casa.

Initially it produces an equiprobable distribution of probabilities, which makes the fewest assumptions (see tagset in Appendix A):

\begin{tabular}{|c|c|c|c|c|c|c|c|c|c|c|c|}
\hline $\mathrm{N}$ & PROP & SPEC & DET & PERS & ADJ & ADV & V & NUM & PRP & KS & $\ldots$ \\
\hline \hline $1 / 14$ & $1 / 14$ & $1 / 14$ & $1 / 14$ & $1 / 14$ & $1 / 14$ & $1 / 14$ & $1 / 14$ & $1 / 14$ & $1 / 14$ & $1 / 14$ & $\ldots$ \\
\hline
\end{tabular}

With access to training data, one can analyze it and discover, for instance, that the only possible classes for the word are: N, PROP, ADJ and V. So a new constraint (2.5) could be added and it updates the probabilities:

$$
\begin{gathered}
\begin{array}{|c|c|c|c|}
\hline \mathrm{N} & \text { PROP } & \text { ADJ } & \mathrm{V} \\
\hline \hline 1 / 4 & 1 / 4 & 1 / 4 & 1 / 4 \\
P(N)+P(P R O P)+P(A D J)+P(V)=1
\end{array}
\end{gathered}
$$

Again from the training data, one discovered that for each 10 occurrences of this word, 8 times it was classified as noun or proper noun. Another constraint was added (2.6) and the probabilities were updated:

$$
\begin{array}{|c|c|c|c|}
\hline \mathrm{N} & \text { PROP } & \text { ADJ } & \mathrm{V} \\
\hline \hline 4 / 10 & 4 / 10 & 1 / 10 & 1 / 10 \\
\hline
\end{array}
$$

If there was no further information, this would be the model that makes the fewest assumptions with what we know. There is nothing more about the word, but one discovered from the training data that for each 20 words, not only casa, one is a verb. With this information a new constraint could be added (2.7), updating the resultant language model as:

\begin{tabular}{c|c|c|c|}
\hline $\mathrm{N}$ & PROP & ADJ & $\mathrm{V}$ \\
\hline \hline $4 / 10$ & $4 / 10$ & $3 / 20$ & $1 / 20$ \\
\hline \multicolumn{4}{c}{} \\
$P(V)=1 / 20$
\end{tabular}

This is the final model, which takes all available information into account and in which entropy is maximum. 


\section{The Perceptron Algorithm}

Although the popularity of the Maxent algorithm, recent theoretical and experimental results highlighted problems with the parameter estimation method (Collins, 2002). More specifically, the number of elements in the sample space exponentially increases with the number of features, while the number of distributions increases with the double exponential, making it harder to find the one with minimum entropy.

Collins (2002) introduced a way of using the same feature structure of Maxent in an algorithm that relies on Viterbi decoding of training examples, combined with simple updates. Collins proved that the algorithm is consistently more effective compared to Maxent in at least the tagging and chunking tasks.

\section{$2.3 \quad$ Linguistic Resources}

The linguistic resources required for this project are lexical dictionaries and corpora.

The lexical dictionaries are used to support the POS tagging task, for example, by restricting which tags should be assigned to tokens. The JSpell (Almeida and Pinto, 1994) dictionary is an example of a lexical dictionary because its entries include word class and inflection information.

The corpora are used both for training statistical modules and evaluating the effectiveness of the system. The annotated corpora from Floresta Sintá(c)tica (Afonso, 2003) can be used to train and evaluate the annotators, like the POS Tagger. The Bosque corpus is a subset of Floresta Sintá(c)tica, whose annotations are reviewed, and should be used when the quality of the annotations are more important than the quantity. Floresta Virgem and Amazônia, also subsets of Floresta Sintá(c)tica corpora, are larger, and should be used if quantity proves to be important.

PROBI, Metrô, Bosque and CoGrOO Community corpora will be used to evaluate the grammar checker effectiveness. Bosque is composed of revised texts and is useful to search for false positives, while PROBI and Metrô are annotated with grammar errors and can be used to evaluate the precision and recall of the system. Finally the CoGrOO Community corpus will be used for a final evaluation of the system.

Table 2.1 summarizes the available corpora for effectiveness evaluation and Table 2.2 summarizes the available corpora for training and evaluating statistical modules.

The following sections describe each available linguistic resource.

\subsubsection{Dictionaries}

\section{JSpell}

JSpell is a morphological analysis tool created by the Natura Project (Almeida, 2011). It includes a high quality lexical dictionary for European Portuguese, with information about 


\begin{tabular}{c|c|c|c} 
& Sentences & Grammar errors & Categories covered \\
\hline PROBI & 11,625 & 2,616 & All \\
\hline Metrô & 781 & 53 & adv, aha, con, cov, cra, ger, ptn \\
\hline Bosque & 4,212 & 0 & none \\
\hline CoGrOO Community & 457 & 188 & -
\end{tabular}

Table 2.1: Summary of available corpora for effectiveness evaluation. Error categories are described in Appendix B.

\begin{tabular}{c|c|c|c|c|c} 
& Sentences & POS Tags & Syntactic Tags & Proofread & Reviewed \\
\hline Floresta Virgem & 96,000 & yes & yes & yes & no \\
\hline Bosque & 4,212 & yes & yes & yes & yes \\
\hline Amazonia & 275,000 & yes & yes & no & no \\
\hline CETENFolha Cortado & $1,159,520$ & yes & no & no & no
\end{tabular}

Table 2.2: Summary of the available corpora to train and evaluate statistical modules.

word class and inflection. Two versions of the dictionary are available: the first includes changes for the Portuguese Language Orthographic Agreement of 1990 (OA) (Chiarelli et al., 1990), the second, the European variation before the OA.

As far as is known, there is no other open resource like JSpell for Portuguese. Vero has a lot more entries, and is the Brazilian Portuguese variant, but does not include morphological information. The OA version of JSpell can be extremely helpful, since with the OA, all Portuguese-speaking countries have standardized their vocabulary. The problem is that even with the OA, there are lots of terms that differ in the Brazilian and European variants, for example the term ecrã from the European variant does not exist in the Brazilian variant, but can be translated as tela (a type of screen).

\section{JSpell.br}

Jspell.br is an initiative of the CoGrOO team and its contributors which objective is to translate the JSpell dictionary to the Brazilian variant of Portuguese. The work is currently under development, but the dictionaries are already available and can be used by CoGrOO modules. Both dictionary and sources are available online ${ }^{3}$.

\section{Vero}

Vero is LibreOffice's spell checker (Moura, 2011). It includes a huge list of Brazilian Portuguese words. Vero is based on Hunspell (Nemeth, 2011a), which can be integrated with other programs. Hunspell can perform morphological analysis, but it does not work with Vero because it does not include morphological information.

\footnotetext{
${ }^{3}$ JSpell.br website, http://github.com/cogroo/jspell.br, last accessed on 12/10/2012
} 


\subsubsection{Corpora}

\section{Floresta Sintá(c)tica}

Floresta Sintá(c)tica (Syntactic Forest) is a publicly available treebank for Portuguese, created as a collaboration project between the VISL project ${ }^{4}$ and Linguateca ${ }^{5}$.

The annotated corpora are based on the output of the PALAVRAS parser (Section 2.2.3). The Floresta treebank has several copora, and some of them are listed in the following subsections.

Floresta Virgem Floresta Virgem (Virgin Forest) is a set of trees automatically created from the Constraint Grammar (CG) output of the PALAVRAS parser ${ }^{6}$. It is a 1,640,000word, 96,000-sentence corpus, with both Brazilian and European variants of Portuguese. The Brazilian portion is called the CETENFolha, and is a subset of the NILC Corpus. Its text material comes from the Folha de São Paulo newspaper. The European portion is called CETENPúblico, and its text material comes from the Público newspaper. This corpus is useful for training and test modules when quantity is better than quality.

Bosque Bosque (Grove) is a subset of Floresta Virgem, with fully revised annotations of 186,000 words and 9,368 sentences, of which 4,212 sentences are from CETENFolha, the Brazilian portion. It is available in flat CG format, or as trees in Árvores Deitadas format (Lying Trees) (AD) (Afonso, 2006) according to the Floresta Symbolset ${ }^{7}$. From AD format, it is easier to extract structural information, like chunks and clauses. This corpus is useful to train and test modules when quality is better than quantity.

Amazônia The Amazônia corpus was automatically annotated from the output of the PALAVRAS parser from the Brazilian cultural blog site Overmundo ${ }^{8}$, not revised, with 4,580,000 words. The site includes a large number of authors, from different parts of Brazil, which also reflects different writing styles. All texts are written in Brazilian Portuguese. An interesting feature of the Amazônia corpus is its named entities annotation, following the Harem $^{9}$ categories.

\footnotetext{
${ }^{4}$ Visual Interactive Syntax Learning: http://visl.sdu.dk, last accessed on 12/10/2012

${ }^{5}$ Linguateca project http://www.linguateca.pt, last accessed on 12/10/2012

${ }^{6}$ The Constraint Grammar category set of Palavras http://beta.visl.sdu.dk/visl/pt/info/portsymbol. html, last accessed on $12 / 15 / 2012$

${ }^{7}$ Grammatical categories (tags) used in the Floresta project http://beta.visl.sdu.dk/visl/pt/info/ symbolset-floresta.html, last accessed on $12 / 15 / 2012$

${ }^{8}$ Overmundo, a collaborative blog site http://www.overmundo.com.br/, last accessed on 12/10/2012

${ }^{9}$ HAREM: Reconhecimento de entidades mencionadas em português (Named Entity Recognition in Portuguese) http://www.linguateca.pt/HAREM, last accessed on 12/10/2012
} 


\section{CETENFolha Cortado}

CETENFolha Cortado (Cut) is a preprocessed portion of the Brazilian part of Floresta Virgem corpus. This corpus was created to train CoGrOO 1.0, and was used until the CoGrOO version 3.1 was introduced. The characteristics of the corpus are:

- Only sentences with less than 40 words;

- No sentences with spelling errors, sentences were spell checked;

- Numbers were substituted by placeholders;

- Cleaner format, with one sentence per line, and simplified annotation including only word class, inflection tags and lemma.

Although this format was interesting for CoGrOO 1.0, there were no clear advantages for using it on version 2.0 and above, but it was still used for the sake of simplicity.

\section{PROBI}

The PROBI Corpus (Martins, 2002) was developed to evaluate the ReGra grammar checker. It is composed of sentences supposedly verifiable in the written record of Brazilian Portuguese users. The corpus provides:

- a set of correct and legitimate sentences;

- a set of sentences which, although belonging to dialectal varieties of Brazilian Portuguese and practiced by a significant subset of native speakers, are not considered standard Portuguese nor recommended or tolerated by dictionaries and grammars;

- a set of sentences with typo or editing mistakes that do not belong to any variety of Portuguese.

PROBI aims to collect writing errors that a native speaker of Brazilian Portuguese could make. The correct and legitimate sentences were extracted from textbooks, writing manuals, dictionaries and other compilations. The incorrect sentences were collected from actual texts, produced by speakers of Portuguese, and some sentences were created to test some specific rules of ReGra. The corpus is annotated, and sentences with mistakes are categorized according to their deviations.

\section{Metrô Corpus}

The Metrô corpus was assembled to test the CoGrOO 1.0 grammar checker (Menezes et al., 2006). The texts were extracted from the São Paulo subway company's institutional website (Metro, 2011), in December 2006. The grammar errors in the text were manually annotated. The corpus was used to evaluate CoGrOO and compare the results with the ReGra grammar checker. 


\section{CoGrOO Community Corpus}

\section{4}

CoGrOO Community (Section 3.4) is a web application that provides collaborative tools for grammar-checker users. Users of $\mathrm{CoGrOO}$ were invited to join the Community portal and submit sentences that caused CoGrOO to fail, indicating whether it is a false negative or false positive. In each case the user provides relevant classification details. This error list will be used to evaluate the types of errors that appear most frequently for users, and will be part of a new corpus that also could be used to evaluate CoGrOO improvements.

\subsection{Proofreaders}

This section covers two popular automatic proofreaders. a) ReGra is a proprietary grammar checker for Brazilian Portuguese, embedded in Brazilian Portuguese version of Microsoft Office. It was developed in an academic environment, so it is well documented, but as source code, we can only test it in Microsoft Word; b) LanguageTool is an open source multi language grammar checker, but does not work with Portuguese texts. CoGrOO will be analyzed in a separate section.

\subsubsection{ReGra}

One of the first and most important efforts to create a Brazilian Portuguese proofreader was the ReGra project, the result of the partnership between the University of São Paulo and Itautec/Philco (Nunes and Oliveira, 2000). One of the most important benefits of the project was the creation of the Interinstitutional Center for Computational Linguistics (NILC), which plays an important role in the NLP community for Portuguese. They were responsible for assembling important lexical dictionaries and Corpora.

ReGra was one of the first grammar checkers for Brazilian Portuguese and, since 2000, Regra has been bundled with Microsoft Office suite. It is composed of three modules: statistical, mechanic and grammatical.

The statistical module performs calculations to compute parameters like the number of paragraphs, sentences, words and characters. It also executes the Flesch readability test (Flesch, 1948) adapted to Portuguese.

The mechanical module searches for typographical errors. It checks for repeated words, correct usage of spacing before and after a punctuation mark, correct use of case and parentheses balance.

The grammatical module is divided into two different approaches. The first one is an error-driven approach, which creates an Augmented Transition Network (Woods, 1970) to detect a chosen error. The advantage resides on being fast and easy to implement, but not being general. The second approach involves syntactical analysis to check deviations in the relations between different parts of the sentence. 
To evaluate ReGra, NILC created two corpora: the NILC Corpus and PROBI (Section 2.3.2). The evaluation consists of measuring the number of omissions, false errors and correct matches. Omissions and false errors should be minimized, while matches maximized.

\subsubsection{LanguageTool}

LanguageTool is an Open Source grammar checker available in multiple languages, including English, French and Spanish (Naber, 2003). The initial version of LanguageTool was written in Python and released in 2003. LanguageTool was, as CoGrOO, integrated to OpenOffice.org in 2006.

LanguageTool performs both style and grammar checking. According to Naber, a style error could be a typographical error, the use of foreign words, or overly lengthy sentences, the misuse of contractions, and others. The same as CoGrOO, it performs shallow processing followed by a rule-based checking. It includes a POS tagger derived from Brill (1992) and a simple Chunker. The chunking strategy is used to find the longest pattern of collocations that match a list of known noun and verb phrases.

To evaluate LanguageTool, Naber used the British National Corpus (BNC, 2003) as a sample of revised text to minimize the number of false errors. He also created two other corpora. The first one was extracted from mailing lists and the errors were annotated and categorized manually. The second one was created by performing Internet searches of error patterns. The results were compiled and used to find cases where the error pattern would not apply, causing a false error.

In its latest version, released in 2012, LanguageTool introduced support for European Portuguese. Currently it is entirely rule based, without morphological analysis.

\subsubsection{LightProof}

LightProof (Nemeth, 2011b) is another Open Source grammar checker currently under development. It is designed to be, as the name advertises, light in the sense that it does not perform language processing like contextual POS tagging and chunking. It is rule-based system supported by the morphological analyzer Hunspell (Nemeth, 2011a). The Portuguese rules for LightProof are currently under development. 


\section{Chapter 3}

\section{CoGrOO - an Open Source Grammar Checker}

The Grammar Checker CoGrOO is a hybrid NLP system, in the sense that it employs statistical analysis to perform text segmentation and categorization, and uses hand-written rules to find error patterns. Up to version 3.x, CoGrOO does not comply with the Portuguese Language Orthographic Agreement of 1990 (OA) (Chiarelli et al., 1990).

This section is an overview of the current version of $\mathrm{CoGrOO}^{1}$, known as 3.1.2. Most of the modules were constructed on top of Apache OpenNLP.

\subsection{Architecture}

Here we will detail CoGrOO's components and architecture. This section is based on papers I co-wrote for ISDA 2007 (Kinoshita et al., 2007) and for Propor 2010 (Silva et al., 2010).

CoGrOO system is composed of the following modules organized as a pipeline:

1. Sentence Boundary Detector: receives a text as input and divides it into sentences;

2. Tokenizer: receives a sentence and divides it into words and punctuation marks;

3. Name Finder: receives the sentence tokens and identifies the potential proper nouns, such as person, places and organization names;

4. Post Tokenizer: receives the sentence tokens and location of proper nouns. It merges composed proper nouns as only one token, and expands contractions. This step is necessary because the training was constructed this way;

5. Part-of-Speech Tagger: receives a sentence and assigns the most probable morphological tag (see tagset in Appendix A) to its lexical items, according to their context;

6. Chunker: receives a tagged text and finds its small noun phrases (NP) and small verbal phrases (VP);

\footnotetext{
${ }^{1}$ I was the first developer to join the project in 2004.
} 
7. Subject-Verb Finder: receives a tagged sentence with NPs and VPs and searches for the subject. If it is found, it marks the NP as a subject of a VP;

8. Grammar Error Detector: this module looks for grammar errors in the input sentence. It is activated after all the previous sentence analysis steps have been done.

Figure 3.1 shows a general view of the grammar-check pipeline.

Since version 2.0, all but Post Tokenizer and Grammar Error Detector modules were constructed on top of OpenNLP (Section 2.2.5), in order to create a more consistent and robust software.

The NLP tools in OpenNLP were created for English, which were initially adapted for Portuguese. This involved changing the MaxEnt features (Ratnaparkhi, 1996) in several internal modules and generating new models using the Brazilian Portuguese resources.

Each of the modules are detailed in the following items.

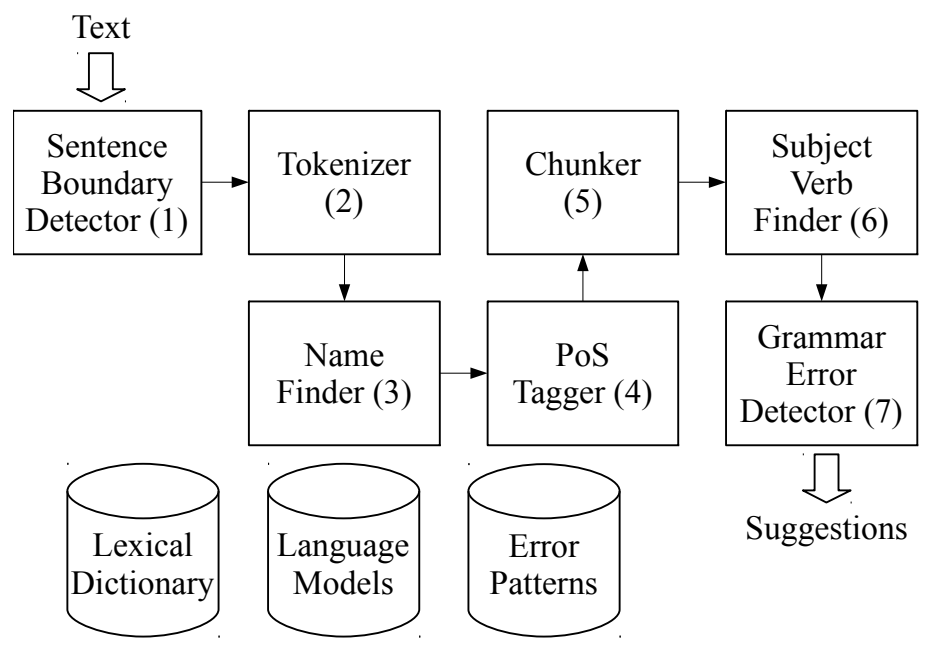

Figure 3.1: Grammar checker pipeline.

\subsubsection{Sentence Boundary Detector}

The sentence boundary detector is responsible for identifying the limit of sentences based on punctuation marks. It checks, for example, if a period mark ends a sentence or if it is part of an abbreviation. A similar operation must be done to other marks that can divide Portuguese sentences, such as exclamation, question and quotation marks, semicolon, brackets, dashes and ellipsis.

Period, exclamation, question, semicolon and ellipsis are considered absolute sentence splitters. Brackets, dashes and quotation marks are only considered if the sentence has a finite verb. For example: o menino disse: "eu quero" e depois arrancou o brinquedo do irmãozinho. (the boy said: "I want" and then, took the toy from his little brother). In this case, the quotation marks highlights a sentence and is a clue that it contains a finite verb.

The steps to detect sentences are: 
1. Identify punctuation marks in the text;

2. Perform a match with some masks to check for constructions that use punctuation marks, but do not define sentences, like e-mail and Internet addresses. These patterns are skipped;

3. Extract features, like the boundaries of the punctuation mark, if the next word starts with capitalized letter;

4. If it is a period mark, add features if it is part of a known abbreviation;

5. Brackets, dashes and quotation mark are considered sentence splitters only if there is at least one finite verb. Add features for that.

Training is done by inputting sentence examples to the Sentence Detector module, which extracts features from it and assembles a database that will be used in the maximum entropy algorithm. Example 3.1 shows how the Sentence Boundary Detector module works on the input text ${ }^{2}$ :

input: O sr. Mendonça chegou. Entrego-lhe os documentos. output: [O sr. Mendonça chegou.] [Entrego-lhe os documentos.]

(Mr. Mendonça arrived. I will give him the documents?).

\subsubsection{Tokenizer}

Sentences are composed of blocks of text, named tokens. The tokens usually represent words, punctuation marks and other symbols.

Generally, sequences of tokens are delimited by spaces. However, there are also several non-trivial issues; for instance if there is no space between tokens, mainly when a punctuation mark follows a word. The tokenizer must detect the tokens.

The CoGrOO tokenizer was constructed on top of the OpenNLP Tokenizer module. The steps are as follows:

1. It divides the sentence into white spaces. Example 3.2:

Entrego-lhe os documentos? $\Rightarrow$ [Entrego-lhe] [os] [documentos?]

(Should I give him the documents?).

2. for each string, it checks its features and compares it with the training data. Some features are:

- The end of the string is a potential token splitter (not alphanumeric)?

- The end of the string is a period mark and it is not part of a known abbreviation?

For example documentos?.

\footnotetext{
${ }^{2}$ Chunks are delimited by brackets.
} 
- The string matches some known construction, like an e-mail address?

- The character after the punctuation mark is a capitalized letter?

The training is done with tokenized sample sentences from the annotated corpus. Through them it is possible to extract features and the maximum entropy algorithm can use it to weigh whether a punctuation mark will be a token or not.

Example 3.3 shows how the Tokenizer module works on the input text:

input: O sr. Mendonça chegou. | Entrego-lhe os documentos?

output: [O] [sr.] [Mendonça] [Chegou] [.] | [Entrego]

\section{[-1he] [os ] [documentos ] [?]}

(Mr. Mendonça arrived. I will give him the documents.).

\section{Name Finder}

Proper nouns in the Brazilian Portuguese corpus (CETENFolha) are arranged in a single token. For example, the name "Jane Doe" is represented as the token "Jane_Doe". Due to this, to use this corpus as training data for the tagger, it should first find the proper nouns of the sentence and group them.

CoGrOO uses the Name Finder module of OpenNLP. The training process is done by sending to the module sentences with the proper nouns marked with tags:

$$
\text { I think <START>Jane Doe<END> is coming. }
$$

and the module will search for the characteristics of the occurrence and then evaluate the importance of each characteristic to determine if it should be considered when evaluating new texts. These features are:

- Capitalization;

- Tokens classified as proper nouns in the dictionary;

- Unknown word (not in the dictionary);

- Occurrence of prepositions between nouns.

Example 3.5 shows how the Name Finder module works on the input text:

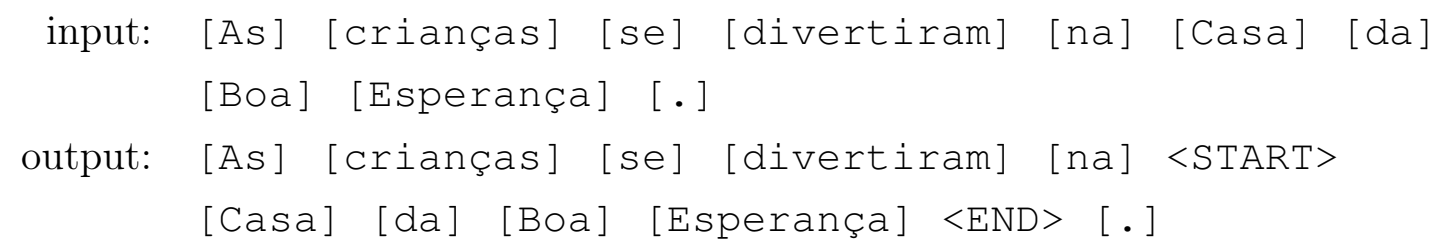


(The children had fun in the House of Hope.).

\subsubsection{Post Tokenizer}

The Post Tokenizer is responsible for rearranging tokens. 1) it will take the proper names from the Name Finder module and merge its tokens in only one token; 2) it will expand contracted forms. The OpenNLP framework does not include a ready-to-use module to do this kind of processing, so the Post Tokenizer module was developed from scratch.

This post tokenizer processing is necessary because the Brazilian Portuguese corpus (CETENFolha) tokenizes texts this way: all composed proper names are grouped together as one token and all the contractions are expanded.

According to Silva (2007), contractions are strings that, syntactically, correspond to a sequence of words, even though they are written as a single token. In Portuguese orthography, most of these strings correspond to the contraction of a Preposition - usually de or em with the Article or Pronoun that follows it: da (de + a), nele (em + ele), consigo $(\mathrm{com}+\mathrm{si})$, etc.

The current version of CoGrOO performs post tokenization as following:

1. Replaces tokens that are part of a proper name by the first token of this proper name sequence;

2. Replaces the contracted forms by the equivalent expanded form.

Currently CoGrOO does not check for ambiguous strings, like the word consigo, which, according to the context, can be the contracted form of com $+\mathrm{si}$, or a conjugated form of the verb conseguir.

Example 3.6 shows how the Name Finder module works on the input text:

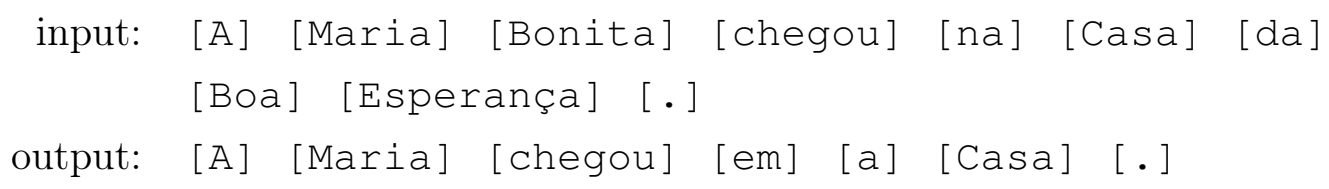

(Maria Bonita arrived at the House of Hope.).

\subsubsection{Part-of-Speech Tagger}

The POS Tagger module assigns morphological and inflection tags to tokens. CoGrOO uses the POS Tagger module of OpenNLP, with a few additions to the default features:

- Better support for grouped proper names;

- Features associating capitalized letters with proper names;

- Features related to the dictionary, for example, whether there is a word or not; 
- Features to improve performance of hyphenated words.

The tag set adopted is a subset of the Constraint Grammar category set ${ }^{3}$ of Palavras, and includes word class tags and inflection tags. See Appendix A for tag reference.

Example 3.7 shows an output example:

\begin{tabular}{l|l|l} 
Token & Lemma & POS Tag \\
\hline A & O & determiner, female, singular \\
Maria & Maria & proper noun, female, singular \\
chegou & chegar & finite verb, 3rd person singular, past perfect, indicative \\
em & em & preposition \\
a & $\circ$ & determiner, female, singular \\
Casa & Casa & proper noun, female, singular \\
• & $\cdot$ & punctuation mark, abs
\end{tabular}

\section{Multi-Word Expressions}

The corpora used to train the POS Tagger contains Multi-Word Expressions (MWE), which consist of a lexeme composed of two or more lexemes that together express functions and semantics that can not be predicted from their individual parts. The following example was extracted from the training corpora:

\begin{tabular}{l|l|l} 
Token & Lemma & POS Tag \\
\hline Muito & muito & adverb \\
mais & mais & adverb \\
do_que & do_que & conjunction \\
em & em & preposition \\
os & os & determiner, male, plural \\
tempos & tempos & proper noun, male, plural
\end{tabular}

(Much more than in the time).

\begin{tabular}{l|l|l} 
Token & Lemma & POS Tag \\
\hline Com_base_em & com_base_em & preposition \\
seu & seu & pronoun \\
estatuto & estatuto & noun, male, singular
\end{tabular}

(Based on their statute).

The current implementation of CoGrOO does not handle the MWE from the corpora and always tag the lexemes of a MWE individually.

\footnotetext{
${ }^{3}$ Constraint Grammar category set of Palavras, http://beta.visl.sdu.dk/visl/pt/info/portsymbol.html, lastaccessedon $12 / 10 / 2012$
} 


\subsubsection{Chunker}

Text chunking consists of dividing a text into syntactically correlated groups of words, like noun groups and verb groups, but does not specify their internal structure or their role in the main sentence.

The corpus CETENFolha Cortado (Section 2.3.2), used to train CoGrOO until version 3.1, does not include any structure information, so it was not possible to train the OpenNLP Chunker directly.

CoGrOO uses a list of patterns that were developed to recognize noun and verbal phrases. The following is an example of pattern (3.10):

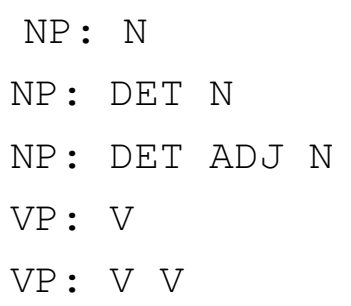

The training strategy is used to recognize phrases in the corpus, and use this information to train the OpenNLP Chunker. The maximum entropy algorithm can learn from the matched patterns and perform better (Kinoshita et al., 2007).

The chunker module takes the sentence with POS tags as input. Example 3.11 shows what the output looks like:

$$
\text { [NP A Maria] [VP chegou] em [NP a Casa]. }
$$

\subsubsection{Shallow Parser}

The purpose of this module is to detect the subject (a noun phrase) and a verb (a verbal phrase) relationship. The output of the chunker and the tagger is submitted to this module. It searches for a NP-VP pattern, i.e., a pattern of tags, NPs and VPs. Example 3.12 is an example pattern. The mark (!) denotes the beginning of the sentence.

$$
\text { ! NP VP }
$$

If there is a noun phrase in the beginning of a sentence followed by a verbal phrase, then the pattern is matched and the noun phrase will be assigned as the subject of the verbal phrase.

These patterns were automatically extracted from CETENFolha corpus. Whenever the syntactic tag SUBJ, indicating subject, appears in the corpus, an NP-VP pattern is extracted.

CoGrOO uses an adapted OpenNLP Chunker for the shallow parsing task. To train using the maximum entropy algorithm, the noun phrases that are part of the subjects are grouped 
as a subject chunk. The same is done for verb phrases.

Example (3.13) is a possible output of the shallow parser module:

[SUBJ A Maria] [V chegou] em a Casa.

\subsubsection{Grammar Error Detector}

This module detects possible errors in the input sentence through the use of hand-written error rules. An error rule is just a pattern that is searched in the input sentence. These rules are based on common grammar errors in Brazilian Portuguese.

A typical example of error detection by this module is the use of accent stress marker which is the contraction between the preposition a (to) and the singular feminine definite article a (the) - before masculine words or verbs. So, an example of an error rule is:

$$
\text { "a", "a", verb }
$$

This rule represents a à (a with an accent stress marker) followed by a verb. In this case, à is represented by the sequence ("a", "a") because in Portuguese à is the contracted form of "a" + "a"), and CoGrOO uses the latter representation.

The rule patterns are compiled into a Finite State Machine and applied to the text. Each rule is composed of:

- Pattern: a sequence of features that is applied to a token. Available features are: lexeme, lemma, POS tag, chunk tag, syntactic function (subject or verb), or a combination of features.

- Method: can be local, delimited by phrases, or structural;

- A short message, which can be displayed in a context menu for example;

- A long message, with more details about the error;

- Information about which portion of the matched text should be marked as wrong;

- Information about how to generate a replacement suggestion;

- Examples of sentences with the error, and the equivalent correct sentence.

The module input is the sentence with data provided by the former modules: sequence of tokens, tags, phrases and subject-verb markers. The output is a data structure which marks the error in the sentence. It is composed of an error message, the wrong sentence and suggestions on how to deal with the grammar error.

The Grammar Error Detector module is activated at three different moments: after POStagging, after chunking and after shallow parsing. After POS-tagging, we have the local rules that are applied to a short sequence of words and tags. For the treatment of more complex errors like verb agreement errors, we apply structural error rules in the output of Chunker and Subject-Verb Finder modules. 
Aiming to observe how the other modules affect the effectiveness of the Grammar Error Detector, this module is not going to be changed in this work.

\subsection{Annotator Accuracy}

As CoGrOO is distributed as an OpenOffice.org extension, an important nonfunctional requirement is its package size, which should be as small as possible. On the other hand, the size of the package is related to the dictionaries and model sizes. Reducing them can decrease performance.

To achieve a good balance between package size and annotation performance, CoGrOO development tools include training mechanisms which allow fine tuning of the number of sentences in the training set. This is controlled by a cutoff parameter (minimum number of times an event should occur before it is included in the model) and the number of training samples. If the cutoff grows too large or too small, the performance decreases; if the cutoff increases, the package size decreases. The model performance increases with the number of samples, but on the other hand the package size also increases. So knowing the optimal cutoff and number of samples is required.

The annotators were evaluated using 10-fold Cross Validation (Section 2.1.3) using the CETENFolha Cortado, described in Section 2.3.2. The performance of annotators are shown in Table 3.1.

\begin{tabular}{|c||c|c|c|c|c|c|}
\hline Module & Accuracy & Precision & Recall & F1 & Sentences & Cutoff \\
\hline \hline Tokenizer & - & 0.954 & 0.975 & 0.964 & 40000 & 150 \\
\hline Name Finder & - & 0.941 & 0.946 & 0.943 & 450000 & 250 \\
\hline POS Tagger & 0.961 & - & - & - & 50000 & 5 \\
\hline Chunker & 0.772 & - & - & - & 20000 & 150 \\
\hline Shallow Parser & 0.688 & - & - & - & 20000 & 100 \\
\hline
\end{tabular}

Table 3.1: Effectiveness of CoGrOO 3.0 annotators. For Chunker the precision was computed considering sentences with all chunks correctly annotated. For the Shallow Parser, the precision was computed considering sentences with all subjects and main verbs correctly annotated.

\subsubsection{Discussion}

The annotator accuracy does not reflect the grammar checker effectiveness. There are a number of issues related to training and evaluation that impacts these figures.

Sentence Detector and Tokenizer In the corpus used to train CoGrOO 3, the CETENFolha Cortado, described in Section 2.3.2, numbers were replaced by placeholders. Due to this, the sentence detector and tokenizer modules do not properly handle numbers with decimal or group separators. These have a significant impact on the grammar checker, but do not affect the module accuracy while performing cross-validation evaluation. 
POS Tagger CoGrOO 3 uses a lexical dictionary that was constructed from a number of sources, including the corpus itself. Due to this, the POS Tagger dictionary knows all the possible POS tags for each word of the evaluation set. This should explain the high accuracy of the POS Tagger module while performing 10-fold cross-validation in the same corpus.

Chunker and Shallow Parser The CETENFolha Cortado, described in Section 2.3.2, does not include sentence structure information. Alternatively, a set of rules, as described in Section 3.1.5, were used to both train and evaluate these modules. Also, the original evaluation tools could not measure the effectiveness in terms of $F_{1}$, making it harder to compare their scores to other systems.

\subsection{CoGrOO UIMA}

The OASIS Standard platform UIMA ${ }^{4}$ offers a reference architecture for NLP systems and the Apache UIMA ${ }^{5}$ a framework to help its realization. Internally, CoGrOO does not use UIMA, but its annotators were also bundled as standard UIMA Annotators and made available as open source software and now can be used by any UIMA based application (Silva et al., 2010) ${ }^{6}$.

The Apache UIMA implementation allows each CoGrOO UIMA annotator to run in a different Java classpath, allowing mixing annotators that depends on different versions of the library. This characteristic will facilitate evaluating the improvements: it will allow trying a new version of an annotator keeping all other annotators unchanged and evaluate how it impacted the overall results. More details on how UIMA were used during the evaluation can be found in Section 4.4.

\subsection{CoGrOO Community}

CoGrOO Community ${ }^{7}$ is a web application that provides collaborative tools for grammarcheck users. Some features are: a) an online grammar checker where users can view the outputs of low-level modules (i.e. POS tags and chunks); b) browse the lexical dictionary; $c$ ) browse the rule database; $d$ ) browse and include problem reports. The most relevant feature for this work is the problem report.

The problem report page allows users to submit sentences that caused CoGrOO to fail, indicating whether it is a false negative or false positive. In each case the user provides

\footnotetext{
${ }^{4}$ The UIMA specification is being developed concurrently by a technical committee within OASIS: http: //www.oasis-open.org/committees/uima, last accessed on 07/30/2012

${ }^{5}$ Apache UIMA is a open source implementation of OASIS Standard platform UIMA: http://uima.apache. org, last accessed on $07 / 30 / 2012$

${ }^{6} \mathrm{CoGrOO}$ UIMA Annotators are available at: http://ccsl.ime.usp.br/cogroo/uima, last accessed on $10 / 18 / 2012$

${ }^{7}$ Cogroo Community site: http://comunidade.cogroo.org, last accessed on 12/10/2012
} 
relevant classification details. This error list will be used to evaluate the types of errors that beset users the most, and will be part of a new corpus that also could be used to evaluate CoGrOO improvements. The corpus derived from the reported problems includes 378 sentences, which 104 are flagged as grammatically incorrect. 


\section{Chapter 4}

\section{A new grammar checker proposal}

The initial evaluation, which will be detailed in Section 5, highlights the main weakness of CoGrOO: an alarming number of false positives it provides. The new grammar checker proposal focuses on reducing this number by improving its underlying modules. Each intervention is measured by experiments that compare the impact of the changes.

Upgrade its dependencies: Updates for many of the underlying libraries are available, but often their APIs are not backward compatible, including Apache OpenNLP, of which even the models created for CoGrOO 3.1.2 are not compatible with the latest version of the library. To allow for bug corrections and to take advantage of the new features provided by these libraries, CoGrOO should migrate them to the latest versions.

Introduce JSpell.br dictionary: The JSpell.br dictionary (Section 2.3.1) will be the official dictionary. The current dictionary is derived mainly from the training data and proved to be an important source of issues.

Introduction of new modules: Training new Apache OpenNLP modules for Portuguese, fine-tuning wherever possible with language specific features and additional resources.

A new pipeline: The new pipeline should be compatible with the latest Apache OpenNLP code and as well be customizable to allow easy introduction of new modules.

Figure 4.1 shows a general view of the new grammar-check pipeline. Some of the improvements were introduced directly in Apache OpenNLP code, as explained in the next section. Refer to Section 4.2 for an overview of the system architecture.

\subsection{Apache OpenNLP 1.5.x}

The Apache OpenNLP framework, introduced in Section 2.2.5, is the core of the CoGrOO grammar checker because many of its algorithms were constructed on top of that framework. 


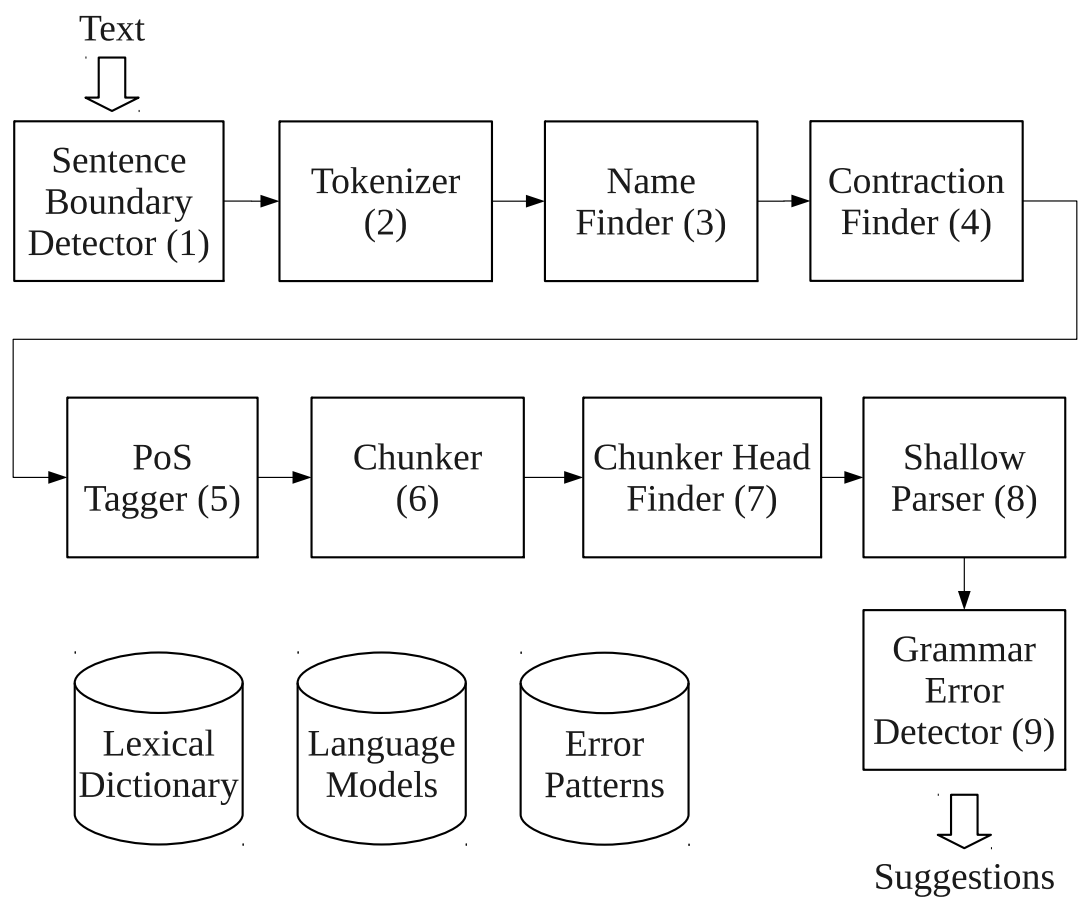

Figure 4.1: General view of the new grammar-check pipeline.

The current version of Cogroo relies on Apache OpenNLP 1.4.3, while the most recent version of this library is 1.5.2.

The plan is to continue using Apache OpenNLP, but also to update to its latest version, which requires a great deal of code change in the grammar checker due to a compatibility break between the Apache OpenNLP versions.

Also, as Apache OpenNLP is a popular framework used by many other projects, it was important to, as far as possible, add new functionalities to the framework instead of to CoGrOO, which is dedicated to Portuguese. The functionalities added to OpenNLP are:

- Formatters to read the Arvores Deitadas corpus;

- Extension capabilities, which allow easier customizations of context generators, sequence validators and resources (dictionaries for example);

- The capability to attach customized evaluation listeners and reports;

- A listener that outputs details for each error;

- A fine grained evaluation report for POS Tagger (tokens with the highest number of errors, most frequent tokens, detailed accuracy by pos tag, general confusion matrix and confusion matrix for each token);

- A detailed F-Measure report that can be used by any tool that outputs $F_{1}$; the report includes detailed global score and detailed $F_{1}$ score for each output type, which includes target, true positives and false positives count); 
and many others. The complete list of changes in Apache OpenNLP related to Cogroo project can be found in the project issue tracker ${ }^{1}$.

\subsection{The New Software Architecture}

The new API of CoGrOO is based on the pipeline pattern, as previously shown in Figure 4.1. Each processing element of this pipeline extends the Analyzer interface and is responsible for a natural language processing task. The class diagram from Figure 4.2 shows some API classes, where only three analyzers are visible for simplicity. Analyzers can bundle an OpenNLP annotator, for example a Tokenizer, or bundle a grammar-check specific task, for example the Grammar Checker Analyzer. Each analyzer can add specific annotation to a document, which is represented by the diagram class from Figure 4.3.

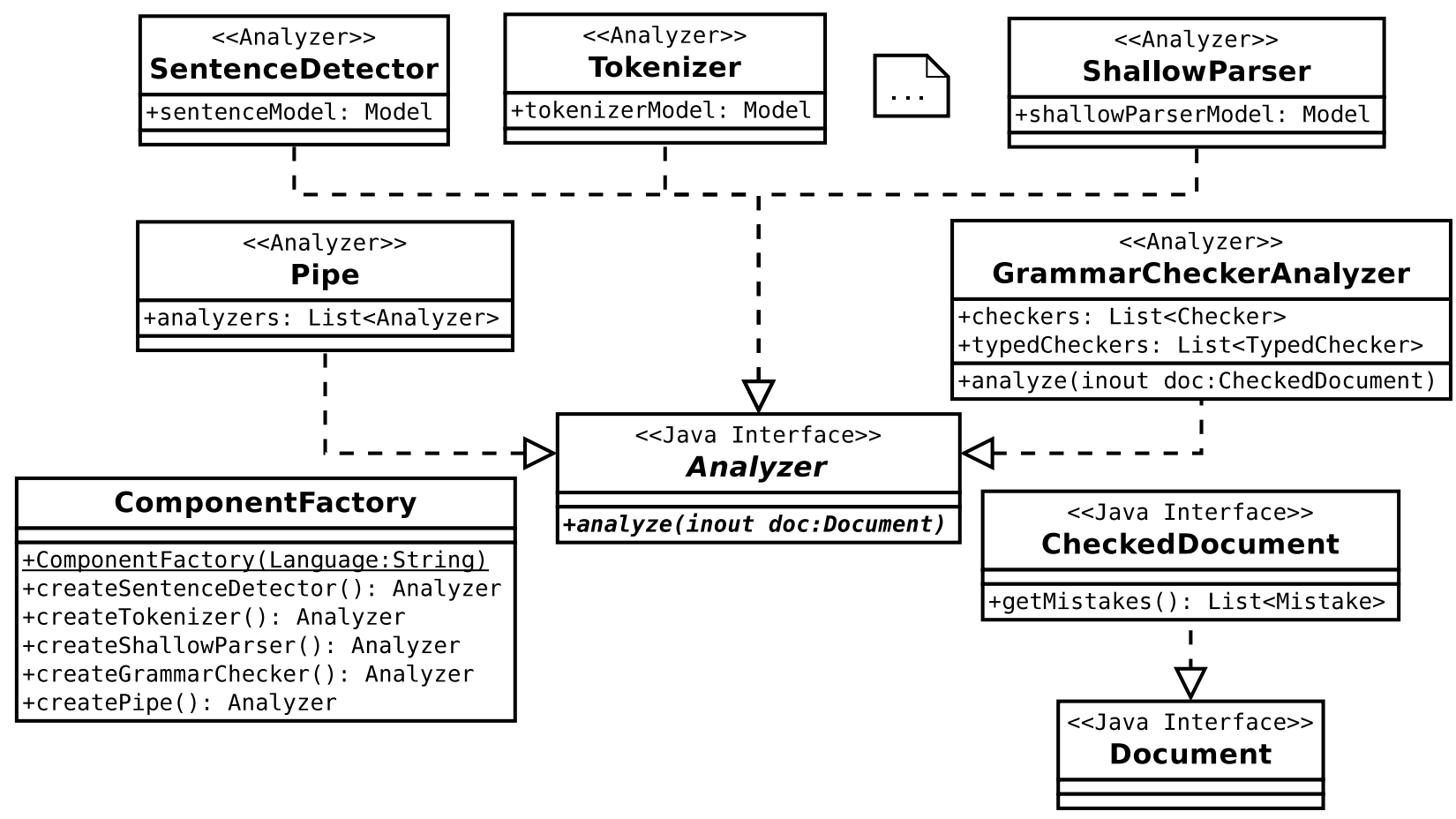

Figure 4.2: Class diagram of the Pipe and Analyzers API

ComponentFactory is responsible for creating the analyzers and the Pipeline. It is configurable, which allows easily trying a different grammar checker's configurations, as well as multi-languages. To get an instance of ComponentFactory, one can pass in a model configuration file, whose Brazilian Portuguese version is in Code 1. For model based analyzers, such as the OpenNLP based, the configuration includes a section where it is possible to bind the analyzer to a model in the classpath. The pipeline section describes the order in which each analyzer is added to the pipeline. Finally, the location binds the configuration to a language, allowing users to create a ComponentFactory object using a location description instead of a path to the configuration file.

\footnotetext{
${ }^{1}$ Apache OpenNLP issue tracker, https://issues.apache.org/jira/secure/IssueNavigator.jspa?reset= true\&jqlQuery $=$ project $+\% 3 \mathrm{D}+\mathrm{OPENNLP}+\mathrm{AND}+$ assignee $+\% 3 \mathrm{D}+$ colen, last accessed on 12/10/2012
} 


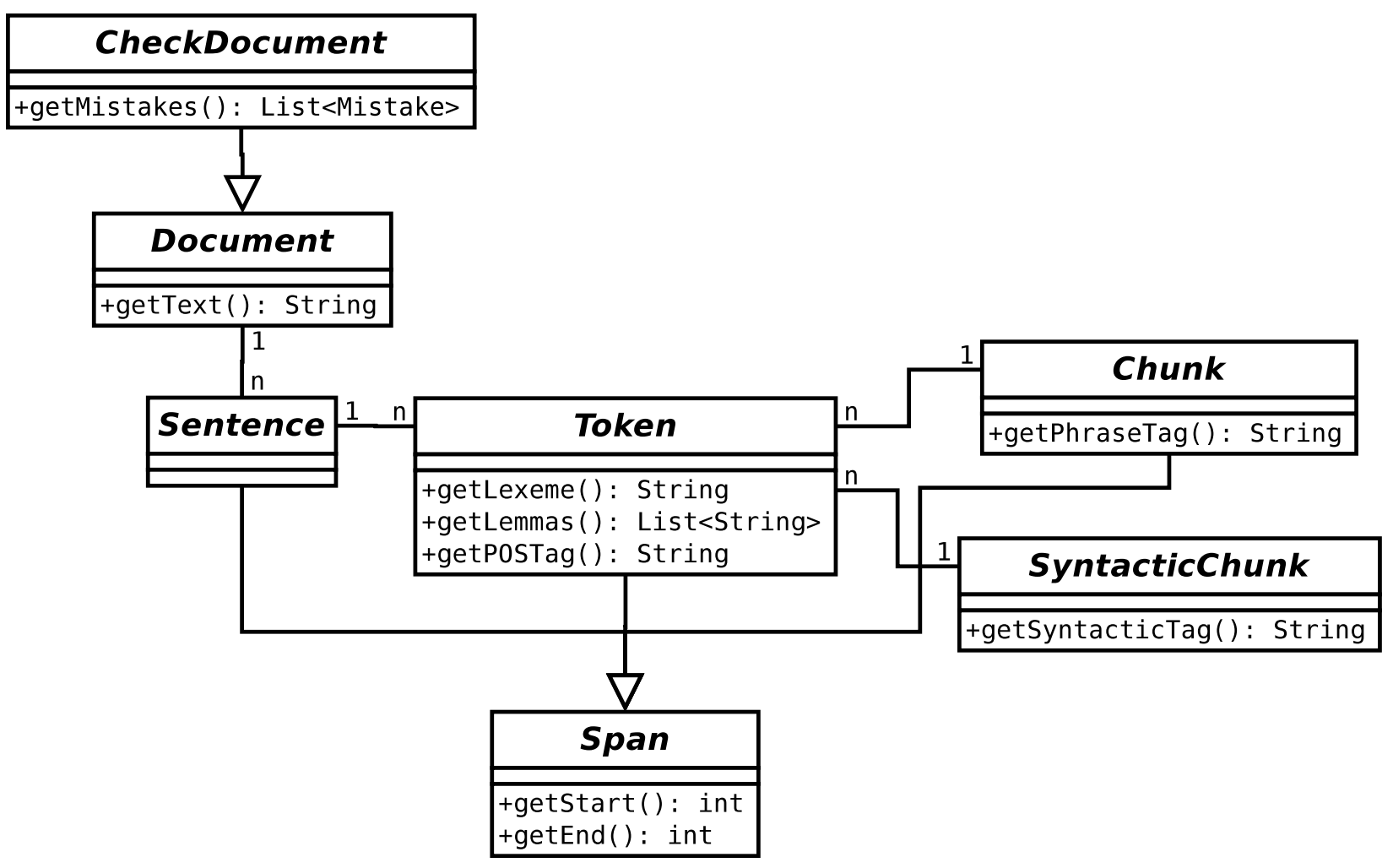

Figure 4.3: Class diagram representing Document and related data structures.

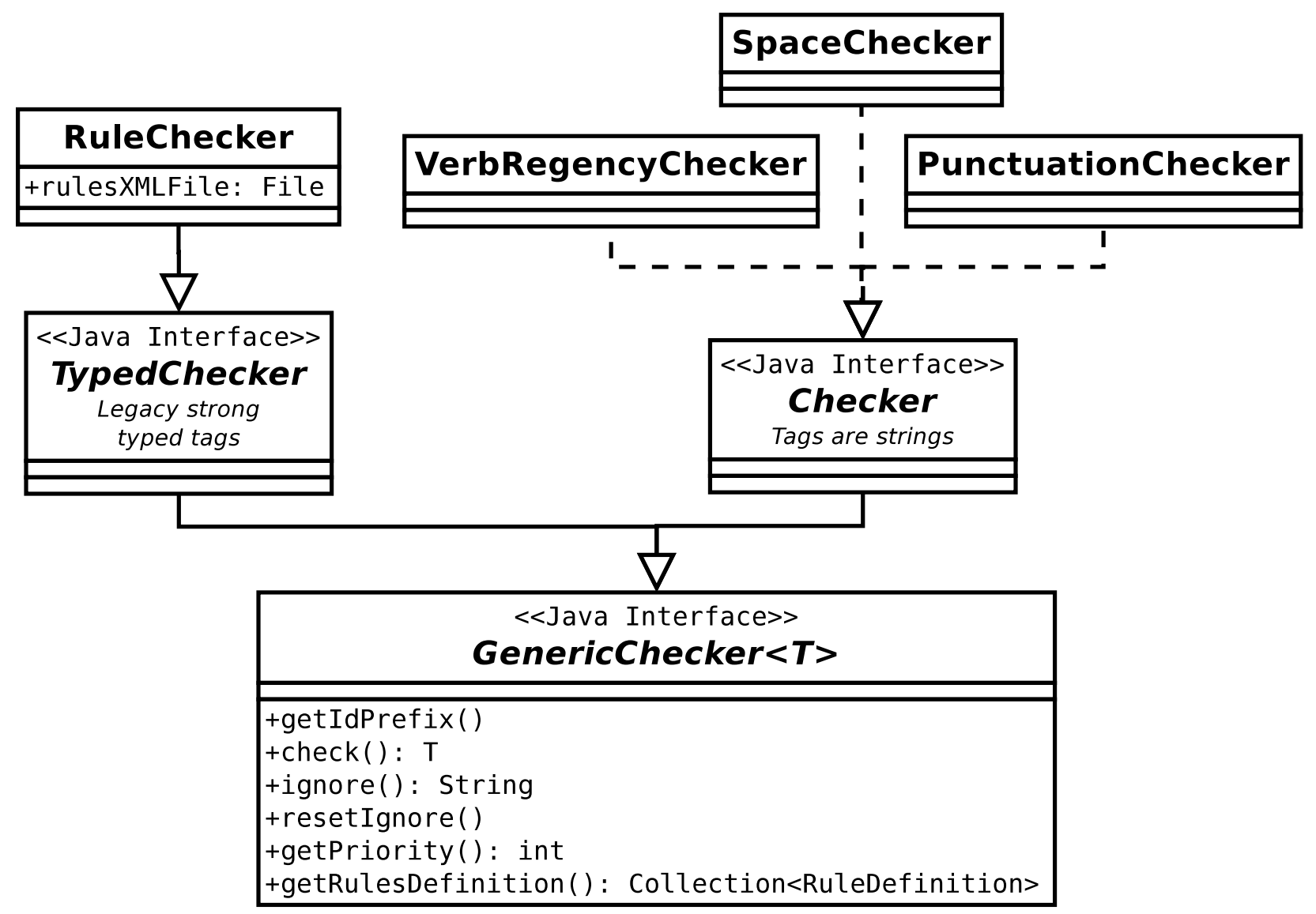

Figure 4.4: Checker class diagram 


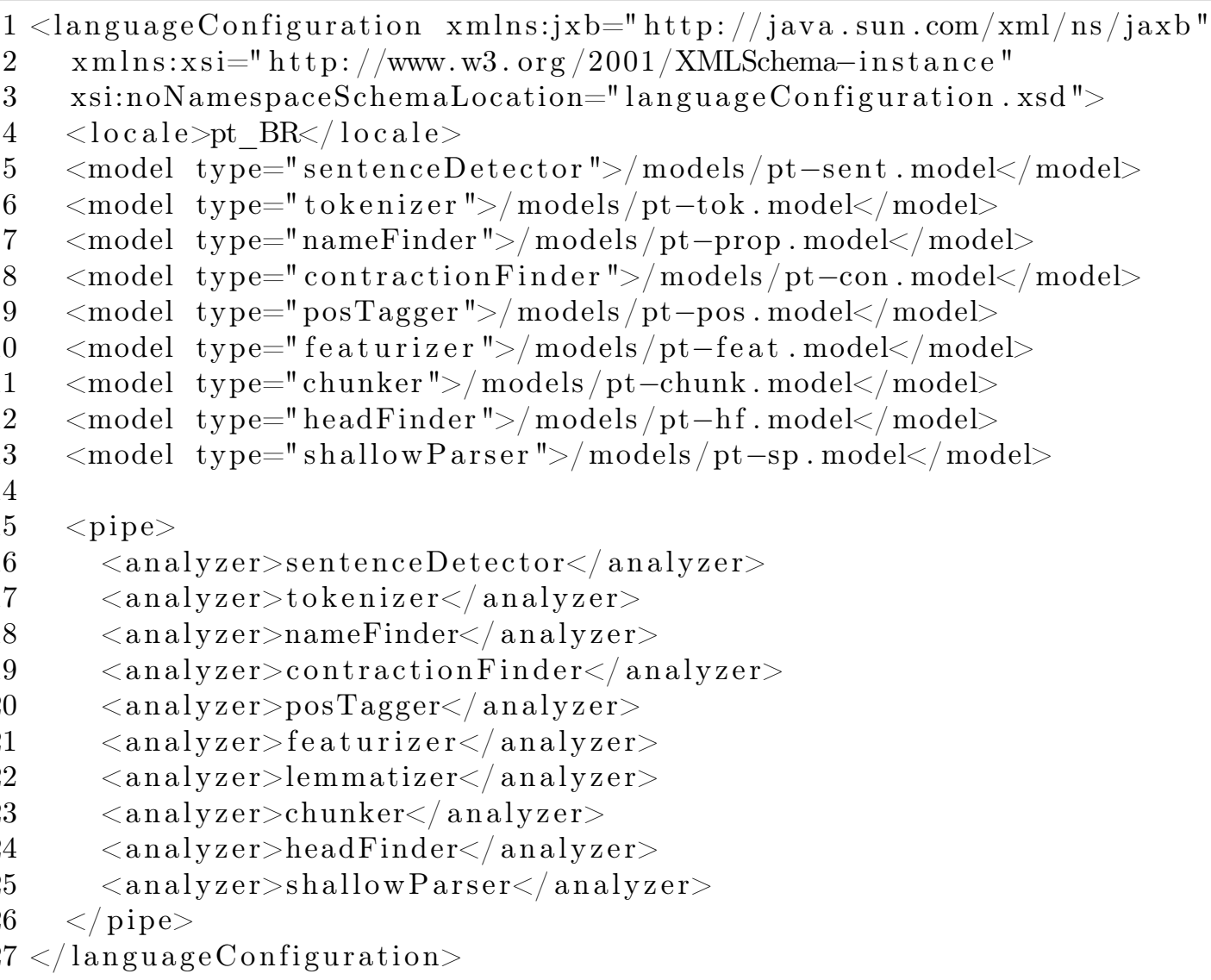

Code 1: Models configuration for Brazilian Portuguese.

A checker, Figure 4.4, is a special Analyzer that uses the Document structure to create a checked document. It is possible to plug in as many checkers as it is convenient, but in the experimental phase only the RuleChecker was used because it was the only checker available for CoGrOO 3.1.2 and adding other checkers would increase the number of rules, which was not the aim of this work.

The sequence diagram from figure 4.5 shows how the grammar checker is initialized and the process of analyzing a document. An user initializes the ComponentFactory passing the desired language. The ComponentFactory loads the configuration file and initializes the pipeline. After, the user sends a document to the pipeline. The box in the top right corner details how each component is initialized.

A persistent OpenNLP model is self contained in the sense that during runtime the users do not need to pass on any configuration to the component. Ideally, any resource required by the model, for example a dictionary, should be bundled inside the model. A Factory class is declared by the model and used to load the right resources and classes. The class diagram from Figure 4.6 illustrates this for Sentence Detector. To customize the component one should extend the default factory, overriding the resources, context validator and generator getters, which can be as well extended. 


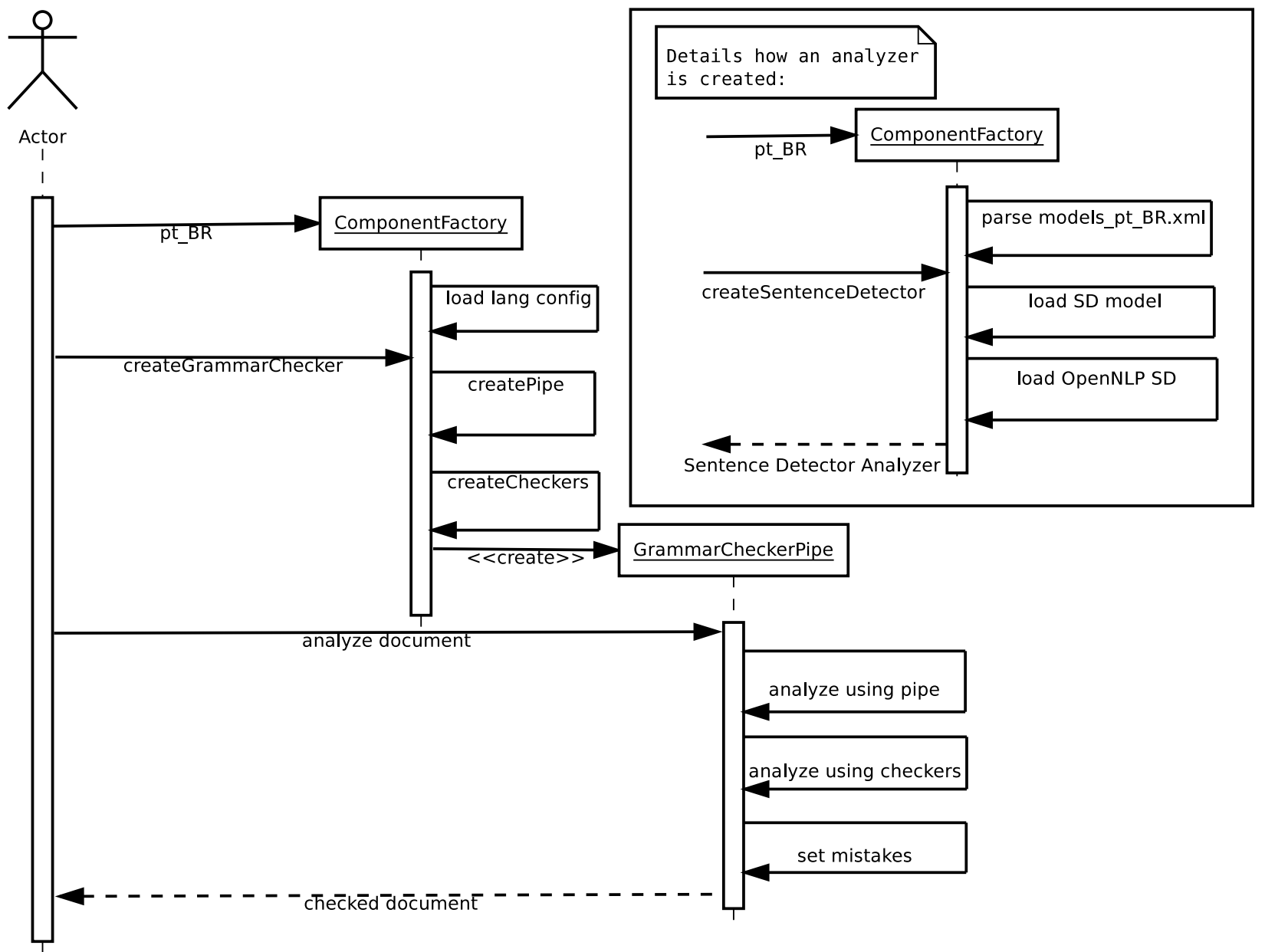

Figure 4.5: Sequence diagram describing the startup and runtime processes.
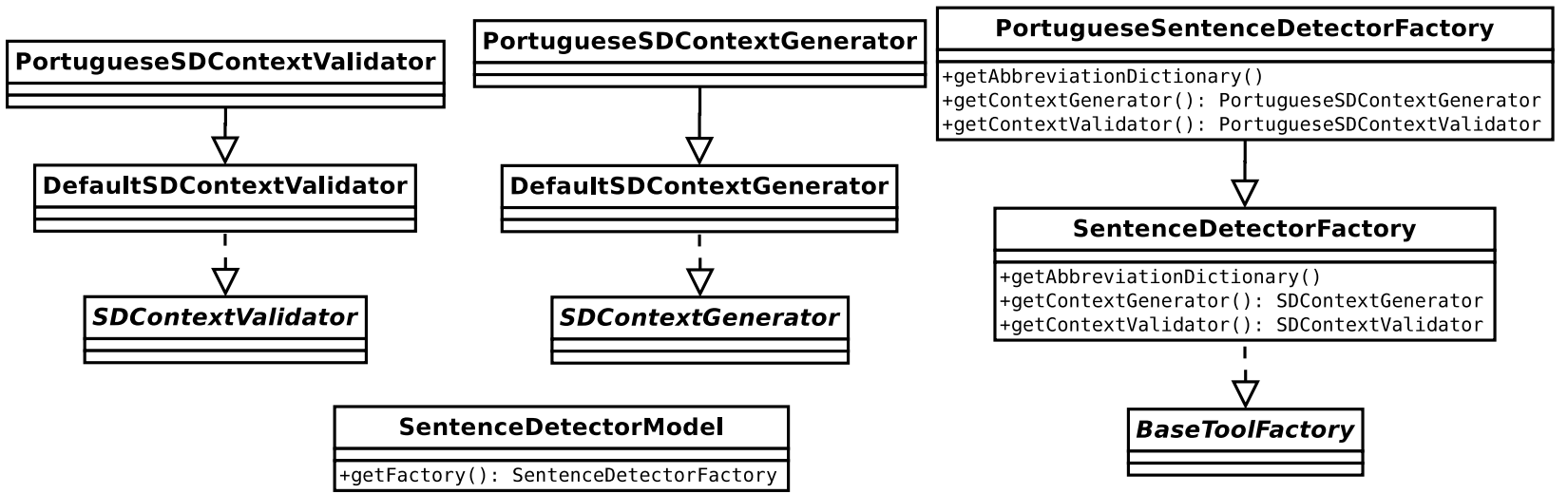

Figure 4.6: Customizing OpenNLP model by extending the tool factory.

\subsection{Experiments Plan}

The objective of the experimental phase of this project is to spot bottlenecks of the grammar checker processing pipe, especially those causing false positives. The experiments are divided into three phases: 
Initial evaluation In this phase CoGrOO 3.1.2 is tested for true positives and false positives using the evaluation corpora. This experiment is covered in Chapter 5.

NLP Development and Evaluation During this phase, each of the CoGrOO proposed modules are individually developed and tested. The development consists of the creation of a new implementation module, followed by an evaluation of each different implementation using a 10-fold cross-validation. Finally, each implementation is evaluated by measuring its impact in the grammar checker effectiveness. After this process, the best module is added to the new grammar checker baseline, which is used in the development of the following modules. This is covered in Chapter 6 .

Final evaluation The final evaluation measures how the new modules impacted the grammar checker effectiveness and compares the results with the initial evaluation, as well as with other grammar checkers. These experiments are covered in Chapter 7.

\subsection{Experiment Preparation}

A set of tools are required during the experimental phase: copora readers, 10-fold crossvalidation tools and grammar checker evaluation tools. The following sections detail these tools.

\subsubsection{Corpora preparation}

As described in Section 2.1.2, corpora are required in two development steps: as training and evaluating data in the development of statistical modules, as well as to evaluate the grammar checker. The available corpora for both tasks were listed in Section 2.3.2.

For training and evaluating the statistical modules three corpora were effectively used. Bosque was used for modules that classify tokens, like a POS tagger. These modules require higher precision instead of quantity of information. Floresta Virgem and Amazônia are better for modules that work at the sentence level, like a tokenizer or sentence detector, which takes advantage of the higher number of tokens of these two corpus. The three are part of the Floreseta Sintá(c)tica project, Section 2.3.2.

Three corpora were used during the grammar checker effectiveness evaluation; two containing grammar error annotations: PROBI (Section 2.3.2) and Metro (Section 2.3.2), and the Bosque (Section 2.3.2) corpus, whose documents were professionally edited but did not include grammar error annotations, was used to measure the number of false positives. CoGrOO Community corpus (Section 3.4), whose sentences were submitted by users of the grammar checker, was also annotated with grammar errors, and is used in the initial and final evaluation of this work, which are respectively described in sections 5 and 7 . 
PROBI and Bosque are available in a computer friendly format, but the Metro, which consists of an HTML document with a list of uncategorized grammar errors and a collection of texts ${ }^{2}$, is not. Metro corpus had to be manually converted to a compatible format.

CoGrOO Community Corpus was manually revised to check whether the sentences classified by the users as false positive or false negative were correctly annotated. For example, some users submitted sentences which errors were actually related to misspelled words instead of grammar issues. From 492 sentences, only 378 were effectively used during the grammar checker evaluation.

\subsubsection{0-Fold Cross-Validation Tool}

A tool was designed to automatically execute predefined sequences of 10-fold crossvalidation experiments. It takes an Apache OpenNLP, for example the sentence detector, plus a set of configurations, which includes:

- The training corpus;

- The machine learning algorithm (Maxent or Perceptron);

- Tool specific configurations, for example, whether to use an abbreviation dictionary or not, or which dictionary to use.

These configurations are used to create and evaluate models varying the cutoff value. For now, the cutoff values are 0, 2, 4, 8, 16, 32, 64, 128, 256 and 512. Each experiment outputs:

- Effectiveness values (accuracy, precision, recall, f-measure);

- Total number of predicates in the model;

- The model size;

- Time required to train and evaluate the model.

These results can be used to find the configuration with the highest effectiveness measure. Several consolidated versions of this report, which allow comparing configurations side-byside, are used in the evaluation phase of the project. The complete reports are available both in the CD provided with this document, as well as online at the project website.

\footnotetext{
${ }^{2}$ The HTML report is available at http://www.pcs.usp.br/ cogroo/papers/analise-cogroo-corpus-metro. html
} 


\subsubsection{Grammar Checker Evaluation Tool}

Some pieces of software were created for the experimental phase. The evaluation software should read a corpus (Corpora Reader) and execute the grammar checker in each of the corpus sentences (Evaluator). According to the corpus annotation regarding grammar mistakes, the evaluator should check each intervention and compute the number of true positives, false positives and false negatives. The values are used to compute the precision, recall and f-measure of the system.

To individually measure the impact of improving each component of the grammar checker, its pipeline should support replaceable components; This should allow, for example, replacing the original sentence detector by a new one and keeping all other original components. A grammar checker pipeline with this capability were created and called the Grammar Checker Adapter. With this adapter each component of the grammar checker can be easily replaced, following a configuration file.

Figure 4.7 provides a schematic view of the evaluation tool.

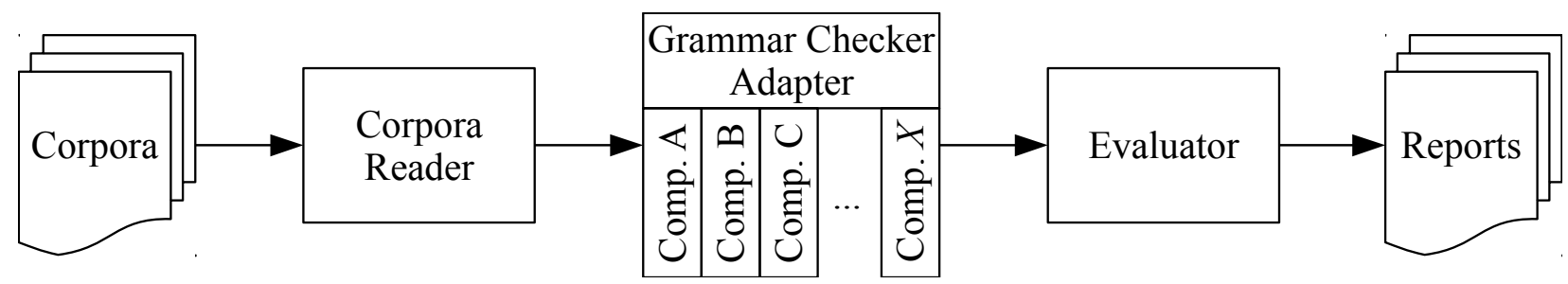

Figure 4.7: Grammar checker evaluation tool.

The evaluation tool was written as UIMA components for several reasons. Firstly, UIMA standardize a clean and well known architecture to design natural language processing tools. Secondly, the PEAR packaging allows a mixed classpath, which means it allows having components that require different versions of the same library executing together, handling any API conflict. This is a requirement because CoGrOO 3 uses older versions of libraries, for example OpenNLP 1.4, and the proposed grammar checker would be upgraded to the latest versions.

The Corpus Reader components were designed as UIMA Collection Reader. Two readers were created, one to parse the Árvores Deitadas and the other to parse PROBI formats.

The Grammar Checker Adapter is an UIMA Annotator that bundles a modified version of the grammar checker. The main modification is that each component of the grammar checker can be replaced according to a configuration file. To allow for mixing different versions of the same component that requires different library versions, each component is bundled as a UIMA Annotator as well, packaged as a PEAR file.

The Evaluator component is an UIMA CAS Consumer that compares the output of the grammar checker with the corpus annotations. It generates two different reports. The first is an HTML file that summarizes the evaluation results with a few tables and charts: a table with the global and individual precision, recall and f-measure results, for each category 
(Appendix B); a pie chart with the distribution of grammar error categories; a column chart illustrating the precision and recall for each grammar error category; a column chart with the count of false positives in the grammar error categories; a pie chart showing the percentage of uncovered grammar error categories; a sequence of Venn charts to illustrate the distribution of target and selected items for each grammar error category as well as for the overall experiment. The second is a text report whose lines contain each corpus sentence with annotations classifying it as true or false positive, or false negative. This report can be used to check the sentences which analysis changed after each modification in the grammar checker.

The UIMA components are aggregated in a set of Collection Processing Engine configurations, one for each corpus. Each combination can be executed using the UIMA CPE tool. Figure 4.8 shows the CPE tool configured to execute an experiment with the Metro corpus.

The reports are used during the grammar checker evaluation, and the relevant results are included in this document as consolidated tables. The complete reports are available online at the project website ${ }^{3}$. Appendix $\mathrm{C}$ provides instructions on how to reproduce the experiments and generate the reports.

\footnotetext{
${ }^{3} \mathrm{CoGrOO}$ Research and Development page: http://cogroo.org/pesquisa_e_desenvolvimento, last accessed on $04 / 26 / 2013$
} 


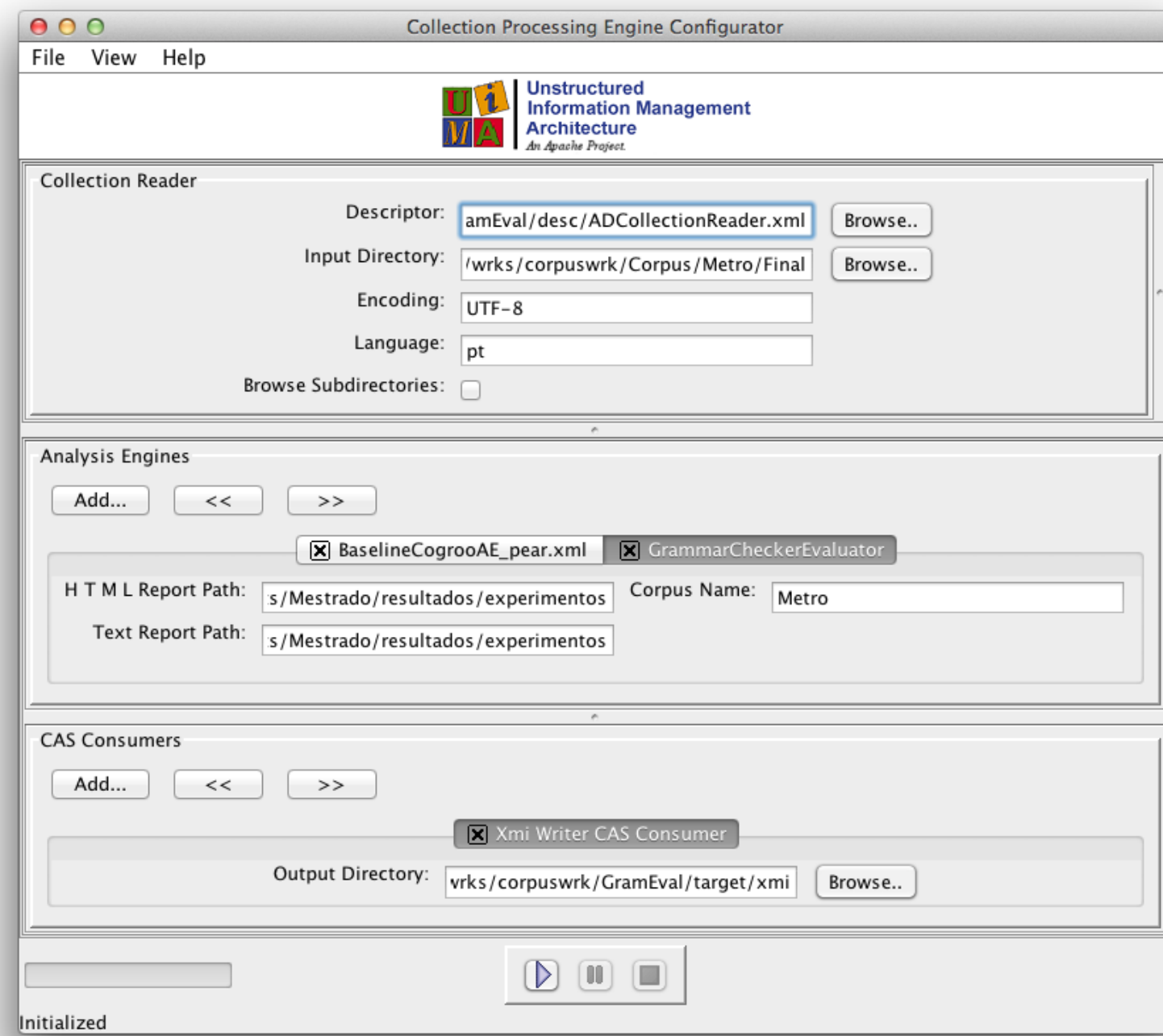

Figure 4.8: Grammar checker pipeline configured with the Collection Processing Engine tool of the UIMA framework. 


\section{Chapter 5}

\section{Initial Evaluation}

This section gathers an initial snapshot of the grammar checker effectiveness. The latest version of the grammar checker, 3.1.2, was used as baseline and will be referred to here as BASELINE.

\subsection{PROBI Corpus}

PROBI Corpus (Section 2.3.2) includes 11,625 sentences, which 2,616 are flagged as grammatically incorrect. The BASELINE selected 1,538 sentences as containing grammar errors, but only 357 of those were correctly selected according to the corpus annotation. Table 5.1 presents a summary of the results.

$\begin{aligned} \text { Sentences: } & 11625 \\ \text { Target: } & 2616 \\ \text { Selected: } & 1538 \\ \text { True positives: } & 357 \\ \text { Precision: } & 23.21 \% \\ \text { Recall: } & 13.65 \% \\ \text { F1: } & 17.19 \%\end{aligned}$

Table 5.1: Effectiveness results of the BASELINE according to PROBI corpus

Of the 2,616 sentences annotated as containing grammar errors, 691 have errors not covered by any CoGrOO rule. Some categories are not covered by any CoGrOO rule and are listed at Appendix B. Excluding these errors the recall would rise from $13.65 \%$ to $18.55 \%$ and $\mathrm{F} 1$ from $17.19 \%$ to $20.62 \%$.

Table 5.2 detail effectiveness information for each error category of PROBI Corpus. 


\begin{tabular}{ccccccc}
\hline Category & Precision & Recall & F1 & Target & True positives & False positives \\
\hline cra & $76.98 \%$ & $25.19 \%$ & $37.96 \%$ & 385 & 97 & 29 \\
mal & $66.67 \%$ & $20.00 \%$ & $30.77 \%$ & 10 & 2 & 1 \\
cop & $72.73 \%$ & $17.14 \%$ & $27.75 \%$ & 140 & 24 & 9 \\
con & $26.78 \%$ & $22.22 \%$ & $24.29 \%$ & 441 & 98 & 268 \\
pro & $100.00 \%$ & $11.27 \%$ & $20.25 \%$ & 71 & 8 & 0 \\
aha & $36.36 \%$ & $13.79 \%$ & $20.00 \%$ & 29 & 4 & 7 \\
adv & $85.71 \%$ & $11.11 \%$ & $19.67 \%$ & 54 & 6 & 1 \\
cmt & $100.00 \%$ & $9.68 \%$ & $17.65 \%$ & 31 & 3 & 0 \\
sem & $66.67 \%$ & $9.52 \%$ & $16.67 \%$ & 21 & 2 & 1 \\
cov & $10.87 \%$ & $18.51 \%$ & $13.69 \%$ & 562 & 104 & 853 \\
cjc & $100.00 \%$ & $6.67 \%$ & $12.50 \%$ & 15 & 1 & 0 \\
reg & $80.00 \%$ & $3.17 \%$ & $6.11 \%$ & 126 & 4 & 1 \\
det & $100.00 \%$ & $2.86 \%$ & $5.56 \%$ & 35 & 1 & 0 \\
ver & $100.00 \%$ & $2.78 \%$ & $5.41 \%$ & 72 & 2 & 0 \\
lex & $100.00 \%$ & $1.67 \%$ & $3.28 \%$ & 60 & 1 & 0
\end{tabular}

Table 5.2: Effectiveness results for each error category of BASELINE according to PROBI corpus

\subsection{Metro Corpus}

The Metro corpus includes 781 sentences, 53 of these sentences were annotated as grammatically incorrect. BASELINE selected 83 sentences as containing grammar errors, but only 16 of these were correctly selected according to the corpus annotation. Table 5.3 presents a summary of the results.

$$
\begin{aligned}
\text { Sentences: } & 781 \\
\text { Target: } & 53 \\
\text { Selected: } & 83 \\
\text { True positives: } & 16 \\
\text { Precision: } & 19.28 \% \\
\text { Recall: } & 30.19 \% \\
\text { F1: } & 23.53 \%
\end{aligned}
$$

Table 5.3: Effectiveness results of BASELINE according to Metro corpus

Table 5.4 detail effectiveness for each error category.

\subsection{Bosque}

The Brazilian Portuguese part of Bosque corpus includes 4,212 professionally edited sentences. BASELINE selected 245 sentences as containing grammar errors and all should be considered false positives.

The evaluation also includes effectiveness information for each error category: 


\begin{tabular}{ccccccc}
\hline Category & Precision & Recall & F1 & Target & True positives & False positives \\
\hline ren & $100.00 \%$ & $100.00 \%$ & $100.00 \%$ & 1 & 1 & 0 \\
cjc & $100.00 \%$ & $100.00 \%$ & $100.00 \%$ & 1 & 1 & 0 \\
cra & $71.43 \%$ & $62.50 \%$ & $66.67 \%$ & 8 & 5 & 2 \\
reg & $100.00 \%$ & $18.18 \%$ & $30.77 \%$ & 11 & 2 & 0 \\
con & $18.75 \%$ & $75.00 \%$ & $30.00 \%$ & 8 & 6 & 26 \\
ptn & $100.00 \%$ & $6.25 \%$ & $11.76 \%$ & 16 & 1 & 0 \\
ali & $0.00 \%$ & $0.00 \%$ & $0.00 \%$ & 1 & 0 & 0 \\
cov & $0.00 \%$ & $0.00 \%$ & $0.00 \%$ & 4 & 0 & 39 \\
det & $0.00 \%$ & $0.00 \%$ & $0.00 \%$ & 2 & 0 & 0 \\
res & $0.00 \%$ & $0.00 \%$ & $0.00 \%$ & 1 & 0 & 0
\end{tabular}

Table 5.4: Effectiveness results for each error category of BASELINE according to Metro corpus

Sentences: $\quad 4,212$

False positives: 245

Table 5.5: Effectiveness results of CoGrOO 3.1.2 according to Bosque corpus

\begin{tabular}{cc}
\hline Category & False positives \\
\hline adv & 2 \\
aha & 2 \\
con & 134 \\
cop & 1 \\
cov & 94 \\
cra & 5 \\
ger & 6 \\
ptn & 1
\end{tabular}

Table 5.6: Effectiveness results for each error category of CoGrOO 3.1.2 according to Probi corpus

\subsection{CoGrOO Community Corpus}

CoGrOO Community Corpus (Section 3.4) includes 378 sentences, which 104 are flagged as grammatically incorrect. The corpus is composed by sentences submitted by the grammar checker users, sentences which either caused a false positive or a false negative. The effectiveness of the grammar checker in this corpus, at least in the initial evaluation, is expected to be quite low.

The BASELINE selected 158 sentences as containing grammar errors, but only 4 of those were correctly selected according to the corpus annotation. Table 5.7 presents a summary of the results, and Table 5.8 details the effectiveness information for each error category of CoGrOO Community Corpus. 
Sentences: 378

Target: 104

Selected: 158

True positives: 4

Precision: $2.53 \%$

Recall: $3.85 \%$

F1: $3.05 \%$

Table 5.7: Effectiveness results of the BASELINE according to CoGrOO Community corpus

\begin{tabular}{ccccccc}
\hline Category & Precision & Recall & F1 & Target & True positives & False positives \\
\hline cra & $25.00 \%$ & $9.09 \%$ & $13.33 \%$ & 11 & 1 & 3 \\
con & $2.14 \%$ & $12.00 \%$ & $3.64 \%$ & 25 & 3 & 137 \\
adv & $0.00 \%$ & $0.00 \%$ & $0.00 \%$ & 1 & 0 & 2 \\
aha & $0.00 \%$ & $0.00 \%$ & $0.00 \%$ & 3 & 0 & 5 \\
ali & $0.00 \%$ & $0.00 \%$ & $0.00 \%$ & 1 & 0 & 0 \\
cop & $0.00 \%$ & $0.00 \%$ & $0.00 \%$ & 5 & 0 & 0 \\
cov & $0.00 \%$ & $0.00 \%$ & $0.00 \%$ & 40 & 0 & 1 \\
ger & $0.00 \%$ & $0.00 \%$ & $0.00 \%$ & 0 & 0 & 4 \\
lex & $0.00 \%$ & $0.00 \%$ & $0.00 \%$ & 1 & 0 & 0 \\
ort & $0.00 \%$ & $0.00 \%$ & $0.00 \%$ & 4 & 0 & 0 \\
ptn & $0.00 \%$ & $0.00 \%$ & $0.00 \%$ & 2 & 0 & 0 \\
reg & $0.00 \%$ & $0.00 \%$ & $0.00 \%$ & 6 & 0 & 1 \\
ren & $0.00 \%$ & $0.00 \%$ & $0.00 \%$ & 0 & 0 & 1 \\
sem & $0.00 \%$ & $0.00 \%$ & $0.00 \%$ & 2 & 0 & 0 \\
ver & $0.00 \%$ & $0.00 \%$ & $0.00 \%$ & 3 & 0 & 0
\end{tabular}

Table 5.8: Effectiveness results for each error category of BASELINE according to CoGrOO Community corpus

\subsection{Conclusion}

The poor recall of BASELINE in the PROBI corpus was expected because, even considering only the error categories covered by the current version of the grammar checker, it would require a substantial number of new rules to cover all errors present in this corpus. The poor precision and recall in Community corpus were expected as well, because this corpus is formed by failures reported by the grammar checker users, and the issues in the sentences was not addressed yet.

The worst result is related to the low precision in the categories noun $(\mathrm{CON})$ and verb (COV) agreement categories, below $30 \%$ in the experiments with an annotated corpus. The issues with these two categories should be addressed with higher priority. 


\section{Chapter 6}

\section{NLP Development and Evaluation}

The first section of this chapter describes some initial improvements in the grammar checker code, which consists almost exclusively of bug corrections that were detected with a code reviewing process. These code changes already allowed for some improvements. Subsequently, the other sections describe the development and evaluation of each individual module, followed by experiments that measure the impact of the new module in the grammar checker effectiveness.

This is quite a long chapter, and it is advised to the readers to skip over the sections which are not related to their personal interests.

\subsection{Code Changes}

During the initial evaluation carried out, which was reported in Section 5, it became clear that the most important problems of CoGrOO 3.2.1 are related to the rules from noun $(\mathrm{CON})$ and verb (COV) agreement categories. With some code analysis and debugging, important issues were spotted:

Data Structures There was no data structures to represent the Shallow Parser annotations, which were saved as an attribute of Token. This simplification was causing the search for syntactic errors, mainly subject-verb agreement, to likely fail.

Equals Implementation The Rules Applier module relies a great deal on the Java equal method, implemented in each class that represents data, for example the Token, Chunk and Tag classes, but some implementation of equals was not complete and could cause comparison failures.

Strict Featurization Consider the example phrase A famosa artista (The famous artist). The word artista is a noun with two genders, meaning both masculine and feminine classifications would be correct, but a contextual featurizer would tend to classify it 
as feminine because of the leading feminine article and the feminine adjective famosa (famous). In some cases, the featurizer could opt for classifying artista as masculine, and by doing this in the example phrase, it would cause a false positive.

The grammar checker code was modified to address the described issues. For the first, some architecture changes were carried out to introduce a new data structure called SyntacticChunk, which represents the output of a shallow parser. For the second issue all equal method implementations were revised and improved.

To address the the strict featurization issue, a post processing module were introduced. This module uses a dictionary to check if a word has two genders or two numbers. If yes it modifies the feature tag to add this information. The featurizer would be less precise, but would cause less false positives.

\subsubsection{Evaluation}

The experiment consisted of evaluating the modified grammar checker, from now on named $N E W$, using the error annotated corpora. The results are compared to the one obtained by the original grammar checker, named BASELINE. Table 6.1 summarizes the results.

\begin{tabular}{|c|c|c|c|c|c|c|c|}
\hline & \multicolumn{3}{|c|}{ Metro } & \multicolumn{3}{|c|}{ Probi } & Bosque \\
\hline Experiment & Target & $\mathrm{TP}$ & $\mathrm{FP}$ & Target & $\mathrm{TP}$ & $\mathrm{FP}$ & $\mathrm{FP}$ \\
\hline BASELINE & & 16 & 67 & & 357 & 1181 & 395 \\
\hline NEW & 53 & 16 & $\begin{array}{r}32 \\
+2 \\
-37\end{array}$ & 2616 & $\begin{array}{r}333 \\
+10 \\
-34\end{array}$ & $\begin{array}{r}588 \\
+48 \\
-641\end{array}$ & $\begin{array}{r}245 \\
+28 \\
-178\end{array}$ \\
\hline
\end{tabular}

Table 6.1: Evaluation results comparing the effect of the proposed changes. BASELINE is CoGrOO 3.2.1 and $N E W$ the grammar checker that includes the proposed changes. The numbers in italic refers to the changes with relation to the BASELINE. For example, +2 in the column Metro FP means that two new false positives were found and -37 in the same column that 37 entries were removed.

The number of false positives dropped expressively: $52 \%$ in Metro corpus, $50 \%$ in Probi and $38 \%$ in Bosque. The number of true positives changed only in Probi by $7 \%$ less true positives. The following paragraphs detail these results.

Table 6.2 provides details on how the changes affected the evaluation with the Metro corpus. All false positives removed were caused by rules of noun (CON) and verb (COV) agreement categories.

From Table 6.3 we can see that in the Probi corpus other rule categories were also affected by the changes. It is important to note why some true positives were lost in verifying whether there were any side effects.

For example, BASELINE found noun agreement true positives that could not be found by the modified version. In Example 6.1, the rule that matched was adjective, masculine, 


\begin{tabular}{|c|c|c|}
\cline { 2 - 3 } \multicolumn{1}{c|}{} & BASELINE & NEW \\
\cline { 2 - 3 } \multicolumn{1}{c|}{} & FP & FP \\
\hline con & 26 & 19 \\
\hline cov & 39 & 11 \\
\hline
\end{tabular}

Table 6.2: Detailed evaluation results comparing the effect of the proposed changes to the Metro corpus. The number of true positives did not change.

\begin{tabular}{|c|c|c|c|c|}
\cline { 2 - 5 } \multicolumn{1}{c|}{} & \multicolumn{2}{|c|}{ BASELINE } & \multicolumn{2}{c|}{ NEW } \\
\cline { 2 - 5 } \multicolumn{1}{c|}{} & TP & FP & TP & FP \\
\hline con & 98 & 268 & 93 & 231 \\
\hline cov & 104 & 853 & 85 & 297 \\
\hline cop & 24 & 9 & 25 & 9 \\
\hline aha & 4 & 7 & 4 & 6 \\
\hline cra & 97 & 29 & 96 & 28 \\
\hline ger & 0 & 4 & 0 & 6 \\
\hline
\end{tabular}

Table 6.3: Detailed evaluation results comparing the effect of the proposed changes in Probi corpus.

plural followed by noun, masculine, singular. With the feature generalization, the two gender token mel hores (better) changed to neutral and the rule stopped matching. The same issue was found with the entries PROBI-con-105-27, PROBI-con-105-28 and a number of other entries. To fix it the rule should be improved.

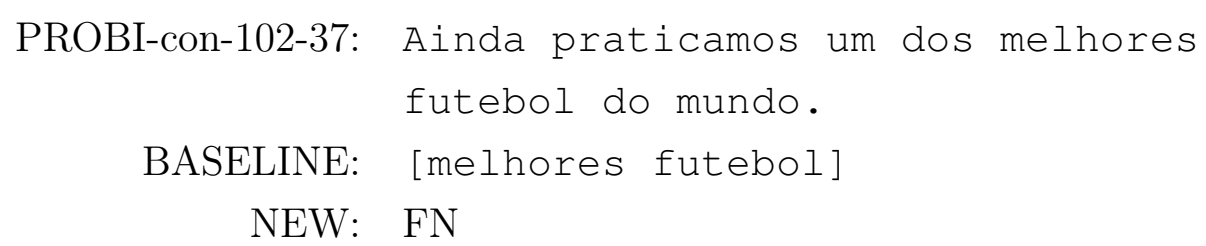

Example 6.2 illustrates another issue introduced with the changes. The token menina (girl) is a singular noun, but its number was erroneously generalized to neutral. Investigating it further, what caused the error was an error in the dictionary entry, in which menina was also classified as a plural noun, letting the POS Tagger accept this classification.

\section{PROBI-con-102-61: As menina bonitas. \\ BASELINE: [menina bonitas] \\ NEW: FN}

Example 6.3 is an interesting case. The true positive pointed out by the BASELINE was in fact a false positive. The real error of this example is cujas cenas, but the BASELINE did not implement any rule that could match it. The BASELINE erroneously found an issue 
between the predicate and the subject.

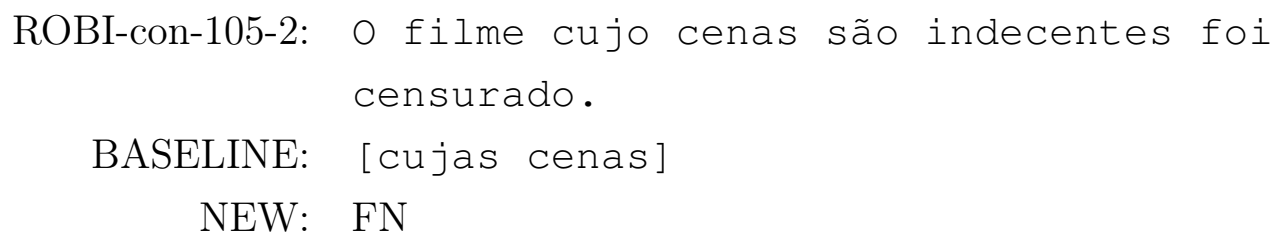

Example 6.4 shows a true positive detected by BASELINE, but after the changes it is a false negative. The related rule searches for an à followed by a singular masculine noun. The two number tokens lápis (pencil) were initially classified as singular because of the context, but the new code changed it to neutral and the rule no longer matched. Future research may analyze whether the rule can be changed to match even for neutral nouns like lápis.

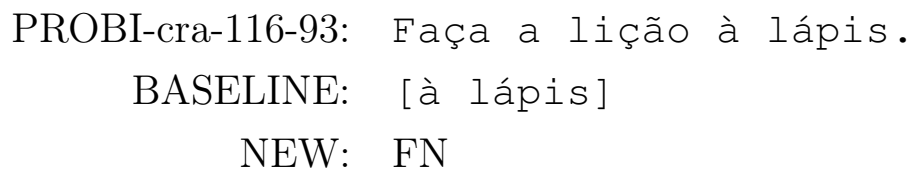

\subsubsection{Conclusion}

The changes were positive and improved the grammar checker in terms of number of false positives. The number of true positives declined slightly in Probi corpus, but analyzing a sample of the errors proved that the real issue is not the changes, but problem related to the rules or to the dictionary.

These changes will be incorporated into the BASELINE grammar checker and used during the evaluation of the other modules. 


\subsection{Sentence Boundary Detector}

The sentence boundary detector module, as described in Section 3.1.1, is responsible for identifying the limits of sentences based on punctuation marks.

The proposal improvement for this module is to replace the one included in version 3 of the grammar checker with a new one, which was created from scratch using the latest Apache OpenNLP version.

Apache OpenNLP implements the algorithm proposed by Ratnaparkhi (1998, sec. 4.3), summarized as follows: it scans the text for end of sentence (EOS) characters and collects information from the token containing the potential sentence boundary, as well as the contextual information for the tokens immediately to the left and to the right. The outcomes are either yes or no. The former indicates that the potential EOS character is a sentence boundary, while the latter denotes that it is not.

An initial customization to Apache OpenNLP code was to include extra EOS characters to conform with those occurring in the Floresta corpora, as decribed in (Afonso et al., 2001). The characters ; : : (, ), «, », " and " were added to the default: ., ! and ?.

\subsubsection{Default Contextual Predicates}

The default Sentence Boundary Detector implementation relies on a number of contextual templates. Lets define Candidate as the token containing the EOS character. Prefix is the portion of the Candidate that precedes the EOS character, and suffix the portion that follows it.

The contextual templates have the following form:

- The prefix and its features;

- The suffix and its features;

- The previous token and its features;

- The following token and its features;

- The end of sentence character;

- Whether the candidate is an abbreviation.

The features of the prefix, suffix, previous token and following token includes:

- Any special character in it;

- If first letter is upper case;

- Its length; 
- Whether it is an abbreviation.

The contextual predicates ${ }^{1}$ are the combination of the contextual template and the actual data. For example, for the first potential EOS character sr. in the sentence from Example 6.5, the contextual predicates would include: Prefix $=\mathrm{sr}$, Prefix $=N U L L$, Feature $=A B B$ etc.

O sr. Mendonça chegou.

\section{Evaluation}

A set of 10-fold cross-validation experiments were carried out using the default contextual predicates. Each experiment combines:

- An algorithm (MAXENT or PERCEPTRON)

- A corpus - Floresta Virgem CETENFolha (VCF), Bosque CETENFolha (CF) and Amazônia (AMA)

- Whether to include or not the abbreviation dictionary (ABB)

Each of the 12 combinations was evaluated using different cutoff values: $0,2,4,8,16$, $32,64,128,256$ and 512 .

The highest $F_{1}$ score was $98.95 \%$ obtained by a Perceptron model trained with VCF, cutoff zero, using the abbreviation dictionary. Table 6.4 summarizes the experiment results.

Figure 6.1 makes it easier to visualize the behavior of the $F_{1}$ score for different sentence boundary detector configurations. The $F_{1}$ score of models trained with VCF surpasses the score from those trained with CF and AMA by at least $2 \%$ in any configuration. AMA is composed of unrevised documents with different language styles. This would explain the low score obtained, but on the other hand, it is a $275 \mathrm{k}$ sentences corpus, causing $F_{1}$ to maintain a high value even increasing the cutoff. VCF and CF are both composed of professionally revised journal articles, therefore it is more homogeneous compared to AMA. This could be one reason for a higher $F_{1}$ compared to AMA. The differential of VCF is its size, 96k sentence compared to $4 \mathrm{k}$ from $\mathrm{CF}$.

The impact of including the abbreviation dictionary was fairly higher while evaluating with CF, but it also improved slightly while evaluating with AMA and VCF. The difference was more accentuated for high cutoff values: with reduced predicates, either caused by the use of a small corpus or by a high cutoff value, the abbreviation dictionary becomes more important because the model does not need to rely on corpus observation to determine whether a token is an abbreviation or not.

Perceptron models benefit from including all features by setting zero cutoff and the effectiveness declines while increasing cutoff. In contrast, a Maxent model suffers with a zero

\footnotetext{
${ }^{1}$ Apache OpenNLP mixes the concept of Features and Contextual Predicates.
} 


\begin{tabular}{|c|c|c|c|c|c|c|c|c|c|c|c|c|}
\hline 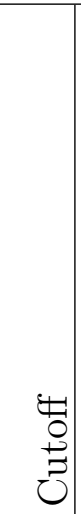 & 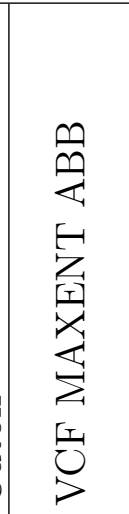 & 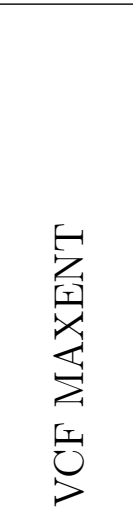 & 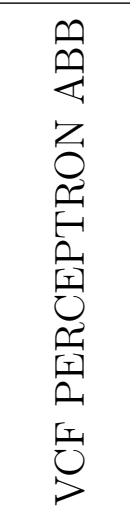 & 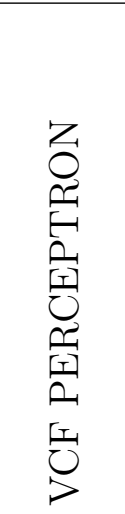 & 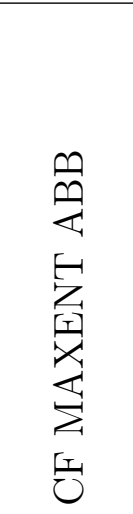 & 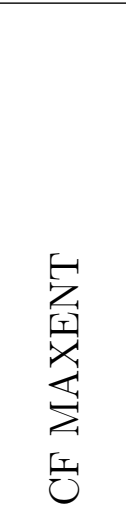 & 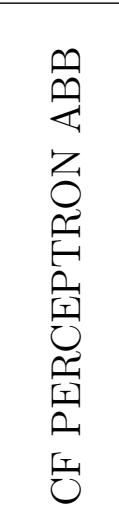 & 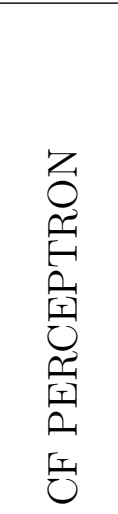 & 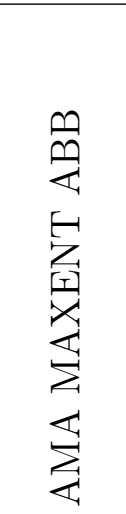 & 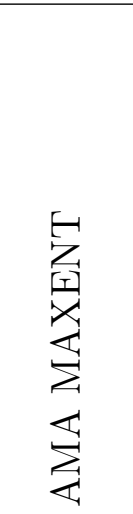 & 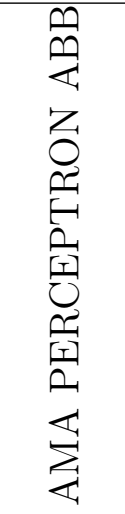 & 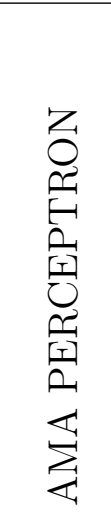 \\
\hline 0 & 8.48 & 3.34 & 3.95 & 3.93 & 5.70 & .28 & 958 & .67 & 93.81 & 93.81 & 95.12 & 5.07 \\
\hline 2 & 98.65 & 98.65 & 70 & 78 & 0611 & 66 & 10 & 8 & 03 & .01 & 4.96 & 86 \\
\hline 4 & 98.72 & 98.71 & 35 & 98 & 96 & & 94 & 7 & 94 & & 93.94 & 94.01 \\
\hline 8 & 98.75 & 98.75 & 98.71 & & Q & & 94.68 & 94.35 & 94.12 & & 94.27 & 94.27 \\
\hline 16 & 98.72 & 98 & 91 & & 9 & 94 & 26 & 93.40 & 94.14 & & 93.97 & 94.11 \\
\hline 32 & & & 93 & & & & & & & & 93.93 & 9.9 .90 \\
\hline 64 & 98.19 & 98.23 & 71 & & 93 & 93 & 92.81 & 92.43 & 93 & 93 & 93.60 & 93.83 \\
\hline 128 & 97.90 & & 8.47 & & & & 89.65 & 89.65 & 93 & & 93.55 & 93.70 \\
\hline 256 & 97.72 & 97.31 & 8.10 & & & & & & & & 93.26 & 93.51 \\
\hline 512 & 7.83 & 97.36 & 98.07 & 97.38 & 90.72 & 90.72 & 75.81 & 75.81 & 93.61 & 93.21 & 93.30 & 93.50 \\
\hline
\end{tabular}

Table 6.4: 10-fold cross validation results for different configurations of sentence boundary detector. The highest $F_{1}$ score of each configuration is in bold, and the overall highest score is underlined.

cutoff and performs better, increasing it. Perceptron models trained with a larger corpus exceed Maxent models in terms of $F_{1}$, but Maxent models were better with a small corpus in this experiment. These empirical findings were also noticed by Collins (2002), and the explanation of the curve behavior is left for future studies.

Although the $F_{1}$ score is the selection criterion, it is important to verify the model size. As shown in Table 6.5, the largest models were those trained with the AMA corpus, followed by VCF and CF. Models that include the abbreviation dictionary are usually larger because the dictionary is bundled in the model. The models that had best $F_{1}$ score, those trained with VCF, have a reasonable size.

\subsubsection{Additional Context Predicates}

While analyzing the errors using the default context predicates, a set of additional context predicates were proposed. They check fine grained features of tokens which contain an EOS character and were inspired by the Tokenizer default contextual predicates. They are:

- The last character before EOS and its features;

- The penultimate character before EOS and its features;

- The character next to EOS and its features; 


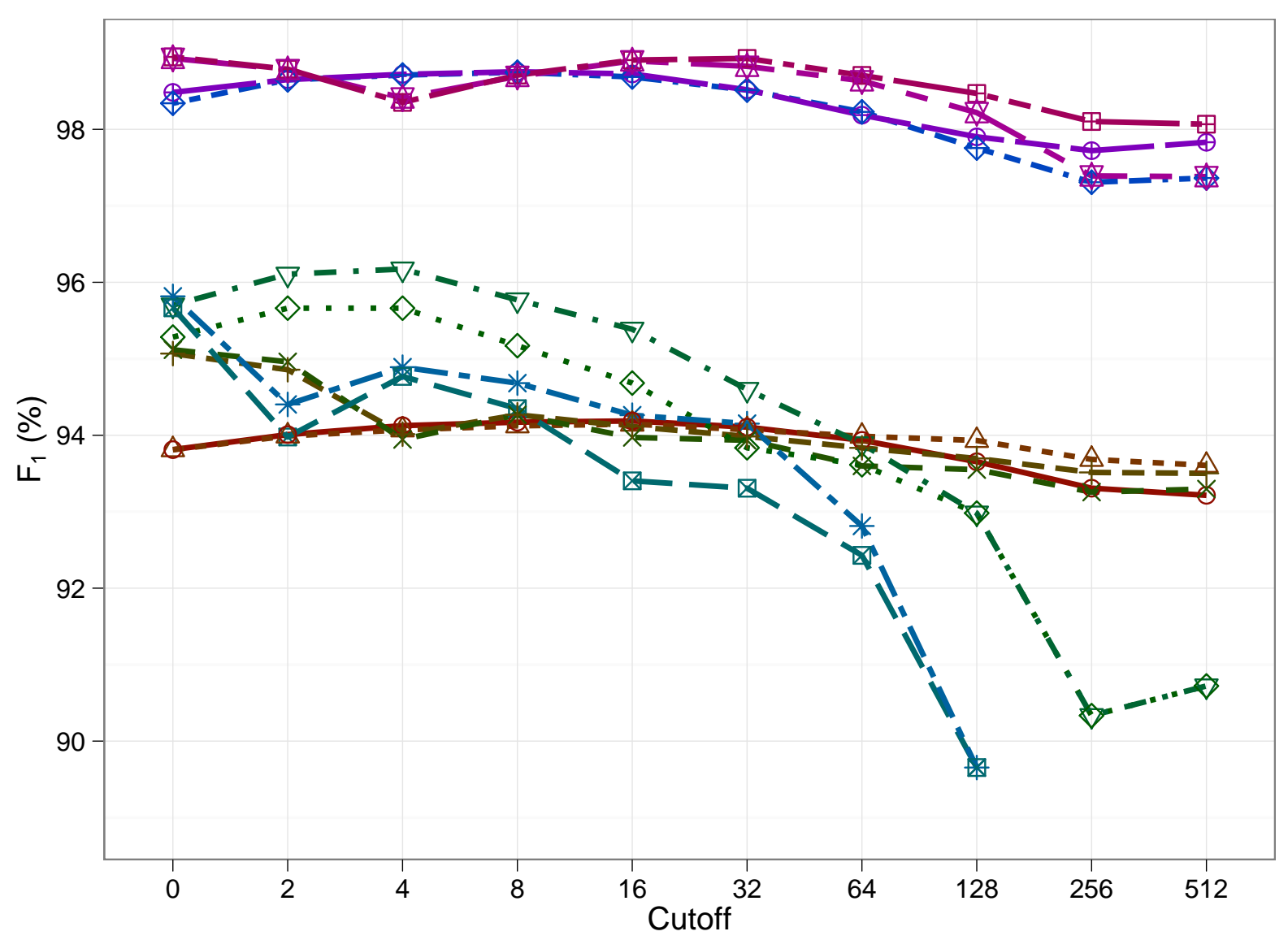

\begin{tabular}{|c|c|c|c|c|}
\hline \multirow{6}{*}{ Experiments } & $\theta$ & \multirow{6}{*}{$\begin{array}{l}\text { AMA MAXENT } \\
\text { AMA MAXENT ABB } \\
\text { AMA PERCEPTRON } \\
\text { AMA PERCEPTRON ABB } \\
\text { CF MAXENT } \\
\text { CF MAXENT ABB }\end{array}$} & $\approx$ & \multirow{3}{*}{$\begin{array}{l}\text { CF PERCEPTRON } \\
\text { CF PERCEPTRON ABB } \\
\text { VCF MAXENT }\end{array}$} \\
\hline & 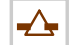 & & - * & \\
\hline & 7 & & $\Leftrightarrow$ & \\
\hline & $x$ & & 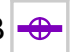 & VCF MAXENT ABB \\
\hline & $\diamond$ & & 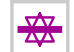 & VCF PERCEPTRON \\
\hline & $\nabla$ & & 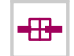 & VCF PERCEPTRON ABB \\
\hline
\end{tabular}

Figure 6.1: Distribution of the $F_{1}$ score according to the sentence detector configuration and the cutoff.

Character features includes:

- The character itself;

- Whether it is alphanumeric;

- Whether it is upper case;

- Whether it is a digit;

- Whether it is a white space;

- If it is a symbol, the type of symbol (i.e. quote, EOS, relative EOS etc.). 


\begin{tabular}{|c|r|r|}
\hline Experiment & $F_{1}(\%)$ & Size $(\mathrm{kB})$ \\
\hline VCF PERCEPTRON ABB & 98.947 & 64 \\
VCF PERCEPTRON & 98.928 & 63 \\
VCF MAXENT ABB & 98.753 & 59 \\
VCF MAXENT & 98.746 & 57 \\
CF MAXENT ABB & 96.173 & 12 \\
CF PERCEPTRON ABB & 95.816 & 13 \\
CF PERCEPTRON & 95.666 & 12 \\
CF MAXENT & 95.661 & 29 \\
AMA PERCEPTRON ABB & 95.117 & 301 \\
AMA PERCEPTRON & 95.068 & 302 \\
AMA MAXENT & 94.187 & 150 \\
AMA MAXENT ABB & 94.144 & 152 \\
\hline
\end{tabular}

Table 6.5: Size of the best model of each experiment. Lines are sorted by $F_{1}$.

All the default contextual predicates were kept.

\section{Evaluation}

Another set of 10 -fold cross-validation experiments were carried out using the additional contextual predicates; this time they combined:

- An algorithm (MAXENT or PERCEPTRON);

- The Floresta Virgem CETENFolha (VCF);

- Whether to include or not the abbreviation dictionary (ABB);

- Whether to use the additional contextual predicates or not (ACP).

Since models created with AMA and CF demonstrated poor effectiveness compared to models created with VCF, the first two corpora were not included in this evaluation.

The highest $F_{1}$ score was $99.24 \%$ obtained by a Perceptron model trained with VCF, cutoff zero, tied with the perceptron model that includes an abbreviation dictionary, which scored $99.23 \%$. Table 6.6 summarizes the experiment results.

Figure 6.2 can be used to visualize the behavior of the $F_{1}$ score for the different configurations. Additional context predicates increased the $F_{1}$ score by $0.3 \%$ or $0.4 \%$, according to the configuration, but curves kept the same shape, thus the analysis from default contextual predicates still applies.

The impact of including the abbreviation dictionary was fairly higher while evaluating with CF, but it slightly improved while evaluating with AMA and VCF. The difference was more accentuated for high cutoff values. With reduced features, either caused by the use of a small corpus or by a high cutoff, the abbreviation dictionary becomes more important 


\begin{tabular}{|c|c|c|c|c|c|c|c|c|}
\hline \multirow[b]{2}{*}{ 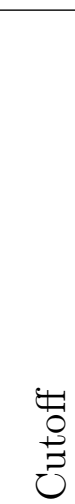 } & $\stackrel{\oplus}{\varphi}$ & & & & 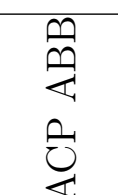 & 己s & $\stackrel{\varphi}{\eta}$ & \multirow[b]{2}{*}{ 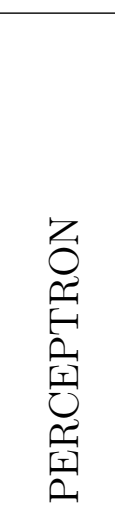 } \\
\hline & 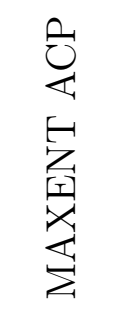 & 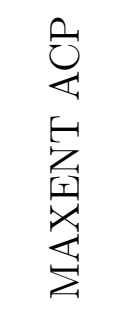 & 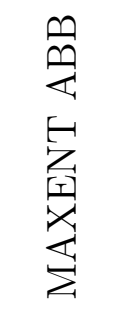 & 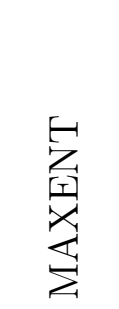 & 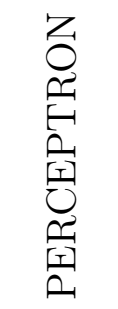 & 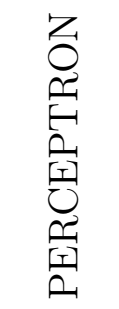 & 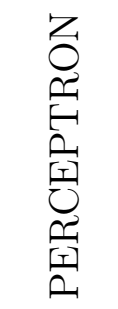 & \\
\hline 0 & 98.92 & 98.92 & 98.48 & 98.34 & 99.23 & 99.24 & 98.95 & 98.93 \\
\hline 2 & 99.03 & 99.05 & 98.65 & 98.65 & 99.06 & 99.10 & 98.79 & 98.78 \\
\hline 4 & 99.11 & 99.12 & 98.72 & 98.71 & 98.67 & 98.60 & 98.35 & 98.41 \\
\hline 8 & 99.09 & 99.09 & 98.75 & 98.75 & 98.36 & 98.39 & 98.71 & 98.69 \\
\hline 16 & 99.07 & 99.05 & 98.72 & 98.69 & 98.78 & 98.78 & 98.91 & 98.90 \\
\hline 32 & 98.94 & 98.94 & 98.52 & 98.51 & 98.86 & 98.86 & 98.93 & 98.82 \\
\hline 64 & 98.77 & 98.73 & 98.19 & 98.23 & 98.77 & 98.81 & 98.71 & 98.63 \\
\hline 128 & 98.51 & 98.53 & 97.90 & 97.76 & 98.83 & 98.99 & 98.47 & 98.22 \\
\hline 256 & 98.40 & 97.99 & 97.72 & 97.31 & 98.68 & 98.61 & 98.10 & 97.39 \\
\hline 512 & 98.33 & 97.78 & 97.83 & 97.36 & 98.42 & 98.30 & 98.07 & 97.38 \\
\hline
\end{tabular}

Table 6.6: 10-fold cross validation results for configurations of a sentence boundary detector using the additional (ADDPRED) and the default context predicates. The highest $F_{1}$ score of each configuration is in bold, and the overall highest score is underlined.

because the model does not need to rely on corpus observation to determine whether a token is an abbreviation or not.

Perceptron models benefit from including all features by setting zero cutoff and the effectiveness declines while increasing cutoff. In contrast, a Maxent model suffers with a zero cutoff and performs better increasing it. Perceptron models trained with a bigger corpus exceed Maxent models in terms of $F_{1}$, but Maxent models were better with a small corpus in this experiment. This empirical result was also noticed by (Collins, 2002) and the explanation of the curve behavior is left for future studies.

Table 6.7 lists the size of the best model of each experiment with the VCF corpus. It is possible to notice that Maxent models with additional context predicates are larger than any other configuration trained with the same corpus. The models with the best $F_{1}$ scores, the Perceptron models with and without abbreviation dictionaries, have reasonable sizes.

\subsubsection{Previous Work}

(Ratnaparkhi, 1998) reports an accuracy of $98.8 \%$ in a Sentence Boundary Detector trained with a 40k-sentence English corpus using the Maxent framework. It includes the default contextual predicates described in Section 6.2.1, some additional language specific contextual predicates and an abbreviation dictionary. 


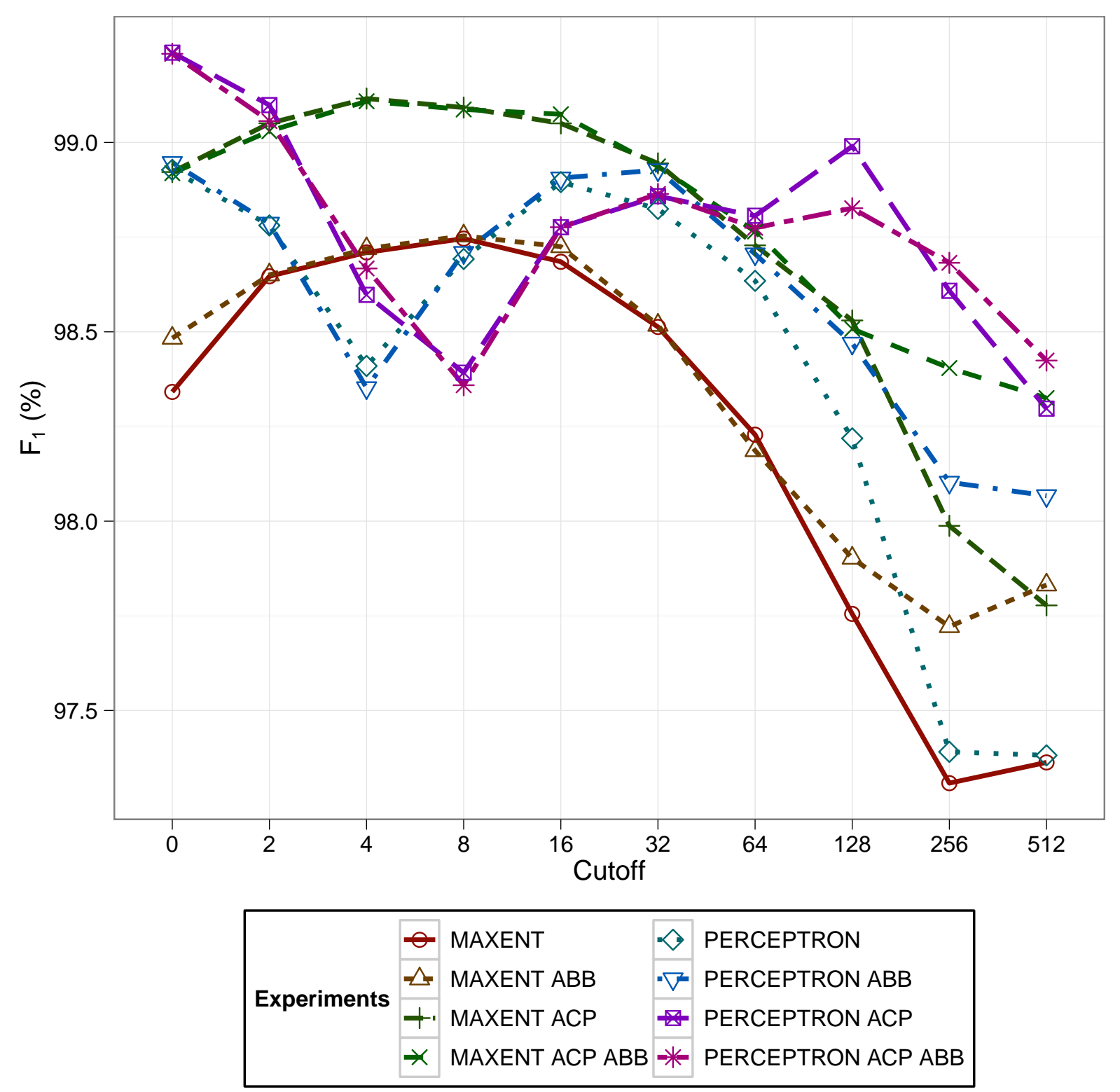

Figure 6.2: Distribution of the $F_{1}$ score according to the sentence detector configuration and the cutoff. All experiments were with a VCF corpus.

The Portuguese rule based Sentence Boundary introduced by (Silva, 2007) scored 99.94 of $F_{1}$ over a $12 \mathrm{k}$ sentences corpus. A very good value compared to the one achieved by the present work, but, according to (Silva, 2007, p. 73), it is to be expected because a rule based approach can fine-tuned to the orthographic conventions of the language.

There is no effectiveness information in the literature for this module in any previous CoGrOO version.

\subsubsection{Evaluating the Grammar Checker}

This evaluation consists of replacing the Sentence Boundary Detector module of CoGrOO 3.1.2 with the candidate versions and verifying its effectiveness using corpora annotated with 


\begin{tabular}{|c|c|r|}
\hline Experiment & $F_{1}(\%)$ & Size $(\mathrm{kb})$ \\
\hline PERCEPTRON ACP & 99.237 & 60 \\
PERCEPTRON ACP ABB & 99.234 & 61 \\
MAXENT ACP & 99.116 & 138 \\
MAXENT ACP ABB & 99.109 & 140 \\
PERCEPTRON ABB & 98.947 & 64 \\
PERCEPTRON & 98.928 & 63 \\
MAXENT ABB & 98.753 & 59 \\
MAXENT & 98.746 & 57 \\
\hline
\end{tabular}

Table 6.7: Size of the best model of each experiment. Lines are sorted by $F_{1}$.

grammar errors.

\begin{tabular}{|c|c|c|c|c|c|c|c|}
\hline & \multicolumn{3}{|c|}{ Metro } & \multicolumn{3}{|c|}{ Probi } & Bosque \\
\hline Experiment & Target & $\mathrm{TP}$ & $\mathrm{FP}$ & Target & $\mathrm{TP}$ & $\mathrm{FP}$ & $\mathrm{FP}$ \\
\hline$\overline{\text { BASELINE }}$ & \multirow{6}{*}{53} & 16 & 32 & \multirow{6}{*}{2616} & 333 & 588 & 245 \\
\hline $\begin{array}{l}\text { VCF PERCEPTRON ACP } 0 \\
\text { VCF PERCEPTRON ACP ABB } 0 \\
\text { VCF MAXENT ACP } 4 \\
\text { VCF MAXENT ACP ABB } 4 \\
\text { VCF PERCEPTRON ABB } 0 \\
\text { VCF PERCEPTRON } 0 \\
\text { VCF MAXENT ABB } 8 \\
\text { VCF MAXENT } 8\end{array}$ & & 16 & $\begin{array}{r}34 \\
+2\end{array}$ & & 333 & 588 & $\begin{array}{r}249 \\
+4\end{array}$ \\
\hline $\begin{array}{l}\text { CF MAXENT ACP ABB } 4 \\
\text { CF MAXENT ABB } 4\end{array}$ & & 16 & $\begin{array}{r}33 \\
+1\end{array}$ & & 333 & 588 & $\begin{array}{r}246 \\
+2 \\
-1\end{array}$ \\
\hline $\begin{array}{l}\text { CF PERCEPTRON ACP } 2 \\
\text { CF PERCEPTRON ACP ABB } 2\end{array}$ & & 16 & $\begin{array}{r}34 \\
+2\end{array}$ & & 333 & 588 & $\begin{array}{r}246 \\
+3 \\
-2\end{array}$ \\
\hline $\begin{array}{l}\text { CF MAXENT ACP } 2 \\
\text { CF MAXENT } 2\end{array}$ & & 16 & $\begin{array}{r}33 \\
+1\end{array}$ & & 333 & 588 & $\begin{array}{r}247 \\
+3 \\
-1\end{array}$ \\
\hline $\begin{array}{l}\text { CF PERCEPTRON ABB } 0 \\
\text { CF PERCEPTRON } 0\end{array}$ & & 16 & $\begin{array}{r}34 \\
+2\end{array}$ & & 333 & 588 & $\begin{array}{r}247 \\
+3 \\
-1\end{array}$ \\
\hline
\end{tabular}

Table 6.8: Summary of the grammar checker evaluation using different configuration of the boundary sentence detector module. The number in the experiment name refers to the cutoff value used. The Bosque corpus does not include grammar error annotations. Signed numbers refer to removed (when negative) or added (when positive) entries.

The results are summarized in Table 6.8. The sections that follow below analyze the results in detail. 


\section{Metro Corpus}

No new true positives were found by introducing a new Sentence Boundary Detector, but the number of false positives changed. Some configurations caused one more false positives, others two.

$C F$ MAXENT ABB introduced one new false positive in entry MT40-2. The output 6.6 clarifies what happened. With BASELINE the sentence was divided in two because it handled erroneously the punctuation mark in 2.700 as a EOS. The new trained module fixed this error, but handling this sentence accurately in this level caused another error later in the grammar checker pipeline, possibly caused by the POS Tagger.

MT40-2: A Ouvidoria do Metrô já atendeu, neste ano,

2.700 ocorrências entre sugestões, elogios

e reclamações.

BASELINE: [A Ouvidoria do Metrô já atendeu, neste ano, 2.700], [ocorrências entre sugestões, elogios e reclamações.]

CF MAXENT: [A Ouvidoria do Metrô já atendeu, neste ano, 2.700 ocorrências entre sugestões, elogios e reclamações.]

Error: disagreement in [2.700 ocorrências] (pronoun + noun)

In addition to MT40-2, CF MAXENT ACP ABB introduced another false positive in entry MT24-3, reproduce in Example 6.7. BASELINE decided by splitting the sentence in 2-Verde (trecho, but the new configurations did not, and it caused a misclassification later in the pipeline.

MT40-2: (...) a ampliação da Linha 2-Verde(trecho Ana Rosa-Ipiranga) e a construção da Linha (...)

BASELINE: [a ampliação da Linha 2-Verde(trecho], [Ana Rosa-Ipiranga) e a construção da Linha]

CF MAXENT: [a ampliação da Linha 2-Verde(trecho Ana Rosa-Ipiranga) e a construção da Linha]

Error: disagreement in [2-Verde ( trecho] (determiner + noun)

All the other configurations presented similar results.

\section{Probi Corpus}

No changes for this corpus, mainly because its entries always contain only one sentence. 


\section{Bosque Corpus}

CF MAXENT caused three new false positives, but one false positive caused by BASELINE was removed.

The false positive related to entry CF753-2 was removed because the sentence was divided by BASELINE, but it should not have because Elvis: From Nashville To Memphis is part of the same sentence. Keeping it in the same sentence caused an error later in the grammar checker pipeline.

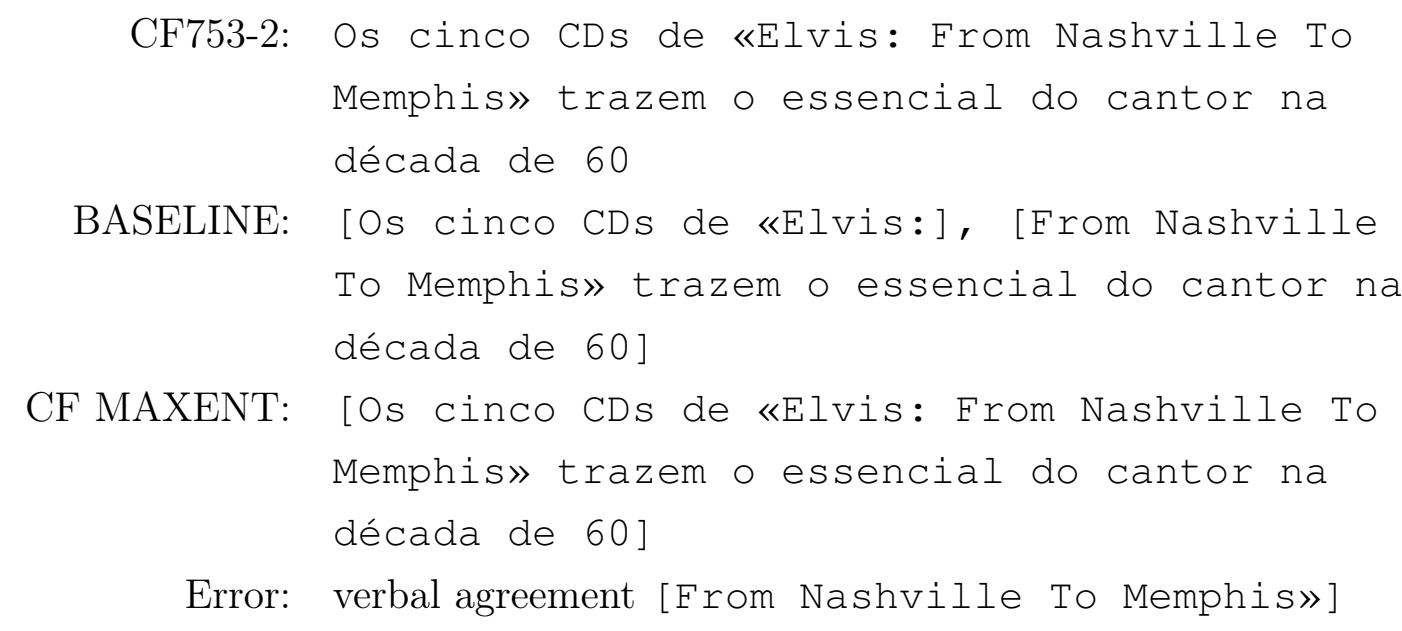

The other three false positives are related to the thousand separator in the corpus entries CF243-3, CF505-1 and CF626-3. They were 5.800, 2.100 and 3.600 respectively, and all were similar to the case described before in Example 6.6, were BASELINE decided that the number with a thousand separator was splitting the sentence.

All the other configurations trained with the CF corpus performed the same as $C F M A X$ ENT, except for CF MAXENT ABB that as example of BASELINE mistakenly divided the entry CF243-3 in the thousand separator.

All configurations trained with VCF were similar in this evaluation. They correctly handled the entry CF753-2 by allowing Elvis: From Nashville To Memphis in the same sentence, and they also properly handled the thousand separator in the entries CF243-3, CF505-1 and CF626-3.

In addition, the models trained with VCF caused one extra false positive in entry CF9467, detailed in Example 6.9. BASELINE was splitting the sentence, and models trained with VCF corpus kept it as one. This caused another error later in the pipeline, maybe, in 
Tokenizer and POS Tagger modules, leading to the false positive.

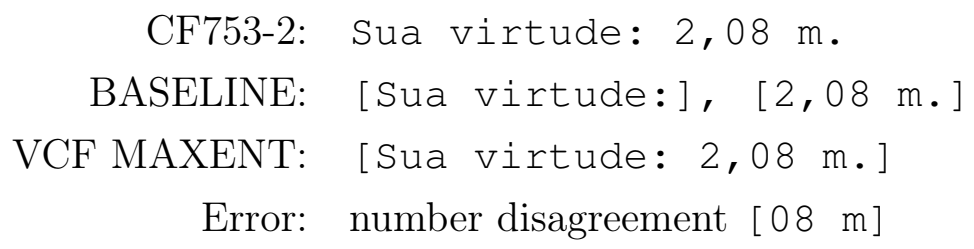

\subsubsection{Conclusion}

There are two main factors that should count while selecting the best Sentence Boundary Detector configuration: its $F_{1}$ score in the 10-fold cross-validation, and effectiveness of the grammar checker after switching the module with the new one.

The Perceptron algorithm proved superior to Maxent in these tasks in terms of $F_{1}$. Also the size and quality of the training corpus demonstrated to influence the results, and models trained with VCF archived higher $F_{1}$ score. Including or not the abbreviation dictionary was inconclusive in terms of $F_{1}$ to models trained with $\mathrm{VCF}$, but intuitively it should be better because it had a positive impact in models trained with a smaller corpus. Finally the additional contextual predicates had a positive impact.

In terms of the effectiveness of the grammar checker, a new Sentence Boundary Detector did not increase the number of true positives, even worse, at least at first glance, it increased the number of false positives. But after a detailed analysis most of the new false positives showed to be positive by having highlighted issues to be tackled in the next modules of the grammar checker pipeline.

Considering these two aspects, the following configuration was selected:

- Algorithm: Perceptron

- Abbreviation dictionary: yes

- Additional contextual predicates: yes

- Cutoff: zero

- Training corpus: Floresta Virgem CETENFolha

The new Sentence Boundary Detector will be incorporated to BASELINE and used during the evaluation of the other modules. 


\subsection{Tokenizer}

The tokenizer module is responsible for identifying token boundaries. For most tokenization decisions, this task is trivial because tokens are delimited by white spaces. However, there are also non-trivial cases in which there is no white spaces between tokens, for example when a punctuation mark follows a word.

The improvement proposed here is to replace CoGrOO 3.1.2 tokenizer module by one based on the latest version of Apache OpenNLP.

The Apache OpenNLP tokenizer algorithm works as follows:

- The module receives a sentence and divides it into token candidates using the white spaces;

- If the token candidates matches the alphanumeric pattern, it is a token (only if alphanumeric optimization was selected);

- If the token candidates do not match the alphanumeric pattern, the algorithm lets the machine learning component make the decision. In this case each character of the token candidate is tested as a token separator.

An initial customization to Apache OpenNLP was to extend its definition of alphanumeric pattern, that represented by the regular expression ^ $[\mathrm{A}-\mathrm{Za}-\mathrm{z} 0-9]+\$$, that does not cover tokens with accent markers. Figure 6.10 shows the adapted definition of the alphanumeric pattern.

$$
\text { ^ [0-9a-záãâàéêíóõôúüçA-ZÁãÂÀÉÊÉIóõôúÜÇ ] +\$ }
$$

\subsubsection{Default Contextual Predicates}

The machine learning algorithm is modeled as a decision problem that decides if a character of the token candidate is likely to divide the token in two or not. To decide this, Apache OpenNLP uses contextual predicates to collect features of each candidate, lets define the Candidate, the token being analyzed, and Index a character position of the candidate. Prefix is the portion of the candidate before the index, and suffix, the portion that follows it.

The contextual templates have the following form:

- The prefix;

- The suffix;

- The last character before index and its features;

- The penultimate character before index and its features; 
- The character next to index and its features;

Character features includes:

- The character itself;

- Whether it is an alphanumeric;

- Whether it is upper case;

- Whether it is a digit;

- Whether it is a white space;

- If it is a symbol, the type of symbol (i.e. quote, EOS, relative EOS, etc.).

The contextual predicates are the combination of the contextual template and the actual data. For example, for index $=3$ in the token from Example 6.11 the contextual predicates would include: Prefix $=200$, Suffix $=.000,00$, Previous character $=0$, Previous character type $=$ digit, etc.

$$
200.000,00
$$

\section{Corpora pre-processing}

Dealing with hyphenated words in Bosque CETENFolha and Floresta Virgem CETENFolha corpus is complex. Sometimes it will separate hyphenated words and other times not according to their semantic identity (Afonso, 2006). For example, hyphenated words that represents a complex unit, like sexta-feira (Friday), are never separated; on the other hand, clitics linked to a verb by a hyphen are always separated, for example degradar-se (deteriorate itself) will form two tokens: degradar -+ se.

Another case is when each component of the hyphenated word is part of a different phrase, for example in ex-presindente (former president), the prefix ex, depending on the context, will be part of an adjective phrase and presidente of a noun phase, and in this case it would appear as two tokens in the corpus. If both are part of the same phrase, they would be only one token.

This is a major issue while training the tokenizer component because further analysis is required to decide if a hyphenated word should be separated or not. To overcome this, the corpus was preprocessed and all hyphenated words were separated.

If in the corpus the hyphenated word was represented by one token, each of the words forming it was separated and the original POS tags was associated with it. If the hyphenated word was represented by more than one token, they were kept this way and the POS tags of each component were kept. 
CoGrOO 3.1.2 does not perform this pre-processing step. By introducing it in the new tokenizer, a lower effectiveness in the handling of hyphenated words by the POS Tagger of CoGrOO 3.1.2 is expected, but it will be addressed later with the development of the new POS Tagger in Section 6.6.

Another issue that required pre-processing was the complex named entities. Usually, named entities are formed by one or more words linked with an underscore forming one token, for example Marco Antonio. In this case the pre-processing consists of splitting in the underscore forming new tokens. But some named entities are more complex, composed of punctuation and quotation marks, sometimes they are complete sentences themselves, for example when the named entity is a book title, like in Example 6.12.

\section{O|livro|Apresentando_Rembrandt_-_Pintor,_Gravador,_Professor| .}

(The book Introducing Rembrandt - Painter, Recorder, Teacher.)

This is another important issue while training the Tokenizer component. By simply dividing at the underscore marks, we would train the tokenizer with tokens like Pintor, and it would conclude that sometimes a word followed by a comma can form a token, which is not true.

To avoid this, all these named entities were forcedly divided not only in the underscore, but also in all occasional punctuation marks.

\section{Evaluation}

The possible tokenizer configuration is:

- An algorithm (MAXENT or PERCEPTRON)

- A corpus - Floresta Virgem CETENFolha (VCF), Bosque CETENFolha (CF)

- Whether to include or not the abbreviation dictionary (ABB)

- Whether to use or not alphanumeric optimization (AO)

It was not possible to evaluate using the Amazônia corpus. Due to its size it required lots of memory, and even increasing the available memory, the evaluation was taking so long that it was unfeasible to wait any longer.

The experiments were divided into two parts. The first without the alphanumeric optimization and the second activating it. Each configuration was combined and evaluated for different cutoff values. 


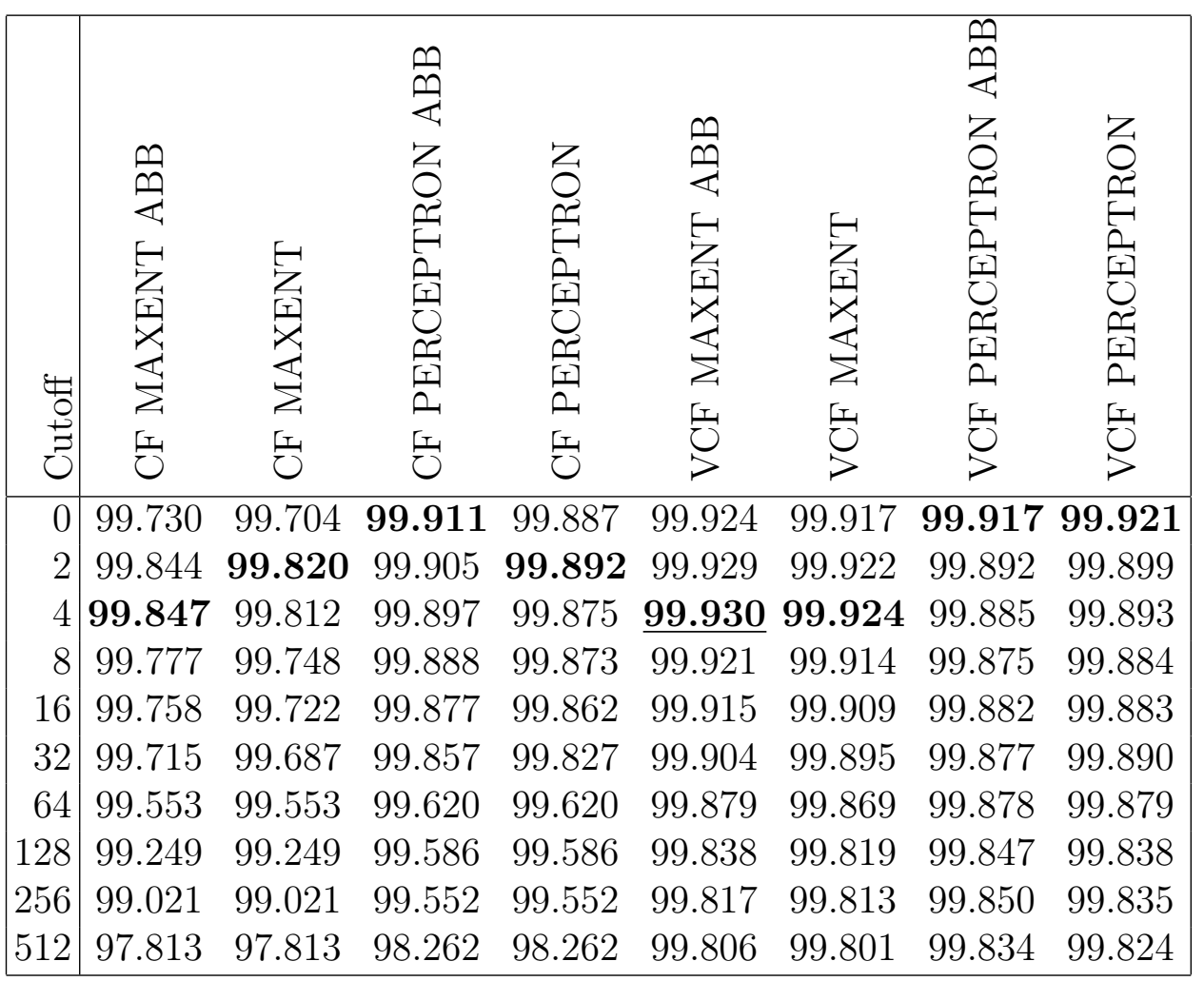

Table 6.9: 10-fold cross-validation results for different configurations of the tokenizer, with alphanumeric optimization off. The highest $F_{1}$ score of each configuration is in bold, and the overall highest score is underlined.

No alphanumeric optimization Table 6.9 summarizes the effectiveness results for models trained without alphanumeric optimization.

The highest $F_{1}$ score was $99.93 \%$ obtained by a Maxent model trained with VCF, cutoff three, using the abbreviation dictionary. Figure 6.3 shows how the $F_{1}$ scores are distributed according to the configuration and the cutoff.

It is clear that the corpus size influences the $F_{1}$ score, but the difference is not as important as it was for the sentence boundary detection. According to these experiments the maximum difference is $0.1 \%$, while for the sentence boundary detection, it surpasses $2 \%$.

Maxent models trained with VCF were better than Perceptron models trained with the same corpus, but the opposite occurred for CF, in which Perceptron models surpassed Maxent models trained with CF corpus. This shows that the Maxent algorithm works better with larger amounts of data in the tokenization task.

The impact of including the abbreviation dictionary was fairly higher for models trained with the smaller corpus. For models trained with the larger corpus, the abbreviation dictionary impacted positively only for Maxent models.

According to the curve shape, Perceptron models benefit from including all features, presenting a higher $F_{1}$ score at zero cutoff. Maxent models suffer from zero cutoff and perform better by increasing it. The effectiveness of models trained with the smaller corpus rapidly declines by increasing cutoff to values higher than four. Models trained with VCP 

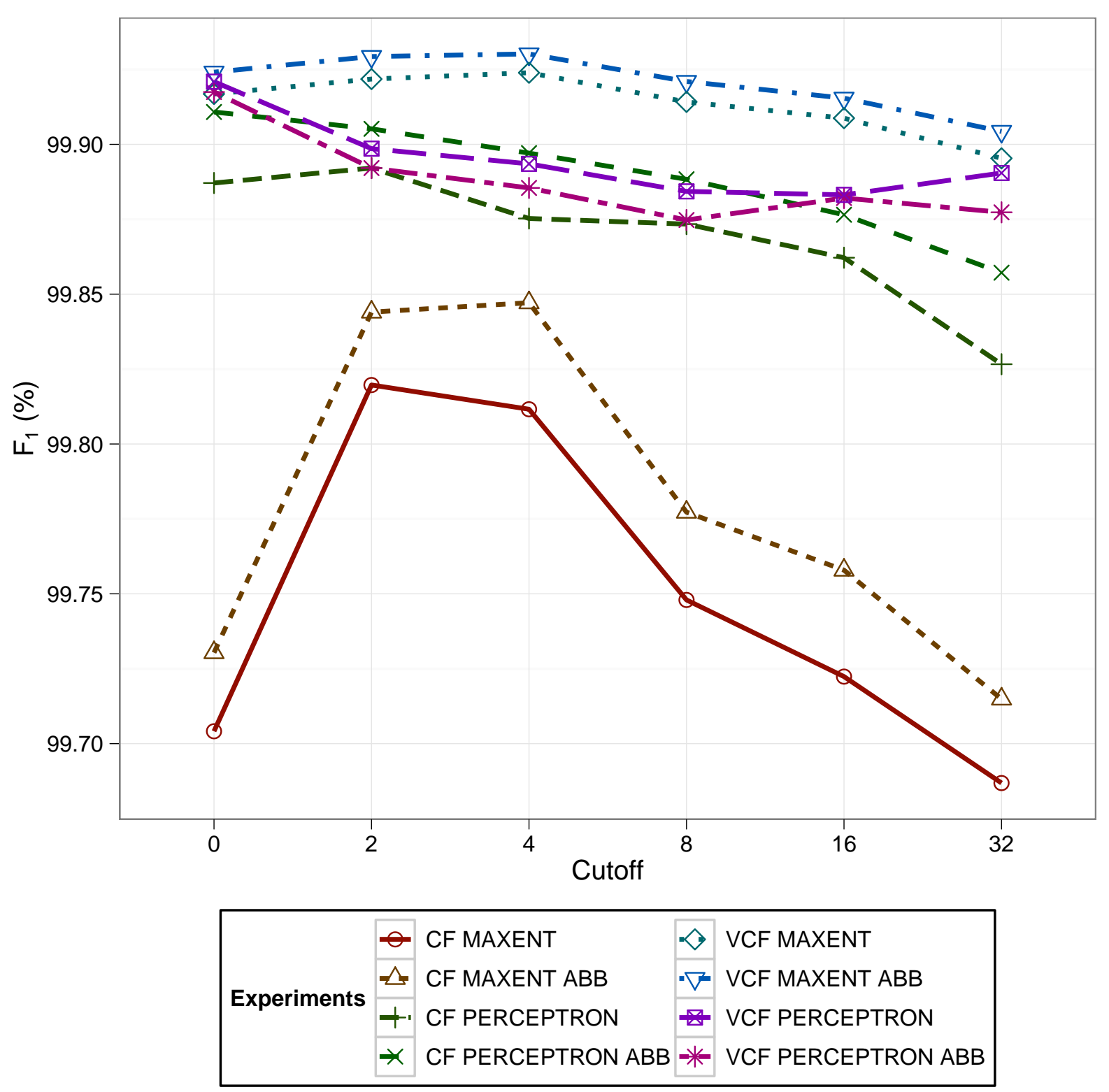

Figure 6.3: Distribution of the $F_{1}$ score according to the tokenizer configuration and the cutoff. $F_{1}$ values lower than $99.65 \%$ were not plotted.

appear to decline slower.

Table 6.10 illustrates an issue with Maxent models: their size. For any Maxent experiment the model size is larger compared to the equivalent Perceptron by orders of magnitude, surpassing megabytes while trained with VCF and kilobytes while trained with CF.

Alphanumeric optimization The following experiment measures the impact of alphanumeric optimization. The results are shown side-by-side compared to the equivalent experiment without alphanumeric optimization. Table 6.11 shows the impact in terms of $F_{1}$ score for the evaluations using CF corpus. The values barely changed, results with alphanumeric optimization being $0.01 \%$ lower than the corresponding ones without it. 


\begin{tabular}{|c|r|r|}
\hline Experiment & $F_{1}(\%)$ & Model Size $(\mathrm{kB})$ \\
\hline VCF MAXENT ABB & 99.930 & 1297 \\
VCF MAXENT & 99.924 & 1295 \\
VCF PERCEPTRON & 99.921 & 21 \\
VCF PERCEPTRON ABB & 99.917 & 22 \\
CF PERCEPTRON ABB & 99.911 & 9 \\
CF PERCEPTRON & 99.892 & 6 \\
CF MAXENT ABB & 99.847 & 245 \\
CF MAXENT & 99.820 & 473 \\
\hline
\end{tabular}

Table 6.10: Size of the best tokenizer model of each experiment. Lines are sorted by $F_{1}$

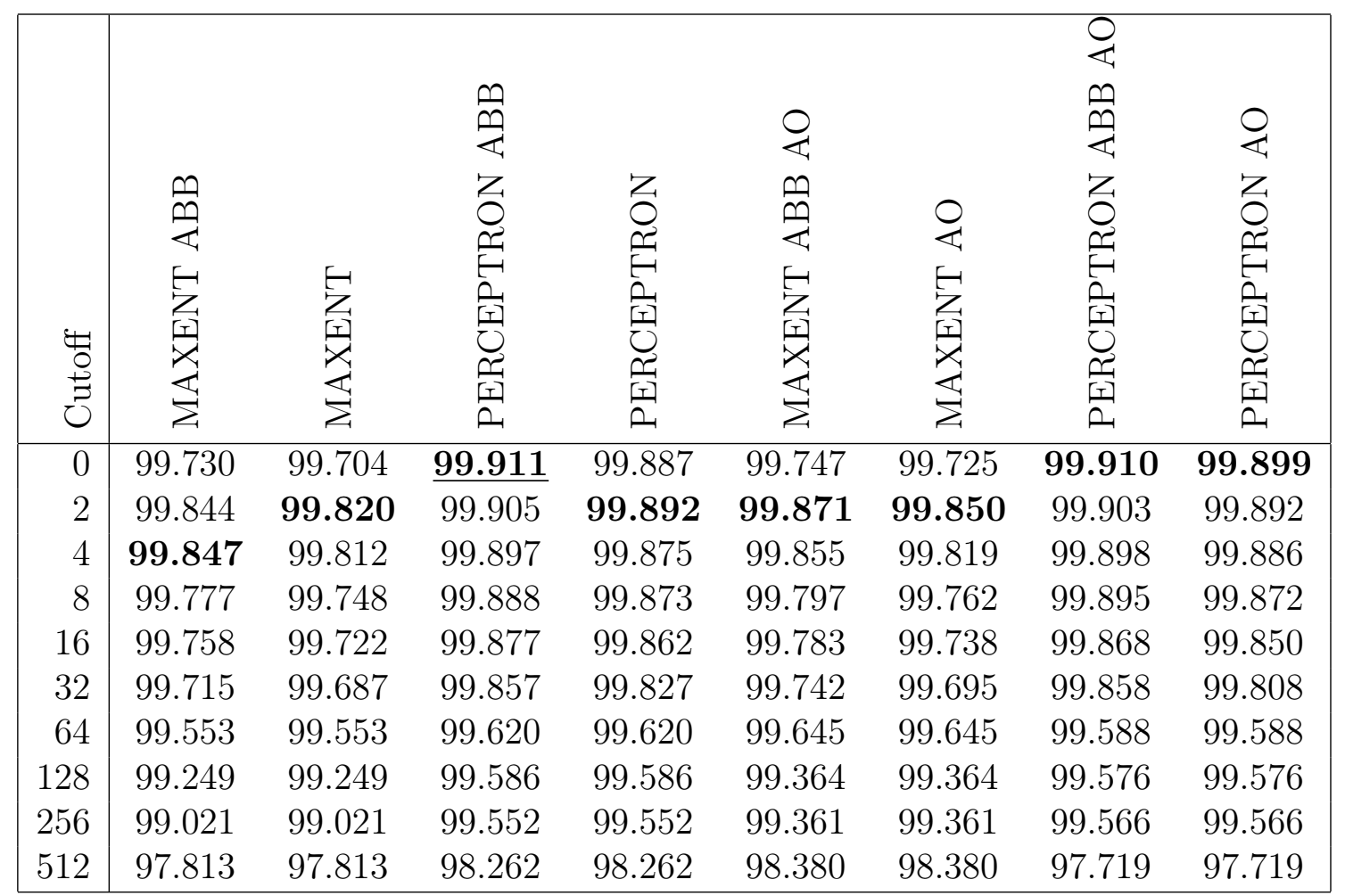

Table 6.11: 10-fold cross-validation results for different configurations of a tokenizer model trained with $C F$, including alphanumeric optimization.

Table 6.12 shows the results for the evaluations using VCF corpus. The alphanumeric optimization impact was the reverse of those from CF corpus. Again the values barely changed, but now results with alphanumeric are higher. For the Maxent algorithm the change is smaller than $0.01 \%$, but for Perceptron algorithm, the change is of $0.1 \%$.

In terms of model size, the alphanumeric optimization caused greater impact, at least for Maxent models. Table 6.13 shows the experiments with CF corpus. Maxent models trained without alphanumeric optimization are up to $100 k B$ larger than those with this option. The changes in Perceptron model are negligible, lower than $2 k B$.

Table 6.14 presents the same experiment results for VCF corpus. Again Maxent models benefit from alphanumeric optimization. Models trained without this option are $1 M B$ larger 


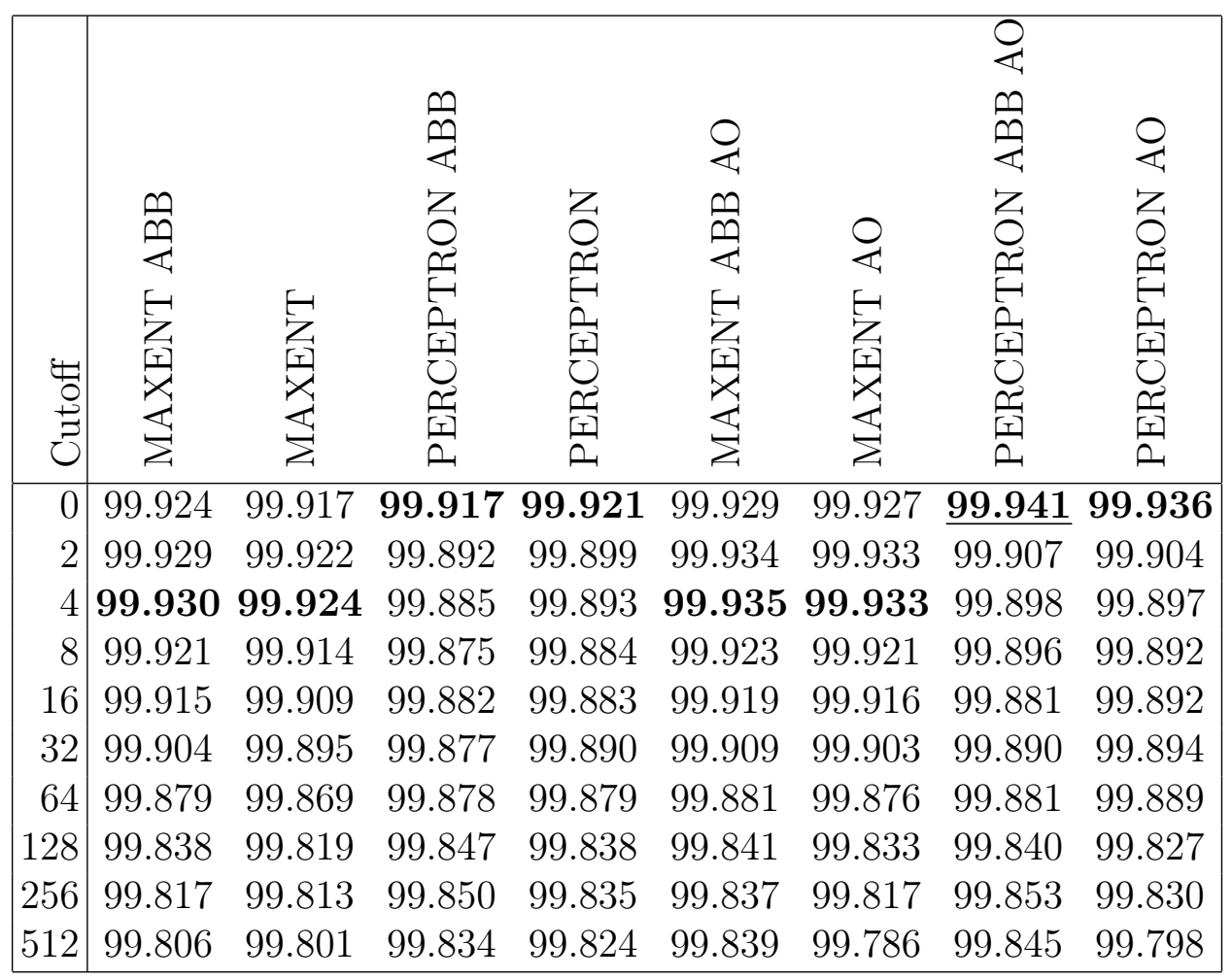

Table 6.12: 10-fold cross-validation results for different configurations of tokenizer model trained with VCF, including alphanumeric optimization.

\begin{tabular}{|c|r|r|}
\hline Experiment & $F_{1}(\%)$ & Model Size $(\mathrm{kB})$ \\
\hline PERCEPTRON ABB & 99.911 & 9 \\
PERCEPTRON ABB AO & 99.910 & 9 \\
PERCEPTRON AO & 99.899 & 8 \\
PERCEPTRON & 99.892 & 6 \\
MAXENT ABB AO & 99.871 & 220 \\
MAXENT AO & 99.850 & 218 \\
MAXENT ABB & 99.847 & 245 \\
MAXENT & 99.820 & 473 \\
\hline
\end{tabular}

Table 6.13: Size of the best tokenizer model of each experiment with CF corpus.

than its counterpart. The changes in Perceptron model are negligible, lower than $2 k B$.

\subsubsection{Previous Work}

The best tokenizer model proposed here scored 99.94\%, less than the rule based tokenizer described by (Silva, 2007), which scored $99.99 \%$ of $F_{1}$ over a $260 \mathrm{k}$-token corpus. In addition to the rule based technology, which allows fine-tuning the algorithm to the orthographic conventions of the language, this high score can be explained by its two-stage approach that counts with a POS tagger to solve ambiguities (Silva, 2007, p. 84).

On the other hand the best model scored significantly better than the tokenizer imple- 


\begin{tabular}{|c|r|r|}
\hline Experiment & $F_{1}(\%)$ & Model Size $(\mathrm{kB})$ \\
\hline PERCEPTRON ABB AO & 99.941 & 23 \\
PERCEPTRON AO & 99.936 & 25 \\
MAXENT ABB AO & 99.935 & 669 \\
MAXENT AO & 99.933 & 668 \\
MAXENT ABB & 99.930 & 1297 \\
MAXENT & 99.924 & 1295 \\
PERCEPTRON & 99.921 & 21 \\
PERCEPTRON ABB & 99.917 & 22 \\
\hline
\end{tabular}

Table 6.14: Size of the best tokenizer model of each experiment with VCF corpus.

mented by CoGrOO 3.1.2, whose $F_{1}$ score is, according to Section 3.2, $96.4 \%$.

\subsubsection{Evaluating the Grammar Checker}

This evaluation consists of replacing the tokenizer module of BASELINE by those proposed here and verifying the grammar checker effectiveness using annotated corpora. BASELINE is the CoGrOO 3.1.2 with the new sentence boundary detector proposed in Section 6.2 .

Because of the change in the hyphenated word handling described in Section 6.3.1 it was expected that the effectiveness of the grammar checker while handling sentences with hyphenated words would decrease. That proved to be true and most of the introduced false positives are related to this change, but it is expected that a new POS Tagger trained with separated hyphenated words will solve the issue.

The results are summarized in Table 6.15. The following sections presents the results.

\section{Metro Corpus}

No new true positives were found by introducing a new tokenizer, but the number of false positives varies drastically according to the configuration: some introduced one new false positive, others up to eight. Some configurations removed two false positives, others one or none.

The configuration that presented the best $F_{1}$ was VCF PERCEPTRON ABB AO. This configuration removed two false positives.

Example 6.13 shows one removed false positive related to hyphenated words. The $B A S E$ LINE did not separate the word know-how and later in the pipeline it was classified as a plural determiner, causing the false positive. With the new configuration the hyphenated 


\begin{tabular}{|c|c|c|c|c|c|c|c|}
\hline & \multicolumn{3}{|c|}{ Metro } & \multicolumn{3}{|c|}{ Probi } & Bosque \\
\hline Experiment & Target & $\mathrm{TP}$ & $\mathrm{FP}$ & Target & $\mathrm{TP}$ & $\mathrm{FP}$ & $\mathrm{FP}$ \\
\hline "BASELINE & & 16 & 34 & & 333 & 588 & 249 \\
\hline VCF PERCEPTRON ABB AO 0 & & 16 & $\begin{array}{r}33 \\
+1 \\
-2\end{array}$ & & $\begin{array}{r}334 \\
+1\end{array}$ & $\begin{array}{r}606 \\
+22 \\
-5\end{array}$ & $\begin{array}{r}252 \\
+14 \\
-11\end{array}$ \\
\hline VCF PERCEPTRON AO 0 & & 16 & $\begin{array}{r}33 \\
+1 \\
-2\end{array}$ & & $\begin{array}{r}334 \\
+1\end{array}$ & $\begin{array}{r}606 \\
+22 \\
-5\end{array}$ & $\begin{array}{r}253 \\
+15 \\
-11\end{array}$ \\
\hline $\begin{array}{l}\text { VCF MAXENT ABB AO } 4 \\
\text { VCF MAXENT AO } 4\end{array}$ & & 16 & $\begin{array}{r}34 \\
+1 \\
-1\end{array}$ & & $\begin{array}{c}334 \\
+1\end{array}$ & $\begin{array}{r}607 \\
+23 \\
-5\end{array}$ & $\begin{array}{r}251 \\
+15 \\
-13\end{array}$ \\
\hline VCF MAXENT ABB 4 & & 16 & $\begin{array}{r}34 \\
+1 \\
-1\end{array}$ & & $\begin{array}{r}334 \\
+1\end{array}$ & $\begin{array}{r}606 \\
+22 \\
-5\end{array}$ & $\begin{array}{r}252 \\
+12 \\
-9\end{array}$ \\
\hline VCF MAXENT 4 & & 16 & $\begin{array}{r}34 \\
+1 \\
-1\end{array}$ & & $\begin{array}{c}334 \\
+1\end{array}$ & $\begin{array}{r}607 \\
+22 \\
-4\end{array}$ & $\begin{array}{r}252 \\
+12 \\
-9\end{array}$ \\
\hline VCF PERCEPTRON 0 & & 16 & $\begin{array}{r}34 \\
+2 \\
-2\end{array}$ & & $\begin{array}{r}334 \\
+1\end{array}$ & $\begin{array}{r}606 \\
+22 \\
-5\end{array}$ & $\begin{array}{r}254 \\
+16 \\
-11\end{array}$ \\
\hline VCF PERCEPTRON ABB 0 & 53 & 16 & $\begin{array}{r}33 \\
+1 \\
-2\end{array}$ & 2616 & $\begin{array}{c}334 \\
+1\end{array}$ & $\begin{array}{r}606 \\
+22 \\
-5\end{array}$ & $\begin{array}{r}252 \\
+15 \\
-12\end{array}$ \\
\hline CF PERCEPTRON ABB 0 & & 16 & $\begin{array}{r}38 \\
+6 \\
-2\end{array}$ & & $\begin{array}{r}334 \\
+1\end{array}$ & $\begin{array}{r}606 \\
+22 \\
-5\end{array}$ & $\begin{array}{r}277 \\
+44 \\
-16\end{array}$ \\
\hline CF PERCEPTRON ABB AO 0 & & 16 & $\begin{array}{r}41 \\
+8 \\
-1\end{array}$ & & $\begin{array}{r}334 \\
+1\end{array}$ & $\begin{array}{r}606 \\
+22 \\
-5\end{array}$ & $\begin{array}{r}277 \\
+44 \\
-16\end{array}$ \\
\hline CF PERCEPTRON AO 0 & & 16 & $\begin{array}{r}39 \\
+7 \\
-2\end{array}$ & & $\begin{array}{r}334 \\
+1\end{array}$ & $\begin{array}{r}607 \\
+22 \\
-4\end{array}$ & $\begin{array}{r}277 \\
+44 \\
-16\end{array}$ \\
\hline CF PERCEPTRON 2 & & 16 & $\begin{array}{r}39 \\
+6 \\
-1\end{array}$ & & $\begin{array}{r}334 \\
+1\end{array}$ & $\begin{array}{r}607 \\
+22 \\
-4\end{array}$ & $\begin{array}{r}277 \\
+44 \\
-16\end{array}$ \\
\hline $\begin{array}{l}\text { CF MAXENT ABB AO } 2 \\
\text { CF MAXENT AO } 2\end{array}$ & & 16 & $\begin{array}{r}36 \\
+3 \\
-1\end{array}$ & & $\begin{array}{c}334 \\
+1\end{array}$ & $\begin{array}{r}610 \\
+27 \\
-6\end{array}$ & $\begin{array}{r}276 \\
+44 \\
-17\end{array}$ \\
\hline $\begin{array}{l}\text { CF MAXENT ABB } 4 \\
\text { CF MAXENT } 4\end{array}$ & & 16 & $\begin{array}{r}37 \\
+3\end{array}$ & & $\begin{array}{c}334 \\
+1\end{array}$ & $\begin{array}{r}612 \\
+27 \\
-4\end{array}$ & $\begin{array}{r}277 \\
+45 \\
-17 \\
\end{array}$ \\
\hline
\end{tabular}

Table 6.15: Summary of the grammar checker evaluation using different configuration of the tokenizer module. The number in the experiment name refers to the cutoff value used. Bosque corpus does not include grammar error annotations. Signed numbers refer to removed (when negative) or added (when positive) entries. 
words was tokenized and the group was classified as noun, removing the false positive.

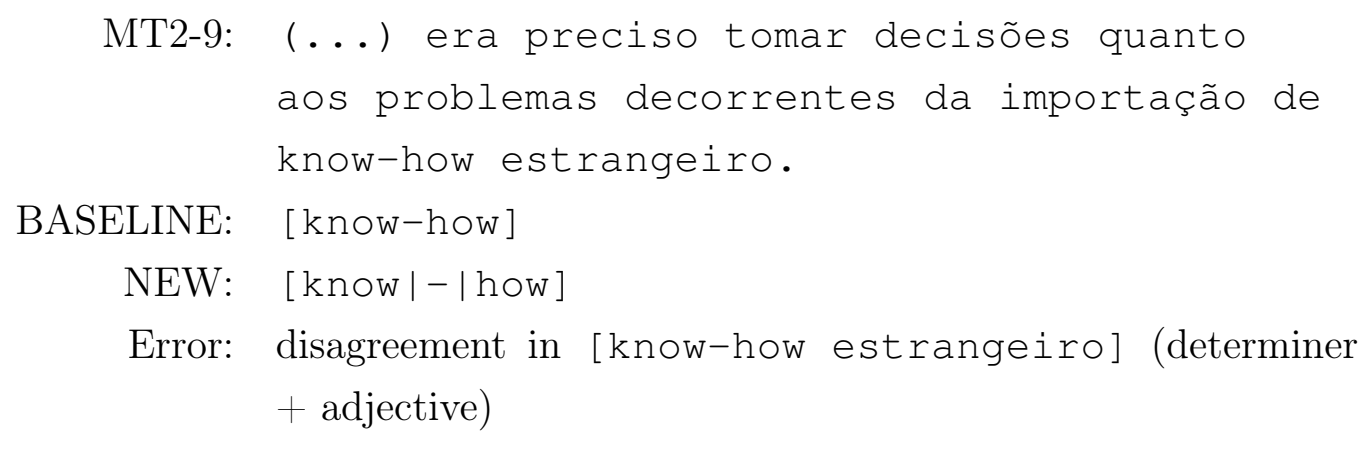

Example 6.14 is related to punctuation. The text author missed a space between the word Verde and the opening parenthesis. BASELINE did not separate this into two tokens, and later in the pipeline it was wrongly classified, causing a false positive. The new configuration was separated into two tokens and because of it the correct class was associated with each token, removing the false positive.

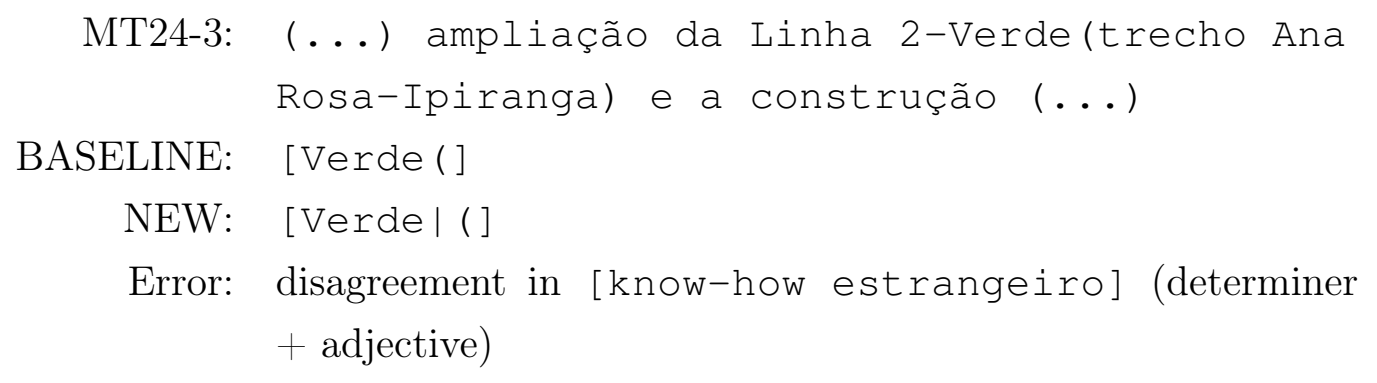

The false positive introduced by VCF PERCEPTRON ABB AO was related to the hyphenated word e-mail, and improvements in this are expected with the improvements in the POS tagger.

These results were similar to all models trained with VCF, except for the issue described in 6.14, that was only reproduced by models trained with Perceptron algorithm.

Models trained with CF caused more false positives, all related to the token 4-Lilás from the phrase Linha 4-Lilás, which occurs four times in the corpus. The models trained with CF corpus split it in $4|-|$ Lilás, while ones trained with VCF kept it as one token. In the first case the name finder components classified Linha 4 as a proper name, and in the latter case, it classified Linha 4-Lilás as a proper name instead. This will probably improve with a new trained name finder and POS tagger.

\section{Probi Corpus}

One new true positive, Example 6.15, was included with all configurations evaluated with Probi corpus. By splitting the token padre-diretor, its number feature changed to 
singular, triggering the true positive.

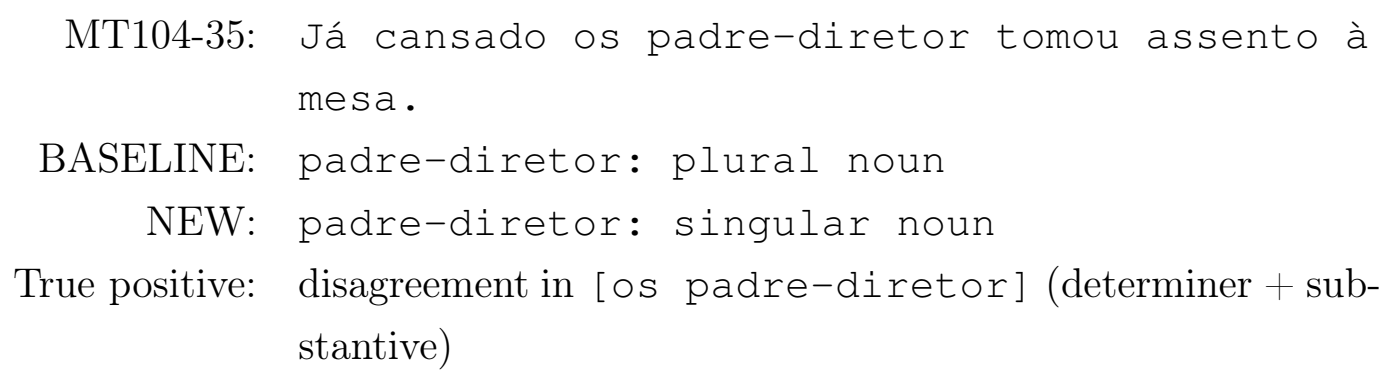

Some configurations removed four false positives, others five or six.

A curious case is shown in Example 6.16. By splitting the abbreviation Dr. in two tokens, the inappropriate final mark in the sentence caused some misclassification later in the pipeline, causing a false positive. Perceptron models trained with VCF correctly kept Dr. as one token, but for Maxent models trained with VCF, only configurations that include the alphanumeric optimization or abbreviation dictionary did so. Models trained with CF, only the Perceptron model that included an abbreviation dictionary kept the Dr. as one token.

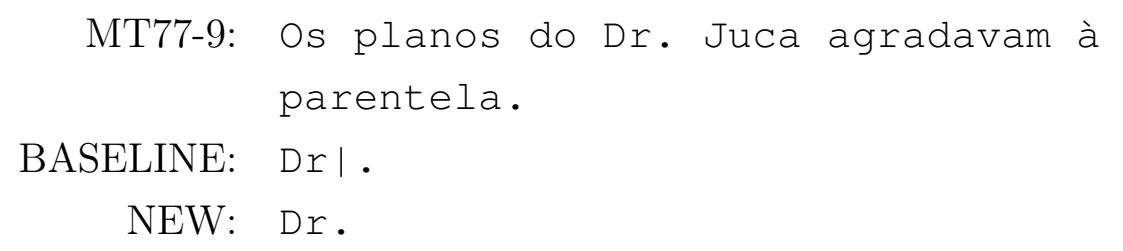

All the other changes in false positives are related to the changes in hyphenated words.

\section{Bosque Corpus}

Evaluation with the Bosque corpus showed improvements while dealing with numbers with thousands or decimal separators, which in Portuguese are dot and comma respectively. BASELINE frequently splits numbers like 2,43 or 1.500 in two tokens, and often this causes false positives. All new tokenizer configurations would keep numbers as one token. For the VCF PERCEPTRON ABB AO, it means four out of eleven removed false positives, and 5 out of 14 introduced false positives.

The introduced false positives related to numbers with thousands or decimal separators can be explained by the fact that the BASELINE POS tagger, as example of the BASELINE tokenizer, also can not deal with these numbers. 6.17 exemplifies one of the issues. The $B A S E L I N E$ tokenizer split the number in two and eventually erroneously classified the token , 8 as a determiner, but as all the other tokens were also classified as singular, it did not cause a false positive. The new tokenizer kept 1,8 as one token, but it was erroneously 
classified as plural. This issue is expected to be addressed with the later improvements.

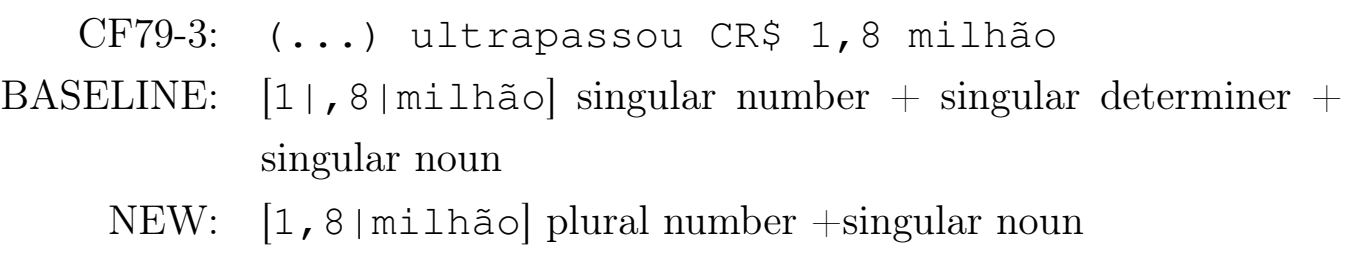

Besides the issue with the numbers, there was also significant issues with quotation marks in this corpus. Differing from Metro and Probi, where quotation marks were neutral, in Probi they are oriented (opening and closing) and represented by the symbols « and ». Although this symbol is used while training the tokenizer, occasionally characters like «traveler is maintained as one token.

Four out of the fourteen false positives introduced by VCF PERCEPTRON ABB AO were related to this issue with quotation marks. Models trained with $\mathrm{CF}$ showed even worse results, and up to thirty out of forty-five new false positives was related to this issue.

Most of the other new false positives introduced are related to hyphenated words.

\subsubsection{Conclusion}

Although the new Tokenizer did not improve the grammar checker evaluation scores, the output of the module improved, especially concerning numbers and punctuation. Due to this, when choosing the best configuration, only the 10-fold cross-validation scores of the module were taken into account, and the best effective configuration was:

- Algorithm: Perceptron

- Abbreviation dictionary: yes

- Alphanumeric optimization: yes

- Corpus: Virgem CETEM Folha

- Cutoff: zero 


\subsection{Name Finder}

The Brazilian Portuguese corpora used for training (CETENFolha, Virgem CETENFolha and Amazonia) arranges proper nouns into just one token. Due to this characteristic, before annotating the text using a part-of-speech tagger trained with these corpora, one has to preprocess its tokens by grouping proper names.

The name finder module is based on the Apache OpenNLP module with the same name. The proposed improvement is to train and evaluate using the latest version of this library.

The adapted Apache OpenNLP Name Finder algorithm works as follows:

- The module receives the tokens of a sentence;

- Each token is a candidate to start a proper name, or to continue one;

- Features are collected from the context and are used to decide if it is a proper name or not;

- The tokens of the identified names are merged as one token.

To train the module the named entities were extracted from the corpus using the following criteria: a) single token annotated as proper name; b) tokens grouped with underscores annotated as compound proper name.

There is a difference between Floresta Virgem CETENFolha (VCF) and Bosque CETENFolha (CF). As in Example 6.18, in VCF the monetary entities are grouped and annotated as proper name, while in CF they are represented as multiple tokens. It can cause important divergences in the evaluation, and also it impacts which corpus is used to train the part-of-speech tagger.

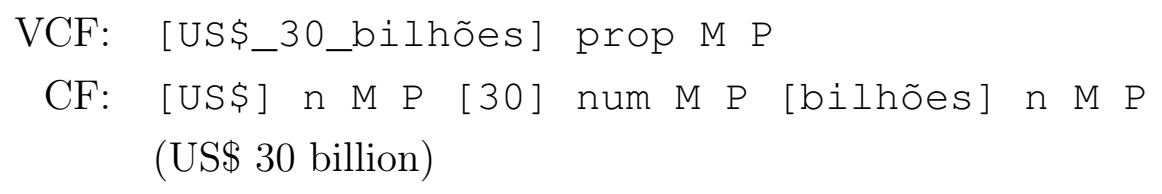

\subsubsection{Default Contextual Predicates}

Lets define Candidate as the token been analyzed, and Index the position of the candidate in the sentence.

The contextual templates have the following form:

- For each token in a window of size 5, take the token lexeme, the token class and the token class concatenated with its lexeme;

- The concatenation of the current token and the previous token;

- The concatenation of the current token class and the previous token class; 
- Whether the current token starts a sentence;

- An outcome selected for the previous token.

The token classes, ordered by priority, are: 1) lowercase alphabetic; 2) two digits; 3) four digits; 4) alpha-numeric; 5) digits and dashes; 6) digits and slashes; 7) digits and commas; 8) digits and periods; 9) digits; 10) single capital letter; 11) initial capital letter; 12) other.

\section{Evaluation}

The possible name finder configuration are:

- An algorithm (MAXENT or PERCEPTRON)

- A corpus - Floresta Virgem CETENFolha (VCF), Bosque CETENFolha (CF)

It was not possible to evaluate using the Amazônia corpus. Due to its size, it required a great deal of memory, and even increasing the available memory, the evaluation was taking so long that it was unfeasible to wait any longer.

Table 6.16 summarizes the effectiveness results for Floresta Virgem CETENFolha and Bosque CETENFolha.

\begin{tabular}{|c|c|c|c|c|}
\hline 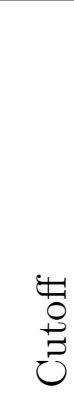 & 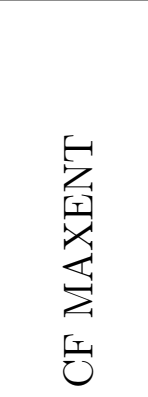 & 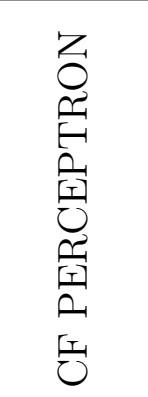 & 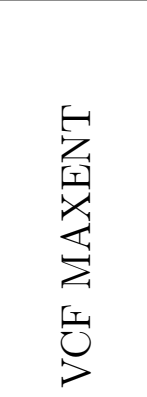 & 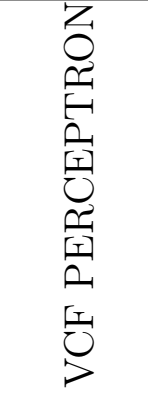 \\
\hline 0 & 81.925 & 87.484 & 85.117 & $\underline{88.402}$ \\
\hline 2 & 84.891 & 85.932 & 86.256 & 86.807 \\
\hline 4 & 85.935 & 83.470 & 86.655 & 84.956 \\
\hline 8 & 85.913 & 85.342 & 86.647 & 83.805 \\
\hline 16 & 84.376 & 85.935 & 86.640 & 83.656 \\
\hline 32 & 83.757 & 85.007 & 86.570 & 76.642 \\
\hline 64 & 82.514 & 83.600 & 86.267 & 74.913 \\
\hline 128 & 81.881 & 82.013 & 85.441 & 76.296 \\
\hline 256 & 81.073 & 81.061 & 84.314 & 75.958 \\
\hline 512 & 80.511 & 79.223 & 83.373 & 74.168 \\
\hline
\end{tabular}

Table 6.16: 10-fold cross-validation results for different configurations of the name finder. The highest $F_{1}$ score of each configuration is in bold, and the overall highest score is underlined.

The highest $F_{1}$ score was $88.402 \%$ obtained by a Perceptron model trained with VCF, cutoff zero. Figure 6.4 shows how the $F_{1}$ scores are distributed according to the configuration and the cutoff. 


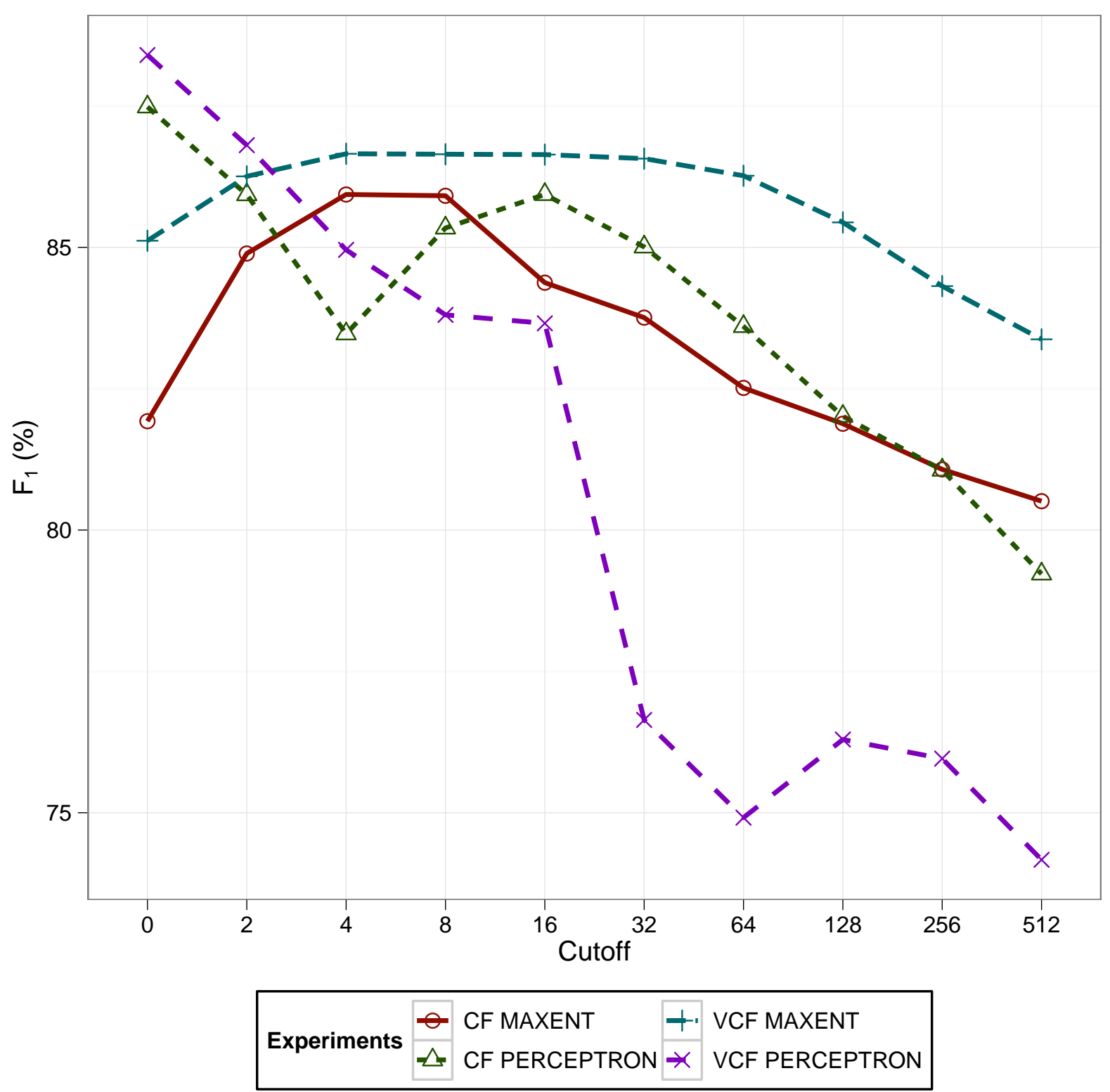

Figure 6.4: Distribution of the $F_{1}$ score according to the name finder configuration and the cutoff.

The corpus size influences the $F_{1}$ score, models trained with VCF corpus are around $1 \%$ more effective in terms of $F_{1}$ compared to CF corpus. Independent of the corpus used, Perceptron models surpass Maxent models by around $2 \%$.

According to the curve shape, Perceptron models benefit from including all features, presenting a higher $F_{1}$ score at zero cutoff. Maxent models suffer from zero cutoff and perform better by increasing it. The effectiveness of models trained with the smaller corpus rapidly declines by increasing cutoff to values higher than eight. Models trained with VCP appear to decline slower. The $F_{1}$ score of models trained with Perceptron declines abruptly for cutoff values higher than 16 .

The model size, as shown in Table 6.17, was influenced by the corpus and algorithm. The Perceptron model trained with VCF is fairly larger than the model trained with CF, 
the same algorithm. If the grammar checker evaluation shows similar effectiveness results, the smaller should be used.

\begin{tabular}{|c|r|r|}
\hline Experiment & $F_{1}(\%)$ & Model Size $(\mathrm{kB})$ \\
\hline VCF PERCEPTRON & 88.402 & 754 \\
CF PERCEPTRON & 87.484 & 83 \\
VCF MAXENT & 86.655 & 3854 \\
CF MAXENT & 85.935 & 401 \\
\hline
\end{tabular}

Table 6.17: Size of the best name finder model of each experiment. Lines are sorted by $F_{1}$

\subsubsection{Previous Work}

Named entity recognition is one of the most explored Natural Language Processing tasks. Seidel (2012) recently developed a mentioned entity recognition tool for Portuguese using the Maxent framework. The tool cannot only recognize a number of entities, but also classify them according to its type, like person, place, company and others. For the identification task, they reported a $F_{1}$ score of $73.68 \%$. The evaluation was performed with a specific corpus, and it is not possible to directly compare its results with those obtained by the new Proper Name Finder module proposed here.

\subsubsection{Evaluating the Grammar Checker}

This evaluation consists of replacing the name finder module of BASELINE by those proposed here and verifying the grammar checker effectiveness using annotated corpora. BASELINE is the CoGrOO 3.1.2 with the new sentence boundary detector and tokenizer proposed in Sections 6.2 and 6.3.

The results are summarized in Table 6.18. The following sections present the results.

\section{Metro Corpus}

No new true positives were found by introducing a new name finder. The number of false positives did not change, but that is a coincidence because the same number of false positives were introduced and removed.

The configuration that presented the best $F_{1}$ in the 10 -fold cross-validation was $V C F$ PERCEPTRON. At the grammar checker evaluation it removed four false positives and added another four occurrences.

Example 6.19 shows one removed false positive related to monetary expressions, that are grouped as one token in VCF. The false positive was caused by the discrepancy between US\$_922 and milhões because the POS Tagger erroneously classified the first token as a singular number. The new name finder grouped [US\$_922_milhões] as one token, 


\begin{tabular}{|c|c|c|c|c|c|c|c|}
\hline & \multicolumn{3}{|c|}{ Metro } & \multicolumn{3}{|c|}{ Probi } & Bosque \\
\hline Experiment & Target & $\mathrm{TP}$ & FP & Target & $\mathrm{TP}$ & $\mathrm{FP}$ & $\mathrm{FP}$ \\
\hline BASELINE & & 16 & 33 & & 334 & 606 & 252 \\
\hline VCF PERCEPTRON 0 & & 16 & $\begin{array}{r}33 \\
+4 \\
-4\end{array}$ & & $\begin{array}{r}328 \\
+1 \\
-7\end{array}$ & $\begin{array}{c}603 \\
+7 \\
-10\end{array}$ & $\begin{array}{r}231 \\
+21 \\
-42\end{array}$ \\
\hline CF PERCEPTRON 0 & 53 & 16 & $\begin{array}{r}33 \\
+5 \\
-5\end{array}$ & 2616 & $\begin{array}{r}328 \\
+1 \\
-7\end{array}$ & $\begin{array}{r}605 \\
+8 \\
-9\end{array}$ & $\begin{array}{r}243 \\
+18 \\
-27\end{array}$ \\
\hline VCF MAXENT 4 & & 16 & $\begin{array}{r}33 \\
+4 \\
-4\end{array}$ & & $\begin{array}{r}328 \\
+1 \\
-7\end{array}$ & $\begin{array}{r}603 \\
+6 \\
-9\end{array}$ & $\begin{array}{r}230 \\
+21 \\
-43\end{array}$ \\
\hline CF MAXENT 4 & & 16 & $\begin{array}{r}33 \\
+5 \\
-5\end{array}$ & & $\begin{array}{r}329 \\
+1 \\
-6\end{array}$ & $\begin{array}{r}600 \\
+6 \\
-12\end{array}$ & $\begin{array}{r}242 \\
+18 \\
-28\end{array}$ \\
\hline
\end{tabular}

Table 6.18: Summary of the grammar checker evaluation using different configuration of the proper name finder module. The number in the experiment name refers to the cutoff value used. Bosque corpus does not include grammar error annotations.

avoiding the misclassification. The name finder helped here, but the real issue is in the POS tagger.

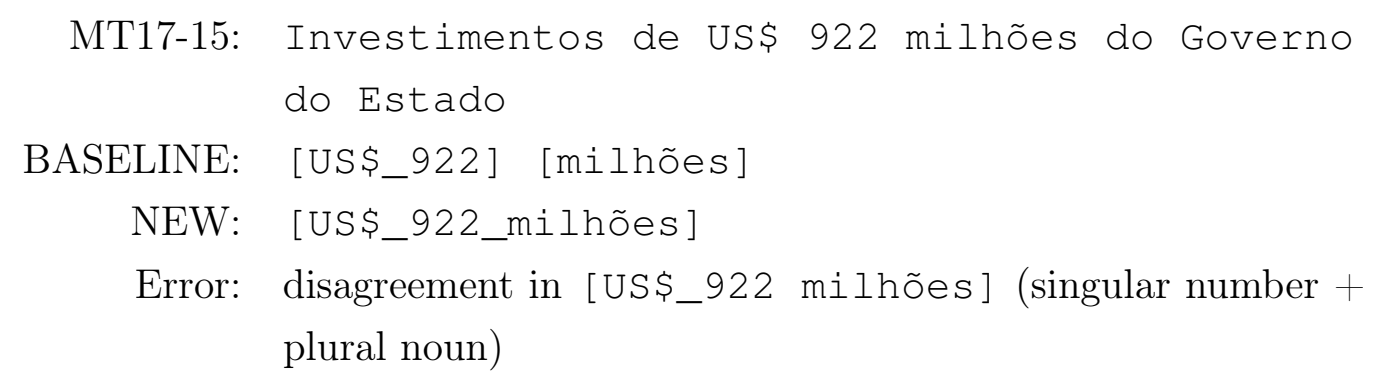

Example 6.20 is another removed false positive. The BASELINE erroneously classified biblioteca da estação as a feminine, singular proper name, followed by the masculine, singular proper name Paraíso. This arrangement caused an error. The other two removed false positives are similar to this example.

MT33-1: A biblioteca da estação Paraíso conta (...)

BASELINE: [A] [biblioteca_da_estação] [Paraíso]

NEW: [A] [biblioteca] [da] [estação] [Paraíso]

Error: [biblioteca_da_estação] classified grouped as a name

The four or five introduced false positives are related to the token reorganization caused by proper names introduced or omitted by the new configuration, but frequently the changes 
are for the better. This reorganization caused different POS tags to be associated with each token, or even different chunks or subjects were found. It is expected that the new POS tagger, chunker and shallow parser will deal with these reorganizations better and remove the new false positives.

The model VCF MAXENT presented the same results. The difference in CF PERCEPTRON and CF MAXENT are related to token reorganization and to the fact that VCF groups monetary entities in one token, while CF does not, as explained in the beginning of this section.

\section{Probi Corpus and Bosque}

One new true positive, Example 6.21, was included by all configurations evaluated with Probi corpus. BASELINE did not group Minas Gerais, and was checking if Gerais agrees with insdustrializam. The new name finder groups the proper name correctly, and correctly identifies the error.

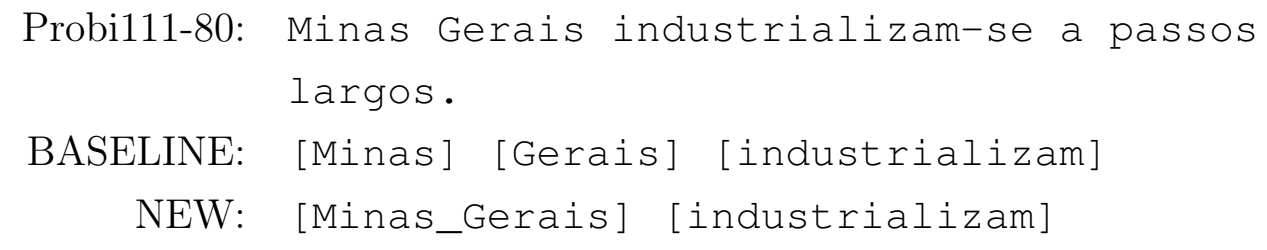

True positive: disagreement in [Minas_Gerais] [industrializam] (singular proper name + plural verb)

All configurations but the CF MAXENT removed one true positive, Example 6.22, because the error is inside the proper name. By correctly finding the name, the error was hidden when the tokens were grouped.

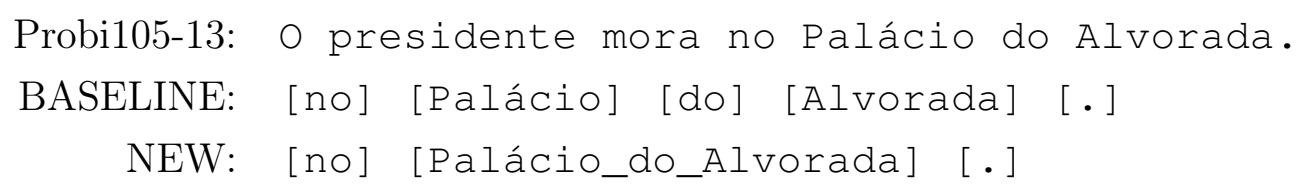

False negative: do (male) does not agree with Alvorada (feminine), but because it was grouped as a token the error is now hidden.

There are another six true positives that were removed by all configurations of the name finder. Similar to Example 6.22, it was caused by an error hidden by the grouped proper name. All the six errors are of a crase accent before treatment pronouns. The treatment pronoun was grouped together with the following proper name, hiding the existence of the 
error. See Example 6.23. To fix it a new rule would be added to check this case.

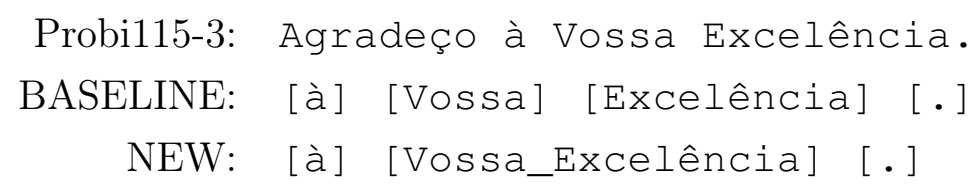

False negative: misuse of à before the treatment pronoun Vossa, but it got hidden when the name finder grouped it as one token.

Similar to what happened while evaluating the grammar checker with Metro corpus, many false negatives were removed and introduced while evaluating with Probi and Bosque. The changes were caused by differences in tokens that were grouped as proper name by $B A S E L I N E$ or by a new configuration, and this changes leads to a difference on how tokens were classified by POS tagger, chunker or shallow parser. By inspecting the changes it is clear that the new name finders made a better job finding names, and that is why more false positives were removed then introduced.

Models trained with VCF removed 12 false positives related to monetary expressions. It is due to the fact that this corpus also groups monetary expressions as a proper noun and it causes less errors while handling this kind of structure, as already exemplified in 6.19 for Metro corpus.

\subsubsection{Conclusion}

Although for some experiments, like the grammar checker experiment with Probi corpus, the new Proper Name Finder slightly degraded the effectiveness score, a deeper analysis showed that the module outputs more consistent values.

Considering the $F_{1}$ results in 10-fold cross-validation, the model size, and the grammar checker effectiveness. The best model is the one trained with Perceptron algorithm over Bosque CETENFolha corpus:

- Algorithm: Perceptron

- Corpus: Bosque CETENFolha

- Cutoff: zero 


\subsection{Contraction Finder}

The Contraction Finder module replaces the Post Tokenizer module from 3.1.2, described in Section 3.1.3. This module finds and expands Brazilian Portuguese contractions. This step is required because in the Brazilian Portuguese corpora used for training (CETENFolha, Virgem CETENFolha and Amazonia) all contractions are expanded.

Contractions are usually formed by a preposition with an article or pronoun. For example: da $(\mathrm{de}+\mathrm{a})$, em $(e m+$ ele $)$, etc.

CoGrOO 3.1.2 does a simple dictionary lookup to check if a token is a contraction. Table 6.19 shows the results of this approach. As expected, the recall is $100 \%$, but it is not that precise due to ambiguity. For example consigo can be the contraction of the preposition com and the pronoun sigo, or the present form of the verb conseguir.

\begin{tabular}{|l|c|c|c|}
\cline { 2 - 4 } \multicolumn{1}{c|}{} & Precision & Recall & $F_{1}$ \\
\hline CF & 99.594 & 1 & 99.797 \\
\hline VCF & 99.616 & 1 & 99.806 \\
\hline
\end{tabular}

Table 6.19: Evaluation results for the dictionary lookup approach.

The proposed improvement is to create a trainable component that would decide if the word is a contraction according to the context. The new component is adapted from Apache OpenNLP Name Finder.

The adapted Apache OpenNLP name finder algorithm works as follows:

- The module receives the tokens of a sentence;

- Each token is a contraction candidate;

- Features are collected from the context to decide if it is a contraction or not;

- The identified contraction is expanded into tokens using a dictionary.

\subsubsection{Contextual Predicates}

Preliminary experiments, which results are detailed in Table 6.20, demonstrated that the default contextual predicates of the Name Finder component of Apache OpenNLP, explained in Section 6.4.1, was not suitable to the task of finding contractions. The results were obtained by evaluating a Maxent model with cutoff 5 using Bosque CETENFolha corpus for three different contextual predicate configurations.

The effectiveness of the module trained using the default contextual predicates is quite poor. The precision was almost the same as performing a simple dictionary lookup, and recall was too low. The result could be improved with the introduction of a contraction dictionary feature, that checks whether a word is a known contraction or not. With this change the recall increased substantially, but precision increased only $0.04 \%$. 


\begin{tabular}{|l|c|c|c|}
\cline { 2 - 4 } \multicolumn{1}{c|}{} & Precision & Recall & $F_{1}$ \\
\hline Default contextual predicates & 99.589 & 94.259 & 96.851 \\
\hline Default + contraction dictionary & 99.630 & 99.852 & 99.741 \\
\hline Suffix (window) + contraction dictionary & 99.741 & 99.852 & 99.796 \\
\hline
\end{tabular}

Table 6.20: Evaluation results of the preliminary experiments.

Another experiment was to remove each of the default contextual predicates, one by one, to evaluate their impact. This proved that the default contextual predicates have no impact in this task because the precision and recall values did not change. So these contextual predicates were removed. The last experiment was to introduce a new contextual predicate based on the suffix of the previous two tokens, as well as of the following two tokens. This change increased precision by $0.152 \%$.

Lets define Candidate the token been analyzed. The resultant contextual templates have the following form:

- For the previous two tokens and following two tokens, get the suffixes;

- Check if the candidate is in the contraction dictionary.

\section{Evaluation}

The possible contraction finder configuration are:

- An algorithm (MAXENT or PERCEPTRON)

- A corpus - Floresta Virgem CETENFolha (VCF), Bosque CETENFolha (CF)

It was not possible to evaluate using the Amazônia corpus. Due to its size it required lots of memory, and even increasing the available memory, the evaluation was taking so long that it was unfeasible to wait any longer.

Table 6.21 summarizes the effectiveness results.

The highest $F_{1}$ score was $99.947 \%$ obtained by a Perceptron model trained with VCF, cutoff eight. Figure 6.5 shows how the $F_{1}$ scores are distributed according to the configuration and the cutoff.

The corpus size influences the $F_{1}$ score, models trained with VCF corpus are around $0.06 \%$ to $0.95 \%$ more effective in terms of $F_{1}$ compared to the CF corpus.

This is the first evaluated module that showed Perceptron models did not benefit from including all features, presenting higher $F_{1}$ scores occurred for higher cutoff values. The effectiveness of models do not decline drastically for higher cutoff values. This might be due to the fact that often contractions are not ambiguous, so the task is generally trivial.

The model size, as show in Table 6.22, was influenced by the chosen corpus and algorithm. The Perceptron model trained with the VCF corpus is small, but it has double the size of 


\begin{tabular}{|c|c|c|c|c|}
\hline 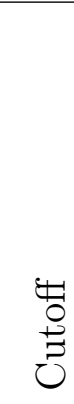 & 点 & 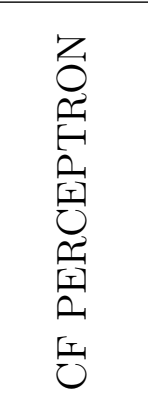 & 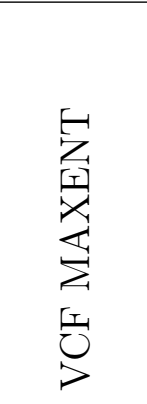 & 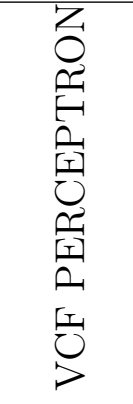 \\
\hline 0 & 99.806 & 99.834 & 99.881 & 99.937 \\
\hline 2 & 99.787 & 99.815 & 99.880 & 99.943 \\
\hline 4 & 99.796 & 99.824 & 99.882 & 99.945 \\
\hline 8 & 99.806 & 99.824 & 99.877 & 99.947 \\
\hline 16 & 99.787 & 99.815 & 99.874 & 99.945 \\
\hline 32 & 99.787 & 99.806 & 99.859 & 99.934 \\
\hline 64 & 99.824 & 99.824 & 99.852 & 99.940 \\
\hline 128 & 99.787 & 99.852 & 99.843 & 99.928 \\
\hline 256 & 99.778 & 99.806 & 99.841 & 99.925 \\
\hline 512 & 99.797 & 99.796 & 99.834 & 99.918 \\
\hline
\end{tabular}

Table 6.21: 10-fold cross-validation results for different configurations of the contraction finder. The highest $F_{1}$ score of each configuration is in bold, and the overall highest score is underlined.

the model trained with the CF corpus, the same algorithm. The Maxent model trained with CF corpus is much larger than the Perceptron model. The Maxent model trained with VCF corpus is one megabyte larger than the one trained with CF corpus.

\begin{tabular}{|c|r|r|}
\hline Experiment & $F_{1}(\%)$ & Model Size $(\mathrm{kB})$ \\
\hline VCF PERCEPTRON & 99.947 & 15 \\
VCF MAXENT & 99.882 & 1380 \\
CF PERCEPTRON & 99.852 & 7 \\
CF MAXENT & 99.824 & 369 \\
\hline
\end{tabular}

Table 6.22: Size of the best contraction finder model of each experiment. Lines are sorted by $F_{1}$

\subsubsection{Previous Work}

As of the date this document was written, there was no information on any specialized tool specifically for this task.

\subsubsection{Evaluating the Grammar Checker}

This evaluation consists of replacing the contraction finder module of BASELINE by those proposed here and verifying the grammar checker effectiveness using annotated corpora. BASELINE is the CoGrOO 3.1.2 with the new sentence boundary detector, tokenizer and proper name finder, proposed in Sections 6.2, 6.3 and 6.4. 


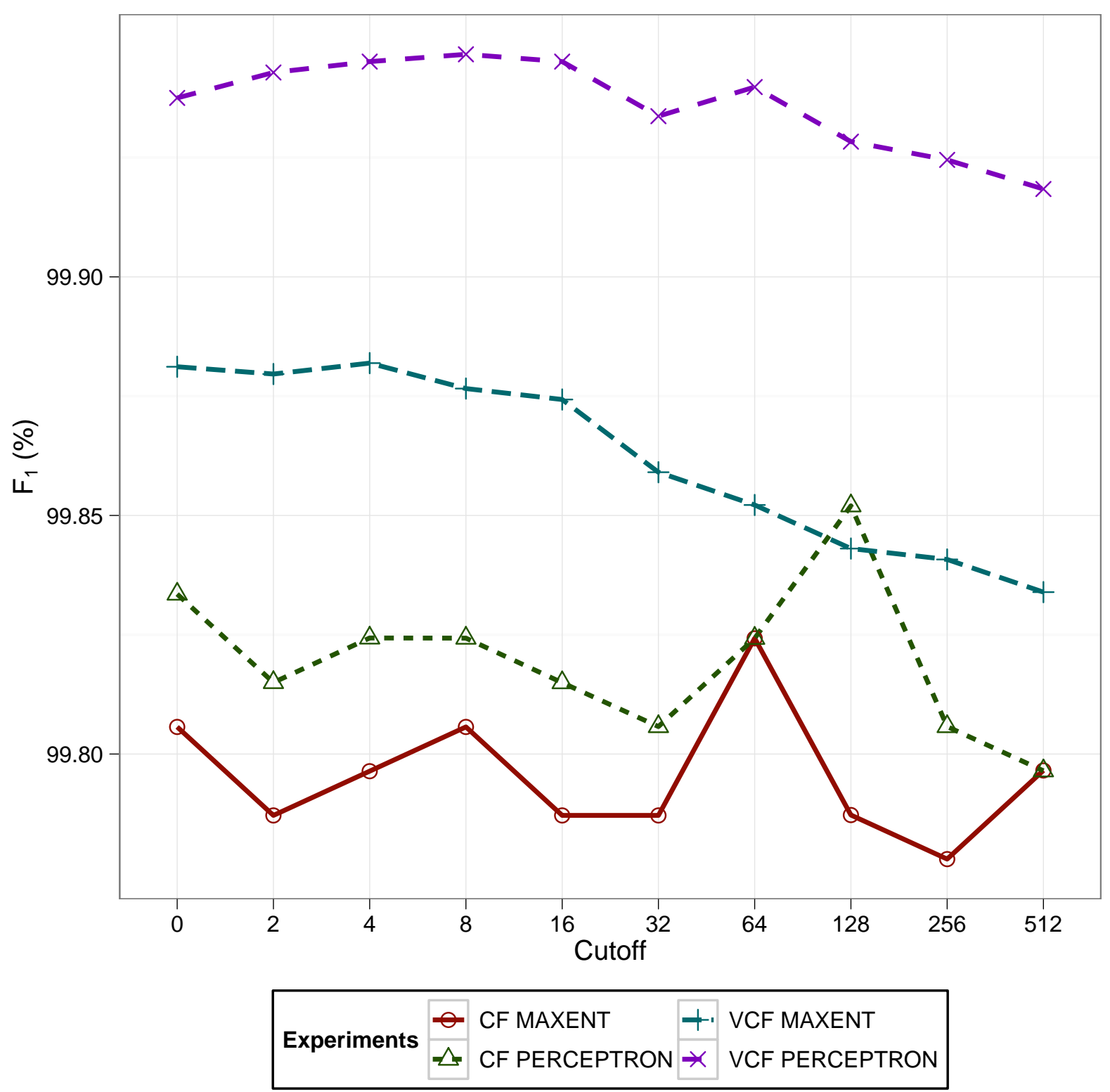

Figure 6.5: Distribution of the $F_{1}$ score according to the contraction finder configuration and the cutoff.

The results are summarized in Table 6.23. The following sections present the results.

\section{Metro Corpus}

No configurations of the new contraction name finder caused any change in the evaluation results for the Metro Corpus.

\section{Probi and Bosque Corpus}

All new contraction finder configurations increased the total number of false positives. This is bad at first sight, but actually it is positive from the perspective of the contraction finder itself, because now it can better distinguish the contraction nos from the pronoun. 


\begin{tabular}{|c|c|c|c|c|c|c|c|}
\hline & \multicolumn{3}{|c|}{ Metro } & \multicolumn{3}{|c|}{ Probi } & Bosque \\
\hline Experiment & Target & $\mathrm{TP}$ & $\mathrm{FP}$ & Target & $\mathrm{TP}$ & $\mathrm{FP}$ & FP \\
\hline BASELINE & & 16 & 33 & & 328 & 605 & 243 \\
\hline VCF PERCEPTRON 8 & & 16 & 33 & & $\begin{array}{r}329 \\
+1\end{array}$ & $\begin{array}{r}611 \\
+10 \\
-4\end{array}$ & $\begin{array}{r}243 \\
+2 \\
-2\end{array}$ \\
\hline VCF MAXENT 4 & 53 & 16 & 33 & 2616 & 328 & $\begin{array}{r}609 \\
+4\end{array}$ & $\begin{array}{r}245 \\
+2\end{array}$ \\
\hline CF PERCEPTRON 128 & & 16 & 33 & & $\begin{array}{r}329 \\
+1\end{array}$ & $\begin{array}{r}609 \\
+6 \\
-2\end{array}$ & $\begin{array}{r}244 \\
+2 \\
-1\end{array}$ \\
\hline CF MAXENT 64 & & 16 & 33 & & $\begin{array}{r}329 \\
+1\end{array}$ & $\begin{array}{r}606 \\
+4 \\
-3 \\
\end{array}$ & $\begin{array}{r}244 \\
+2 \\
-1 \\
\end{array}$ \\
\hline
\end{tabular}

Table 6.23: Summary of the grammar checker evaluation using different configuration of the contraction finder module. The number in the experiment name refers to the cutoff value used. Bosque corpus does not include grammar error annotations. Signed numbers refer to removed (when negative) or added (when positive) entries.

Every introduced false positives as well as the removed false positive and new true positive are related to nos.

Example 6.24 shows a common pattern of a removed false positive. BASELINE was expanding the pronoun nos as if it were a contraction, leading to the preposition em followed by the determiner os. This token rearrangement lead the POS tagger to classify leva as a singular, feminine noun, causing a false positive because the determiner did not agree with the name. The new contraction finder prevented nos from being expanded avoiding the error. Also, with the correct tokens, the POS tagger correctly classified nos as a pronoun and leva as a verb.

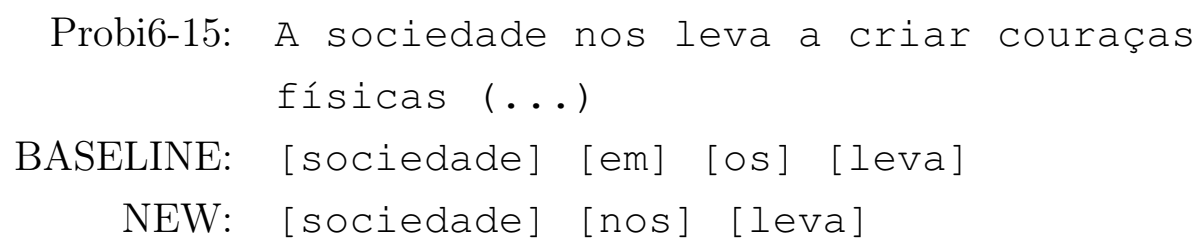

False positive: disagreement between plural, male determiner os and singular female noun leva

Also there is a common pattern of introduced false positive, when the improved contraction finder triggers a false positive that was latent because of another module. This is the case of Example 6.25. With the new contraction finder, the shallow parser wrongly classified nos as the subject of the verb criticou, causing a false positive. The old contraction finder was preventing this error because it was expanding nos. Many other introduced false 
positives are similar to this.

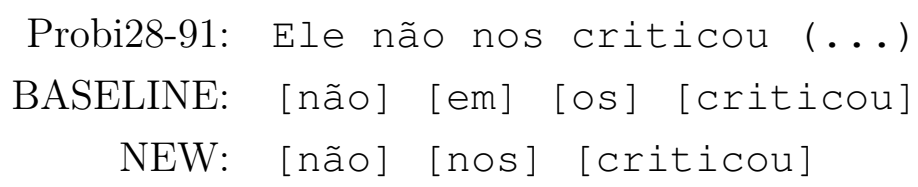

False positive: disagreement between plural subject nos and singular verb criticou

The new true positive, Example 6.26, is actually a new false positive. The real error in the example is the usage of the plural form of the verb haver in this context, houvessem should be replaced by houvesse. The grammar checker pointed out this misidentification of nos, here wrongly classified as the phrase subject by the shallow parser, and the verb teria. This is another example of the latent error described before.

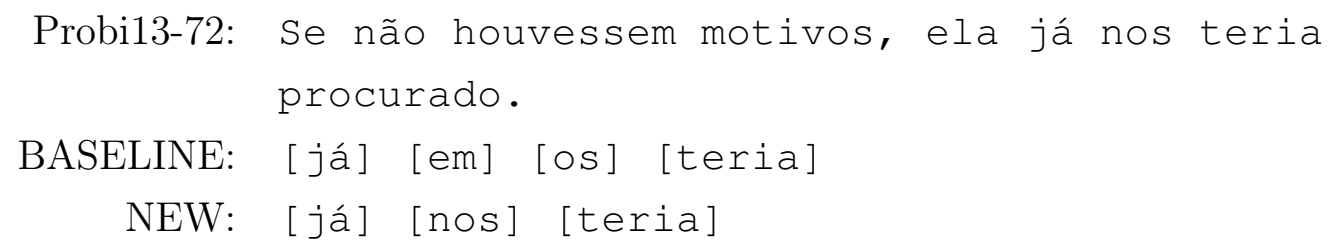

False positive: disagreement between plural subject nos and singular verb teria

All the other issues are similar to those already mentioned. Depending on the the configuration, the contraction finder could better differentiate contractions from pronouns, causing different results.

\subsubsection{Conclusion}

With the experimental results of the grammar checker using the new contraction finder, it is clear which module configuration is more effective for the final accuracy of the grammar checker. Analyzing the output of the module it is noticeable that it could differentiate a contraction, for example, the pronoun nos from the contraction em + os. Considering the 10-fold cross-validation, model size, and grammar checker effectiveness, the best model is:

- Algorithm: Perceptron

- Contraction dictionary: yes

- Corpus: Virgem CETENFolha

- Cutoff: eight 


\subsection{POS Tagger}

The part-of-speech (POS) tagger, as described in Section 3.1.4, selects a morphological class for each token in a sentence according to the token's context. For example, it will differentiate a verb from a noun, or an article from a preposition. In addition to the morphological class, the POS tagger implemented in CoGrOO 3.1.2 could assign morphological tags and features, like gender, number and tense, at once, but the high number of possible outcomes dramatically decreases the accuracy of the module.

The present work proposes doing this task in two steps. The first step uses the Apache OpenNLP POS Tagger to assign a morphological class to each token. The second step, called Featurizer and detailed in Section 6.7, takes as input the token sequence, as well as the assigned morphological classes, to choose the best features for each token.

The Apache OpenNLP POS tagger is based on one proposed by Ratnaparkhi (1998, Sec. 5) and works as follows:

- The module receives a sequence of tokens and optionally some additional context information;

- The token and its context are analyzed and the best morphological tag is assigned to each token.

The experiments were spliced in three, the first uses the default context generator proposed by Apache OpenNLP, the second includes additional context information coming from other modules, and the final features specially designed to tackle Portuguese particularities.

\subsubsection{Multi-Word Expressions}

Multi-word expressions, introduced in Section 3.1.4, are frequent in the training corpora. The following tagging schema was used to represent MWE: the tag of the first lexeme of the MWE receives a $B$ - representing the beginning of a $M W E$, and the subsequent tags receives a I- representing the continuation of a MWE. This is shown in Example 6.27.

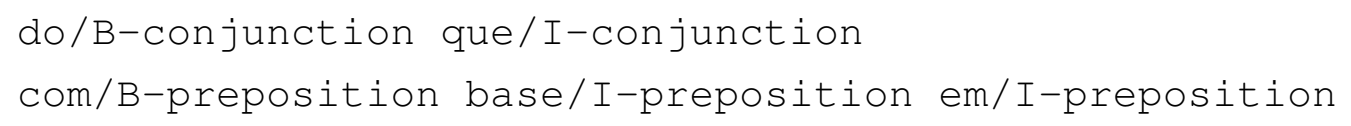

\subsubsection{Sequence Validation and the POS Dictionary}

A sequence validator is responsible for checking if a determined sequence of outcomes is valid. In the case of the POS Tagger, the sequence validator has to check whether the sequence of MWE are coherent, for example validating whether all boundary tags are followed by an intermediate tag, and if the an outcome is valid for a given lexeme. 
This last validation is achieved with the help of a POS dictionary, which is compiled from JSpell.br (Section 2.3.1). If a lexeme is present in the dictionary, only the morphological classes associated with its entry are accepted as a valid outcome. It increases the precision of the POS Tagger, but on the other hand the quality of the dictionary is crucial to the accuracy of the module.

\subsubsection{Default Context Generator}

The default POS Tagger implementation relies on a number of contextual templates. Lets define Lexeme the token under analysis. The contextual templates have the following form:

- The lexeme, its suffixes and prefixes;

- Whether the lexeme has capital letter, numbers or special characters;

- The surrounding context, which is composed of the two previous tokens and the two following ones;

- The outcomes selected for the last two tokens.

The contextual predicates are the combination of the contextual template and the actual data. For example, for the input A Maria chegou em a casa. (Maria arrived at home.), suppose that the token under analysis is chegou and the previously selected outcomes are article for A and proper noun for Maria. The contextual predicates would be:

- lexeme $=$ chegou, suffi $x_{1}=u$, suffi $x_{2}=$ ou, suffi $x_{3}=$ gou, suffi $x_{4}=$ egou, prefix $x_{1}=c$, prefix $x_{2}=c h$, prefix $_{3}=$ che, prefix $_{4}=$ cheg;

- previous $_{1}=$ Maria, previous $_{2}=A$ following $_{1}=$ em, following ${ }_{2}=a$

- outcome $_{1}=$ proper noun, outcome $e_{2}=$ article

\section{Experiments}

A set of 10-fold cross-validation experiments were carry out using the default contextual predicates. Each experiment combines:

- An algorithm (MAXENT or PERCEPTRON);

- Whether to use or not the POS dictionary derived from Jspell.br (DICT);

- Whether to create or not a mutable POS dictionary generated from the training data (MDICT). 


\begin{tabular}{|c|c|c|c|c|c|c|c|c|}
\hline 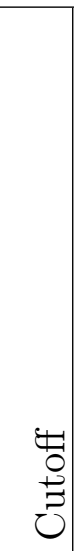 & 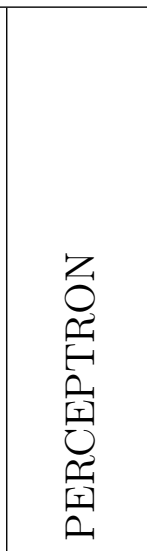 & 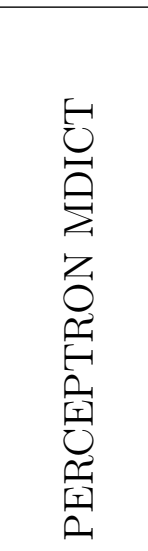 & 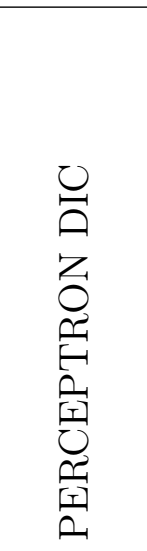 & 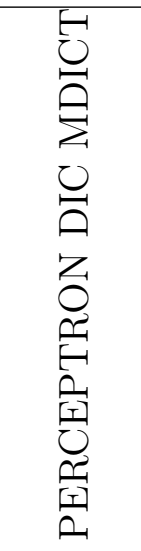 & 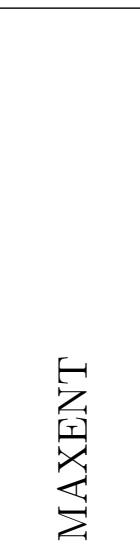 & 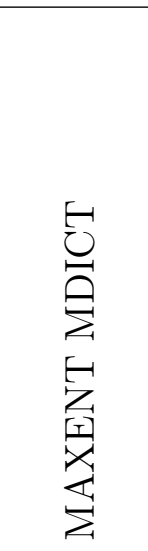 & 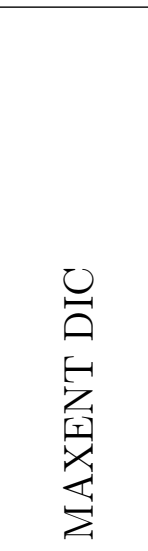 & 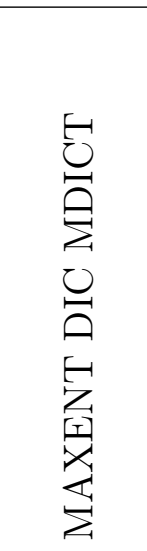 \\
\hline 0 & 94.672 & 95.156 & 93.411 & 95.473 & 94.868 & 95.176 & 94.359 & 95.848 \\
\hline 2 & 94.231 & 94.823 & 93.063 & 95.178 & 94.906 & 95.334 & 94.422 & 95.947 \\
\hline 4 & 93.681 & 94.424 & 92.750 & 94.749 & 94.842 & 95.463 & 94.461 & $\underline{95.979}$ \\
\hline 8 & 93.207 & 94.185 & 92.433 & 94.448 & 94.575 & 95.400 & 94.364 & 95.897 \\
\hline 16 & 92.080 & 93.304 & 91.695 & 93.642 & 94.077 & 95.243 & 94.098 & 95.685 \\
\hline 32 & 90.588 & 92.425 & 90.950 & 92.772 & 92.997 & 94.728 & 93.472 & 95.102 \\
\hline 64 & 89.208 & 92.153 & 90.674 & 92.475 & 91.723 & 94.364 & 93.038 & 94.711 \\
\hline 128 & 87.828 & 91.747 & 90.313 & 92.109 & 90.028 & 93.989 & 92.647 & 94.315 \\
\hline 256 & 84.379 & 89.683 & 88.002 & 90.012 & 87.371 & 93.275 & 91.869 & 93.605 \\
\hline 512 & 80.743 & 89.546 & 88.243 & 90.209 & 83.656 & 92.654 & 91.398 & 93.112 \\
\hline
\end{tabular}

Table 6.24: 10-fold cross validation results for different configurations of the POS tagger. The highest accuracy of each configuration is in bold, and the overall highest score is underlined.

Each of the 12 combinations were evaluated using different cutoff values: $0,2,4,8,16$, 32, 64, 128, 256 and 512. Table 6.24 summarizes the experiment results.

The highest accuracy was $95.979 \%$ obtained by a Maxent model trained with cutoff 4 , using the Jspell.br dictionary expanded with entries extracted from the training data. Figure 6.6 shows how the accuracy scores are distributed according to the configuration and the cutoff.

Maxent models performed slightly better compared to Perceptron models in this experiment and in both cases the presence of a dictionary drastically changed the effectiveness of the module.

With a mutable dictionary the module performs $0.5 \%$ better than without it, but using the Jspell.br dictionary caused a degradation in the results that surpasses $1 \%$ in a Perceptron model and $0.5 \%$ in a Maxent model. This behaviour can be explained by divergences between lexeme classifications in the training data and in the Jspell.br dictionary. The combination of the two dictionaries improved the results by $1 \%$.

\subsubsection{Additional Context}

Being part of a pipeline, the POS tagger is executed later than the Name Finder and the Contraction Finder modules. These modules can provide clues about previously taken 

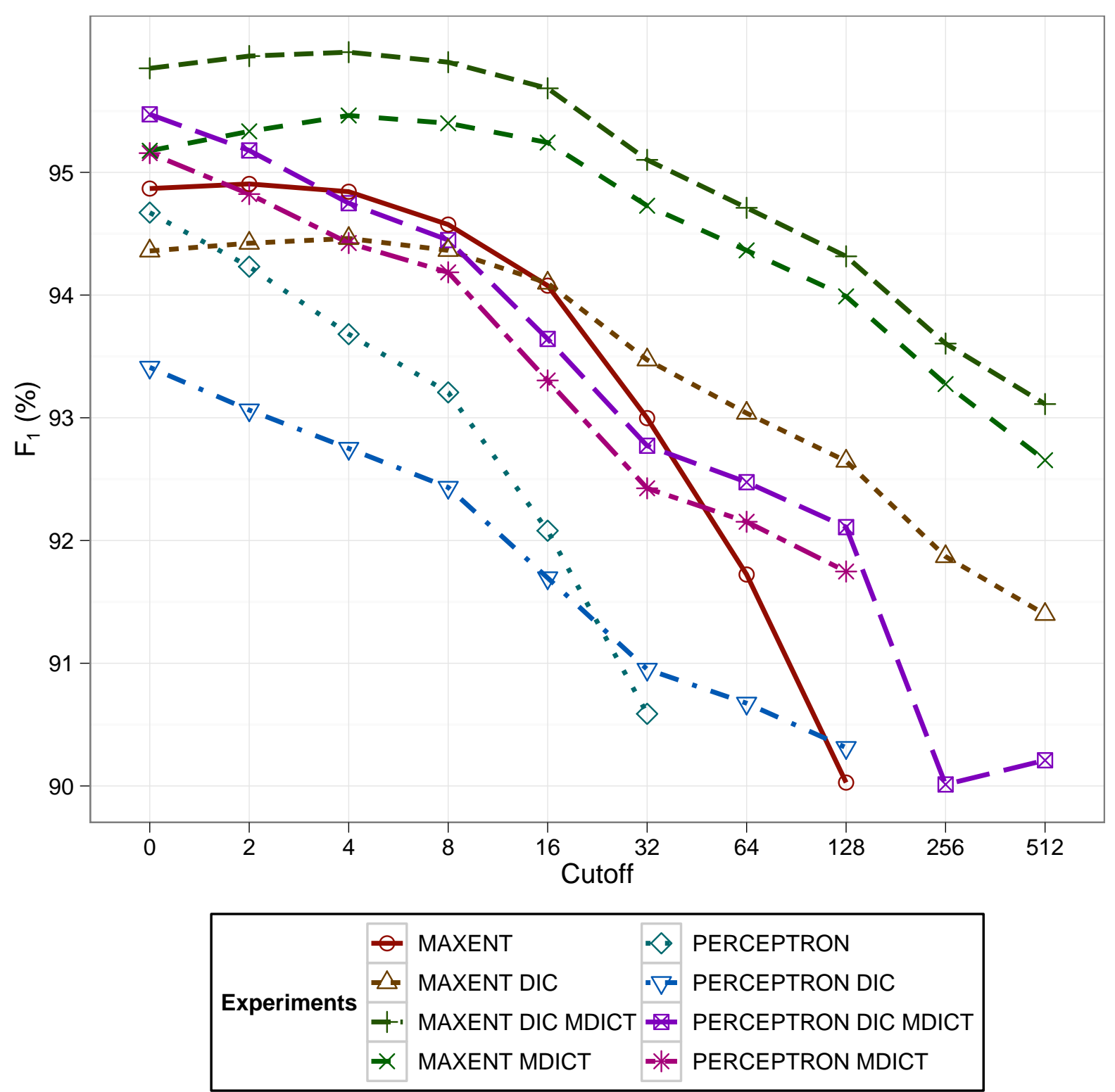

Figure 6.6: Distribution of the accuracy according to the POS Tagger configuration and the cutoff.

decisions; for example, if the token under analysis was classified as a proper name, or if it is an expanded contraction.

Apache OpenNLP allows passing this information to the POS Tagger as additional context and the Default Context Generator can be customized to include these data while computing the contextual predicates.

\section{Experiments}

To evaluate the impact of the additional context, a set of cross-validation experiments were carried out. Each experiment combines:

- An algorithm (MAXENT or PERCEPTRON); 
- Whether to use or not the additional context information (AC).

- Whether to use or not the fixed POS dictionary (Jspell.br) complemented with the mutable POS dictionary generated from the training data (DIC and MDICT).

\begin{tabular}{|c|c|c|c|c|c|c|c|c|}
\hline 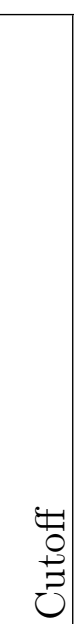 & 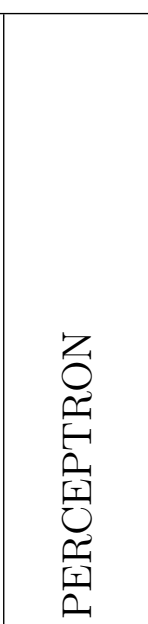 & 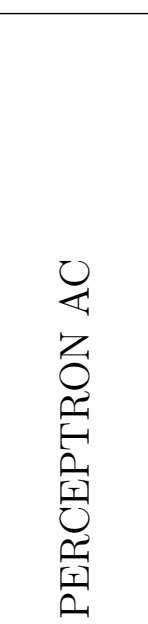 & 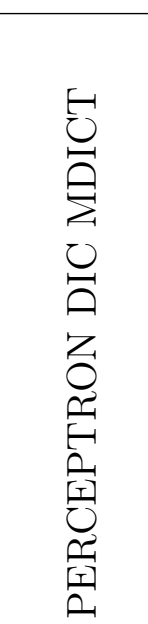 & 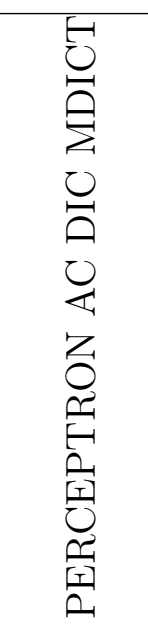 & 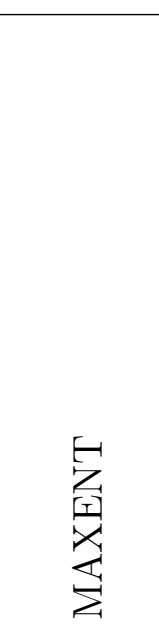 & 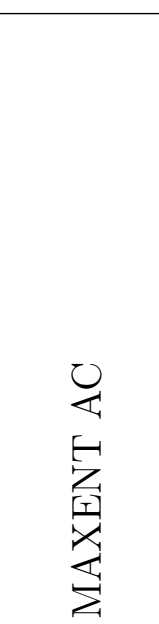 & 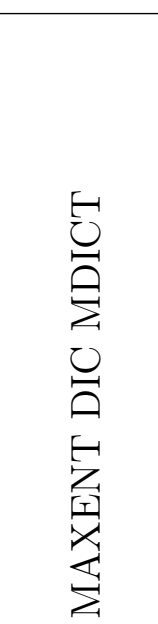 & 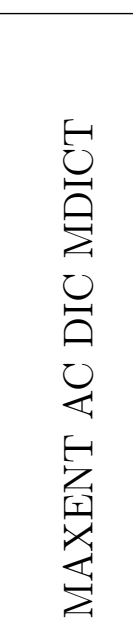 \\
\hline 0 & 94.672 & .046 & 95.473 & 95.844 & 94.868 & 94.951 & 95.848 & 95.923 \\
\hline 2 & 94.231 & 94.623 & 95.178 & 95.520 & 94.906 & 95.046 & 95.947 & 96.062 \\
\hline 4 & 93.681 & 94.150 & 94.749 & 95.172 & 94.842 & 94.988 & 95.979 & $\underline{96.091}$ \\
\hline 8 & 93.207 & 93.653 & 94.448 & 94.930 & & 94.755 & 95.897 & 96.044 \\
\hline 16 & 92.080 & 92.771 & 93.642 & 94.273 & 94.077 & 94.250 & 95.685 & 95.824 \\
\hline 32 & 90.588 & 91.165 & 92.772 & 93.283 & 92.997 & 93.249 & 95.102 & 95.285 \\
\hline 64 & 89.208 & 89.951 & 92.475 & 93.010 & 91.723 & 92.002 & 94.711 & 94.886 \\
\hline 128 & 87.828 & 88.200 & 92.109 & 92.497 & 90.028 & 90.286 & 94.315 & 94.488 \\
\hline 256 & 84.379 & 84.848 & 90.012 & 90.596 & 87.371 & 87.643 & 93.605 & 93.787 \\
\hline 512 & 80.743 & 81.160 & 90.209 & 90.866 & 83.656 & 83.987 & 93.112 & 93.255 \\
\hline
\end{tabular}

Table 6.25: 10-fold cross validation results for different configurations of the POS tagger. The highest accuracy of each configuration is in bold, and the overall highest score is underlined.

The highest accuracy was $96.091 \%$ obtained by a Maxent model trained with cutoff 4 and the additional context information in addition to the Jspell.br dictionary expanded with entries extracted from the training data. Figure 6.7 shows how the accuracy scores are distributed according to the configuration and the cutoff.

The additional context improved the results in a Maxent model by only $0.1 \%$. The Perceptron models performed better, by approximately $0.4 \%$.

Table 6.26 shows in detail the impact of the additional context. Only the most affected tags are included; they are prop (proper name), prp (preposition) and art (article). These tags are the most affected because the additional context information is derived precisely from modules that deal with proper names and contractions.

Although the change is apparently minor, only an evaluation of the effectiveness of the grammar checker will clearly indicate whether this improvement is relevant or not. 

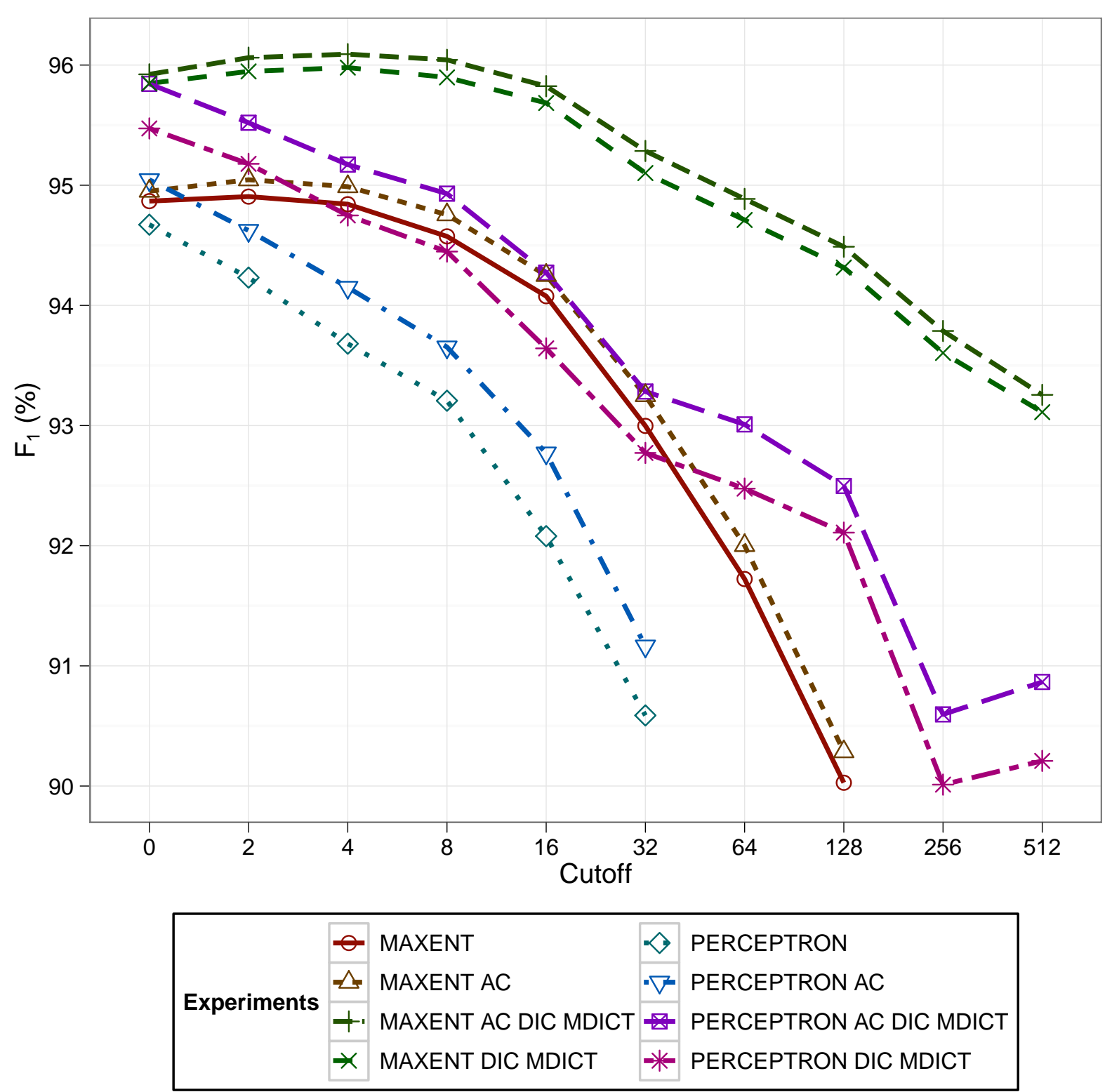

Figure 6.7: Distribution of the accuracy according to the POS Tagger configuration and the cutoff.

\begin{tabular}{|c|c|c|c|c|c|c|c|c|c|}
\cline { 3 - 9 } \multicolumn{2}{c|}{} & \multicolumn{2}{c|}{ Maxent } & \multicolumn{2}{c|}{ Maxent AC } & \multicolumn{2}{c|}{ Perceptron } & \multicolumn{2}{c|}{ Perceptron AC } \\
\hline Tag & Count & Errors & $\%$ Err & Errors & $\%$ Err & Errors & \% Err & Errors & $\%$ Err \\
\hline prop & 4723 & 280 & $5.93 \%$ & 235 & $4.98 \%$ & 184 & $3.90 \%$ & 170 & $3.60 \%$ \\
prp & 11835 & 182 & $3.85 \%$ & 164 & $3.47 \%$ & 339 & $7.18 \%$ & 231 & $4.89 \%$ \\
art & 11068 & 104 & $2.20 \%$ & 94 & $1.99 \%$ & 93 & $1.97 \%$ & 105 & $2.22 \%$ \\
\hline
\end{tabular}

Table 6.26: Error rate comparison between models trained with and without additional context. The classes prop, prp and art are the mostly affected because the additional context information comes from the Name Finder and Contraction Finder modules. 


\subsubsection{Custom Context Generator}

The lexemes with the highest number of errors are a and que. Such errors are related to high ambiguity of these lexemes and the lack of context to determine between the possible classes.

\begin{tabular}{|c|c|c|c|c|c|c|c|c|c|}
\cline { 3 - 10 } \multicolumn{2}{c|}{} & \multicolumn{2}{c|}{ Maxent } & \multicolumn{2}{c|}{ Maxent CCG } & \multicolumn{2}{c|}{ Perceptron } & \multicolumn{2}{c|}{ Perceptron CCG } \\
\hline Token & Count & Errors & $\%$ Err & Errors & $\%$ Err & Errors & $\%$ Err & Errors & $\%$ Err \\
\hline a & 4503 & 253 & $5.62 \%$ & 203 & $4.51 \%$ & 215 & $4.77 \%$ & 145 & $3.22 \%$ \\
que & 1435 & 194 & $13.52 \%$ & 194 & $13.52 \%$ & 272 & $18.95 \%$ & 214 & $14.91 \%$ \\
\hline
\end{tabular}

Table 6.27: Error rate comparison for the lexemes a and que between models trained with and without the customized context generator.

To help the POS Tagger determine whether the lexeme a is a preposition or an article the following contextual templates were designed:

- The transitivity of the previous, current and next tokens (verbs only);

- Whether the previous token were classified as a verb;

- If the previous token is a verb, add its lemma;

- Whether if the next token can be classified as a feminine noun.

These contextual templates are only evaluated if the current lexeme is a. To accomplish this, a dictionary containing transitivity and gender information was derived from Jspell.br.

Another set of contextual templates were designed to help the tagger determine if the lexeme que is a pronoun or a conjunction:

- If the two previous tokens were classified as a verb;

- If the next token can be classified as a verb.

\section{Experiments}

Another set of cross-validation experiments were carried out to evaluate if the custom context generator helps the POS tagger disambiguate the lexemes a and que. Table 6.28 shows the evaluation results.

The highest accuracy was $96.291 \%$ obtained by a Perceptron model trained with the customized context generator with cutoff 0, additional context information, the Jspell.br dictionary expanded with entries extracted from the training data. Figure 6.8 shows how the accuracy scores are distributed according to the configuration and the cutoff.

The additional features for a and que improved the results in a Perceptron model by something around $0.6 \%$ in a simple model and $0.4 \%$ in a model that also includes dictionaries 


\begin{tabular}{|c|c|c|c|c|c|c|c|c|}
\hline 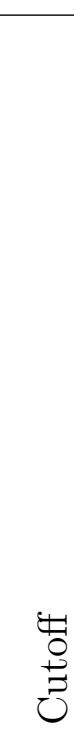 & 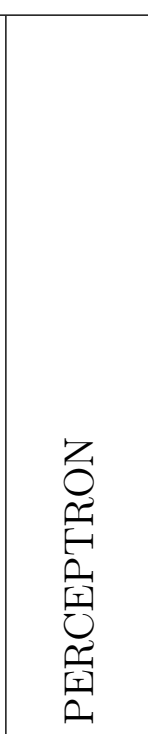 & 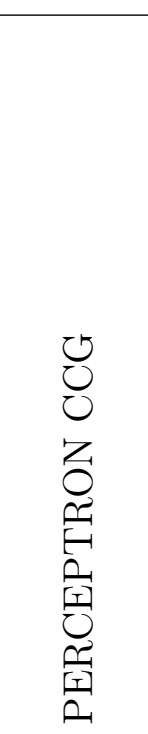 & 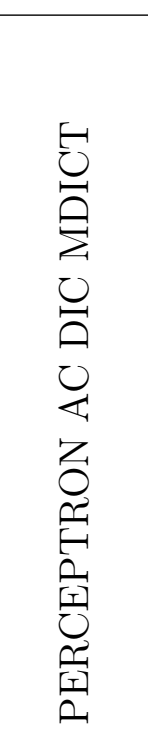 & 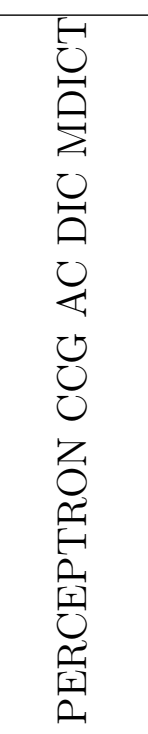 & 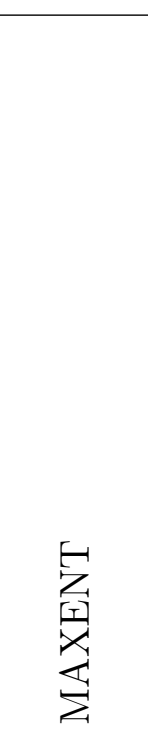 & 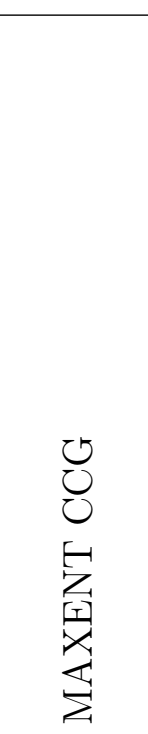 & 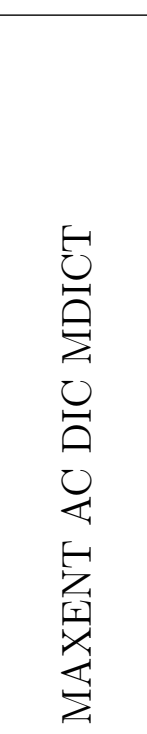 & 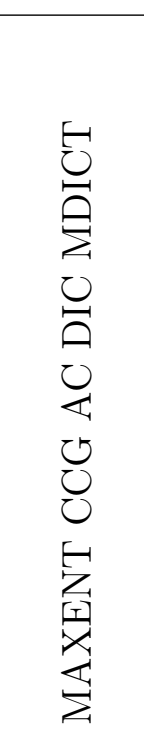 \\
\hline 0 & 94.672 & 95.335 & 95.844 & 96.291 & 94.868 & 95.013 & 95.923 & 96.019 \\
\hline 2 & 94.231 & 95.048 & 95.520 & 96.045 & 94.906 & 95.088 & 96.062 & 96.137 \\
\hline 4 & 93.681 & 94.659 & 95.172 & 95.760 & 94.842 & 95.019 & 96.091 & 96.129 \\
\hline 8 & 93.207 & 94.163 & 94.930 & 95.454 & 94.575 & 94.816 & 96.044 & 96.115 \\
\hline 16 & 92.080 & 93.084 & 94.273 & 94.892 & 94.077 & 94.295 & 95.824 & 95.891 \\
\hline 32 & 90.588 & 91.527 & 93.283 & 93.961 & 92.997 & 93.379 & 95.285 & 95.433 \\
\hline 64 & 89.208 & 89.725 & 93.010 & 93.333 & 91.723 & 92.229 & 94.886 & 95.027 \\
\hline 128 & 87.828 & 88.448 & 92.497 & 92.750 & 90.028 & 90.675 & 94.488 & 94.659 \\
\hline 256 & 84.379 & 86.080 & 90.596 & 91.150 & 87.371 & 88.490 & 93.787 & 93.885 \\
\hline 512 & 80.743 & 82.287 & 90.866 & 90.778 & 83.656 & 85.424 & 93.255 & 93.264 \\
\hline
\end{tabular}

Table 6.28: 10-fold cross validation results for different configurations of the POS tagger. The highest accuracy of each configuration is in bold, and the overall highest score is underlined.

and additional context information. The Maxent models had a smaller improvement, by something around $0.2 \%$ in a simple model, and less than $0.05 \%$ in a model that also includes dictionaries and additional context information.

Table 6.26 shows in detail the impact of the additional context. Only the most affected tags are included; they are prop (proper name), prp (preposition) and art (article). These tags are the most affected because the additional context information is derived precisely from modules that deal with proper names and contractions.

Although the change is apparently minor, only an evaluation of the effectiveness of the grammar checker will tell for sure if this improvement is relevant or not.

\subsubsection{Multi-Word Expressions Results}

Table 6.29 details the effectiveness of the POS tagger classifying multi-word expressions. The numbers are for the model PERCEPTRON DIC MDICT CCG AC 0 .

The zero scores for some classes are related to the low number of available training data. 

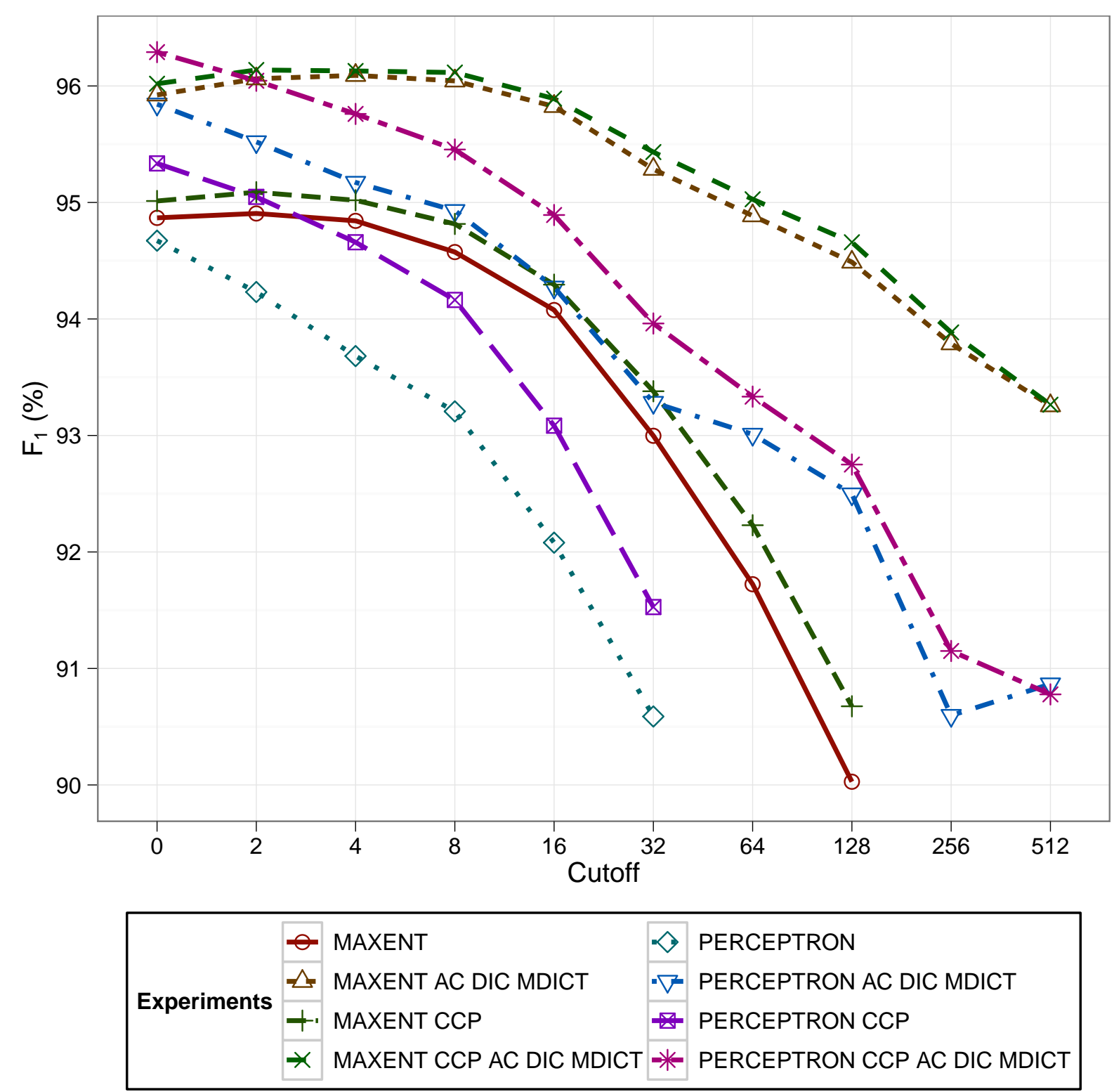

Figure 6.8: Distribution of the accuracy according to the POS Tagger configuration and the cutoff.

\subsubsection{Previous Work}

To compare the results obtained by this work with others is not a simple task because the corpus used for training and evaluation plays an important role in the scores. In this work the original corpus was used as is, not manipulated, fixed or improved.

In Portuguese we can compare the results with the VLMM tagger (Section 2.2.2), which scored $96.673 \%$, and the one proposed by Silva (Section 2.2.4), that scored $96.87 \%$. The tagger proposed here obtained a lower accuracy compared to the other two approaches, $96.291 \%$, but as mentioned before, comparing the accuracy is not sufficient to determine which is better. An evaluation following the same criteria and against the same corpus would be better, but this will be left for future work to determine. 


\begin{tabular}{|c|c|c|c|c|c|c|}
\hline MWE & Precision & Recall & $F_{1}$ & Target & TP & FP \\
\hline prp & $85.33 \%$ & $79.50 \%$ & $82.31 \%$ & 278 & 221 & 38 \\
conj-s & $72.22 \%$ & $68.42 \%$ & $70.27 \%$ & 57 & 39 & 15 \\
num & $66.67 \%$ & $66.67 \%$ & $66.67 \%$ & 6 & 4 & 2 \\
pron-indp & $55.65 \%$ & $83.12 \%$ & $66.67 \%$ & 77 & 64 & 51 \\
adv & $67.13 \%$ & $63.40 \%$ & $65.21 \%$ & 377 & 239 & 117 \\
n & $21.25 \%$ & $29.31 \%$ & $24.64 \%$ & 58 & 17 & 63 \\
adj & $0.00 \%$ & $0.00 \%$ & $0.00 \%$ & 10 & 0 & 2 \\
intj & $0.00 \%$ & $0.00 \%$ & $0.00 \%$ & 4 & 0 & 8 \\
pp & $0.00 \%$ & $0.00 \%$ & $0.00 \%$ & 1 & 0 & 0 \\
pron-det & $0.00 \%$ & $0.00 \%$ & $0.00 \%$ & 2 & 0 & 0 \\
\hline
\end{tabular}

Table 6.29: Effectives of the POS tagger classifying MWE. Evaluated 4212 samples with 870 expressions. Found 880, of which 584 were correct.

\subsubsection{Evaluating the Grammar Checker}

The evaluation consists of replacing the POS Tagger module of BASELINE by those proposed here and verifying the grammar checker effectiveness using annotated corpora. $B A S E L I N E$ is the CoGrOO 3.1.2 with the new sentence boundary detector, tokenizer, proper name finder and contraction finder, proposed in the previous sections. Table 6.30 summarizes the results.

Because it is difficult to replace the POS Tagger of CoGrOO 3.1.2 with the new one keeping the Chunker and Shallow Parser modules due to the tag set changes, these two modules were disabled in both BASELINE and the pipelines under evaluation. This implies that phrase-local and subject-verb rules will not be evaluated in this experiment.

The POS Tagger from BASELINE was also responsible for assigning feature tags to the tokens, but now this task was spliced and performed by a dedicated module. To make the evaluation possible, a Featurizer module was introduced into the pipelines under evaluation. The chosen featurizer was trained with the Perceptron algorithm, the standard feature generator and a dictionary in the sequence validator (PERCEPTRON DIC S). The Featurizer module will be described in detail in the next section.

\section{Metro Corpus}

Table 6.31 shows the evaluation results in terms of precision, recall and $F_{1}$. The recall values did not change with exception to perceptron models with the custom context generator $(\mathrm{CCG})$, but the precision varies significantly according to the configuration. The only model that surpassed BASELINE was MAXENT DIC MDIC CCG AC 2.

True positives In only two configurations the number of true positives changed, both configurations are perceptron models that include the Jspell.br dictionary (DIC) and the mutable dictionary (MDIC), as well as additional context information (AC). The only dif- 


\begin{tabular}{|c|c|c|c|c|c|c|c|}
\hline & \multicolumn{3}{|c|}{ Metro } & \multicolumn{3}{|c|}{ Probi } & Bosque \\
\hline Experiment & Target & $\mathrm{TP}$ & $\mathrm{FP}$ & Target & $\mathrm{TP}$ & $\mathrm{FP}$ & $\mathrm{FP}$ \\
\hline BASELINE & & 16 & $\overline{6} 6$ & & 233 & 204 & 100 \\
\hline $\begin{array}{l}\text { PERCEPTRON DIC MDIC CCG AC } \\
0\end{array}$ & & -1 & $\begin{array}{r}6 \\
+3 \\
-3\end{array}$ & & $\begin{array}{r}260 \\
+50 \\
-23\end{array}$ & $\begin{array}{r}139 \\
+48 \\
-112\end{array}$ & $\begin{array}{r}49 \\
+10 \\
-61\end{array}$ \\
\hline MAXENT DIC MDIC CCG AC 2 & & 16 & $\begin{array}{r}5 \\
+1 \\
-2\end{array}$ & & $\begin{array}{r}269 \\
+52 \\
-16\end{array}$ & $\begin{array}{r}162 \\
+53 \\
-94\end{array}$ & $\begin{array}{r}55 \\
+12 \\
-57\end{array}$ \\
\hline MAXENT DIC MDIC AC 4 & & 16 & $\begin{array}{r}8 \\
+4 \\
-2\end{array}$ & & $\begin{array}{r}267 \\
+53 \\
-19\end{array}$ & $\begin{array}{r}221 \\
+102 \\
-84\end{array}$ & $\begin{array}{r}58 \\
+14 \\
-56\end{array}$ \\
\hline PERCEPTRON DIC MDIC AC 0 & & $\begin{array}{l}15 \\
-1\end{array}$ & $\begin{array}{r}10 \\
+6 \\
-2\end{array}$ & & $\begin{array}{r}260 \\
+51 \\
-24\end{array}$ & $\begin{array}{r}226 \\
+111 \\
-88\end{array}$ & $\begin{array}{r}50 \\
+12 \\
-62\end{array}$ \\
\hline MAXENT DIC MDIC 4 & 53 & 16 & $\begin{array}{r}7 \\
+4 \\
-3\end{array}$ & 2616 & $\begin{array}{r}266 \\
+52 \\
-19\end{array}$ & $\begin{array}{r}214 \\
+96 \\
-85\end{array}$ & $\begin{array}{r}58 \\
+14 \\
-56\end{array}$ \\
\hline PERCEPTRON DIC MDIC 0 & & 16 & $\begin{array}{r}10 \\
+6 \\
-2\end{array}$ & & $\begin{array}{r}257 \\
+51 \\
-27\end{array}$ & $\begin{array}{r}252 \\
+138 \\
-89\end{array}$ & $\begin{array}{r}58 \\
+16 \\
-58\end{array}$ \\
\hline MAXENT MDIC 4 & & 16 & $\begin{array}{r}8 \\
+5 \\
-3\end{array}$ & & $\begin{array}{r}262 \\
+55 \\
-26\end{array}$ & $\begin{array}{r}237 \\
+128 \\
-94\end{array}$ & $\begin{array}{r}60 \\
+15 \\
-55\end{array}$ \\
\hline PERCEPTRON MDIC 0 & & 16 & $\begin{array}{r}11 \\
+7 \\
-2\end{array}$ & & $\begin{array}{r}252 \\
+53 \\
-34\end{array}$ & $\begin{array}{r}289 \\
+178 \\
-92\end{array}$ & $\begin{array}{r}59 \\
+17 \\
-58\end{array}$ \\
\hline MAXENT 2 & & 16 & $\begin{array}{r}9 \\
+6 \\
-3\end{array}$ & & $\begin{array}{r}266 \\
+60 \\
-27\end{array}$ & $\begin{array}{r}293 \\
+181 \\
-91\end{array}$ & $\begin{array}{r}61 \\
+17 \\
-56\end{array}$ \\
\hline PERCEPTRON 0 & & 16 & $\begin{array}{r}12 \\
+8 \\
-2\end{array}$ & & $\begin{array}{r}248 \\
+54 \\
-39\end{array}$ & $\begin{array}{r}307 \\
+191 \\
-87\end{array}$ & $\begin{array}{r}60 \\
+20 \\
-60 \\
\end{array}$ \\
\hline
\end{tabular}

Table 6.30: Summary of the grammar checker evaluation using different configuration of the partof-speech tagger. The number in the experiment name refers to the cutoff value used. Bosque corpus does not include grammar error annotations.

ference is that one includes the customized context generator (CCG), whereas the other does not. The change is a new false negative in sentence 6.28. The new pos tagger, which now is aware of multi-word expressions, classified the tokens ou seja (in other words) as a MWE and linked them together. The rule system was expecting it as two tokens and it caused the rule matcher to fail. The solution would be to modify the rule system to be aware of MWE 


\begin{tabular}{|l|r|r|r|}
\hline \multicolumn{1}{|c|}{ Experiment } & Precision & Recall & \multicolumn{1}{c|}{$F_{1}$} \\
\hline \hline BASELINE & 72.73 & $\mathbf{3 0 . 1 9}$ & 42.67 \\
\hline PERCEPTRON DIC MDIC CCG AC 0 & 71.43 & 28.30 & 40.54 \\
\hline MAXENT DIC MDIC CCG AC 2 & $\mathbf{7 6 . 1 9}$ & $\mathbf{3 0 . 1 9}$ & $\mathbf{4 3 . 2 4}$ \\
\hline MAXENT DIC MDIC AC 4 & 66.67 & $\mathbf{3 0 . 1 9}$ & 41.56 \\
\hline PERCEPTRON DIC MDIC AC 0 & 60.00 & 28.30 & 38.46 \\
\hline MAXENT DIC MDIC 4 & 69.57 & $\mathbf{3 0 . 1 9}$ & 42.11 \\
\hline PERCEPTRON DIC MDIC 0 & 61.54 & $\mathbf{3 0 . 1 9}$ & 40.51 \\
\hline MAXENT MDIC 4 & 66.67 & $\mathbf{3 0 . 1 9}$ & 41.56 \\
\hline PERCEPTRON MDIC 0 & 59.26 & $\mathbf{3 0 . 1 9}$ & 40.00 \\
\hline MAXENT 2 & 64.00 & $\mathbf{3 0 . 1 9}$ & 41.03 \\
\hline PERCEPTRON 0 & 57.14 & $\mathbf{3 0 . 1 9}$ & 39.51 \\
\hline
\end{tabular}

Table 6.31: Summary of the grammar checker evaluation against Metro corpus. The maximun value for precision and recall in bold.

as well and properly handle cases like this.

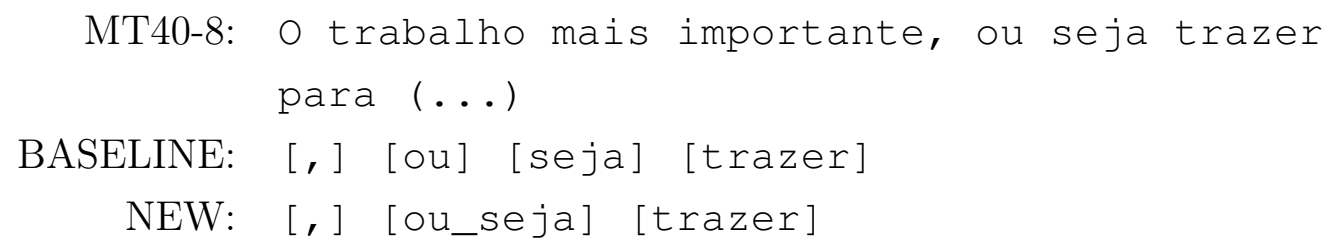

False negative: the expression ou seja should be surrounded by commas.

False positives The custom context generator (CCG) influenced the number of false positives. The Perceptron model removed three false positives compared to BASELINE, but added another three, whereas the Maxent model removed two false positives and added only one. Both models removed false positives from the sentences MT2-14 (Example 6.29) and MT20-5 (Example 6.30), but Perceptron also removed a false positive in the sentence MT153 (Example 6.31). The first was a false positive causing the a misclassification of the token Reconhecidas (Recognized), which in BASELINE was classified as a determiner, causing a discrepancy between a determiner and the following noun, but in fact it is an adjective, as pointed out by the new models. The second is related to the proper noun Vergueiro, which was classified by BASELINE as a noun, and now is correctly classified. This was causing a disagreement between the article and the noun. Finally, the false positive remove last is related the word cerca, which in the context should be an adverb, but was classified 
as a noun. Only the Perceptron model got this right.

\author{
MT2-14: Reconhecidas instituições de pesquisa (...) \\ BASELINE: det $n$ prp $n$ \\ NEW: adj $n$ prp $n$ \\ Removed FP: Reconhecidas was classified as an article, which should \\ agree with the following noun. Now it is correctly classified \\ as an adjective.
}

Removed FP: Vergueiro was classified as an noun, but should be a proper noun.

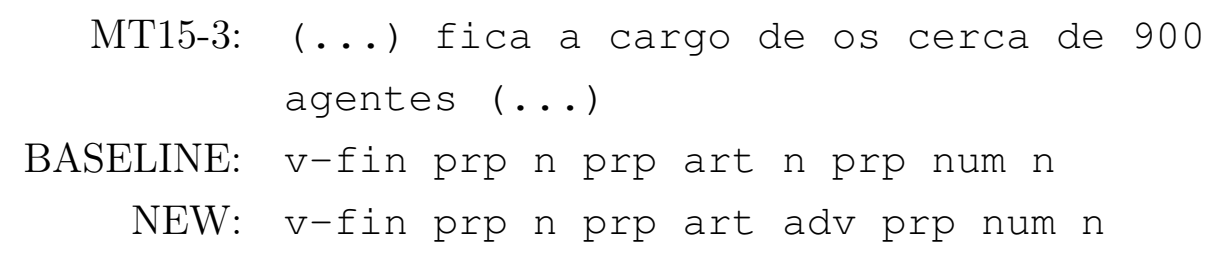

Removed FP: cerca was classified as an noun, but should be a adverb.

The models with CCG also added some new false positives. Both Perceptron and Maxent failed to classify the token presente in a presente política (the current policy) (MT12-4). In this context the token should be classified as an adjective, but was classified as a masculine noun. This caused a discrepancy with the preceding article. The Perceptron model caused two additional false positives. One was caused by the classification of the token a as an article instead of a preposition in the prepositional phrase a pé (on foot) (MT23-17). The other false positive was related to ordinal numbers: foi a 11 a Semana (was the 11th Week). The Perceptron model treated it as number, causing a conflict with the preceding article.

The number of new false positives grew worse by removing the CCG. New false positives, all related to the ambiguity of the lexeme a, which often causes a disagreement error if it was wrongly classified as an article instead of preposition. This is the case of the false positive in MT29-8, (...) a exemplo do que acontecia na Europa (...) (similar to what happened in Europe).

Removing the additional context (AC) had a similar effect in Maxent models. Without it, the POS Tagger misclassified another preposition in sentence MT23-10, ( . . ) restrita a pessoas (...) (restricted to people). The perceptron model without AC obtained it 
right.

Removing the Jspell dictionary (DIC) introduced another false positive. The tagger misclassified the lexeme abordou in (...) uma funcionária que os abordou (...) (an employee who approached them), sentence MT40-17. Without the dictionary the tagger classified it as a noun, but the only valid option for this token is a verb. Also, the tagger misclassified the preceding os, which was classified as an article instead of pronoun, and this caused a false positive. Removing the mutable dictionary (MDIC), other lexemes were misclassified, causing more false positives.

\section{Probi Corpus}

\begin{tabular}{|l|r|r|r|}
\hline \multicolumn{1}{|c|}{ Experiment } & Precision & Recall & \multicolumn{1}{c|}{$F_{1}$} \\
\hline \hline BASELINE & 53.32 & 8.91 & 15.26 \\
\hline PERCEPTRON DIC MDIC CCG AC 0 & $\mathbf{6 5 . 1 6}$ & 9.94 & 17.25 \\
\hline MAXENT DIC MDIC CCG AC 2 & 62.41 & $\mathbf{1 0 . 2 8}$ & $\mathbf{1 7 . 6 6}$ \\
\hline MAXENT DIC MDIC AC 4 & 54.71 & 10.21 & 17.20 \\
\hline PERCEPTRON DIC MDIC AC 0 & 53.50 & 9.94 & 16.76 \\
\hline MAXENT DIC MDIC 4 & 55.42 & 10.17 & 17.18 \\
\hline PERCEPTRON DIC MDIC 0 & 50.49 & 9.82 & 16.45 \\
\hline MAXENT MDIC 4 & 52.51 & 10.02 & 16.82 \\
\hline PERCEPTRON MDIC 0 & 46.58 & 9.63 & 15.96 \\
\hline MAXENT 2 & 47.58 & 10.17 & 16.76 \\
\hline PERCEPTRON 0 & 44.68 & 9.48 & 15.64 \\
\hline
\end{tabular}

Table 6.32: Summary of the grammar checker evaluation against Probi corpus. The maximun value for precision, recall and in bold.

Table 6.32 shows the evaluation results in terms of precision, recall and $F_{1}$. In terms of $F_{1}$, the best model was MAXENT DIC MDIC CCG AC 2, which scored $17.66 \%, 2.6 \%$ better than BASELINE.

The recall results obtained by the proposed tagger were always better than those obtained by BASELINE, while the precision grew worse removing the dictionaries. The recall values always increase with the complexity of the model, and Maxent models always have a higher recall score according to this experiment.

The precision of Maxent models are higher in this experiment with exception to when the Perceptron model with the custom context generator, which scored $2.75 \%$ more than the best Maxent model. Again, the scores always increase according to the complexity of the model.

Most of the issues of this corpus were already illustrated in the analysis of the evaluation using Metro corpus. 


\section{Bosque Corpus}

The evaluation of the new POS tagger with the Bosque corpus improved in the number of false positives, which decreased from 100 in the baseline to 49 in the best Perceptron model, and 55 in the best Maxent model. The value changed slightly between each configuration, but the variations follows the analysis already done for Probi and Metro corpora.

\subsubsection{The Ambiguity of the Preposition A Versus the Article A}

The experiments with the corpus highlighted the issue described in Section 6.6.5, the pos ambiguity of the lexeme a. To demonstrate its impact in the grammar checker, rule 114, which checks whether an article agrees with the following noun, was modified to disregard if the article is an a.

Table 6.33 summarizes the results. The ambiguity of a caused a high number of false positives, leading the precision to fall under $50 \%$ both in BASELINE and the new tagger. Changing the rule to ignore a classified as an article, the number of true positives slightly decreased, but the number of false positives decreased pronouncedly, so the precision greatly increased.

The changes in the new tagger improved the classification of a, but it is still not enough. Finding a better solution for this issue will be left for future studies.

\begin{tabular}{|l|c|c|c|c|c|c|c|}
\cline { 2 - 8 } \multicolumn{1}{c|}{} & \multicolumn{3}{c|}{ Metro } & \multicolumn{3}{c|}{ Probi } & Bosque \\
\cline { 2 - 8 } \multicolumn{1}{c|}{} & TP & FP & Precision & TP & FP & Precision & FP \\
\hline BASELINE & 6 & 4 & 0.6 & 43 & 107 & 0.29 & 76 \\
\hline NEW & 6 & 4 & 0.6 & 61 & 65 & 0.48 & 32 \\
\hline BASELINE -a & 3 & 1 & 0.75 & 40 & 55 & 0.42 & 47 \\
\hline NEW -a & 3 & 0 & 1 & 48 & 23 & 0.68 & 27 \\
\hline
\end{tabular}

Table 6.33: Evaluation results for the rule 104, which checks the agreement between an article and the following noun. The -a in the last two rows indicates that the rule were modified to be ignored if the article is an a.

\subsubsection{Conclusion}

In the 10-fold cross-validation, the Perceptron model obtained a better $F_{1}$ score than Maxent: 96.291 against 96.137. Customizing the context generator for Portuguese proved very effective, also a good quality dictionary contributed substantially.

To choose the best configuration considering the results from the grammar checker evaluation is quite challenging. The precision of Perceptron models are higher than Maxent models because of the lower number of false positives, while the recall goes in the opposite direction, because Maxent models provide more true positives. The $F_{1}$ combines precision and recall in only one score, and it would be fair to use the model with the best $F_{1}$. But it 
is important for a grammar checker to have the lowest number of false positives as possible, and Perceptron models performed much better in this regard than Maxent models.

Considering this, two aspects the following configuration was selected:

- Algorithm: Perceptron

- Mutable dictionary: yes

- Jspell dicionary: yes

- Additional context: yes

- Custom context generator: yes

- Cutoff: zero

The new POS Tagger will be incorporated to BASELINE and used during the evaluation of the other modules. 


\subsection{Featurizer}

As described in the previous section (Section 6.6), the task of handling inflection tagging is performed in a separate step by a dedicated module called a Featurizer. This approach is better than the extended POS tagset from CoGrOO 3, in which the tagger handles both morphological and inflection, because such an extended tagset is likely to lead to a lower tagging precision due to data-sparseness (Silva, 2007).

The Featurizer is not part of the Apache OpenNLP, but was created using the same framework so the usage is similar to other OpenNLP components.

The Featurizer works as follows:

- The module receives a sequence of tokens annotated with morphological tags;

- The context is analyzed and the best feature is assigned to the token.

\subsubsection{Sequence Validation and the Feature Dictionary}

A sequence validator, which is responsible for checking if a determined sequence of outcomes is valid, were added to the module to check if an outcome is valid for a given lexeme, as follows:

- Only valid combinations of morphological tag and feature are accepted, for example, a noun cannot have a tense;

- Validates if an outcome is possible for the token using a feature dictionary.

As example of the POS Tagger, a dictionary increases the precision of the module, but on the other hand the quality of the dictionary is crucial to its accuracy.

\subsubsection{Contextual Predicates}

The development of the Featurizer module requires the selection of the contextual templates. The contextual predicates are the combination of the contextual template and the actual data. The proposed contextual templates are:

Default The default contextual templates are extracted from a window of size three, including the lexeme under evaluation, the previous and the next lexemes. The templates have the following form:

- The previous, current and next lexeme;

- The previous, current and next POS tag;

- The outcomes selected for the previous token;

- The suffixes of the previous, current and next lexeme. 
Token Class (C) This additional contextual template consists of associating a class with each lexeme of the window. The classes are:

- lowercase alphabetic;

- two digits;

- four digits;

- alpha-numeric;

- digits and dashes;

- digits and slashes;

- digits and commas;

- digits and periods;

- digits;

- single capital letter;

- all capital letters;

- initial capital letter;

- other.

Number (N) This additional contextual template is specially designed to handle pure numbers. For each number of the window it will apply a template of the form:

- Whether the number is greater or equal to 2 ;

- Whether the number is between 1, inclusive and 2, exclusive;

- Whether the number is between 0 , inclusive and 1 , exclusive.

Hyphenated and Groups (H) This additional contextual template is specially designed to handle hyphenated words and group of words, like proper nouns that were joined by the Name Finder module. Each grouped or hyphenated lexeme of the context is separated and for each of its parts a basic suffix analysis is performed.

Wider window (W) The default contextual templates are extracted from a window of size 5 instead of 3 . 


\section{Experiments}

A set of 10-fold cross-validation experiments were carried out using the proposed contextual predicates. Each experiment combines:

- An algorithm (MAXENT or PERCEPTRON);

- Whether to use or not a fixed Feature Dictionary (Jspell.br);

- Combinations of the proposed contextual predicates.

The contextual predicates are represented by its alphabetic flag. The default contextual template is always applied. The flags are $\mathbf{C}$ for token class, $\mathbf{N}$ for number, $\mathbf{H}$ for hyphenated words and word groups, and $\mathbf{W}$ for wider window.

The experiments were spliced in two sets. The first set does not include the feature Dictionary, while the second set does. Each of the contextual predicate combinations were evaluated using different cutoff values: 0, 2, 4, 8, 16, 32, 64, 128, 256 and 512 .

\begin{tabular}{|c|c|c|c|c|c|c|c|c|c|c|}
\hline 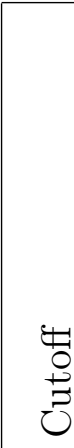 & 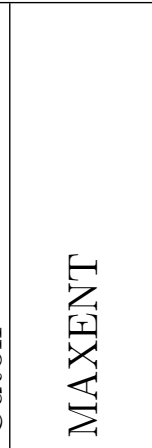 & 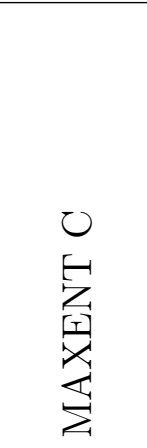 & 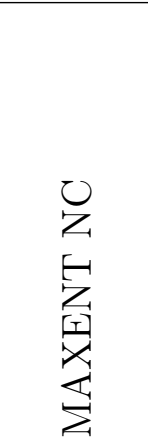 & 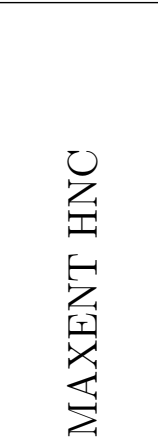 & 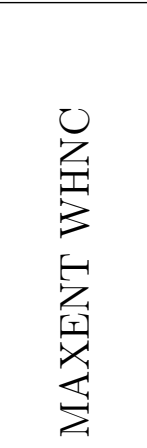 & 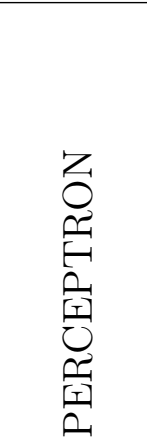 & 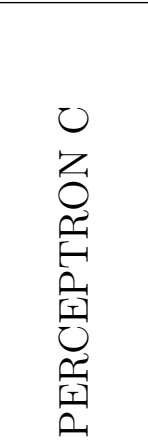 & 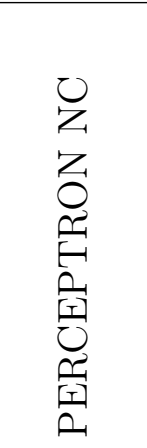 & 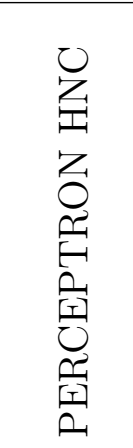 & 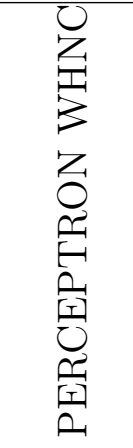 \\
\hline 0 & 95.086 & 94.901 & 94.900 & 4.567 & 4.066 & 6 & 6.77 & 6.708 & 6.82 & 6.831 \\
\hline 2 & 95.558 & 95.429 & 95.428 & 95.266 & 94.664 & 96.463 & 96.519 & 96.488 & 96.575 & 96.679 \\
\hline 4 & 95.532 & 95.393 & 95.393 & 95.218 & 94.754 & 96.272 & 96.306 & 96.332 & 96.372 & 96.489 \\
\hline 8 & 95.512 & 95.382 & 95.374 & 95.297 & 94.845 & 96.074 & 96.130 & 96.111 & 96.215 & 96.282 \\
\hline 16 & 95.449 & 95.323 & 95.323 & 95.318 & 94.873 & 95.921 & 95.953 & 95.940 & 96.118 & 96.125 \\
\hline 32 & 95.358 & 95.256 & 95.264 & 95.256 & 94.787 & 95.740 & 95.724 & 95.751 & 95.910 & 95.857 \\
\hline 64 & 95.126 & 94.954 & 94.974 & 95.054 & 94.552 & 95.446 & 95.480 & 95.470 & 95.571 & 95.552 \\
\hline 128 & 94.598 & 94.454 & 94.472 & 94.517 & 94.065 & 94.983 & 95.050 & 95.023 & 95.268 & 95.157 \\
\hline 256 & 93.983 & 93.858 & 93.909 & 93.832 & 93.325 & 94.578 & 94.648 & 94.593 & 94.743 & 94.561 \\
\hline 512 & 92.671 & 92.585 & 92.608 & 92.644 & 92.218 & 93.720 & 93.695 & 93.780 & 93.918 & 93.808 \\
\hline
\end{tabular}

Table 6.34: 10-fold cross validation results for different configurations of the Featurizer. No dictionary in the sequence validator. The highest accuracy of each configuration is in bold, and the overall highest score is underlined.

Without feature dictionary The highest accuracy score was $96.831 \%$ obtained by a Perceptron model, cutoff zero, with all additional contextual predicates activated. Table 6.34 summarizes the experiment results. Maxent models performed worse than any Perceptron model and suffered from adding more context. The best Maxent model was the one with 

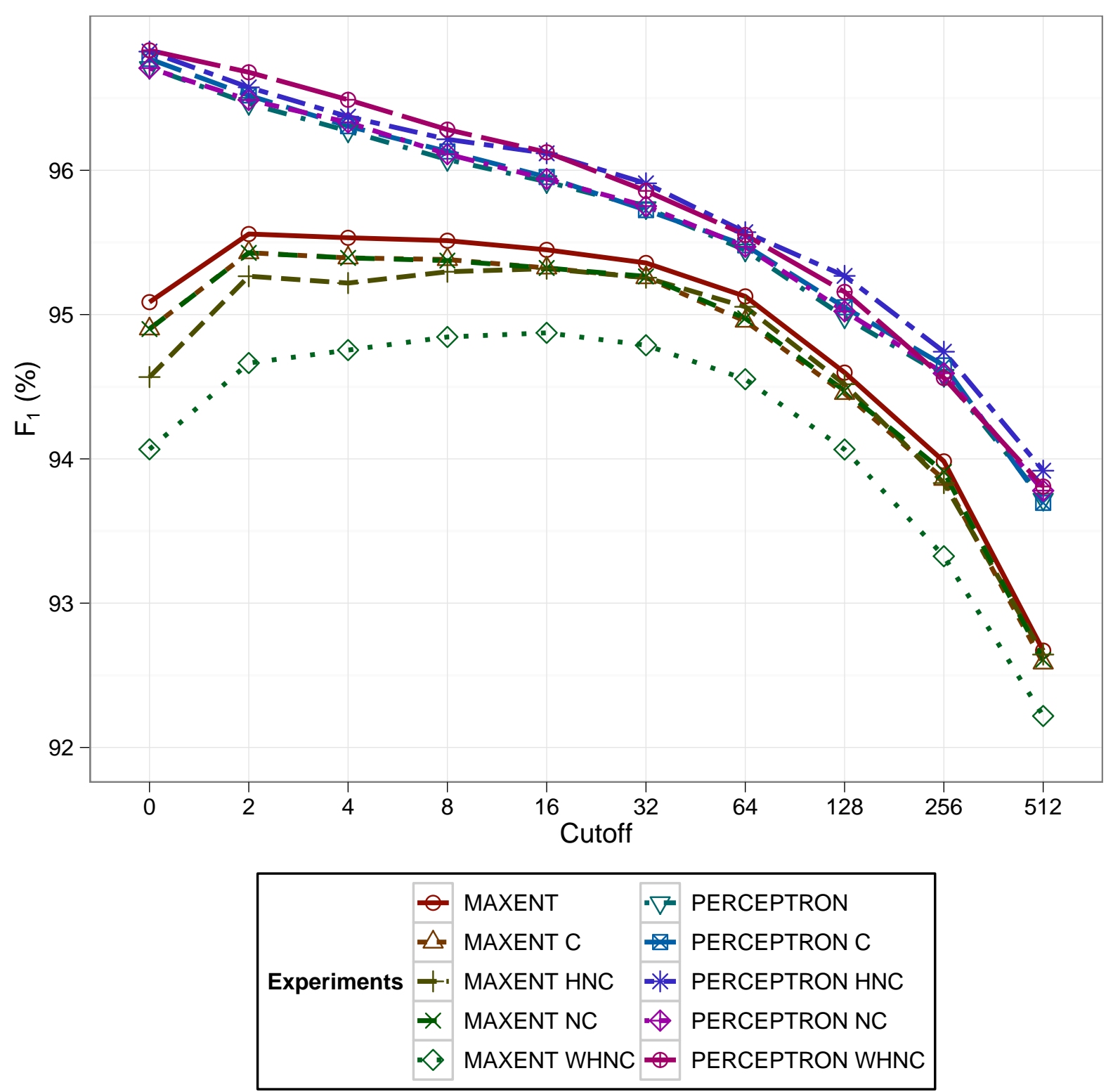

Figure 6.9: Distribution of the accuracy according to the Featurizer configuration and the cutoff. No dictionary in the sequence validator.

a default context only. Perceptron models performed a lot better, and the more context is added, the better the accuracy is.

Figure 6.9 shows how the accuracy scores are distributed according to the configuration and the cutoff. Perceptron models benefit from zero cutoff, while Maxent models have higher accuracy for cutoff equals 2 . The figure also shows that the accuracy values of models trained with the same algorithm are very close to each other.

With feature dictionary With the feature dictionary the highest accuracy score was $96.743 \%$ obtained again by a Perceptron model, cutoff zero, with all additional contextual predicates activated. The value is almost $0.09 \%$ lower than the one scored without the feature 
dictionary. This should be explained by divergences between the dictionary and the training corpus. Table 6.35 summarizes the experiment results.

Table 6.35 and Figure 6.10 show how the scores are very similar to each other. In fact most of the lexemes are covered by the feature dictionary and, because it is used in the sequence validator, the classification from the dictionary prevails.

\begin{tabular}{|c|c|c|c|c|c|c|c|c|c|c|}
\hline 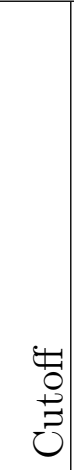 & $\begin{array}{l}\text { 学 } \\
\text { 吝 } \\
\text { 足 }\end{array}$ & 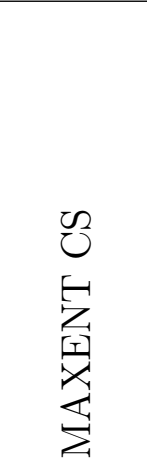 & 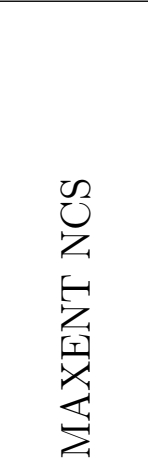 & $\begin{array}{l}\text { 己 } \\
\text { 至 } \\
\text { 尝 } \\
\text { 公 } \\
\text { 岁 }\end{array}$ & 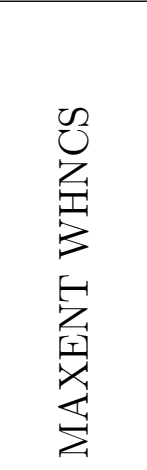 & 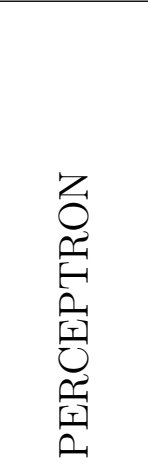 & 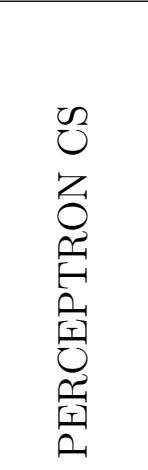 & 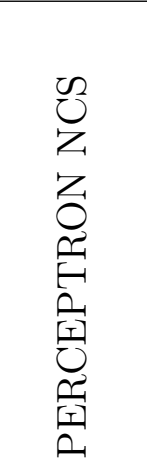 & 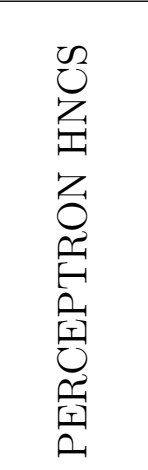 & 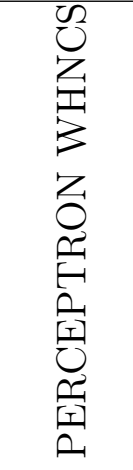 \\
\hline 0 & 96.575 & 96.565 & 96.567 & 96.581 & 96.586 & 96.621 & 96.659 & 96.620 & 96.703 & 96.743 \\
\hline 2 & 96.602 & 96.601 & 96.610 & 96.638 & 96.637 & 96.458 & 96.503 & 96.491 & 96.514 & 96.691 \\
\hline 4 & 96.519 & 96.495 & 96.500 & 96.567 & 96.593 & 96.359 & 96.380 & 96.364 & 96.383 & 96.525 \\
\hline 8 & 96.496 & 96.488 & 96.486 & 96.585 & 96.570 & 96.242 & 96.312 & 96.286 & 96.316 & 96.432 \\
\hline 16 & 96.454 & 96.432 & 96.439 & 96.556 & 96.546 & 96.175 & 96.199 & 96.195 & 96.314 & 96.402 \\
\hline 32 & 96.419 & 96.409 & 96.419 & 96.526 & 96.541 & 96.163 & 96.155 & 96.185 & 96.257 & 96.286 \\
\hline 64 & 96.347 & 96.337 & 96.347 & 96.476 & 96.418 & 96.104 & 96.126 & 96.113 & 96.206 & 96.209 \\
\hline 128 & 96.246 & 96.242 & 96.256 & 96.372 & 96.318 & 96.058 & 96.040 & 96.042 & 96.180 & 96.138 \\
\hline 256 & 96.098 & 96.164 & 96.190 & 96.246 & 96.159 & 95.897 & 95.908 & 95.915 & 96.048 & 95.996 \\
\hline 512 & 95.861 & 95.993 & 96.027 & 96.090 & 96.038 & 95.748 & 95.709 & 95.748 & 95.887 & 95.908 \\
\hline
\end{tabular}

Table 6.35: 10-fold cross validation results for different configurations of the Featurizer with the feature dictionary in the sequence validator. The highest accuracy of each configuration is in bold, and the overall highest score is underlined.

\subsubsection{Previous Work}

Silva (2007) describes a component called Nominal Featurizer, which focuses on number and gender. The component takes advantage of the morphology regularity of inflections and apply a set of rules to recognize features. Exceptions are handled by a dictionary. The reported accuracy is $94.81 \%$.

\subsubsection{Evaluating the Grammar Checker}

This evaluation consists of replacing the Featurizer module of BASELINE by those proposed here and verifying the grammar checker effectiveness using annotated corpora. BASELINE is the CoGrOO 3.1.2 with the new sentence boundary detector, tokenizer, proper name finder, contraction finder and pos tagger, proposed in the previous sections. 

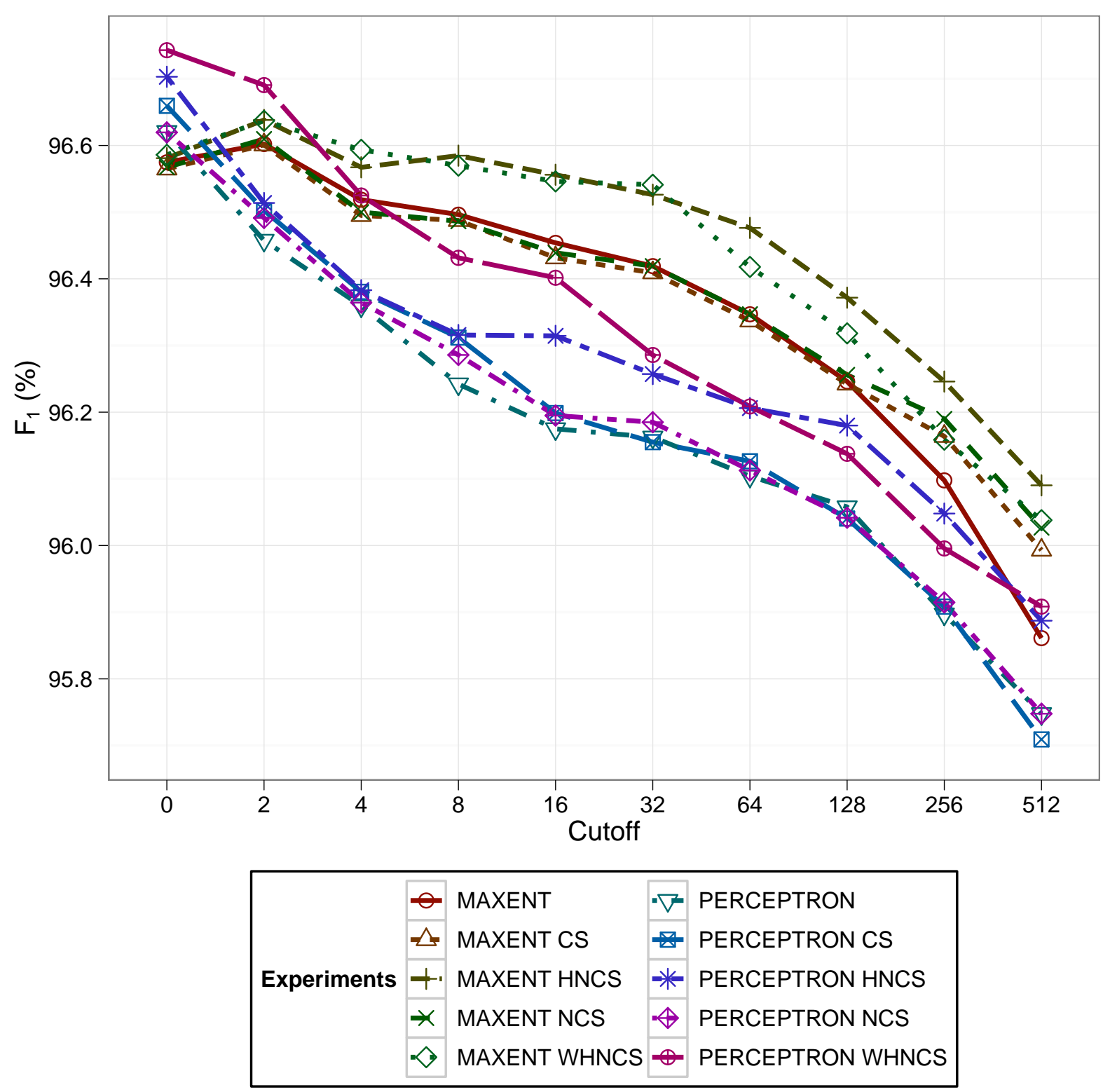

Figure 6.10: Distribution of the accuracy according to the Featurizer configuration and the cutoff. These experiments includes the feature dictionary in the sequence validator.

As example of the pos tagger, the Chunker and Shallow Parser were disabled in this evaluation, and so the phrase-local and subject-verb rules are not evaluated.

\section{Metro Corpus}

In the Metro corpus, the Featurizer with dictionaries did not change the number of true positives compared to the experiments with the new POS Tagger, which removed a true positive, the sentence MT40-8. The Featurizer without dictionary on the other hand reduced the number of true positives in this corpus. This is the case of 0 circuito da vacas (...) (The cow path), in MT28-15, both BASELINE and the Featurizer with dictionary caught number discrepancies between the lexemes da and vacas, the correct 


\begin{tabular}{|c|c|c|c|c|c|c|c|}
\hline \multirow[b]{2}{*}{ Experiment } & \multicolumn{3}{|c|}{ Metro } & \multicolumn{3}{|c|}{ Probi } & \multirow{2}{*}{$\begin{array}{c}\text { Bosque } \\
\text { FP }\end{array}$} \\
\hline & Target & $\mathrm{TP}$ & $\mathrm{FP}$ & Target & $\mathrm{TP}$ & $\mathrm{FP}$ & \\
\hline \multirow[t]{2}{*}{ BASELINE } & \multirow{28}{*}{53} & 16 & 6 & \multirow{28}{*}{2616} & 233 & 204 & $\overline{100}$ \\
\hline & & 15 & 6 & & 260 & 139 & 49 \\
\hline \multirow{2}{*}{ PERCEPTRON DIC S } & & & +3 & & +50 & +48 & +10 \\
\hline & & -1 & -3 & & -23 & -112 & -61 \\
\hline \multirow{3}{*}{ PERCEPTRON DIC WHNCS } & & 15 & 6 & & 260 & 132 & $\overline{47}$ \\
\hline & & & +3 & & +50 & +43 & +9 \\
\hline & & -1 & -3 & & -23 & -114 & -62 \\
\hline \multirow{3}{*}{$\begin{array}{l}\text { PERCEPTRON DIC HNCS } \\
\text { PERCEPTRON DIC NCS }\end{array}$} & & 15 & 6 & & 260 & 138 & 48 \\
\hline & & & +3 & & +50 & +47 & +9 \\
\hline & & -1 & -3 & & -23 & -112 & -61 \\
\hline \multirow{3}{*}{ PERCEPTRON DIC CS } & & 15 & 6 & & 260 & 137 & 48 \\
\hline & & & +3 & & +50 & +46 & +9 \\
\hline & & -1 & -3 & & -23 & -112 & -61 \\
\hline \multirow{3}{*}{ PERCEPTRON WHNCS } & & 13 & 6 & & 204 & 109 & 27 \\
\hline & & & +3 & & +27 & +35 & +4 \\
\hline & & -3 & -3 & & -56 & -129 & -77 \\
\hline \multirow{3}{*}{ PERCEPTRON HNCS } & & 13 & 6 & & 219 & 118 & 29 \\
\hline & & & +3 & & +39 & +39 & +4 \\
\hline & & -3 & -3 & & -53 & -124 & -75 \\
\hline \multirow{3}{*}{ PERCEPTRON CS } & & 13 & 5 & & 204 & 113 & 29 \\
\hline & & & +2 & & +27 & +37 & +4 \\
\hline & & -3 & -3 & & -56 & -127 & -75 \\
\hline \multirow{3}{*}{ PERCEPTRON S } & & 13 & 5 & & 214 & 115 & 31 \\
\hline & & & +2 & & +34 & +39 & +6 \\
\hline & & -3 & -3 & & -53 & -127 & -75 \\
\hline \multirow{3}{*}{ PERCEPTRON NCS } & & 13 & 5 & & 204 & 113 & 29 \\
\hline & & & +3 & & +27 & +37 & +4 \\
\hline & & -3 & -4 & & -56 & -127 & -75 \\
\hline
\end{tabular}

Table 6.36: Summary of the grammar checker evaluation using different configuration of the featurizer. The number in the experiment name refers to the cutoff value used. Bosque corpus does not include grammar error annotations. The entries are grouped by the presence or not of the dictionary, also the PERCEPTRON DIC S, used in the evaluation of the POS Tagger, was placed in the second row, next to the BASELINE. Signed numbers refer to removed (when negative) or added (when positive) entries.

would be das. Without the dictionary the lexeme vacas was classified as singular instead of plural, as the dictionary would prescribe.

A similar effect with the number of false positives, without the dictionary, the featurizer became more flexible and used the context to classify words. This is the case of (...) percorrer o restante do trecho a pé. (go through the rest of the stretch on foot.) in sentence MT23-17. The POS Tagger wrongly classified the lexeme a as an article instead of preposition, and this caused a false positive if the featurizer has a dictionary, but without it, the featurizer classified the masculine noun pé as feminine because of the 
context, and the grammar checker did not catch the false positive again.

In this corpus, varying contextual predicates keeping the dictionary had no effects; all configurations obtained the same number of true positives and false positives.

\section{Probi Corpus}

As described in the experiments with the Metro corpus, the dictionary had a positive effect in the grammar checker. Table 6.37 shows how the dictionary affects the precision and recall; the recall especially suffers from working without the dictionary, certainly because the featurizer grows more flexible and adapts itself to agreement mistakes.

Considering only models that include the dictionary, they all received the exact same number of true positives. The difference lies only in the results for false positives, in which the model PERCEPTRON DIC WHNCS 0 performs the best. The wider context made it easier to guess the correct feature tag for unknown words, like in o Maracanã (the Maracanã). With a smaller context the lexeme Maracanã, which refers to a Brazilian stadium, was classified as a feminine noun, but in fact it is a masculine noun. All the other configuration made small changes in the number of false positives.

\begin{tabular}{|l|r|r|r|}
\hline \multicolumn{1}{|c|}{ Experiment } & Precision & Recall & \multicolumn{1}{c|}{$F_{1}$} \\
\hline \hline BASELINE & 53.32 & 8.91 & 15.26 \\
\hline PERCEPTRON DIC S & 65.16 & $\mathbf{9 . 9 4}$ & 17.25 \\
\hline PERCEPTRON DIC WHNCS & $\mathbf{6 6 . 3 3}$ & $\mathbf{9 . 9 4}$ & $\mathbf{1 7 . 2 9}$ \\
\hline PERCEPTRON DIC HNCS & 65.33 & $\mathbf{9 . 9 4}$ & 17.25 \\
\hline PERCEPTRON DIC NCS & 65.33 & $\mathbf{9 . 9 4}$ & 17.25 \\
\hline PERCEPTRON DIC CS & 65.49 & $\mathbf{9 . 9 4}$ & 17.26 \\
\hline PERCEPTRON WHNCS & 65.18 & 7.80 & 13.93 \\
\hline PERCEPTRON HNCS & 64.99 & 8.37 & 14.83 \\
\hline PERCEPTRON CS & 64.35 & 7.80 & 13.91 \\
\hline PERCEPTRON S & 65.05 & 8.18 & 14.53 \\
\hline PERCEPTRON NCS & 64.35 & 7.80 & 13.91 \\
\hline
\end{tabular}

Table 6.37: Summary of the grammar checker evaluation against Probi corpus varying the featurizer configuration. The maximun value for precision, recall and in bold.

\section{Bosque Corpus}

The results for Bosque corpus reflects the analysis made for the two other corpus.

\subsubsection{Conclusion}

All featurizer perceptron models obtained better $F_{1}$ score compared to similar maxent models in the 10-fold cross-validation experiments, so the perceptron model proved better for this task. Also, the addition of predicates helped raise the model score for perceptron 
models. The presence of the feature dictionary reduced the $F_{1}$, and maybe this is due to divergences of classification of words in the dictionary and in the corpus.

On the other hand, the dictionary proved important for the proofreading task. Both the precision and recall of the grammar checker improved by using models with dictionaries. The addition of predicates also reflected in the improvement of these scores.

In the 10-fold cross-validation, the Perceptron model obtained better $F_{1}$ score than Maxent: 96.291 against 96.137. Customizing the context generator for Portuguese proved really effective, also a good quality dictionary contributed substantially.

To choose the best configuration considering the results from the grammar checker evaluation is quite challenging. The precision of Perceptron models are higher than Maxent models because of the lower number of false positives, while the recall goes in the opposite direction, because Maxent models provide more true positives. The $F_{1}$ combines precision and recall in only one score, and it would be fair to use the model with the best $F_{1}$. But it is important for a grammar checker to have the lowest number of false positives as possible, and Perceptron models performed much better than Maxent models.

Therefore, considering the experimental results the best featurizer model is:

- Algorithm: Perceptron

- Feature dicionary: yes

- Additional features (token class, number, hyphenated and groups): yes

- Wider window: yes

- Cutoff: zero

The new Featurizer will be incorporated to BASELINE and used during the evaluation of the other modules. 


\subsection{Chunker}

Text chunking consists of dividing a text into a syntactically correlated groups of words, like noun and verb phrases, without specifying their internal structure or their role in the main sentence. Chunking is necessary in grammar checking for a few reasons. Firstly, it groups noun phrases, making it easier to check its internal agreement (articles, nouns and adjectives). Secondly, it is a step before shallow parsing, which allows checking subject verb agreement for example.

The sentences in the Bosque corpus, used to train and evaluate the module, are represented in a deep tree structure. A heuristic had to be applied to these sentence trees to extract chunks. The heuristic was proposed by (Fernandes et al., 2010), and consists of considering as chunk all consecutive tokens within the same deepest-level phrase. An OpenNLP formatter was implemented to transform these extracted chunks to the IOB2 tagging style used to train OpenNLP models.

The types of chunk phrases are: nominal (np), verbal (vp), prepositional (pp) and adverbial (advp).

Also, for the Rule application module to work, it is important to know the head of the chunk. For example, in the nominal chunk a mulher (the woman), the head would be mulher (woman). The Bosque corpus already annotated chunks with heads, so it was easy to train a machine learning model to perform this task. The Chunker Head Finder was adapted from the standard Chunker by only changing the tag set.

The Chunker module works as follows:

- The module receives a sequence of tokens annotated with morphological tags;

- The context is analyzed and the tokens are grouped by phrases.

And the Chunker Head Finder works as follows:

- The module receives a sequence of tokens annotated with morphological tags, grouped by chunks;

- The context is analyzed and for each chunk a head is determined.

\subsubsection{Contextual Predicates}

The development of the Chunker module requires the selection of the contextual templates. The contextual predicates are the combination of the contextual template and the actual data. The contextual predicates for chunking were initially proposed by Sha and Pereira (2003) and consist of extracting from a window of size five, including the lexeme under evaluation, the two previous and the two next lexemes, features like the following:

- the lexeme; 
- the POS tags;

- the outcomes already selected for the previous tokens; and

- the combination of the these predicates.

The Chunker Head Finder module also includes the output of the Chunker as contextual predicates.

\section{Experiments}

A set of 10-fold cross-validation experiments were carried out using the proposed contextual predicates. Each experiment checks how the Chunker performs for each algorithm, Perceptron and Maxent. Each algorithm was evaluated using different cutoff values: 0, 2, 4, $8,16,32,64,128,256$ and 512 .

\begin{tabular}{|c|c|c|}
\hline $\begin{array}{l}\stackrel{\uplus}{0} \\
\stackrel{+}{=} \\
0\end{array}$ & $\begin{array}{l}\text { 学 } \\
\text { 爻 } \\
\text { 岁 }\end{array}$ & 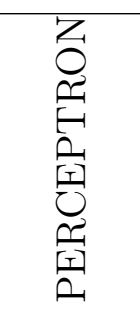 \\
\hline 0 & 95.650 & 96.386 \\
\hline 2 & 95.912 & 96.094 \\
\hline 4 & 95.864 & 95.892 \\
\hline 8 & 95.732 & 95.245 \\
\hline 16 & 95.448 & 95.028 \\
\hline 32 & 95.112 & 94.720 \\
\hline 64 & 94.615 & 94.381 \\
\hline 128 & 93.750 & 94.027 \\
\hline 256 & 93.233 & 93.622 \\
\hline 512 & 91.942 & 93.191 \\
\hline
\end{tabular}

Table 6.38: 10-fold cross validation results for Perceptron and Maxent applied to text chunking. The highest $F_{1}$ of each algorithm is in bold, and the overall highest score is underlined.

The highest $F_{1}$ score was $96.386 \%$ obtained by a Perceptron model with cutoff zero. Table 6.38 summarizes the experiment results. Perceptron proved better than Maxent for this task. Figure 6.11 shows how the accuracy scores are distributed according to the configuration and the cutoff. Perceptron models benefit from zero cutoff, while Maxent models have higher accuracy for cutoff equals 2 .

Table 6.39 shows the effectiveness of the best chunker configuration for each phrase type. Noun phrases, which are the most important for grammar checking, obtained a high $F_{1}$ score: $96.62 \%$.

A second set of 10 -fold cross-validation experiments were carried out to evaluate the Chunker Head Finder, using the proposed contextual predicates. Each experiment checks 


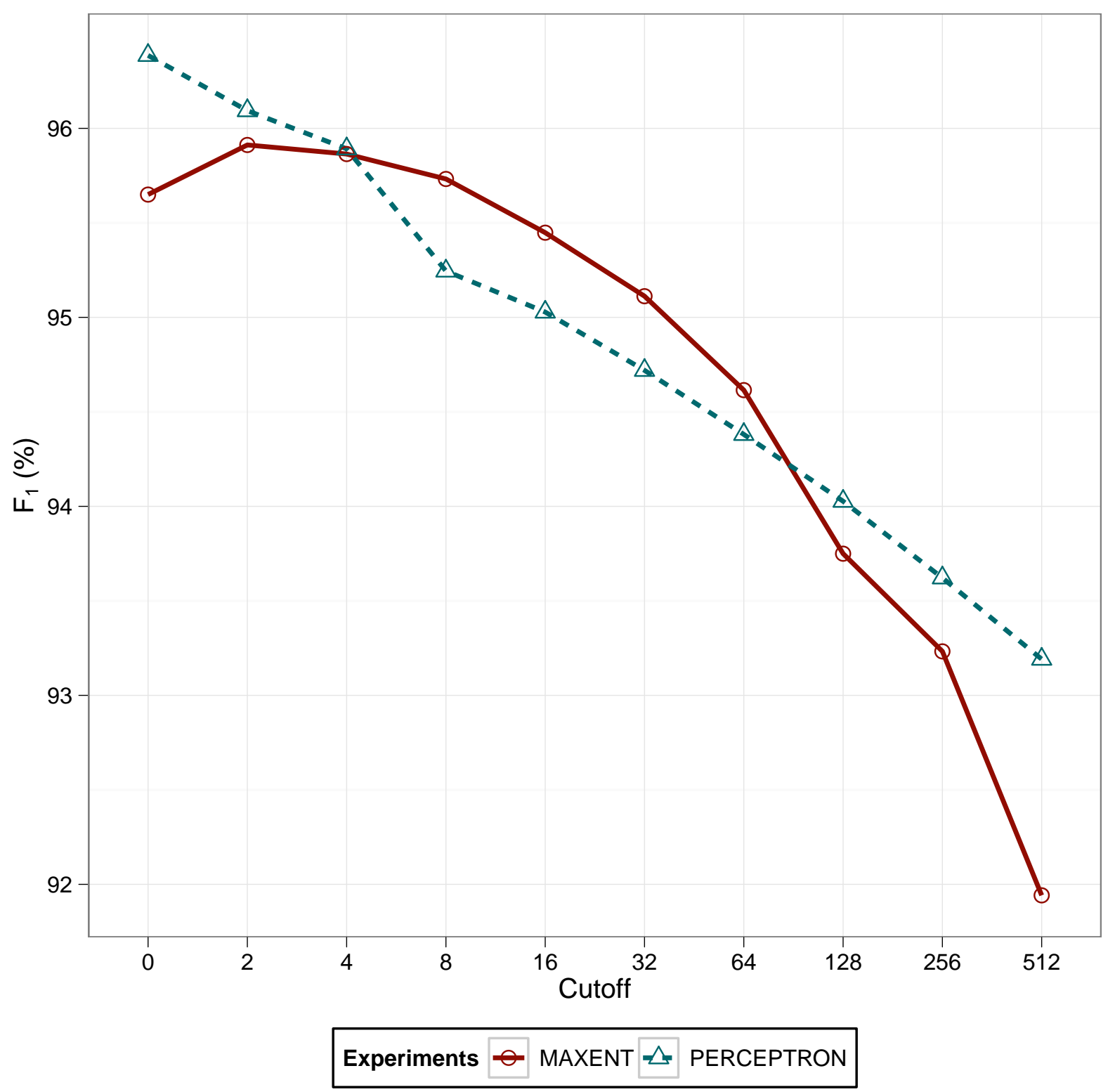

Figure 6.11: Distribution of the $F_{1}$ score according to the algorithm and cutoff.

\begin{tabular}{|l|l|l|l|l|l|l|}
\hline Chunk & Precision & Recall & $F_{1}$ & Target & TP & FP \\
\hline PP & $98.41 \%$ & $98.54 \%$ & $98.47 \%$ & 11976 & 11801 & 191 \\
NP & $96.69 \%$ & $96.55 \%$ & $96.62 \%$ & 24604 & 23755 & 814 \\
VP & $94.77 \%$ & $94.91 \%$ & $94.84 \%$ & 8249 & 7829 & 432 \\
ADVP & $87.82 \%$ & $89.74 \%$ & $88.77 \%$ & 2329 & 2090 & 290 \\
\hline
\end{tabular}

Table 6.39: The chunker effectiveness for each chunk type. The evaluation was performed with a Perceptron model, cutoff 0 .

how the module performs for each algorithm, Perceptron and Maxent. Each algorithm was evaluated using different cutoff values: 0, 2, 4, 8, 16, 32, 64, 128, 256 and 512.

The highest $F_{1}$ score was $99.758 \%$ obtained by a Perceptron model with cutoff two. Table 


\begin{tabular}{|c|c|c|}
\hline $\begin{array}{l}\stackrel{\uplus}{\circ} \\
\stackrel{+}{=} \\
\Xi\end{array}$ & 点 & 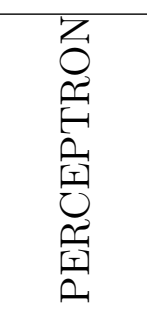 \\
\hline 0 & 99.602 & 99.777 \\
\hline 2 & 99.654 & $\overline{99.775}$ \\
\hline 4 & 99.687 & 99.760 \\
\hline 8 & 99.692 & 99.740 \\
\hline 16 & 99.638 & 99.732 \\
\hline 32 & 99.612 & 99.726 \\
\hline 64 & 99.617 & 99.718 \\
\hline 128 & 99.555 & 99.700 \\
\hline 256 & 99.409 & 99.570 \\
\hline 512 & 99.318 & 99.521 \\
\hline
\end{tabular}

Table 6.40: 10-fold cross validation results for Perceptron and Maxent applied finding the head of a chunk. The highest $F_{1}$ of each algorithm is in bold, and the overall highest score is underlined.

6.40 summarizes the experiment results. Once again Perceptron proved better than Maxent. Table 6.41 shows an interesting aspect of the head finder, $69.36 \%$ of the chunks has length one, which consists of the trivial case. The high effectiveness holds even for longer chunks.

\begin{tabular}{|l|l|l|l|l|l|l|}
\hline Length & Precision & Recall & $F_{1}$ & Target & $\mathrm{TP}$ & FP \\
\hline 1 & $100.00 \%$ & $100.00 \%$ & $100.00 \%$ & 31179 & 31179 & 0 \\
2 & $99.72 \%$ & $99.72 \%$ & $99.72 \%$ & 12770 & 12734 & 36 \\
3 & $98.10 \%$ & $98.10 \%$ & $98.10 \%$ & 2740 & 2688 & 52 \\
4 & $94.64 \%$ & $94.64 \%$ & $94.64 \%$ & 392 & 371 & 21 \\
5 & $89.09 \%$ & $89.09 \%$ & $89.09 \%$ & 55 & 49 & 6 \\
6 & $93.75 \%$ & $93.75 \%$ & $93.75 \%$ & 16 & 15 & 1 \\
7 & $100.00 \%$ & $100.00 \%$ & $100.00 \%$ & 6 & 6 & 0 \\
\hline
\end{tabular}

Table 6.41: Detailed 10-fold cross validation results for Perceptron and Maxent applied finding the head of a chunk. Each row shows how effectiveness of the task of finding the head of a chunk varies according to the chunk size.

\subsubsection{Previous Work}

In addition to the chunker from CoGrOO 3.1.2, the search for another application for Portuguese had no results, as also noted by Fernandes et al. (2010), who implemented a chunker but did not published any evaluation results.

The chunker from CoGrOO 3.1.2 was evaluated using a different metric and it is not easy to compare its effectiveness with the chunker proposed here directly. On the other hand, for the English language there is evaluation information for quite a few systems. For example, 
the CONLL 2000 Shared Task was text chunking (Sang and Buchholz, 2000), and the best system $F_{1}$ was $94.13 \%$. Later, Sha and Pereira (2003) proposed another English chunker that could achieved a $F_{1}$ score of $94.39 \%$ for the same training data.

\subsubsection{Evaluating the Grammar Checker}

This evaluation consists of replacing the Chunker module of BASELINE by the Chunker + Head Finder proposed here and verifying the grammar checker effectiveness using annotated corpora. BASELINE is the CoGrOO 3.1.2 with the new sentence boundary detector, tokenizer, proper name finder, contraction finder and pos tagger, proposed in the previous sections.

As example of the pos tagger, the Shallow Parser was disabled in this evaluation. To make it easier, only rules affected by the Chunker and those that do not require the Shallow Parser to be enabled, i.e. only the phrase-local rules will be evaluated.

The head information is not used by any of these rules, so changing its configuration does not change the effectiveness results in these experiments. The chunk head information is important for rules that also depend on the shallow parser, and the impact of the changes will be evaluated in the shallow parser section.

\begin{tabular}{|c|c|c|c|c|c|c|c|}
\hline \multirow{3}{*}{ Experiment } & \multirow{2}{*}{\multicolumn{3}{|c|}{ Metro }} & & & & \\
\hline & & & & \multicolumn{3}{|c|}{ Probi } & \multirow{2}{*}{$\begin{array}{c}\text { Bosque } \\
\text { FP }\end{array}$} \\
\hline & Target & $\mathrm{TP}$ & $\mathrm{FP}$ & Target & $\mathrm{TP}$ & $\mathrm{FP}$ & \\
\hline BASELINE & \multirow{7}{*}{53} & 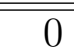 & 10 & \multirow{7}{*}{2616} & 37 & 81 & 29 \\
\hline \multirow{3}{*}{ PERCEPTRON 0} & & 2 & 13 & & 67 & 70 & 26 \\
\hline & & +2 & +5 & & +38 & +29 & +18 \\
\hline & & & -2 & & -8 & -40 & -21 \\
\hline \multirow{3}{*}{ MAXENT 2} & & 2 & 13 & & 68 & 73 & 27 \\
\hline & & +2 & +5 & & +38 & +31 & +18 \\
\hline & & & -2 & & -7 & -39 & -20 \\
\hline
\end{tabular}

Table 6.42: Summary of the grammar checker evaluation using different chunk configurations. The number in the experiment name refer to the cutoff value used. Bosque corpus does not include grammar error annotations. Signed numbers refer to removed (when negative) or added (when positive) entries.

\section{Metro Corpus}

Both Perceptron and Maxent models performed the same in Metro corpus. It included two new true positives and also changed the number of false positives. All changes are related to changes in noun phrases.

The most common cause of false positives is a combination of the poor performance of the chunker in a few constructions and the naive rule implementation for noun and adjective agreement, which simple checks if a noun agrees with the following adjective without a deeper context analysis. For example, the introduced false positive in (...) a localização 
de ruas e de pontos de interesse próximos das estações (...) (location of streets and points of interest near stations) in the sentence MT25-10, in which interesse próximos was grouped as a noun phrase, and since the tokens do not agree in number, caused the error. But in fact próximos (nearby) agrees with pontos (points). All the other false positives are similar to this one.

On the other hand, the chunker was more effective with simple phrases, which is why it obtained two new true positives.

\section{Probi Corpus}

The new chunker caused a noticeable improvement in the grammar checker effectiveness while evaluating with the Probi corpus. The number of true positives increased as well as the number of false positives decreased. The differences between the effectiveness while using Perceptron or Maxent model are not relevant. The first obtained slightly higher precision, while the latter, a little better in recall.

The analysis done for Metro corpus holds for Probi corpus. The new chunker performed better for simple chunks, but often failed for those with higher complexity. Also, as the BASELINE includes the POS Tagger from the CoGrOO 3.1.2, several of the improvements are related to some change in the part-of-speech tags that caused a change in the chunks configuration.

\section{Bosque Corpus}

Again the new chunker proved better by removing some false positives. The previous analysis still holds. Perceptron and Maxent models performed almost the same, the only difference is that the first obtained one less false positive.

\subsubsection{Conclusion}

The chunker trained with Perceptron model obtained better $F_{1}$ scores compared to the Maxent model in the 10-fold cross-validation experiments, so the the Perceptron model proved better for this task. Additional predicates were not evaluated here, but should improve the effectiveness. This is left for future studies.

Also, the grammar checker effectiveness improved with both Perceptron and Maxent models, and the difference between each model in the grammar checker evaluation was negligible.

Considering this experimental results, especially the 10-fold cross-validation experiments, the best chunker model is:

- Algorithm: Perceptron

- Cutoff: zero 
The new Chunker will be incorporated to BASELINE and used during the evaluation of the other modules. 


\subsection{Shallow Parser}

The purpose of the Shallow Parser is to detect the subject (a noun phrase) and a verb (a verbal phrase) of a sentence, as well as predicative clauses and verb objects. This information is used later by the rule module to verify verb agreement.

The sentences in the Bosque corpus, used to train and evaluate the module, are represented in a deep tree structure. As was done with the chunker, a heuristic had to be applied to these sentence trees to extract shallow syntactic structures, called from now on syntactic chunk. The heuristic was proposed by (Fernandes et al., 2010), and consists of considering as a syntactic chunk all consecutive tokens within the same deepest-level phrase. An OpenNLP formatter was implemented to transform these extracted chunks to the IOB2 tagging style used to train OpenNLP models.

The types of syntactic chunk phrases considered are: subject (subj), direct object (acc), indirect object with preposition (dat), prepositional object (piv), subject predicate (sc), object predicate $(\mathbf{o c})$, predicator $(\mathbf{p})$, advebial object (sa), adverbial object (advl) and identifying apposition (app).

The Shallow Parser module works as follows:

- the module receives a sequence of tokens annotated with morphological tags and chunks;

- the context is analyzed and the tokens are grouped by syntactic structure.

\subsubsection{Contextual Predicates}

The development of the Shallow Parser module requires the selection of the contextual templates. The contextual predicates are the combination of the contextual template and the actual data. The contextual predicates are derived from the one proposed for chunking, initially proposed by Sha and Pereira (2003), which consists of extracting from a window of size five, including the lexeme under evaluation, the two previous and the two next lexemes, features like the following:

- the lexeme;

- the POS tags;

- the Chunk tags;

- the outcomes already selected for the previous tokens; and

- the combination of these predicates. 


\section{Experiments}

A set of 10-fold cross-validation experiments were carried out using the proposed contextual predicates. Each experiment checks how the Shallow Parser performs for each algorithm, Perceptron and Maxent. Each algorithm was evaluated using different cutoff values: 0, 2, 4, $8,16,32,64,128,256$ and 512 .

\begin{tabular}{|c|c|c|}
\hline $\begin{array}{l}\text { 岁 } \\
= \\
= \\
=\end{array}$ & $\begin{array}{l}\text { 䒚 } \\
\text { 妾 } \\
\text { 至 }\end{array}$ & 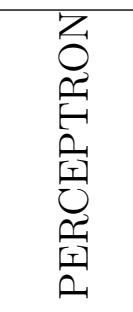 \\
\hline 0 & 81.089 & $\underline{83.150}$ \\
\hline 2 & 81.625 & 82.531 \\
\hline 4 & 81.932 & 81.991 \\
\hline 8 & 81.925 & 81.467 \\
\hline 16 & 81.727 & 80.720 \\
\hline 32 & 81.423 & 80.153 \\
\hline 64 & 80.744 & 79.294 \\
\hline 128 & 80.040 & 78.072 \\
\hline 256 & 79.492 & 75.731 \\
\hline 512 & 77.918 & 74.316 \\
\hline
\end{tabular}

Table 6.43: 10-fold cross validation results for Perceptron and Maxent applied to shallow parsing. The highest $F_{1}$ of each algorithm is in bold, and the overall highest score is underlined.

The highest $F_{1}$ score was $83.150 \%$ obtained by a Perceptron model with cutoff zero. Table 6.43 summarizes the experiment results. Perceptron proved better than Maxent for this task. Figure 6.12 shows how the accuracy scores are distributed according to the configuration and the cutoff. Perceptron models benefit from zero cutoff, while Maxent models have higher accuracy for cutoff equals 4 .

Table 6.44 shows the effectiveness of the best chunker configuration for each syntactic chunk..

\subsubsection{The Multiple Clause Issue}

The main issue with this approach appears while analyzing sentences with multiple clauses. For example, the sentence A mulher que eu conheci ontem me telefonou. (The woman I met yesterday called me.). The sentence subject has an inner relative clause. Nested patterns like this make it impossible to directly find the shallow structure of the sentence. A solution would be to introduce a clause identification module, as described by Fernandes et al. (2010), and extract the shallow syntactic structures from the identified clauses. 


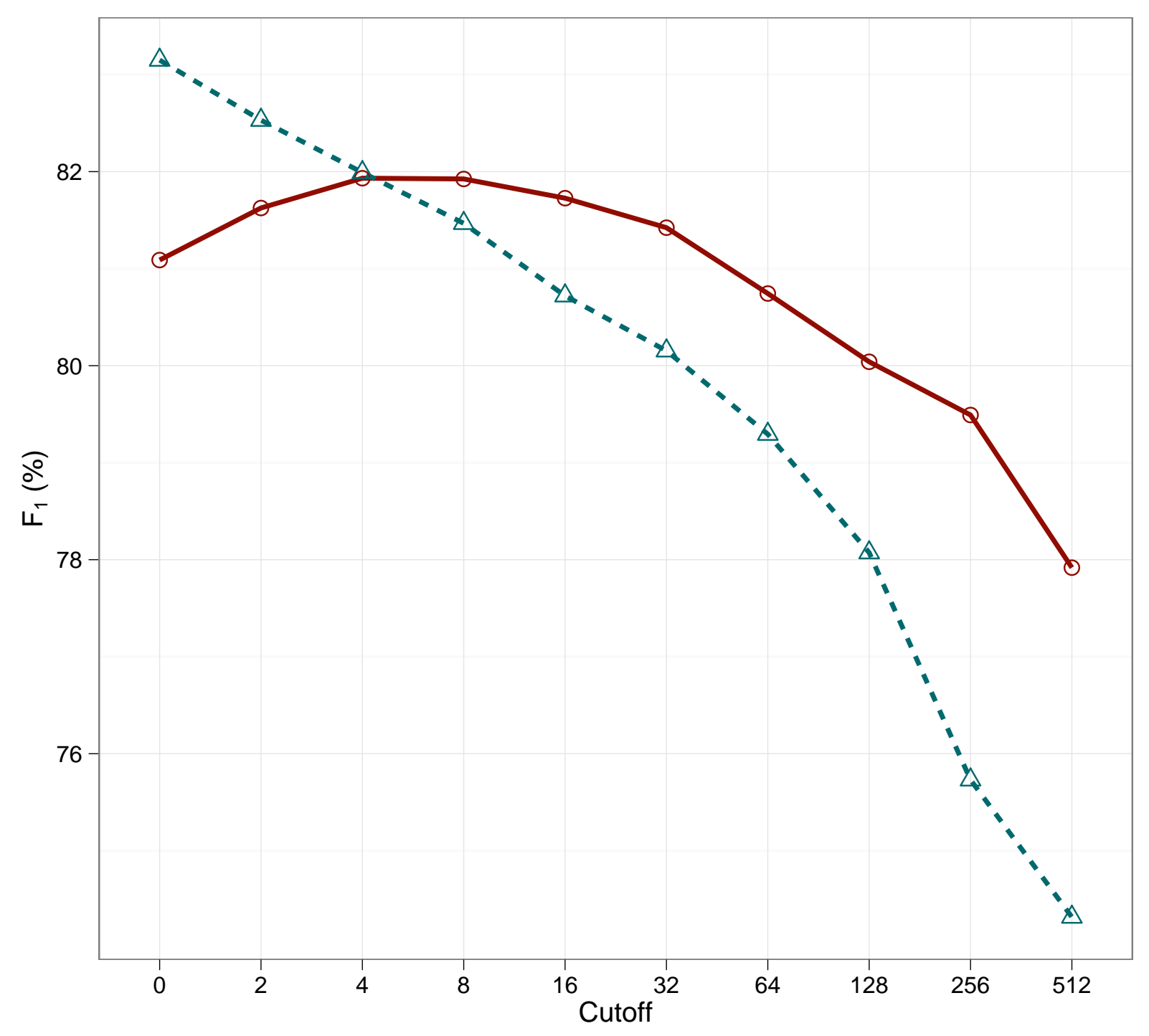

Experiments $\curvearrowright$ MAXENT $\_$PERCEPTRON

Figure 6.12: $F_{1}$ score distribution according to the algorithm and cutoff.

\subsubsection{Gender and Number of Subject and Predicate}

The proofreader rules for subject, verb and predicate agreement requires the code to specify the gender and number of these arguments, but the training corpus does not offer such information for the subject and predicate of sentences. A simplified process was adopted to assign such features to these arguments, they are:

- Each pair prepositional and noun phrases are excluded from the analysis, for example, carro dos sonhos (dream car) would be trimmed to just carro;

- If the argument is composed of only one noun phrase, its gender and number are used;

- If the argument is composed of multiple noun phrases with the pattern $(N P,)^{*} N P e$ 


\begin{tabular}{|l|l|l|l|l|l|l|}
\hline Syntactic Chunk & Precision & Recall & $F_{1}$ & Target & TP & FP \\
\hline P & $99.40 \%$ & $99.78 \%$ & $99.59 \%$ & 8200 & 8182 & 49 \\
SUBJ & $85.83 \%$ & $84.16 \%$ & $84.99 \%$ & 4900 & 4124 & 681 \\
ADVL & $75.78 \%$ & $73.73 \%$ & $74.75 \%$ & 5730 & 4225 & 1350 \\
ACC & $72.25 \%$ & $74.48 \%$ & $73.34 \%$ & 3953 & 2944 & 1131 \\
SC & $74.25 \%$ & $67.75 \%$ & $70.85 \%$ & 1166 & 790 & 274 \\
APP & $75.94 \%$ & $64.84 \%$ & $69.95 \%$ & 219 & 142 & 45 \\
PIV & $56.66 \%$ & $55.14 \%$ & $55.89 \%$ & 1119 & 617 & 472 \\
DAT & $56.25 \%$ & $47.37 \%$ & $51.43 \%$ & 38 & 18 & 14 \\
OC & $57.14 \%$ & $36.36 \%$ & $44.44 \%$ & 99 & 36 & 27 \\
SA & $61.22 \%$ & $30.00 \%$ & $40.27 \%$ & 200 & 60 & 38 \\
\hline
\end{tabular}

Table 6.44: The shallow parser effectiveness for each syntactic chunk type. The evaluation was performed with a Perceptron model, cutoff 0.

$N P$, in other words, optional multiple comma separated noun phrases, a noun phrase followed by the lexeme e (and), and another noun phrase, the number will be plural, and the gender will be feminine if all noun phrases are feminine, or masculine otherwise.

- If the argument does not follow any of the previous rules, the gender and number are marked as unknown.

The rule is quite strict, but works for simple arguments. An improved gender and number for subject and predicate implementation is left as future work.

\subsubsection{Rule Improvements}

The introduction of new tags allowed for more restriction in the rule pattern. For example, rule 124 that checks the agreement between subject and the subject predicate, now can constrain what is a predicate. Before it was only looking for a noun phrase whose head was an adjective, but now it can also check if it is inside a subject predicate.

Although the intention of this work is not to improve the existing rules, it was done especially for subject verb rules because it would dramatically improve the rules if they could use the additional information.

\subsubsection{Evaluating the Grammar Checker}

This evaluation consists of replacing the Shallow Parser module of BASELINE by the Shallow Parser + Chunker Head Finder proposed here and verifying the grammar checker effectiveness using annotated corpora. BASELINE is the CoGrOO 3.1.2 with the new sentence boundary detector, tokenizer, proper name finder, contraction finder, pos tagger, and chunker proposed in the previous sections.

Only rules affected by the Shallow Parser are enabled, i.e. only the subject-verb rules will be evaluated. Table 6.45 shows the experimental results for the different evaluation corpus. 


\begin{tabular}{|c|c|c|c|c|c|c|c|}
\hline & \multicolumn{3}{|c|}{ Metro } & \multicolumn{3}{|c|}{ Probi } & Bosque \\
\hline Experiment & Target & $\mathrm{TP}$ & $\mathrm{FP}$ & Target & $\mathrm{TP}$ & $\mathrm{FP}$ & $\mathrm{FP}$ \\
\hline BASELINE & & 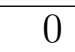 & 10 & & 89 & 378 & 76 \\
\hline $\begin{array}{l}\text { SP PERCEPTRON } 0 \\
\text { HF MAXENT } 8\end{array}$ & & 0 & $\begin{array}{r}3 \\
+2 \\
-9\end{array}$ & & $\begin{array}{r}92 \\
+55 \\
-52\end{array}$ & $\begin{array}{r}74 \\
+48 \\
-352\end{array}$ & $\begin{array}{r}16 \\
+10 \\
-70\end{array}$ \\
\hline $\begin{array}{l}\text { SP PERCEPTRON } 0 \\
\text { HF PERCEPTRON } 0\end{array}$ & 53 & 0 & $\begin{array}{r}3 \\
+2 \\
-9\end{array}$ & 2616 & $\begin{array}{r}92 \\
+55 \\
-52\end{array}$ & $\begin{array}{r}75 \\
+46 \\
-349\end{array}$ & $\begin{array}{r}16 \\
+10 \\
-70\end{array}$ \\
\hline $\begin{array}{l}\text { SP MAXENT } 4 \\
\text { HF MAXENT } 8\end{array}$ & & 0 & $\begin{array}{r}2 \\
+1 \\
-9\end{array}$ & & $\begin{array}{r}91 \\
+54 \\
-52\end{array}$ & $\begin{array}{r}77 \\
+50 \\
-351\end{array}$ & $\begin{array}{r}15 \\
+10 \\
-71\end{array}$ \\
\hline $\begin{array}{l}\text { SP MAXENT } 4 \\
\text { HF PERCEPTRON } 0\end{array}$ & & 0 & $\begin{array}{r}2 \\
+1 \\
-9\end{array}$ & & $\begin{array}{r}91 \\
+54 \\
-52\end{array}$ & $\begin{array}{r}78 \\
+48 \\
-348\end{array}$ & $\begin{array}{r}15 \\
+10 \\
-71\end{array}$ \\
\hline
\end{tabular}

Table 6.45: Summary of the grammar checker evaluation using different Shallow Parser configurations. The number in the experiment name refer to the cutoff value used. Bosque corpus does not include grammar error annotations. Signed numbers refer to removed (when negative) or added (when positive) entries.

\section{Metro Corpus}

The new Shallow Parser and Chunk Head Finder did not change the number of true positives in Metro Corpus, but changed the number of false positives, which number decreased drastically from 10 to 3, for Shallow Parser trained with Perceptron algorithm, and 2 for the one trained with Maxent.

All the removed false positives are related the improved effectiveness of the Shallow Parser and to the new rule constraints. New false positives occur in sentences with multiple clauses, which are not handled properly by the new implementation. This is the case of sentence MT7-8, Example 6.32, which has 77 tokens. The Shallow Parser classified o Largo Santa Cecília e a Praça da República, tudo as the subject of passou, while 
actually it is part of another clause.

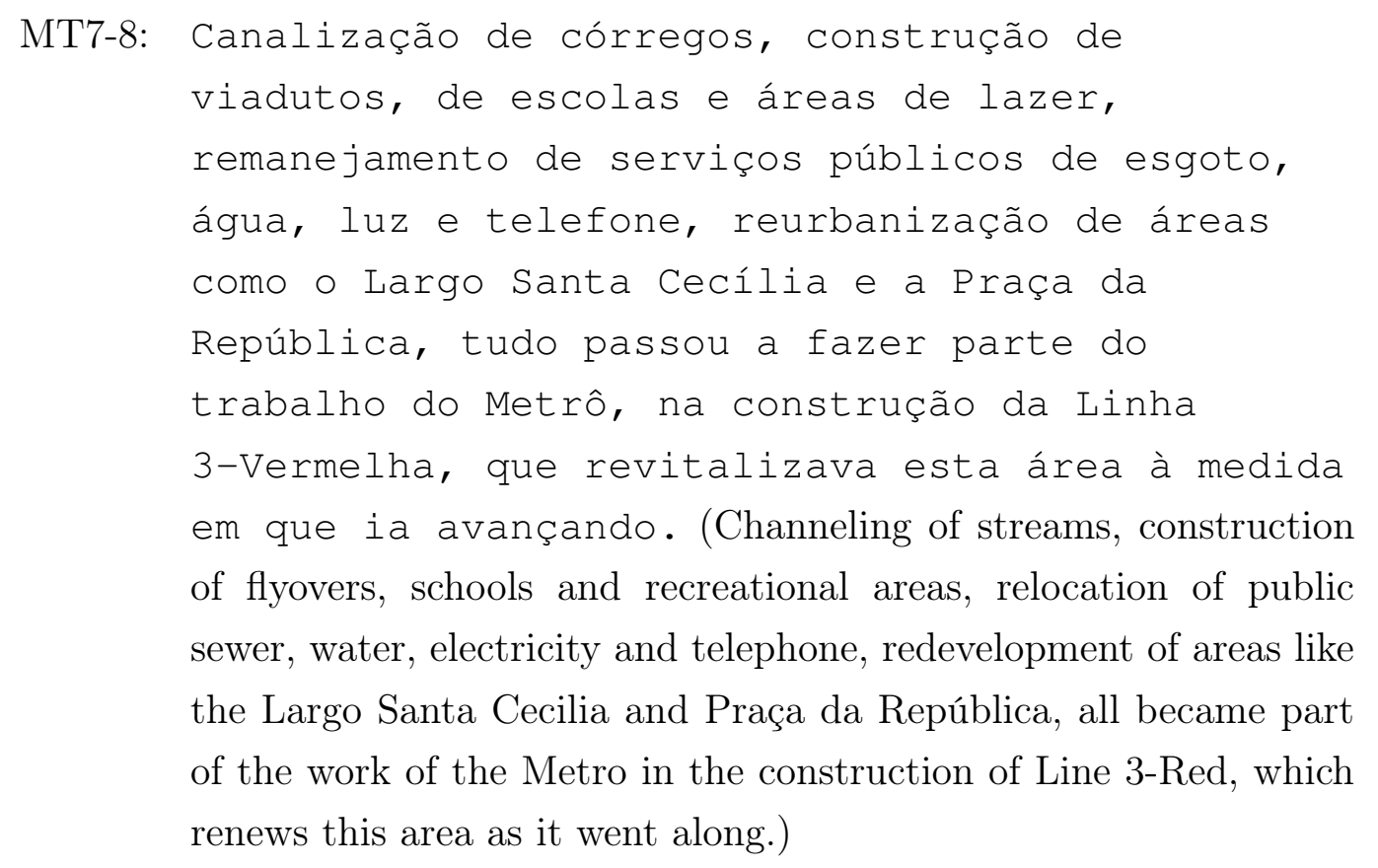

\section{Probi Corpus}

The number of false positives in Probi corpus dropped from 378 to 74, with a Shallow Parser trained with Perceptron and a Chunker Head Finder trained with Maxent. The number of true positives also showed a slight improvement, from 89 to 92 . This means that the precision increased dramatically, from $19.06 \%$ to $55.42 \%$. On the one hand, it still not good because the number of false positives is close to the number of true positive, on the other hand, it is much better than the performance of CoGrOO 3. Table 6.46 shows the complete results.

\begin{tabular}{|l|r|r|r|}
\hline \multicolumn{1}{|c|}{ Experiment } & Precision & Recall & \multicolumn{1}{|c|}{$F_{1}$} \\
\hline \hline BASELINE & 19.058 & 3.402 & 5.774 \\
\hline SP PERCEPTRON 0 + HF MAXENT 8 & $\mathbf{5 5 . 4 2 2}$ & $\mathbf{3 . 5 1 7}$ & $\mathbf{6 . 6 1 4}$ \\
\hline SP PERCEPTRON 0 + HF PERCEPTRON 0 & 55.090 & $\mathbf{3 . 5 1 7}$ & 6.612 \\
\hline SP MAXENT 4 + HF MAXENT 8 & 54.167 & 3.479 & 6.537 \\
\hline SP MAXENT 4 + HF PERCEPTRON 0 & 53.846 & 3.479 & 6.535 \\
\hline
\end{tabular}

Table 6.46: Summary of the grammar checker evaluation against Probi corpus. The maximun value for precision, recall and in bold.

As explained in Metro analysis, the most important source of false positives is multiple clauses in the same sentence., but some of the new false positives are actually related to issues with the evaluation system, or to how the error is classified in the corpus. The sentence 6.33 exemplifies it. There is a disagreement between the subject and the verb, so the grammar 
checker correctly marked it as an error, but the corpus annotated it as a problem in the use of accent mark, which is also correct.
PROBI-ace-92-17: Eles provém a casa do necessário. (They provide what is needed for the house.)
Error: The subject (Eles) is plural, but the verb (provém) is singular. Should be provêm.

Another source of error is some special cases of Portuguese agreement rules. For example in 6.34, where the predicate does not need to agree with the in number with the subject.

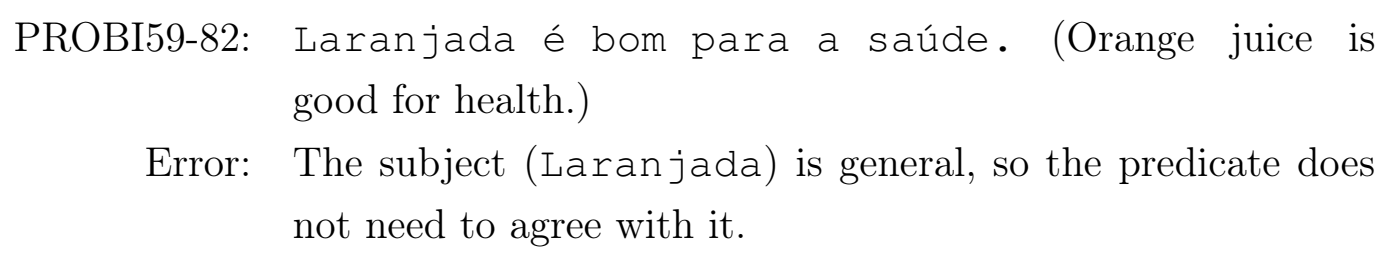

The Chunker Name Finder affected the results. The Maxent model performed better compared to the one trained with Perceptron: it obtained one additional true positive and a reduced number of false positives.

\section{Bosque Corpus}

The number of false positives improved significantly, dropping from 76 to 16 with the Shallow Parser trained with Perceptron model. For this corpus, the Maxent model obtained one less false positive.

Again, the number of false positives is related to multiple clauses and to special cases of Portuguese agreement rules. Example 6.35 shows one of the special cases that caused a false positive. The subject indicates a partition: A maioria dos voluntários (most of the volunteers). The agreement rule for subjects formed by partitive expression states that the verb can be in both singular or plural form. This exception is not taken into account by the Rule application module, causing a false positive.

CF918-3: A maioria dos voluntários são jovens. (Most of the volunteers are young.)

Error: The verb can be both plural or singular if the subject indicates a partition (A maioria dos voluntários), but CoGrOO erroneously indicates an agreement error. 


\subsubsection{Conclusion}

The Perceptron Shallow Parser obtained the highest $F_{1}$ in 10 -fold cross-validation in the evaluation with Probi corpus. The Maxent Shallow Parser obtained one less false positive while evaluating Metro and Bosque compared to Perceptron.

Considering this experimental results, especially the 10-fold cross-validation experiments, the best Shallow Parser model is:

- Algorithm: Perceptron

- Cutoff: zero

These experiments also measured the effectiveness of the Chunker Head Finder. Although the 10-fold cross-validation results from table 6.40 shows a slightly higher $F_{1}$ score for the one trained with Perceptron, the one trained with Maxent proved better combined with the other grammar checker elements in the evaluation with Probi corpus. The best Chunk Head Finder model is:

- Algorithm: Maxent

- Cutoff: eight 


\section{Chapter 7}

\section{Final Evalutation}

A final set of experiments was carried out to measure the effective improvements of the new modules in the proofreading task. It analyzed the results obtained in Section 5 side by side and compared them to those obtained by the new version of the grammar checker. Also, in the end of the chapter, CoGrOO is compared to other grammar checkers available for Brazilian Portuguese.

\subsection{PROBI Corpus}

Table 7.1 shows how the grammar checker improved in terms of number of false positives in the experiments with the PROBI corpus. The new version counted 251 false positives against 1181 in the older version. This is an significant improvement that directly affects the user experience. The total number of true positives also improved, raising from 357 to 377 in the new version, causing a significant improvement in the grammar checker precision, which increased from $23.21 \%$ to $60.03 \%$, and consequently in the $F_{1}$ score, which increased from $17.19 \%$ to $23.24 \%$. For a more effective improvement in the $F_{1}$ score, new rules should be introduced to increase coverage, but it this is beyond of the scope of this work and left for future work.

Individually, most of the categories improved. The most important changes are in the con and cov categories, which are noun and verb agreement, respectively. For the former, the precision increased from $26.78 \%$ to $54.05 \%$, which is still low due to the a issue explained in Section 6.6.9. For the latter, the precision increased from $10.87 \%$ to $54.55 \%$. The complete category description of PROBI can be found in Appendix B.

Table 7.2 shows how the scores would change if the nominal agreement rules related to a were disabled. The precision of the category con increased from $54.05 \%$ to $60.35 \%$ disabling it.

There are only two categories that decreased in precision. One more expressive, for the category cra, which checks the usage of accent stress marker (crase). The number of false positives is the same, but the number of true positives decreased from 97 to 86 . The most im- 
portant cause of this regression is the introduction of multiword expressions. For example, the sentence PROBI-cra-116-45 À partir de amanhã tudo estará terminado. (Beginning tomorrow, everything will be finished.), the tokens $\grave{A}$ partir de were grouped as a MWE, causing a false negative. The correct would be A partir de. A solution to issues like this will be left for future studies. Another regression was for the category aha, which number of false positives increased by one.

\begin{tabular}{|c|c|c|c|c|c|c|c|}
\hline Category & Version & Precision & Recall & $F_{1}$ & Target & $\mathrm{TP}$ & $\mathrm{FP}$ \\
\hline \multirow{2}{*}{ ALL } & 3.1 .2 & $23.21 \%$ & $13.65 \%$ & $17.19 \%$ & \multirow{2}{*}{2616} & 357 & 1181 \\
\hline & 4.0 .0 & $60.03 \%$ & $14.41 \%$ & $23.24 \%$ & & 377 & 251 \\
\hline \multirow{2}{*}{ con } & 3.1 .2 & $26.78 \%$ & $22.22 \%$ & $24.29 \%$ & \multirow{2}{*}{441} & 98 & 268 \\
\hline & 4.0 .0 & $54.05 \%$ & $31.75 \%$ & $40.00 \%$ & & 140 & 119 \\
\hline \multirow{2}{*}{ cra } & 3.1 .2 & $76.98 \%$ & $25.19 \%$ & $37.96 \%$ & \multirow{2}{*}{385} & 97 & 29 \\
\hline & 4.0 .0 & $74.78 \%$ & $22.34 \%$ & $34.40 \%$ & & 86 & 29 \\
\hline \multirow{2}{*}{ cop } & 3.1 .2 & $72.73 \%$ & $17.14 \%$ & $27.75 \%$ & \multirow{2}{*}{140} & 24 & 9 \\
\hline & 4.0 .0 & $71.43 \%$ & $21.43 \%$ & $32.97 \%$ & & 30 & 12 \\
\hline \multirow{2}{*}{ mal } & 3.1 .2 & $66.67 \%$ & $20.00 \%$ & $30.77 \%$ & \multirow{2}{*}{10} & 2 & 1 \\
\hline & 4.0 .0 & $66.67 \%$ & $20.00 \%$ & $30.77 \%$ & & 2 & 1 \\
\hline \multirow{2}{*}{$\mathrm{cov}$} & 3.1 .2 & $10.87 \%$ & $18.51 \%$ & $13.69 \%$ & \multirow{2}{*}{562} & 104 & 853 \\
\hline & 4.0 .0 & $54.55 \%$ & $14.95 \%$ & $23.46 \%$ & & 84 & 70 \\
\hline \multirow{2}{*}{ aha } & 3.1 .2 & $36.36 \%$ & $13.79 \%$ & $20.00 \%$ & \multirow{2}{*}{29} & 4 & 7 \\
\hline & 4.0 .0 & $40.00 \%$ & $13.79 \%$ & $20.51 \%$ & & 4 & 6 \\
\hline \multirow{2}{*}{ pro } & 3.1 .2 & $100.00 \%$ & $11.27 \%$ & $20.25 \%$ & \multirow{2}{*}{71} & 8 & 0 \\
\hline & 4.0 .0 & $100.00 \%$ & $11.27 \%$ & $20.25 \%$ & & 8 & 0 \\
\hline \multirow{2}{*}{$\mathrm{adv}$} & 3.1 .2 & $85.71 \%$ & $11.11 \%$ & $19.67 \%$ & \multirow{2}{*}{54} & 6 & 1 \\
\hline & 4.0 .0 & $75.00 \%$ & $11.11 \%$ & $19.35 \%$ & & 6 & 2 \\
\hline \multirow{2}{*}{$\mathrm{cmt}$} & 3.1 .2 & $100.00 \%$ & $9.68 \%$ & $17.65 \%$ & \multirow{2}{*}{31} & 3 & 0 \\
\hline & 4.0 .0 & $100.00 \%$ & $9.68 \%$ & $17.65 \%$ & & 3 & 0 \\
\hline \multirow{2}{*}{ sem } & 3.1 .2 & $66.67 \%$ & $9.52 \%$ & $16.67 \%$ & \multirow{2}{*}{21} & 2 & 1 \\
\hline & 4.0 .0 & $66.67 \%$ & $9.52 \%$ & $16.67 \%$ & & 2 & 1 \\
\hline \multirow{2}{*}{$\mathrm{cjc}$} & 3.1 .2 & $100.00 \%$ & $6.67 \%$ & $12.50 \%$ & \multirow{2}{*}{15} & 1 & 0 \\
\hline & 4.0 .0 & $100.00 \%$ & $6.67 \%$ & $12.50 \%$ & & 1 & 0 \\
\hline & 3.1 .2 & $100.00 \%$ & $2.78 \%$ & $5.41 \%$ & \multirow{2}{*}{72} & 2 & 0 \\
\hline & 4.0 .0 & $100.00 \%$ & $5.56 \%$ & $10.53 \%$ & & 4 & 0 \\
\hline \multirow{2}{*}{ reg } & 3.1 .2 & $80.00 \%$ & $3.17 \%$ & $6.11 \%$ & 126 & 4 & 1 \\
\hline & 4.0 .0 & $71.43 \%$ & $3.97 \%$ & $7.52 \%$ & 120 & 5 & 2 \\
\hline det & 3.1 .2 & $100.00 \%$ & $2.86 \%$ & $5.56 \%$ & 25 & 1 & 0 \\
\hline uet & 4.0.0 & $100.00 \%$ & $2.86 \%$ & $5.56 \%$ & 30 & 1 & 0 \\
\hline ley & 3.1 .2 & $100.00 \%$ & $1.67 \%$ & $3.28 \%$ & 60 & 1 & 0 \\
\hline $10 x$ & 4.0 .0 & $100.00 \%$ & $1.67 \%$ & $3.28 \%$ & 00 & 1 & 0 \\
\hline ali & 3.1 .2 & $0.00 \%$ & $0.00 \%$ & $0.00 \%$ & 2 & 0 & 0 \\
\hline & 4.0 .0 & $0.00 \%$ & $0.00 \%$ & $0.00 \%$ & & 0 & 2 \\
\hline orer & 3.1 .2 & $0.00 \%$ & $0.00 \%$ & $0.00 \%$ & 0 & 0 & 0 \\
\hline SCI & 4.0 .0 & $0.00 \%$ & $0.00 \%$ & $0.00 \%$ & 0 & 0 & 7 \\
\hline
\end{tabular}

Table 7.1: Effectiveness results for each error category of CoGrOO 3.1.2 and 4.0.0 according to Probi corpus. The category description can be found in Appendix B. 


\begin{tabular}{|c|c|c|c|c|c|c|}
\hline Category & Precision & Recall & $F_{1}$ & Target & TP & FP \\
\hline \hline ALL & $62.75 \%$ & $14.30 \%$ & $23.29 \%$ & 2616 & 374 & 222 \\
\hline con & $60.35 \%$ & $31.07 \%$ & $41.02 \%$ & 441 & 137 & 90 \\
\hline
\end{tabular}

Table 7.2: Effectiveness results for nominal agreement if the token a is ignored.

\subsection{Metro Corpus}

The grammar checker precision also increased in Metro Corpus, as shown in Table 7.3, increasing from $19.28 \%$ to $44.12 \%$ due to a decrease from 67 to 19 in the number of false positives, which is a significant improvement, but the number is still too high. Most of the false positives, 15, are related to nominal agreement.

Important changes were again found in con and cov categories, for which the number of false positives dramatically decreased. For nominal agreement, it decreased from 26 to 15 , and for verb agreement from 39 to 3 . No regressions were noticed in this experiment. The category follows the PROBI notation, and can be found in Appendix B.

\begin{tabular}{|c|c|c|c|c|c|c|c|}
\hline Category & Version & Precision & Recall & $F_{1}$ & Target & TP & FP \\
\hline \hline \multirow{2}{*}{ ALL } & 3.1 .2 & $19.28 \%$ & $30.19 \%$ & $23.53 \%$ & \multirow{2}{*}{53} & 16 & 67 \\
& 4.0 .0 & $44.12 \%$ & $28.30 \%$ & $34.48 \%$ & & 15 & 19 \\
\hline \multirow{2}{*}{ ren } & 3.1 .2 & $100.00 \%$ & $100.00 \%$ & $100.00 \%$ & \multirow{2}{*}{1} & 1 & 0 \\
& 4.0 .0 & $100.00 \%$ & $100.00 \%$ & $100.00 \%$ & & 1 & 0 \\
\hline \multirow{2}{*}{ cjc } & 3.1 .2 & $100.00 \%$ & $100.00 \%$ & $100.00 \%$ & \multirow{2}{*}{1} & 1 & 0 \\
& 4.0 .0 & $100.00 \%$ & $100.00 \%$ & $100.00 \%$ & & 1 & 0 \\
\hline \multirow{2}{*}{ cra } & 3.1 .2 & $71.43 \%$ & $62.50 \%$ & $66.67 \%$ & \multirow{2}{*}{8} & 5 & 2 \\
& 4.0 .0 & $83.33 \%$ & $62.50 \%$ & $71.43 \%$ & & 5 & 1 \\
\hline \multirow{2}{*}{ con } & 3.1 .2 & $18.75 \%$ & $75.00 \%$ & $30.00 \%$ & \multirow{2}{*}{8} & 6 & 26 \\
& 4.0 .0 & $25.00 \%$ & $62.50 \%$ & $35.71 \%$ & & 5 & 15 \\
\hline \multirow{2}{*}{ reg } & 3.1 .2 & $100.00 \%$ & $18.18 \%$ & $30.77 \%$ & \multirow{2}{*}{11} & 2 & 0 \\
& 4.0 .0 & $100.00 \%$ & $18.18 \%$ & $30.77 \%$ & & 2 & 0 \\
\hline \multirow{2}{*}{ ptn } & 3.1 .2 & $100.00 \%$ & $6.25 \%$ & $11.76 \%$ & \multirow{2}{*}{16} & 1 & 0 \\
& 4.0 .0 & $100.00 \%$ & $6.25 \%$ & $11.76 \%$ & & 1 & 0 \\
\hline \multirow{2}{*}{ ali } & 3.1 .2 & $0.00 \%$ & $0.00 \%$ & $0.00 \%$ & \multirow{2}{*}{1} & 0 & 0 \\
& 4.0 .0 & $0.00 \%$ & $0.00 \%$ & $0.00 \%$ & & 0 & 0 \\
\hline \multirow{2}{*}{ cov } & 3.1 .2 & $0.00 \%$ & $0.00 \%$ & $0.00 \%$ & \multirow{2}{*}{4} & 0 & 39 \\
& 4.0 .0 & $0.00 \%$ & $0.00 \%$ & $0.00 \%$ & & 0 & 3 \\
\hline \multirow{2}{*}{ det } & 3.1 .2 & $0.00 \%$ & $0.00 \%$ & $0.00 \%$ & \multirow{2}{*}{2} & 0 & 0 \\
& 4.0 .0 & $0.00 \%$ & $0.00 \%$ & $0.00 \%$ & & 0 & 0 \\
\hline \multirow{2}{*}{ res } & 3.1 .2 & $0.00 \%$ & $0.00 \%$ & $0.00 \%$ & \multirow{2}{*}{1} & 0 & 0 \\
& 4.0 .0 & $0.00 \%$ & $0.00 \%$ & $0.00 \%$ & & 0 & 0 \\
\hline
\end{tabular}

Table 7.3: Effectiveness results for each error category of CoGrOO 3.1.2 and 4.0.0 according to Metro corpus 


\subsection{Bosque}

The evaluation with Bosque corpus also demonstrated a significant improvement. As shown in Table 7.4, the total number of false positives dropped from 245 to 69, and the most important change is again in nominal and verb agreement categories. For the first, the number of false positive decreased from 134 to 38, and for the second from 94 to 17 . Most of the other categories kept the same number of false positives, except for the usage of an accent stress marker, which also improved, decreasing from 5 to 2 false positives. The Bosque corpus has 4,212 sentences.

\begin{tabular}{|c|c|c|}
\hline Category & Version & FP \\
\hline \hline \multirow{2}{*}{ ALL } & 3.1 .2 & 245 \\
& 4.0 .0 & 69 \\
\hline \multirow{2}{*}{ adv } & 3.1 .2 & 2 \\
& 4.0 .0 & 2 \\
\hline \multirow{2}{*}{ aha } & 3.1 .2 & 2 \\
& 4.0 .0 & 2 \\
\hline \multirow{2}{*}{ con } & 3.1 .2 & 134 \\
& 4.0 .0 & 38 \\
\hline \multirow{2}{*}{ cop } & 3.1 .2 & 1 \\
& 4.0 .0 & 1 \\
\hline \multirow{2}{*}{ cov } & 3.1 .2 & 94 \\
& 4.0 .0 & 17 \\
\hline \multirow{2}{*}{ cra } & 3.1 .2 & 5 \\
& 4.0 .0 & 2 \\
\hline \multirow{2}{*}{ ger } & 3.1 .2 & 6 \\
& 4.0 .0 & 6 \\
\hline \multirow{2}{*}{ ptn } & 3.1 .2 & 1 \\
& 4.0 .0 & 1 \\
\hline
\end{tabular}

Table 7.4: Effectiveness results for each error category of BASELINE according to Bosque corpus

\subsection{CoGrOO Community Corpus}

Both precision and recall improved in the CoGrOO Community Corpus, as shown in Table 7.5. The precision increased from $2.47 \%$ to $28.24 \%$ due to a reduction from 158 to 61 in the number of false positives. Again, most of the false positives, 37, are related to noun agreement. Recall improved from $3.85 \%$ to $23.08 \%$, the number of true positives increased from 4 to 24 , most related to verb agreement. 


\begin{tabular}{|c|c|c|c|c|c|c|c|}
\hline Category & Version & Precision & Recall & $F_{1}$ & Target & $\mathrm{TP}$ & $\mathrm{FP}$ \\
\hline \multirow{2}{*}{ ALL } & 3.1 .2 & $2.47 \%$ & $3.85 \%$ & $3.01 \%$ & \multirow{2}{*}{104} & 4 & 158 \\
\hline & 4.0 .0 & $44.12 \%$ & $28.30 \%$ & $34.48 \%$ & & 24 & 61 \\
\hline \multirow{2}{*}{$\mathrm{cov}$} & 3.1 .2 & $0.00 \%$ & $0.00 \%$ & $0.00 \%$ & \multirow{2}{*}{40} & 0 & 1 \\
\hline & 4.0.0 & $93.75 \%$ & $37.50 \%$ & $53.57 \%$ & & 15 & 1 \\
\hline \multirow{2}{*}{ con } & 3.1 .2 & $2.08 \%$ & $12.00 \%$ & $3.55 \%$ & \multirow{2}{*}{25} & 3 & 141 \\
\hline & 4.0 .0 & $17.78 \%$ & $32.00 \%$ & $22.86 \%$ & & 8 & 37 \\
\hline \multirow{2}{*}{ cra } & 3.1 .2 & $25.00 \%$ & $9.09 \%$ & $13.33 \%$ & \multirow{2}{*}{11} & 1 & 3 \\
\hline & 4.0 .0 & $33.33 \%$ & $9.09 \%$ & $14.29 \%$ & & 1 & 2 \\
\hline \multirow{2}{*}{$\mathrm{adv}$} & 3.1 .2 & $0.00 \%$ & $0.00 \%$ & $0.00 \%$ & \multirow{2}{*}{1} & 0 & 2 \\
\hline & 4.0.0 & $0.00 \%$ & $0.00 \%$ & $0.00 \%$ & & 0 & 3 \\
\hline \multirow{2}{*}{ aha } & 3.1 .2 & $0.00 \%$ & $0.00 \%$ & $0.00 \%$ & \multirow{2}{*}{3} & 0 & 5 \\
\hline & 4.0 .0 & $0.00 \%$ & $0.00 \%$ & $0.00 \%$ & & 0 & 5 \\
\hline \multirow{2}{*}{ ali } & 3.1 .2 & $0.00 \%$ & $0.00 \%$ & $0.00 \%$ & \multirow{2}{*}{1} & 0 & 0 \\
\hline & 4.0 .0 & $0.00 \%$ & $0.00 \%$ & $0.00 \%$ & & 0 & 0 \\
\hline \multirow{2}{*}{ cop } & 3.1 .2 & $0.00 \%$ & $0.00 \%$ & $0.00 \%$ & \multirow{2}{*}{5} & 0 & 0 \\
\hline & 4.0 .0 & $0.00 \%$ & $0.00 \%$ & $0.00 \%$ & & 0 & 0 \\
\hline \multirow{2}{*}{ ger } & 3.1 .2 & $0.00 \%$ & $0.00 \%$ & $0.00 \%$ & \multirow{2}{*}{0} & 0 & 4 \\
\hline & 4.0.0 & $0.00 \%$ & $0.00 \%$ & $0.00 \%$ & & 0 & 4 \\
\hline \multirow{2}{*}{ lex } & 3.1 .2 & $0.00 \%$ & $0.00 \%$ & $0.00 \%$ & \multirow{2}{*}{1} & 0 & 0 \\
\hline & 4.0 .0 & $0.00 \%$ & $0.00 \%$ & $0.00 \%$ & & 0 & 0 \\
\hline \multirow{2}{*}{ ort } & 3.1 .2 & $0.00 \%$ & $0.00 \%$ & $0.00 \%$ & \multirow{2}{*}{4} & 0 & 0 \\
\hline & 4.0 .0 & $0.00 \%$ & $0.00 \%$ & $0.00 \%$ & & 0 & 0 \\
\hline \multirow{2}{*}{ ptn } & 3.1 .2 & $0.00 \%$ & $0.00 \%$ & $0.00 \%$ & \multirow{2}{*}{2} & 0 & 0 \\
\hline & 4.0 .0 & $0.00 \%$ & $0.00 \%$ & $0.00 \%$ & & 0 & 0 \\
\hline \multirow{2}{*}{ reg } & 3.1 .2 & $0.00 \%$ & $0.00 \%$ & $0.00 \%$ & \multirow{2}{*}{6} & 0 & 1 \\
\hline & 4.0 .0 & $0.00 \%$ & $0.00 \%$ & $0.00 \%$ & & 0 & 1 \\
\hline \multirow{2}{*}{ ren } & 3.1 .2 & $0.00 \%$ & $0.00 \%$ & $0.00 \%$ & \multirow{2}{*}{0} & 0 & 1 \\
\hline & 4.0 .0 & $0.00 \%$ & $0.00 \%$ & $0.00 \%$ & & 0 & 1 \\
\hline \multirow{2}{*}{ sem } & 3.1 .2 & $0.00 \%$ & $0.00 \%$ & $0.00 \%$ & ? & 0 & 0 \\
\hline & 4.0 .0 & $0.00 \%$ & $0.00 \%$ & $0.00 \%$ & 2 & 0 & 0 \\
\hline Ve & 3.1 .2 & $0.00 \%$ & $0.00 \%$ & $0.00 \%$ & 3 & 0 & 0 \\
\hline Ver & 4.0 .0 & $0.00 \%$ & $0.00 \%$ & $0.00 \%$ & 3 & 0 & 0 \\
\hline
\end{tabular}

Table 7.5: Effectiveness results for each error category of CoGrOO 3.1.2 and 4.0.0 according to Community corpus

\subsection{Comparison to Other Systems}

This section compares the effectiveness in terms of precision and recall of CoGrOO and other available systems. Microsoft Word 2010 was used to evaluate ReGra, and LibreOffice Writer 3.6.3.2 to evaluate Vero Grammar Checker 3.0.0. The tests were performed by manually copying the corpus sentences to the text editors. Table 7.6 summarizes the results for different corpora.

In all experiments, spelling mistakes were not counted, nor were mechanical errors like extra spaces between words and the final punctuation mark. The last was not considered 


\begin{tabular}{|c|c|c|c|c|c|c|c|}
\hline & Grammar Checker & Target & $\mathrm{TP}$ & $\mathrm{FP}$ & Precision & Recall & $F$ \\
\hline \multirow{3}{*}{ 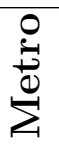 } & ReGra & \multirow{3}{*}{55} & 26 & 47 & $35.62 \%$ & $47.27 \%$ & $40.63 \%$ \\
\hline & Vero & & 12 & 6 & $66.67 \%$ & $21.82 \%$ & $32.88 \%$ \\
\hline & $\mathrm{CoGrOO}$ & & 15 & 19 & $44.12 \%$ & $27.27 \%$ & $33.71 \%$ \\
\hline \multirow{3}{*}{ ঠٌ } & ReGra & \multirow{3}{*}{104} & 49 & 32 & $60.49 \%$ & $47.12 \%$ & $52.97 \%$ \\
\hline & Vero & & 36 & 8 & $81.82 \%$ & $34.62 \%$ & $48.65 \%$ \\
\hline & $\mathrm{CoGrOO}$ & & 24 & 61 & $28.24 \%$ & $23.08 \%$ & $25.40 \%$ \\
\hline \multirow{3}{*}{$\begin{array}{l}\text { D } \\
0 \\
0 \\
n\end{array}$} & ReGra & & & 606 & & & \\
\hline & Vero & & & 52 & & & \\
\hline & $\mathrm{CoGrOO}$ & & & 69 & & & \\
\hline
\end{tabular}

Table 7.6: Comparison between CoGrOO and other Brazilian Portuguese grammar checkers in terms of precision, recall and $F_{1}$. Evaluations were performed in Metro, Community and Bosque corpora.

firstly because the corpus had many of these errors due to the markup, and secondly because the analyzed version of CoGrOO does not implement such rules, and considering it would cause imbalanced results.

As expected, the commercial system Microsoft Word 2010 with ReGra grammar checker was more effective in experiments, at least in terms of recall. As CoGrOO, this grammar checker also applies NLP to analyze the text, leading to a high coverage, but also to a high number of false positives. This was evident while evaluating Bosque corpus, which has four thousand sentences. The number of false positives exceeded six hundred.

ReGra was also effective in Community and Metro corpus, scoring the highest number of true positives, and a reasonable number of false positives.

Not surprisingly, Vero Grammar Checker was also highly effective, especially in terms of precision, scoring better than both ReGra and CoGrOO in Metro and Community corpus. The low number of false positives of this system is due to the fact that it does not perform any NLP classification that would cause ambiguity, like part-of-speech tagging. On the one hand it is good for the precision, but for the coverage it is not. Most of its rules are trivial, but since they are selected from the most common mistakes for Brazilian Portuguese writers, they are very effective.

The lesson learned from this comparison is that often it is better to have effective simple rules instead of complex and error prone deep NLP analysis. Also, CoGrOO would benefit from expanding its rules taking advantage of the NLP analysis it can do efficiently, and in this case it would perform better than the Vero Grammar Checker. 


\section{Chapter 8}

\section{Conclusions}

This work is broad in the sense that it covers many of the basic Natural Language Processing tasks, but it is also shallow because it does not deepen any of these tasks, even though frequently the effectiveness of the modules achieved values close to the state-of-theart. The major purpose of this work was to improve the Portuguese grammar checker by tuning its language processing modules, which as shown in the experimental section, was very effective.

There is two important lessons learned from this work. Firstly, the ambiguity problem which haunts any natural language related work. Often, to perform a task as simple as sentence tokenization, it is necessary to have semantic knowledge and make context based inferences that is simply too difficult to reproduce in shallow language processing. Secondly, the task of grammar checking is especially challenging due to the fact that sentences containing errors are even more difficult to discern, because they do not follow the conventional grammar structure, and they are constructed in an unpredictable manner.

\subsection{Major Contributions}

The main contributions of this dissertation are: a) a more effective Libre|OpenOffice grammar checker for Portuguese; b) the identification of key issues in training Apache OpenNLP models for Portuguese; $c$ ) efficient NLP modules for Portuguese Natural Language Processing; $d$ ) contributions to Open Source projects, like CoGrOO and Apache OpenNLP; and e) contributions to other academic projects. The following sections detail each contribution.

\subsubsection{A More Effective Libre|OpenOffice Grammar Checker}

The CoGrOO version proposed by this work is significantly more effective, especially in terms of precision. For PROBI corpus the precision raised from $23.21 \%$ to $60.03 \%$, and for Metro corpus from $19.28 \%$ to $44.12 \%$. For Bosque corpus, which have been proof-read, the number of false positives decreased from 245 to 69 in 4,212 sentences. This improvement 
is significant to the user experience because the less false positives, the less the user is interrupted by the grammar checker and the higher is the user's trust in it.

Recall values did not improve significantly. To improve recall it would be required adding new error rules, which was left for future studies.

\subsubsection{Identification of Key Issues in Training Apache OpenNLP Models for Portuguese}

Most of the issues training the Apache OpenNLP Portuguese models are related to the training corpora, which are Floresta Virgem and Bosque. Other issues are intrinsic to Portuguese processing and were also pointed out by Silva (2007), which were also confirmed in this work.

- Sentence boundary detector

- Ambiguity and ambivalence of the period (full stop) symbol (Silva, 2007);

- Abbreviations;

- Quotations, parentheses, etc, which in the training corpus (VCP) can split sentences according to semantic information (Afonso et al., 2001).

- Tokenizer

- Token-ambiguous strings (Silva, 2007);

- Hyphenated words, because the training corpus uses a deeper contextual analysis, which is not available at this level of shallow processing;

- Tokenization of proper names, which are grouped as one token in the training corpus and requires some preprocessing.

- Name Finder

- Divergences in what is a proper name between Virgem CETENFolha and Bosque prevents using one or another: it would affect the POS Tagger;

- Training with larger corpus either takes too long or generates unfeasibly large models;

- Grouped names can hide grammar errors (Palácio do Alvorada).

- Contractions Finder

- It is not a traditional task, but is required to properly use the POS Tagger;

- Based on Name Finder, but much more effective after some customizations. 
- POS Tagger

- It is too dependent on the previous modules: its effectiveness depends on Sentence Detector, Tokenizer, Name Finder and Contractions fine-tuned to output data as close as possible as is expected for the POS tagger input;

- Multi-word expressions in the training data; it is not clear which is the best approach to handle it;

- Its effectiveness depends too much on the POS Dictionary, whose entries classification should not diverge from the training corpus;

- The customization of the module improved the results, especially for difficult cases like a and que.

- Featurizer

- Using surrounding context helps, but it can also lead to misclassifications if the sentence has agreement errors;

- The feature dictionary degraded the accuracy, mainly because it sometimes diverges from the corpus, but the dictionary is used because it makes the output more reliable when there is agreement errors.

- Chunker

- It depends on a heuristic to translate the tree format to chunks, and the implementation of this heuristic might have errors.

- Shallow Parsing

- It also depends on a heuristic to translate the tree format to syntactic chunks, and the implementation of this heuristic might have errors;

- The results are not good in sentences with multiple clauses.

\subsubsection{Efficient Models for Portuguese Natural Language Processing}

This work also produced efficient models for Portuguese NLP, which can be used by any other system. The models can be loaded directly from the latest Apache OpenNLP (version 1.5.3 or better) API, which includes a new feature that allows loading customized context generators, sequence validators and resources, or can also be used from the CoGrOO API.

Table 8.1 details the effectiveness of the models, and Table 8.2 details how the model was trained. 


\begin{tabular}{|c||c|c|c|c|}
\hline Module & Accuracy & Precision & Recall & $F_{1}$ \\
\hline \hline Sentence Detector & & 99.215 & 99.259 & 99.237 \\
\hline Tokenizer & & 99.949 & 99.933 & 99.941 \\
\hline Name Finder & & 89.019 & 86.002 & 87.484 \\
\hline Contraction Finder & & 99.948 & 99.945 & 99.947 \\
\hline POS Tagger & 96.291 & & & \\
\hline Featurizer & 96.743 & & & \\
\hline Chunker & & 96.341 & 96.431 & 96.386 \\
\hline Chunk Head Finder & 99.777 & & & \\
\hline Shallow Parser & & 83.818 & 82.493 & 83.150 \\
\hline
\end{tabular}

Table 8.1: Final 10-fold cross-validation score for each of the CoGrOO models.

\begin{tabular}{|c||c|c|c|c|}
\hline Module & Algorithm & Cutoff & Corpus & Options \\
\hline \hline Sentence Detector & Perceptron & 0 & VCF & ACP, ABB \\
\hline Tokenizer & Perceptron & 0 & VCF & ABB, AO \\
\hline Name Finder & Perceptron & 0 & CF & \\
\hline Contraction Finder & Perceptron & 8 & VCF & \\
\hline POS Tagger & Perceptron & 0 & CF & DIC, MDIC, CCG, AC \\
\hline Featurizer & Perceptron & 0 & CF & DIC, WHNCS \\
\hline Chunker & Perceptron & 0 & CF & \\
\hline Chunk Head Finder & Maxent & 8 & CF & \\
\hline Shallow Parser & Perceptron & 0 & CF & \\
\hline
\end{tabular}

Table 8.2: The configurations which generated the best models. VCF stands from Virgem CETENFolha, and CF stands for Bosque CETENFolha. Details about the meaning of the options can be found in the experimental section.

\subsubsection{Contributions to Open Source}

This work directly contributed to some Open Source projects. The main contribution is to the open source Portuguese grammar checker CoGrOO. The grammar checker developed here is called CoGrOO 4.0.0, and both source code and distributable are available on the official website ${ }^{1}$.

The second most important contribution is to the project Apache OpenNLP. This work contributed several improvements to the project code base, including:

- Formatters to read the Arvores Deitadas corpus;

- Extension capabilities which allows easier customizations of context generators, sequence validators and resources;

- The ability to plug in evaluation listeners;

- A listener that outputs details for each error;

\footnotetext{
${ }^{1}$ CoGrOO official website, http://cogroo.org, last accessed on 12/10/2012
} 
- A fine grained evaluation report for POS Tagger (tokens with the highest number of errors, most frequent tokens, detailed accuracy by pos tag, general confusion matrix and confusion matrix for each token);

- A detailed F-Measure report that can be used by every tool that outputs $F_{1}$ (detailed global score, detailed score for each output type, target, true positives and false positives count);

and many others. A complete list can be found at the Apache OpenNLP issue tracker ${ }^{2}$. Another major contribution is the Brazilian Portuguese dictionaries for the morphological analyzer and spell checker Jspell. The translation of the dictionary is a work in progress, performed by the contributors of the CoGrOO project. The source code is available at GitHub ${ }^{3}$.

During the development of this work the CoGrOO analyzers were also wrapped as Apache UIMA annotators. The annotators and their source code are available online, as described in the related paper (Silva et al., 2010).

Finally, but no less important, during this work, contributions were made to the development of the CoGrOO Comunidade portal, described in Section 3.4.

\subsubsection{Contributions to Other Research Projects}

CoGrOO and its internal modules have been used by a number of research projects. Since it is a freely available open source project, only users who had contacted the development team at least once are known. The following list demonstrates the range of research projects using it.

OnAIR (Ontology Aided Information Retrieval) is a system developed for searching within digital video databases. One of its researchers (Torres, 2012) used the internal modules of the grammar checker to analyze audio transcription linguistic information. He studied how to use linguistic information to learn ontologies from text and used the CoGrOO POS Tagger and Chunker as part of the computational tools. Another researcher (Luz, 2012) used pos tagging, chunking and shallow parser to analyze natural language queries. A researcher from the same group, Marta R. Costa-jussà, measured substantial improvement in statistical bilingual translation by embedding morphological annotations to the data. For this work, which has not yet been published, she used CoGrOO to analyze Portuguese.

CoGrOO annotators have been used also in health informatics. Oleynik et al. (2010) developed a study on how CoGrOO POS Tagger performs in clinical data, and recently he has been applying CoGrOO annotators to the task of Information Retrieval on Clinical Data (Oleynik, 2012). It was also used by Souza (2012) in her studies of pattern identification in

\footnotetext{
${ }^{2}$ Apache OpenNLP issue tracker, https://issues.apache.org/jira/secure/IssueNavigator.jspa?reset= true\&jqlQuery $=$ project $+\% 3 \mathrm{D}+\mathrm{OPENNLP}+\mathrm{AND}+$ assignee $+\% 3 \mathrm{D}+$ colen

${ }^{3}$ JSpell.br website, http://github.com/cogroo/jspell.br
} 
discharge summaries, and by Oliveira et al. (2012) in a study to classify whether there is continuity of care in hospital discharge summaries.

The annotators have also been used in linguistic research. This is the case of Matte et al. (2012), who annotated texts with morphology and syntactic information, and later allow researchers to use these annotation in semiotic research.

Finally, two engineering projects. PoliLIBRAS ${ }^{4}$ is a translator from Portuguese and LIBRAS, the Brazilian Sign Language, which uses both morphologic and syntactic information in the translation process. Another interesting initiative is the discourse management software of the social robot proposed by Alfenas and Pereira-Barretto (2012), which uses the syntactic analysis provided by CoGrOO to allow for a natural conversation between humans and robots.

\subsection{Future Studies}

This work presented quite a few of possibilities for future studies. Many of them are directly related to the grammar checker itself, and others related to the development of its internal modules.

Improving the grammar checker precision was the main objective of this work. The small improvement in recall is due to the more effective NLP components, but a significant improvement in recall would be possible only with additional rules and checkers, which should enlarge grammar error coverage. As indicated by the experimental phase, many rules present counterexamples which should be revised to reduce false positives. Adding a machine learn component trained to identify grammar errors is a challenge that would also improve coverage.

Many NLP modules should benefit from a deeper effort in its development, which was not the focus of this work. For example, a deeper effort should allow for improved handling of the problem of preposition a versus article a. Exploring other approaches to handle multi-word expressions and contractions should also improve the grammar checker.

Adding new modules to the pipeline should also help; for example, a clause identifier could improve the shallow parser accuracy for sentences with multiple clauses. Yet in shallow parsing, design a better method to choose the number and gender of a subject is required.

\footnotetext{
${ }^{4}$ PoliLIBRAS official website, http://www.polilibras.com.br, last accessed on 12/17/2012
} 


\section{Appendix A}

\section{Base POS Tagset}

This appendix describes the tagset used by different modules of CoGrOO.

\section{A.1 Class tags}

The POS tagset used in CoGrOO 1.0 to 3.1 is based on the Constraint Grammar category set of Palavras ${ }^{1}$, and the one used by the POS tagger introduced in this work is based on the Floresta tagset ${ }^{2}$.

The subset of the tags used by CoGrOO are included in Table A.1 for reference.

\section{A.2 Inflection Tags}

The inflection tags used by all CoGrOO versions are based on the Constraint Grammar category set of Palavras. Those actually used by the grammar checker are listed in Table A.2.

\section{A.3 Chunk tags}

The chunk tagset used by CoGrOO is also based on the one proposed by the Floresta project. Until CoGrOO 3.1.2, only noun and verb phrase tags were used. This work introduced a few more tags, as shown in Table A.3.

\section{A.4 Shallow Parser tags}

The shallow parser tagset used by CoGrOO is also based on the one proposed by Floresta project, this time derived from the clause level tagset. Until CoGrOO 3.1.2 only subject and

\footnotetext{
${ }^{1}$ The Constraint Grammar category set of Palavrashttp://beta.visl.sdu.dk/visl/pt/info/portsymbol.html, last accessed on $12 / 13 / 2012$

${ }^{2}$ Grammatical categories used in the Floresta projecthttp://beta.visl.sdu.dk/visl/pt/symbolset-floresta. html, last accessed on 12/13/2012
} 


\begin{tabular}{|l|l|l|}
\hline Tag CG & Tag Palavras & Description \\
\hline N & n & Nouns \\
PROP & prop & Proper nouns (names) \\
SPEC & - & Specifiers \\
DET & - & Determiners \\
- & art & Article \\
PERS & pron-pers & Personal pronouns \\
- & pron-det & Determinative pronouns \\
- & pron-indp & Independent pronouns \\
ADJ & adj & Adjectives \\
- & n-adj & Noun or adjective \\
ADV & adv & Adverbs \\
V & - & Verbs \\
- & v-fin & Finite verbs \\
- & v-inf & Infinitive verbs \\
- & v-pcp & Participle verbs \\
- & v-ger & Gerund verbs \\
NUM & num & Numerals (cardinals) \\
PRP & prp & Preposition \\
KS & conj-s & Subordinating conjunctions \\
KC & conj-c & Coordinating conjunctions \\
IN & intj & Interjections \\
EC & - & Hyphen-separated prefix \\
\hline
\end{tabular}

Table A.1: Subset of the POS class tagset used by CoGrOO. The first column is based on the Constraint Grammar category set of Palavras and is used by CoGrOO 1.0 to 3.1.2. The second column is based on the Floresta tagset and is used by the modules developed in this work.

verb tags were used. This work introduced a few more tags as outcome. Table A.4 details the tagset. 


\begin{tabular}{lll}
\hline Group & Tag & Inflection \\
\hline \multirow{2}{*}{ Gender } & M & masculine \\
& F & feminine \\
\hline \multirow{2}{*}{ Number } & S & singular \\
& P & plural \\
\hline \multirow{4}{*}{ Case } & NOM & nominative \\
& ACC & accusative \\
& DAT & dative \\
& PIV & prepositive \\
\hline \multirow{4}{*}{ Person } & 1 & first person \\
& 2 & second person \\
& 3 & third person \\
\hline \multirow{5}{*}{ Tense } & PR & present tense \\
& IMPF & imperfect \\
& PS & simple perfect \\
& MQP & pluperfect \\
& FUT & future \\
& COND & conditional \\
\hline \multirow{3}{*}{ Mood } & IND & indicative \\
& SUBJ & subjunctive \\
& IMP & imperative \\
\hline \multirow{5}{*}{ Finiteness } & VFIN & finite verb \\
& PCP & infinitive \\
& GER & participle \\
& gerund \\
\hline & &
\end{tabular}

Table A.2: Subset of the inflection tagset from the Constraint Grammar category set of Palavras.

\begin{tabular}{|l|l|}
\hline Tag & Description \\
\hline NP & Noun phrase \\
VP & Verbal phrase \\
PP & Prepositional phrase \\
ADVP & Adverbial phrase \\
\hline
\end{tabular}

Table A.3: Subset of the group tagset used by CoGrOO based on the Floresta tagset. 


\begin{tabular}{|l|l|}
\hline Tag & Description \\
\hline ACC & Direct (accusative) object \\
ADVL & Adjunct adverbial \\
APP & Adnominal apposition \\
DAT & Dative object \\
OC & Object complement \\
P & Predicator \\
PIV & Prepositional object \\
SA & Adverb complement \\
SC & Subject complement \\
SUBJ & Subject \\
\hline
\end{tabular}

Table A.4: Subset of the clause level tagset from Floresta used by CoGrOO based. 


\section{Appendix B}

\section{PROBI Error Categories}

Table B describes each of the grammar errors from PROBI corpus. Table B.2 associates each category with the CoGrOO's rules. CoGrOO does not cover the following error categories: abr, ace, adj, arc, bde, cap, cli, det, esp, est, lex, mec, mor, neo, nol, num, ond, ort, par, ple, pre, prq, ptp, rep and res.

\begin{tabular}{|c|c|c|}
\hline Category & Description & Details \\
\hline $\operatorname{abr}(14)$ & Use of acronyms & Indicates misuse of acronyms and abbreviations \\
\hline ace $(65)$ & Use of accent & $\begin{array}{l}\text { Indicates a problem in the use of accent mark } \\
\text { (single or displaced accents, excess or lack of } \\
\text { accents) }\end{array}$ \\
\hline $\operatorname{adj}(23)$ & Use of adjectives & Indicates a problem in the use of adjectives \\
\hline $\operatorname{adv}(54)$ & Use of adverbs & Indicates a problem in the use of adverbs \\
\hline aha $(29)$ & Use of há/a & Indicates change from há by a or vice versa \\
\hline ali $(2)$ & Others & Problems that do not fit in any other category \\
\hline $\operatorname{arc}(2)$ & Use of archaisms & $\begin{array}{l}\text { Indicates the use of archaic expressions or that } \\
\text { are in disuse in Portuguese }\end{array}$ \\
\hline bde $(0)$ & Balance of delimiters & $\begin{array}{l}\text { Indicates delimiters (parentheses, bracket, } \\
\text { quotes) unbalanced }\end{array}$ \\
\hline $\operatorname{cap}(4)$ & Capitalization & Indicates improper use of uppercase \\
\hline $\operatorname{cjc}(15)$ & Use of conjunctions & $\begin{array}{l}\text { Indicates problems in the use of conjunctions } \\
\text { and conjunctive phrases }\end{array}$ \\
\hline $\operatorname{cli}(9)$ & Use of cliché & Indicates use of fixed expressions \\
\hline $\operatorname{cmt}(31)$ & $\begin{array}{l}\text { Agreement between ver- } \\
\text { bal modes and tenses }\end{array}$ & $\begin{array}{l}\text { Indicates a lack of agreement between the forms } \\
\text { of the verbs }\end{array}$ \\
\hline $\operatorname{con}(441)$ & Nominal agreement & $\begin{array}{l}\text { Indicates a lack of agreement between the nom- } \\
\text { inal head and their nominal adjunct or predica- } \\
\text { tive }\end{array}$ \\
\hline cop $(140)$ & Pronoun placement & $\begin{array}{l}\text { Indicates problems in the use of proclisis, en- } \\
\text { clitic or mesoclisis }\end{array}$ \\
\hline
\end{tabular}


cov (562) Verbal agreement

cra (385) Use of crase

$\operatorname{det}(35) \quad$ Use of articles and determiners

esp (1) Use of space

est (1) Use of foreign words

ger (0) Use of gerund

lex (60) Lexical inadequacy

mal (10) Use of mau/mal

mec (5) Mechanical problems

mor (77) Morphology problems

neo (0) Use of neologisms

nol (52) Use of lexical notations

num (0) Use and spelling of numerals

ond (15) Use of onde/aonde

ort (157) Spelling

par (19) Use of paronymous

ple (0) Use of vulgarism

pre (67) Use of prepositions

pro (71) Use of pronouns

prq (1) Use of por que

ptn (32) Use of punctuation

ptp (9) Use of participle

reg (126) Verbal regency

ren (1) Nominal regency
Indicates a lack of agreement between subject and verb

Indicates a problem using the crase accent $(\mathrm{a}+$ a)

Indicates problems in the use of articles and determiners

Indicates problems in the use of blanks (excess of)

Indicates use of foreign words (estrangeirismo)

Indicates improper use of the gerund (gerundismo)

Indicates misuse of words (the words do not fit the context) or fixed expressions

Indicates the exchange of mau for mal or vice versa

Indicates typing problems

Indicates problems in the process of structuring and formation of words

Indicates the use of neologisms

Indicates misuse of the hyphen, quotation marks and other lexical notations

Indicates problem in the use or spelling of numerals

Indicates the exchange of onde for aonde or vice versa

Indicates spelling errors

Indicates the misuse of paronymous words (parônimo)

Indicates use of vulgarism plebeísmo

Indicates the misuse of prepositions and prepositional phrases

Indicates the misuse of pronouns

Indicates the exchange of por que, por quê, porque e por quê

Indicates the misuse of punctuation marks Indicates improper use of the past participle Indicates problems with regency of verbs Indicates problems with the regency of nouns, adjectives and adverbs 
rep (6) Excessive repetition of Indicates the absence of lexical substitution words mechanisms (lexical cohesion problem)

res (0) Excessive repetition of Repetition of words and symbols (mechanical symbols error)

sem (21) Severe pleonasm Indicates a severe pleonasm

ver (72) Use of verbs Indicates misuse of verbal forms

Table B.1: The error categories of PROBI corpus. The numbers in parenthesis indicates the occurrences of each error in the corpus. The total number of errors is 2616, and the total number of sentences 11625 (Martins, 2002).

\begin{tabular}{ll}
\hline PROBI Category & CoGrOO Rule \\
\hline adv & $38 ; 39$ \\
adv|con & 92 \\
aha & $46 ; 47$ \\
ali & $25 ; 26 ; 27 ; 59$ \\
cjc & 106 \\
cmt & $76 ; 77$ \\
con & $17 ; 18 ; 19 ; 20 ; 21 ; 22 ; 23 ; 24 ; 28 ; 29 ; 30 ;$ \\
& $31 ; 32 ; 33 ; 34 ; 35 ; 36 ; 37 ; 40 ; 41 ; 83 ; 95 ;$ \\
& $103 ; 104 ; 105 ; 114 ; 115 ; 124$ \\
cop & $61 ; 62 ; 63 ; 64 ; 65 ; 66 ; 67 ; 68 ; 69 ; 70 ; 71 ;$ \\
& $72 ; 73 ; 74$ \\
cop|pro & 52 \\
cov & $42 ; 44 ; 45 ; 48 ; 49 ; 50 ; 51 ; 60 ; 117 ; 118 ;$ \\
& $119 ; 120$ \\
cov|reg & 43 \\
cra & $1 ; 2 ; 3 ; 4 ; 5 ; 6 ; 7 ; 8 ; 9 ; 10 ; 11 ; 12 ; 13 ;$ \\
& $14 ; 15 ; 16 ; 78 ; 79 ; 80 ; 81 ; 82 ; 84 ; 85 ; 87 ;$ \\
ger & $89 ; 91 ; 93 ; 94 ; 108$ \\
mal & 121 \\
pro & $57 ; 58$ \\
ptn & $53 ; 54 ; 55 ; 56$ \\
reg & $111 ; 112 ; 113$ \\
ren & $86 ; 90 ; 96 ; 97 ; 98 ; 99 ; 100 ; 101 ; 102 ; 107 ;$ \\
sem & 109 \\
ver & $88 ; 110$ \\
& $122 ; 123$ \\
& 75
\end{tabular}

Table B.2: Association between CoGrOO's rules and the PROBI categories. 


\section{Appendix C}

\section{Source Code, Libraries and Tools}

This chapter offers an overview of the source code and instructions on how to obtain it. The objective is not to explain the source in detail, but to provide hints on how to start working with the code, try the grammar checker or the NLP library and to reproduce the experiments carried out during the development of this work.

\section{C.1 License}

CoGrOO source code is distributed under Apache License 2.0 ${ }^{1}$. The project dependencies and their licenses are in Table C.1.

\begin{tabular}{|c|c|c|c|}
\hline Library & Indirect & Version & License \\
\hline Apache Commons Codec & yes & 1.4 & Apache 2.0 \\
Apache Commons CLI & yes & 1.2 & Apache 2.0 \\
Apache Commons Lang & yes & 2.6 & Apache 2.0 \\
Apache Log4j & & 1.2 .17 & Apache 2.0 \\
Apache OpenNLP Maxent & yes & 3.0 .3 & Apache 2.0 \\
Apache OpenNLP Tools & & 1.5 .3 & Apache 2.0 \\
Apache OpenOffice Libraries & & 3.0 .1 & Apache 2.0 \\
Guava & & 11.0 .2 & Apache 2.0 \\
HPPC Collections & yes & 0.4 .1 & Apache 2.0 \\
JAXB (JSR 222) API & & 2.2 .5 & CDDL 1.1 \\
JSR305 & yes & 1.3 .9 & Apache 2.0 \\
JWNL & yes & 1.3 .3 & BSD \\
Morfologik & & 1.5 .3 & BSD \\
\hline
\end{tabular}

Table C.1: Dependencies of CoGrOO. A library is an indirect dependency if it is a dependency of a 3rd party library.

\footnotetext{
${ }^{1}$ Apache License 2.0, http://www.apache.org/licenses/LICENSE-2.0, last accessed on 12/28/2012
} 


\section{C.2 Download the Source}

Figure C.1 shows some basic statistics of the source code actually used by the grammar checker, which excludes projects related to grammar checker evaluation, which are under the cogroo-eval directory. The code has almost 22 thousand lines of code and a total of 308 classes.

\section{Lines of code}

21.790

36.307 lines

10.345 statements

256 files

\section{Classes}

308

55 packages

1.666 methods

164 accessors

Figure C.1: Statistics of the grammar checker source code.

The source code is hosted at GitHub, under the project cogroo/cogroo4. The complete project URL is http://github.com/cogroo/cogroo4, last accessed on 12/28/2012. The label v4.0.0-wc_tesis enables downloading the source code as it was by the time this document was written. The following listing shows how to obtain it using Git. Alternatively, it is possible to download it as a zip archive from the URL https://github.com/cogroo/cogroo4/ archive/v4.0.0-wc_tesis.zip, last accessed on 12/28/2012.

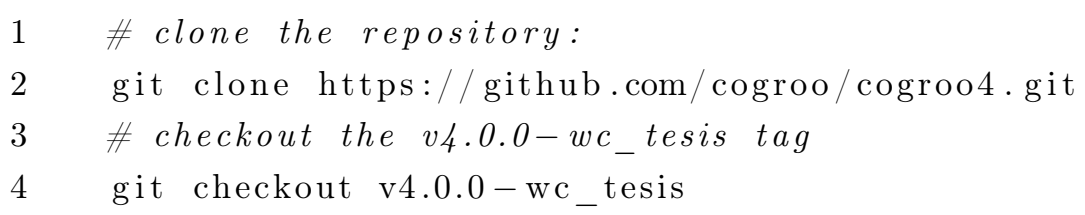

Code 2: Clone and checkout the grammar checker version produced by this work.

The command sequence will create a directory structure similar to the one in Table C. Each directory contains one or more Maven projects ${ }^{2}$, which are described below:

cogroo/ The main project aggregator.

cogroo-eval/ Contains several projects used to evaluate the NLP modules and grammar checker.

cogroo-dict Converts Jspell.br dictionaries to a format that can be used by CoGrOO.

cogroo-nlp Contains the basic classes to use CoGrOO models from OpenNLP. For example, customized context generators and validators, customized resources loaders, like POS dictionaries and others.

cogroo-ann Implementations of Analyzers, Pipelines, Document, Sentence etc.

\footnotetext{
${ }^{2}$ Apache Maven build manager, http://maven.apache.org, last accessed on 12/28/2012
} 


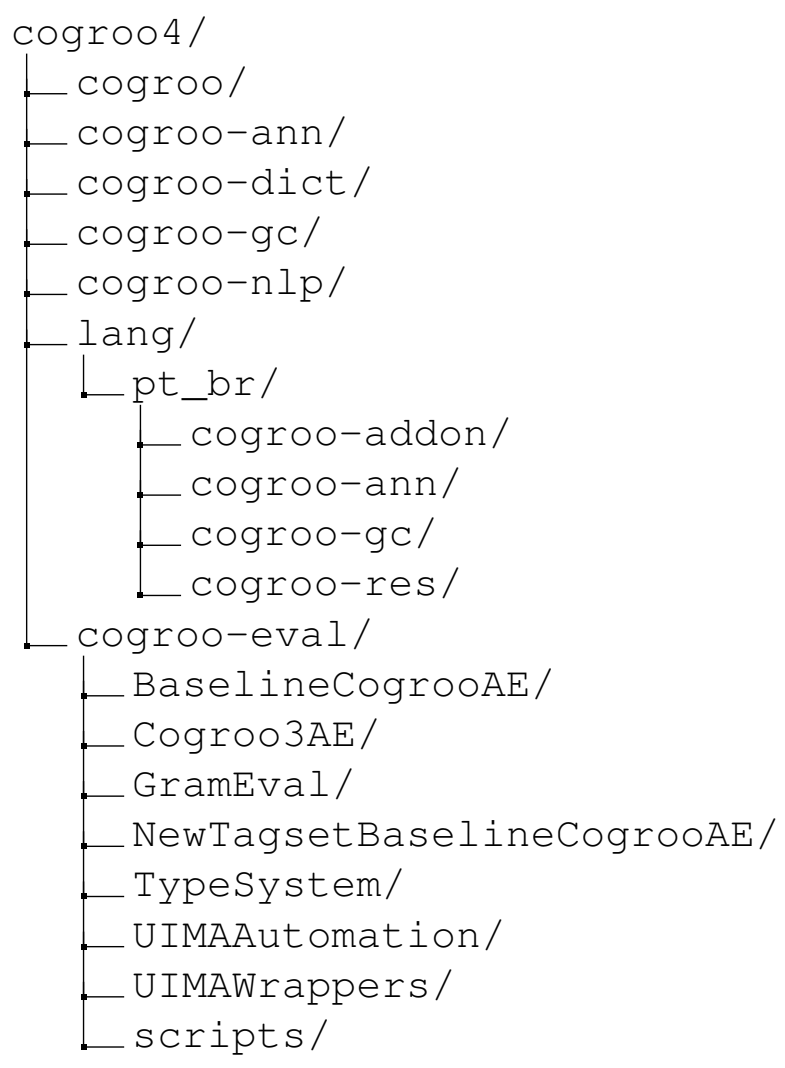

Table C.2: Directory structure of the source code.

cogroo-gc Implementations of standard checkers

lang/pt_br Language specific customizations

lang/pt_br/cogroo-res Compiled resources for Brazilian Portuguese, such as models and dictionaries.

lang/pt_br/cogroo-ann ANN language specific customizations.

lang/pt_br/cogroo-gc GC language specific customizations.

lang/pt_br/cogroo-addon The LibreOffice|OpenOffice grammar checker AddOn for Brazilian Portuguese.

cogroo-eval/BaselineCogrooAE An UIMA based project that can combine mixed versions of annotators, from sentence detector to name finder, and keep the CoGrOO 3.1.2 POS Tagger, Chunker and Shallow Parser modules.

cogroo-eval/NewTagsetBaselineCogrooAE An UIMA based project that can combine mixed version of annotators, but since it uses the new POS tagset, it disables annotators that can not work with the new tagset.

cogroo-eval/Cogroo3AE Bundles CoGrOO 3.1.2 as a UIMA annotator. 
cogroo-eval/GramEval Contains UIMA based corpus readers and the grammar checker evaluators, as well as tools to generate detailed reports.

cogroo-eval/TypeSystem A shared UIMA type system library used by CoGrOO evaluator projects.

cogroo-eval/UIMAAutomation Contains tools to automate execution of UIMA based software, such as a command line CPE runner and a tool to automatically install UIMA artifacts.

cogroo-eval/UIMAWrappers Contains UIMA wrappers to each of the CoGrOO analyzers.

cogroo-eval/scripts A number of Shell and Perl scripts that automate the execution of the evaluation tools.

\section{C.3 Runtime}

The following sections cover aspects related to use CoGrOO either as a NLP library or as a grammar-check library, as well as how to build the tools and try them from command line.

\section{C.3.1 Build}

To build the grammar checker, simply move to the main project aggregator and execute the Maven build command, as shown in Code 3:

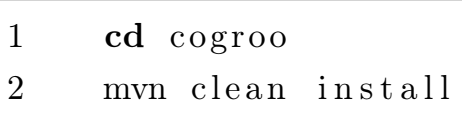

Code 3: Build the grammar checker using Maven.

The distributable product, named cogroo-addon-pt_br-4.0.0-SNAPSHOT.oxt, which can be installed in LibreOffice or OpenOffice, will be created at cogroo4/lang/pt_br/cogroo-addon/target/. Other distributables, like jar files, are available in each project's target folder.

\section{C.3.2 Executing from the Command Line}

It is possible to try the grammar checker from command line. The following command sequence shows how to do it. 
1 cd cogroo4/lang/pt_br/cogroo-gc

2 mvn $-\mathrm{e}-\mathrm{o}-\mathrm{q}$ exec : java "-Dexec.mainClass=org.cogroo.checker.

GrammarChecker" "-Dexec.args=pt_BR"

Code 4: Start the grammar checker from command line.

\section{C.3.3 CoGrOO as a NLP Library}

The easiest way to use CoGrOO as a library is by adding it as a dependency to a mavenized project. Add the configuration from Code 5 to the pom.xml.

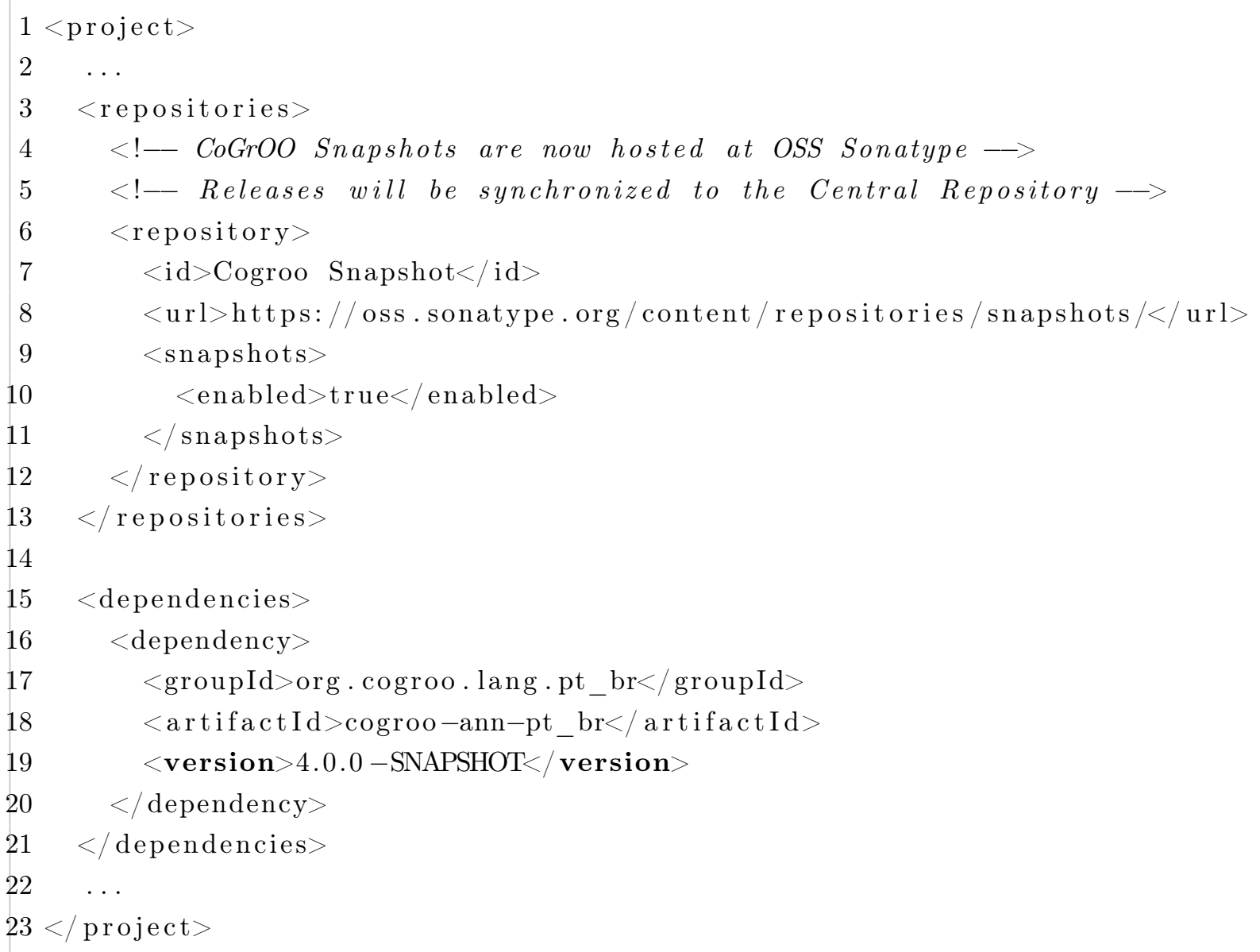

Code 5: Add CoGrOO Annotators as a dependency to your pom.xmI.

Code 6 show how it is easy to use the CoGrOO natural language processing API, from cogroo-ann project. Line 1 shows how to create a ComponentFactory, and Line 2 how to create the pipeline of annotators.

1 ComponentFactory factory=ComponentFactory.create (new Locale ("pt", "BR"));

2 AnalyzerI $\operatorname{cogroo}=$ factory $\cdot$ createPipe () ;

Code 6: Create the default pipeline of analyzers. 
Code 7 show how to analyze a text. First it creates a Document object and use the pipeline to annotate the document. After, it shows how to access the most common annotations, from sentence level to shallow parsing level.

$1 /$ The documentText is a String which can have multiple paragraphs

2 Document document $=$ new DocumentImpl(documentText);

3 cogroo.analyze (document);

$4 / /$ The document object is now annotated.

$5 /$ The following lines show how to access the annotations

6

7 for (Sentence sentence : document.getSentences ()) $\{/ /$ list of sentences

8 sentence.getStart (); sentence.getEnd ()$; / /$ the sentence starting and ending position

9 sentence.getText (); // the sentence text

10

$11 / /$ Tokens

12 for (Token token : sentence.getTokens ()) \{// list of tokens

13 token.getStart (); token.getEnd (); // the token start and end position

14 token.getLexeme (); // the lexeme of the token

15 token.getLemmas(); // the possible lemmas for the token and POS Tag

16 token.getPOSTag ()$; / /$ POS tag of the token

17 token.getFeatures (); // Features of the token, such as gender, number $18\}$ and tense

$$
\}
$$

\section{// Chunks}

for (Chunk chunk : sentence.getChunks()) \{// list of chunks chunk.getStart(); chunk.getEnd(); // the chunk start and end position chunk.getTag(); // the chunk tag \} chunk.getTokens(); // a list with the covered tokens

\section{Structure}

for (SyntacticChunk structure : sentence.getSyntacticChunks()) $\{/ /$ list of SyntacticChunks

29 structure.getStart(); structure.getEnd(); // the start and end position

30 structure.getTag (); // the structure tag

31 structure.getTokens (); // a list with the covered tokens

Code 7: Analyzing a document and accessing its annotations.

It is possible to customize the pipeline initialization customizing the models file. Code 8 exemplifies it by commenting on some annotators of the pipeline.

\section{C.3.4 CoGrOO as a Grammar Check Library}

To use CoGrOO as a grammar checker library is similar to the way it is used as a NLP library. Instead of adding cogroo-ann as a dependency in pom.xml, add cogroo-gc (Code 9). 
$1<$ languageConfiguration $\mathrm{xmlns}: \mathrm{jxb}=$ "http://java.sun.com/xml/ns/jaxb" xmlns:xsi=" http://www.w3.org/2001/XMLSchema-instance"

xsi:noNamespaceSchemaLocation=" languageConfiguration . xsd" $>$

$<$ locale $>$ pt_BR $</$ locale $>$

$<$ model type=" sentenceDetector " $>/$ models $/$ pt - sent. model $</$ model $>$

$<$ model type="tokenizer" $>/$ models $/$ pt - tok $\cdot \operatorname{model}</$ model $>$

$<$ model type $=$ "nameFinder" $>/$ models $/$ pt - prop. model $</$ model $>$

$<$ model type $=$ " contractionFinder $">/$ models $/$ pt - con. model $</$ model $>$

$<$ model type $="$ posTagger $">/$ models $/$ pt - pos. model $</$ model $>$

$<!-<$ model type=" featurizer " $>/$ models $/$ pt - feat. model $</$ model $>$

$<$ model type $="$ chunker" $>/$ models $/$ pt - chunk. model $</$ model $>$

$<$ model type $="$ headFinder $">/$ models $/ p t-h f$. model $</$ model $>$

$<$ model type $=$ "shallow Parser" $>/$ models $/ p t-s p$. model $</$ model $>->$

$<$ pipe $>$

$<$ analyzer $>$ sentenceDetector $</$ analyzer $>$

$<$ analyzer $>$ tokenizer $</$ analyzer $>$

$<$ analyzer $>$ nameFinder $</$ analyzer $>$

$<$ analyzer $>$ contraction Finder $</$ analyzer $>$

$<$ analyzer $>$ posTagger $</$ analyzer $>$

$<!-<$ analyzer $>$ featurizer $</$ analyzer $>$

$<$ analyzer $>$ lemmatizer $</$ analyzer $>$

$<$ analyzer $>$ chunker $</$ analyzer $>$

$<$ analyzer $>$ headFinder $</$ analyzer $>$

$<$ analyzer $>$ shallowParser $</$ analyzer $>->$

$</$ pipe $>$

$28</$ languageConfiguration $>$

Code 8: Example of modified models.xml. Some lines were commented to remove annotators from the pipeline.

$1<$ project $>$

$2 \quad \ldots$

$3<$ repositories $>$

$4<!--$ CoGrOO Snapshots are now hosted at OSS Sonatype $\rightarrow$

$5 \quad<!--$ Releases will be synchronized to the Central Repository $\longrightarrow$

$6<$ repository $>$

$7 \quad<\mathrm{id}>$ Cogroo Snapshot $</ \mathrm{id}>$

$8<$ url $>$ https://oss.sonatype.org/content/repositories/snapshots/ $</$ url $>$

$9<$ snapshots $>$

$10<$ enabled $>$ true $</$ enabled $>$

$</$ snapshots $>$

$</$ repository $>$

$</$ repositories $>$

$<$ dependencies $>$

$<$ dependency $>$

$<$ groupId $>$ org.cogroo.lang.pt_br $</$ groupId $>$

$<$ artifactId $>$ cogroo - gc - pt_br $</$ artifactId $>$

$<$ version $>4.0 .0-$ SNAPSHOT $</$ version $>$

$</$ dependency $>$

$</$ dependencies $>$

$21</$ project $>$

Code 9: Add CoGrOO Grammar Checker as a dependency to your pom.xmI. 
Code 10 show how to easily create a grammar checker using CoGrOO API. This time it is required to manually add the GrammarCheckerAnalyzer to the pipeline.

$1 / /$ create the grammar checker analyzer

2 GrammarCheckerAnalyzer gca = new GrammarCheckerAnalyzer ();

$3 / /$ initialize other analyzers and get the pipeline. The cast is necessary because we need to add gca to the pipeline

4 ComponentFactory factory=ComponentFactory.create (new Locale ("pt", "BR"));

5 Pipe $\operatorname{cogroo}=($ Pipe) factory createPipe () ;

6 cogroo.add (gca);

Code 10: Create the default pipeline of analyzers and add the grammar checker to it.

Code 11 show how to find grammar errors of a text. First it creates a CheckDocument object and use the pipeline to annotate the document. To retrieve the mistake list one can simply call document.getMistakes ().

$1 / /$ create a CheckDocument

2 CheckDocument document $=$ new CheckDocument () ;

3 document. setText (text);

4 // analyze it

5 cogrooPipe. analyze (document);

6

$7 / /$ get the results using document.getMistakes ()

8 List $<$ Mistake $>$ mistakes $=$ document. getMistakes () ;

Code 11: Analyzing a document and accessing its annotations.

Many other instructions and examples are available in the Wiki pages of the project ${ }^{3}$.

\section{C.4 Evaluation}

The following sections cover the tools used to evaluate the NLP modules and the grammar checker.

To setup the development environment one should install Apache UIMA 2.3.x, setup the environment variables as described in Code 12 and organize the CORPUS_ROOT folder as described in Table C.3.

\section{C.4.1 Executing 10-Fold Cross-Validation}

This section shows how to reproduce the 10-fold cross-validation experiments presented in this work. An automation script executes a configurable set of experiments and create a report. Another set of scripts are used to create the ${ }^{A} \mathrm{~T}_{\mathrm{E}} \mathrm{X}$ tables and graphics used.

\footnotetext{
${ }^{3}$ CoGrOO Wiki, http://ccsl.ime.usp.br/redmine/projects/cogroo/wiki, last accessed on 12/28/2012
} 
1 \# COGROO_3 points to the root of CoGrOO 3 source code

2 export COGROO_3 $=$ / workspace / cogroo 3

3 \# MODEL_ROOT points to a folder were models are created

4 export $\overline{M O D E L} \mathrm{ROOT}=\sim /$ workspace $/$ MODELS

5 \# REPO_ROOT points to a folder were UIMA artifacts are deployed

6 export REPO_ROOT= $/$ workspace $/ \mathrm{REPO}$

7 \# CORPUS_ROOT points to a folder were to find the training and evaluating corpora

8 export CORPUS_ROOT $=\sim /$ workspace $/$ CORPUS

9 \# UIMA_HOME points to the folder were UIMA is installed

10 export UIMA_HOME $=\sim /$ programs/apache-uima

Code 12: Development environment variables.

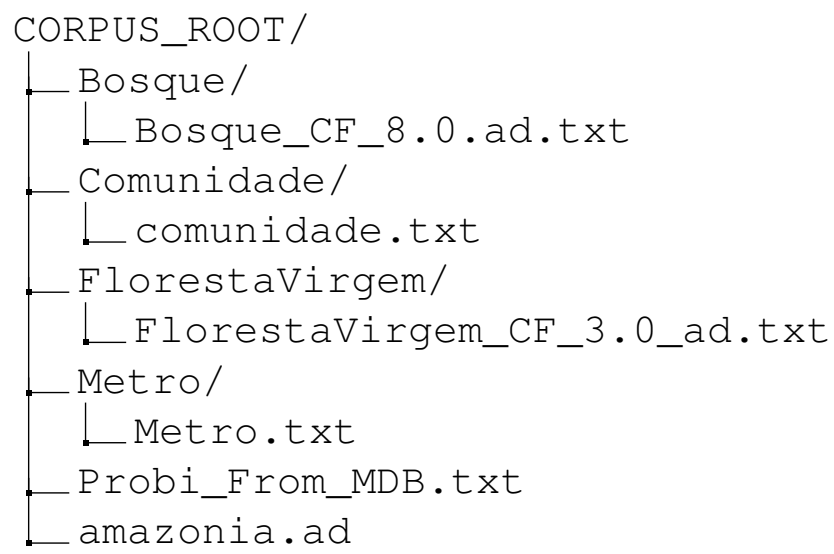

Table C.3: Files structure of the CORPUS_ROOT folder.

Code 13 shows how to execute the evaluation. Evaluating a module can take several hours. Each report can be found in the folder cogroo4/lang/pt_br/cogroo-res/eval. Table C.4 shows a snippet of the POS Tagger evaluation report, which includes information such as number of predicates, evaluation and training time, model size and the resultant accuracy.

\begin{tabular}{|c|c|c|c|c|c|}
\hline cutoff & Predicates & Eval Time & Training Time & Model Size & Accuracy \\
\hline 0 & 88411 & 287.259297 & 38.44192 & 4546082 & 0.9629057187017002 \\
2 & 37711 & 236.782653 & 31.865341 & 4478964 & 0.9604473706629021 \\
4 & 18820 & 209.373552 & 28.784877 & 4426564 & 0.9575995813506311 \\
8 & 9756 & 188.651653 & 26.089485 & 4377880 & 0.9545449013618275 \\
16 & 5218 & 176.241469 & 24.686258 & 4335604 & 0.9489223429760615 \\
32 & 2881 & 165.931604 & 23.63556 & 4300206 & 0.9396122625320985 \\
64 & 1513 & 157.191878 & 22.554011 & 4271006 & 0.933332521997347 \\
128 & 807 & 148.502101 & 21.710632 & 4248829 & 0.9275030729350485 \\
256 & 487 & 141.374345 & 20.818145 & 4236942 & 0.9114994706032689 \\
512 & 291 & 134.232848 & 20.153857 & 4227070 & 0.9077754384256836 \\
\hline
\end{tabular}

Table C.4: Example result table for 10-fold cross validation evaluation.

Other scripts are available to consume the generated reports, for example convert_to_r.pl 
1 \# go to pt_BR resources project

2 cd cogroo $4 /$ lang/pt_br/cogroo-res

3 \# evaluate Sentence Detector

4 sh scripts/sd_run.sh

5 \# evaluate Tokenizer

6 sh scripts/tok_run.sh

7 \# evaluate Contraction Finder

8 sh scripts/con_run.sh

9 \# evaluate Proper Name Finder

$10 \mathrm{sh}$ scripts/prop_run.sh

11 \# evaluate POS Tagger

12 sh scripts/tagger_run.sh

13 \# evaluate Featurizer

$14 \mathrm{sh}$ scripts/featurizer_run.sh

15 \# evaluate Chunker

16 sh scripts/chunker_run.sh

17 \# evaluate Chunker Head Finder

18 sh scripts/hf run.sh

19 \# evaluate Shallow Parser

$20 \mathrm{sh}$ scripts/sp_run.sh

Code 13: Produces detailed 10-fold cross-validation reports. Each evaluation can take several hours.

is a Perl script which takes an experiment result and generates a $\mathrm{R}$ script to generate the graphics used in this work, for example the one in Figure 6.6.

\section{C.4.2 Grammar Checker Evaluation}

Code 14 exemplifies how to execute the grammar checker evaluation. In this case, it compares the effectiveness of CoGrOO 3.1.2 and the version proposed by this work. The script will create a folder named eval and a number of reports. The directory structure of the reports is detailed in Table C.5, and each report is described below.

1 \# go to the scripts directory of eval project

2 cd cogroo-eval/scripts

3 perl evaluate_gc.pl sp sp-baseline

Code 14: Sample execution of the grammar checker evaluation.

details.txt Lists all false positives, false negatives and true positives.

fmeasure.html A detailed visual report which details how the grammar checker performed in each category.

fmeasure.txt The same information from fmeasure.html, but in pure text format

diff.txt A comparison between false positives, false negatives and true positives of this version and the baseline.

diff-baseline.txt The same report from diff.txt, but with annotations from baseline. 


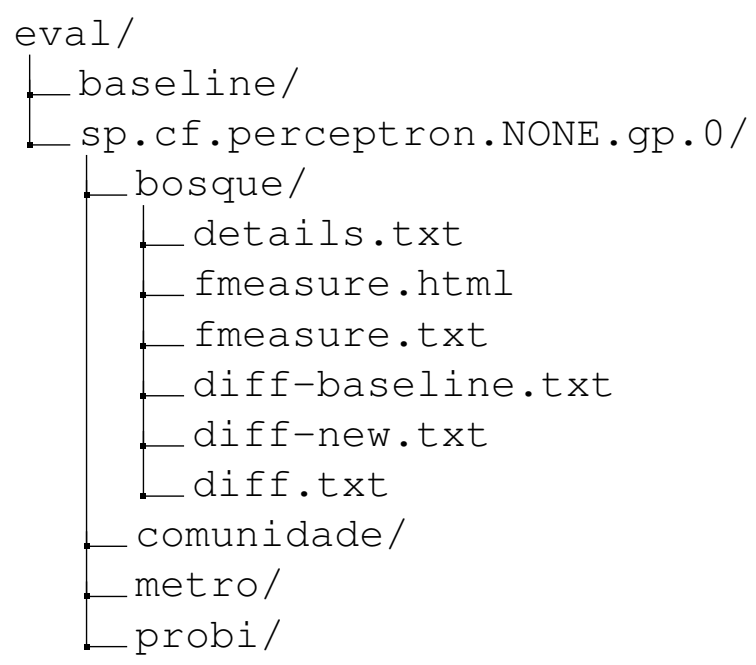

Table C.5: Report files generated by evaluate_gc.pl.

diff-new.txt The same report from diff.txt, but with annotations from this version.

The following figures illustrate the reports. Figure C.2 shows how useful the diff files are in understanding the annotation changes between versions. Figures C.3, C.4, C.5, C.6, C.7, C.8 and C.9 are part of the HTML which can be used to understand bottlenecks of the system.

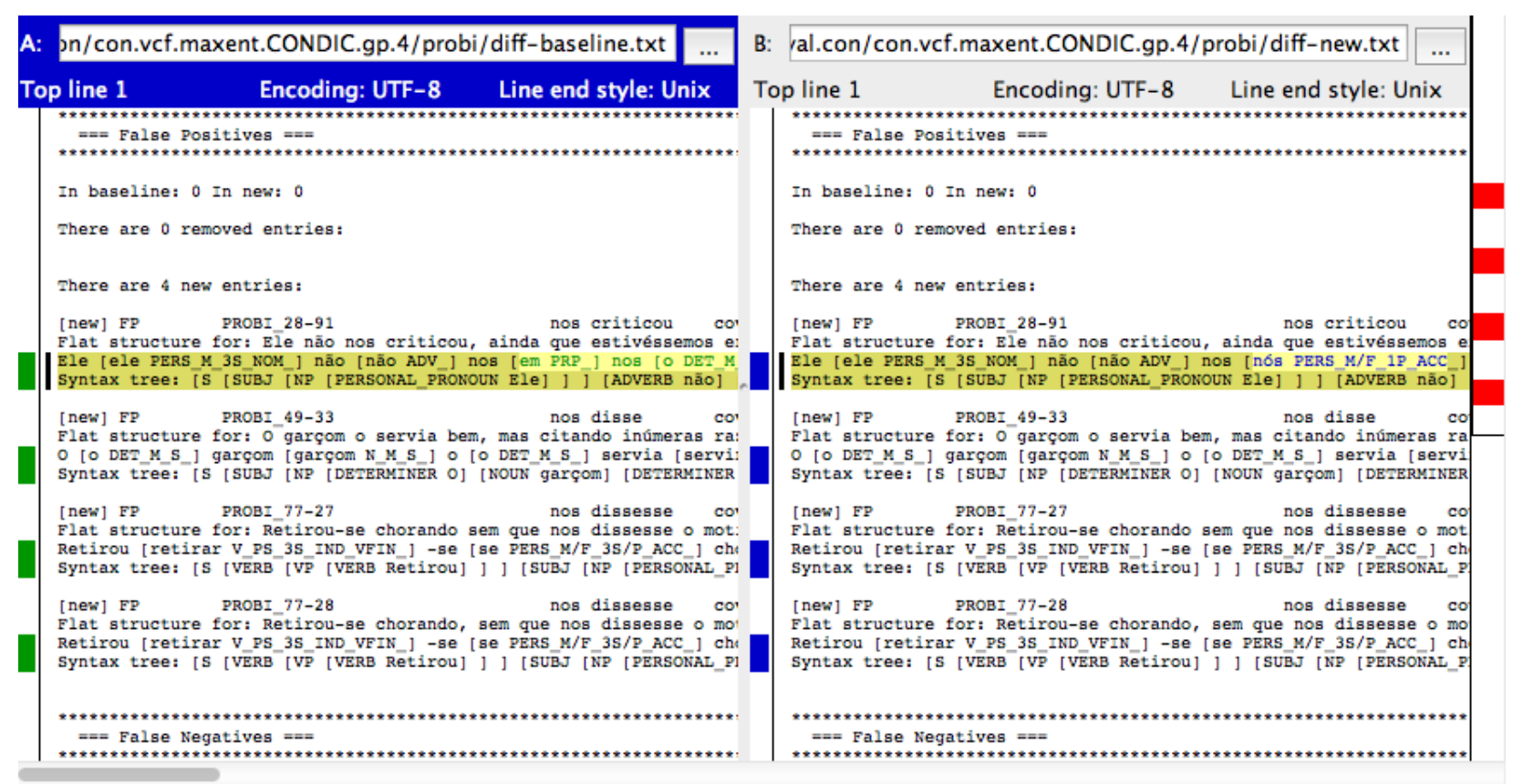

Figure C.2: Comparing a sample diff-baseline.txt and a diff-ne.txt in KDiff. The left pane shows the annotations from CoGrOO 3.1.2, which erroneously annotated nos as a contraction. The right pane shows the new version which classified it as a pronoun. 


\section{Visão Geral}

\section{Resumo}

The program counted 11625 sentences with 2616 grammar errors according to corpus. CoGrOO found 628 grammar errors of which 377 were correct. The evaluation presents the precision, recall and F1 rates, both overall (TOTAL) and for each of the grammar error categories in the data. Only grammar error categories that are present in the corpus data or in the predicted output will be mentioned in the evaluation overview. The zero scores for some categories can be explained by the fact that there are some grammar errors in this data but none of them was detected by CoGrOO.

Passe o mouse para uma descrição da categoria:

abr, ace, adj, adv, aha, ali, arc, cap, cjc, cli, cmt, con, con|cov, cop, cop|pro, cov, cov|ver, cra, det, esp, est, ger, lex, mal, mec, mor, nol, ond, ort, par, pre, pro, prq, ptn, ptp, reg, ren, rep, sem, ver.

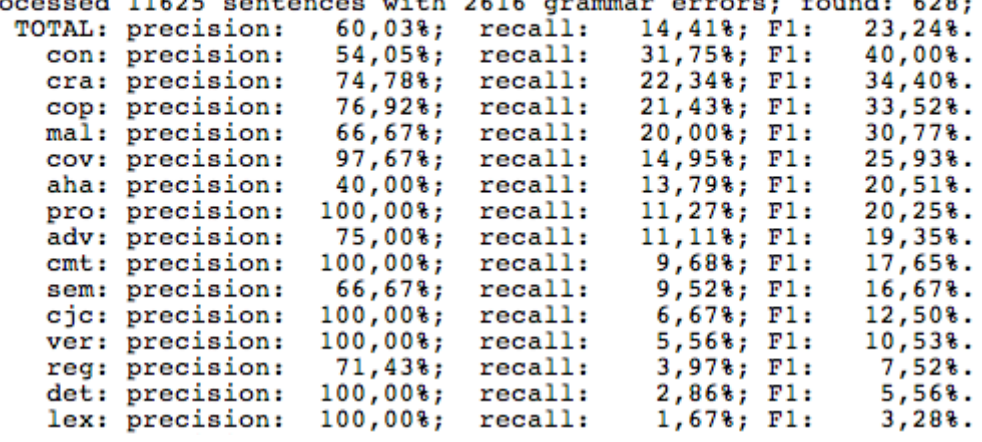

Figure C.3: Sample report - effectiveness overview in terms of $F_{1}$ for each error category.

\section{Distribuição de erros gramaticais no corpus}

Neste gráfico vemos a distribuição de erros no Corpus . Os erros foram manualmente anotados.

\section{Distribuição de Erros Gramaticais no Corpus.}

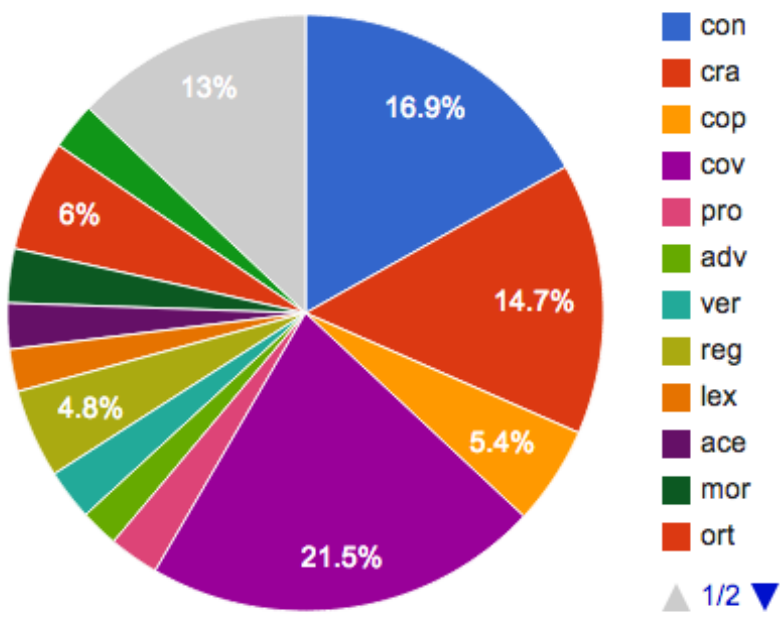

Figure C.4: Sample report - distribution of grammar error categories in the corpus. 


\section{Desempenho do CoGrOO no Corpus}

Este gráfico mostra o desempenho do CoGrOO no corpus. Precisão é a proporção de itens selecionados corretamente pelo corretor gramatical: $\mathrm{P}=\mathrm{TP} / \mathrm{TP}+\mathrm{FP}$. Cobertura é a proporção de erros gramaticais do corpus que foram corretamente marcados pelo corretor gramatical: R=TP/Target

Nota: categorias em que o CoGrOO não pontuou foram omitidas.

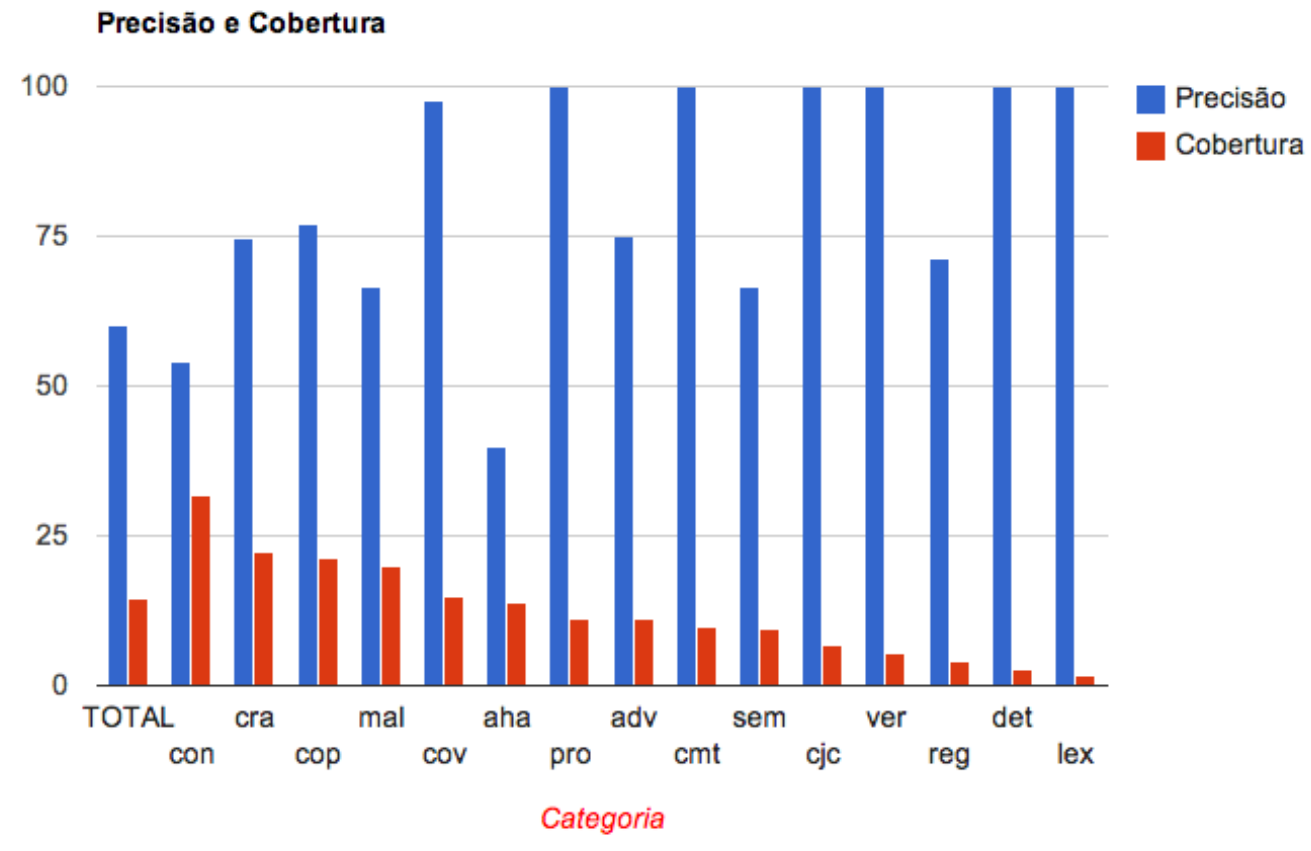

Figure C.5: Sample report - CoGrOO effectiveness in each error category.

\section{Conjuntos Target e Selected no Corpus}

Existem 2616 erros gramaticais no córpus (target). O CoGrOO marcou 628 erros (selected). 377 elementos estão na interseção (tp).

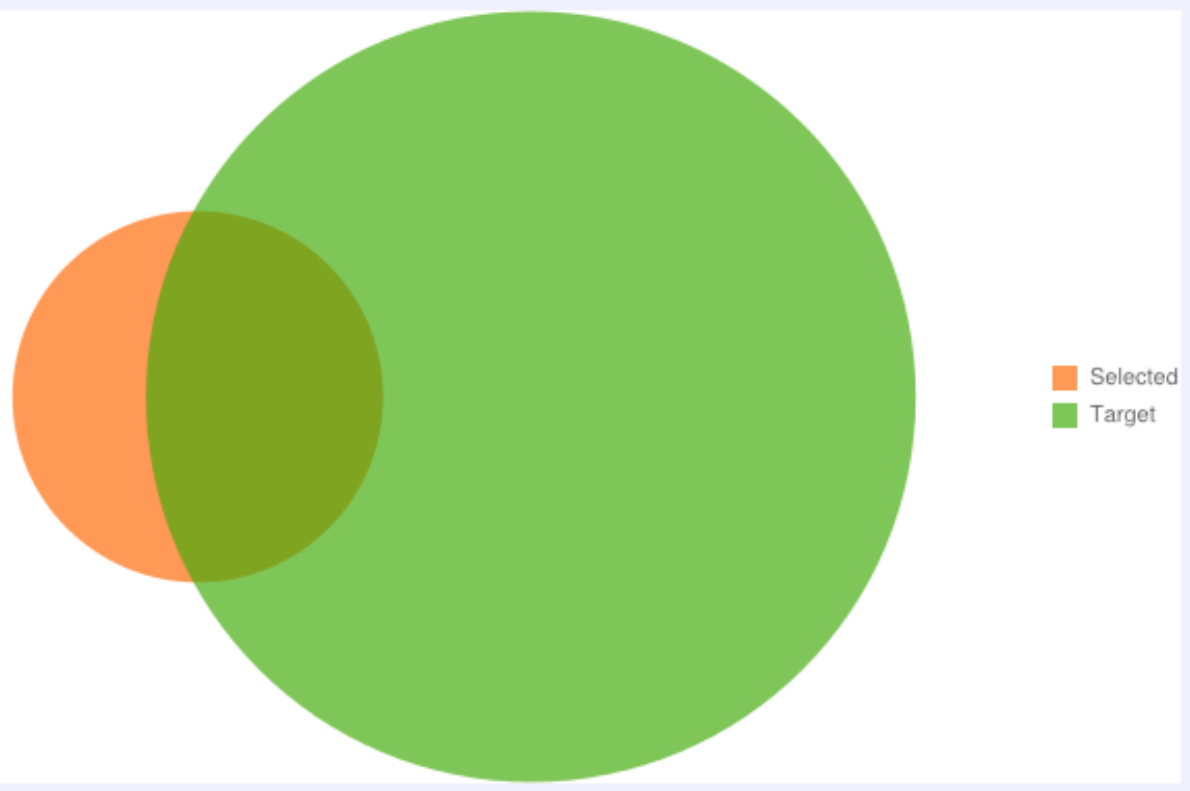

Figure C.6: Sample report - Venn diagram comparing the target and the selected errors. 


\section{Falsos Positivos por categoria}

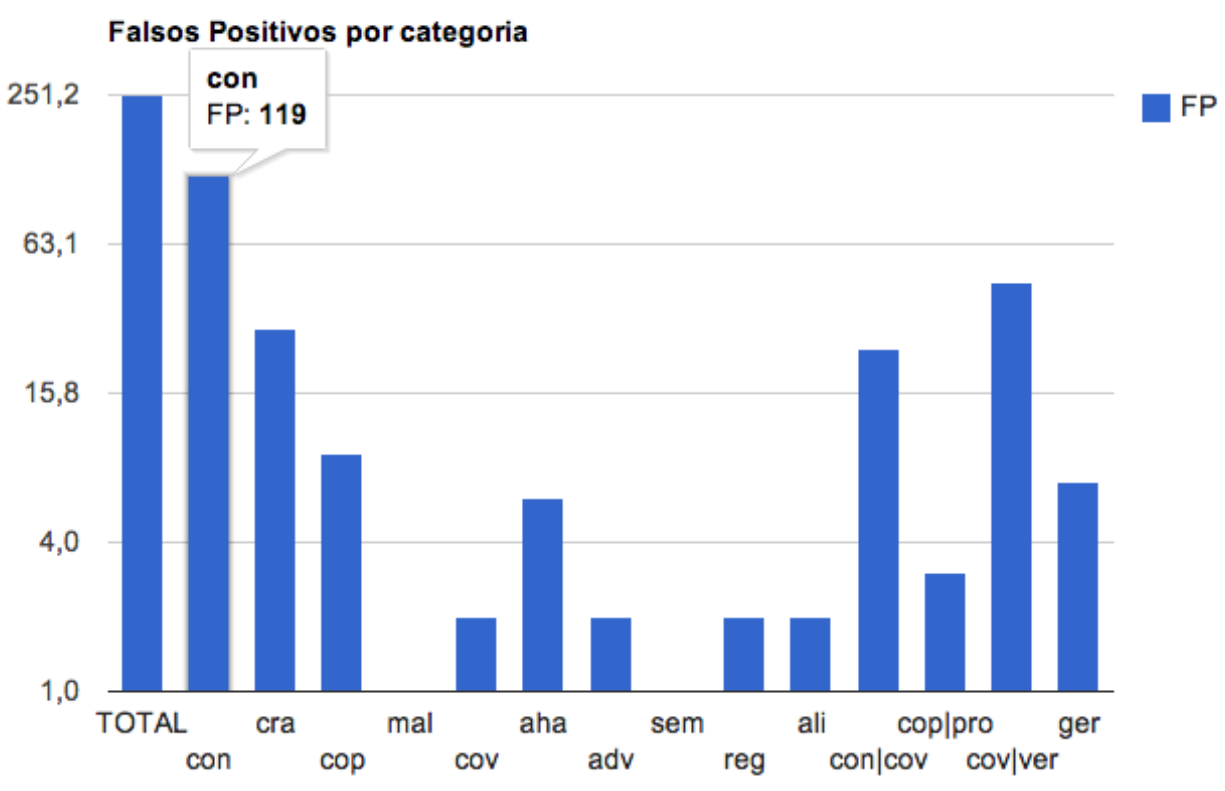

Figure C.7: Sample report - Number of false positives for each category.

\section{Categorias descobertas}

As seguintes categorias estão presentes no córpus mas o CoGrOO não possui regras que as contemple: abr, ace, adj, arc, cap, cjc, cli, det, est, lex, mec, mor, nol, ond, ort, par, pre, prq, ptp, rep.

\section{Proporção de erros com categorias cobertas}

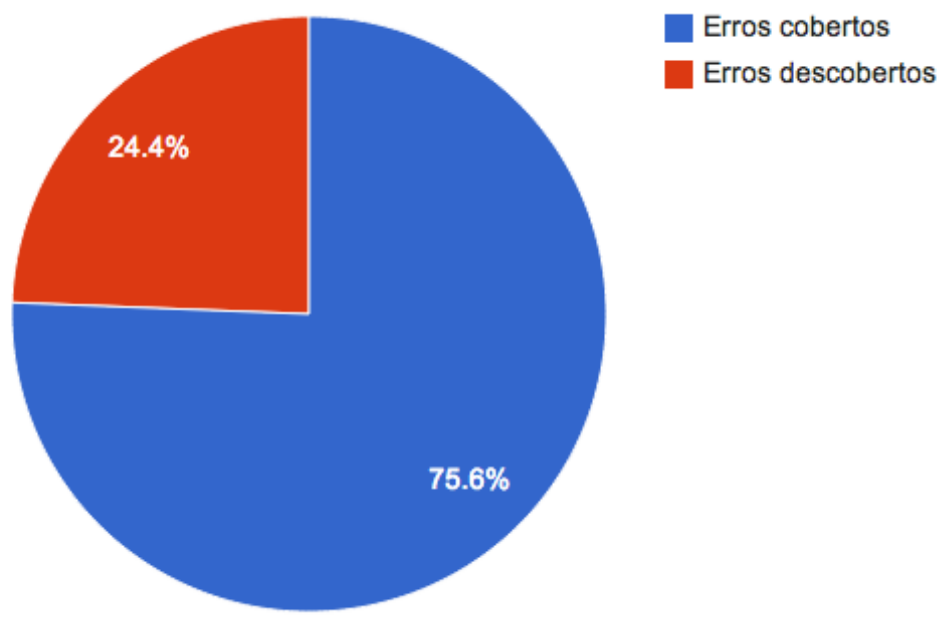

Figure C.8: Sample report - Compares the number of samples in which error category is covered by CoGrOO. 


\section{Detalhes das categorias}

con - Conjuntos Target e Selected no Corpus

target: 441 ; selected: 259 ; tp: 140 .

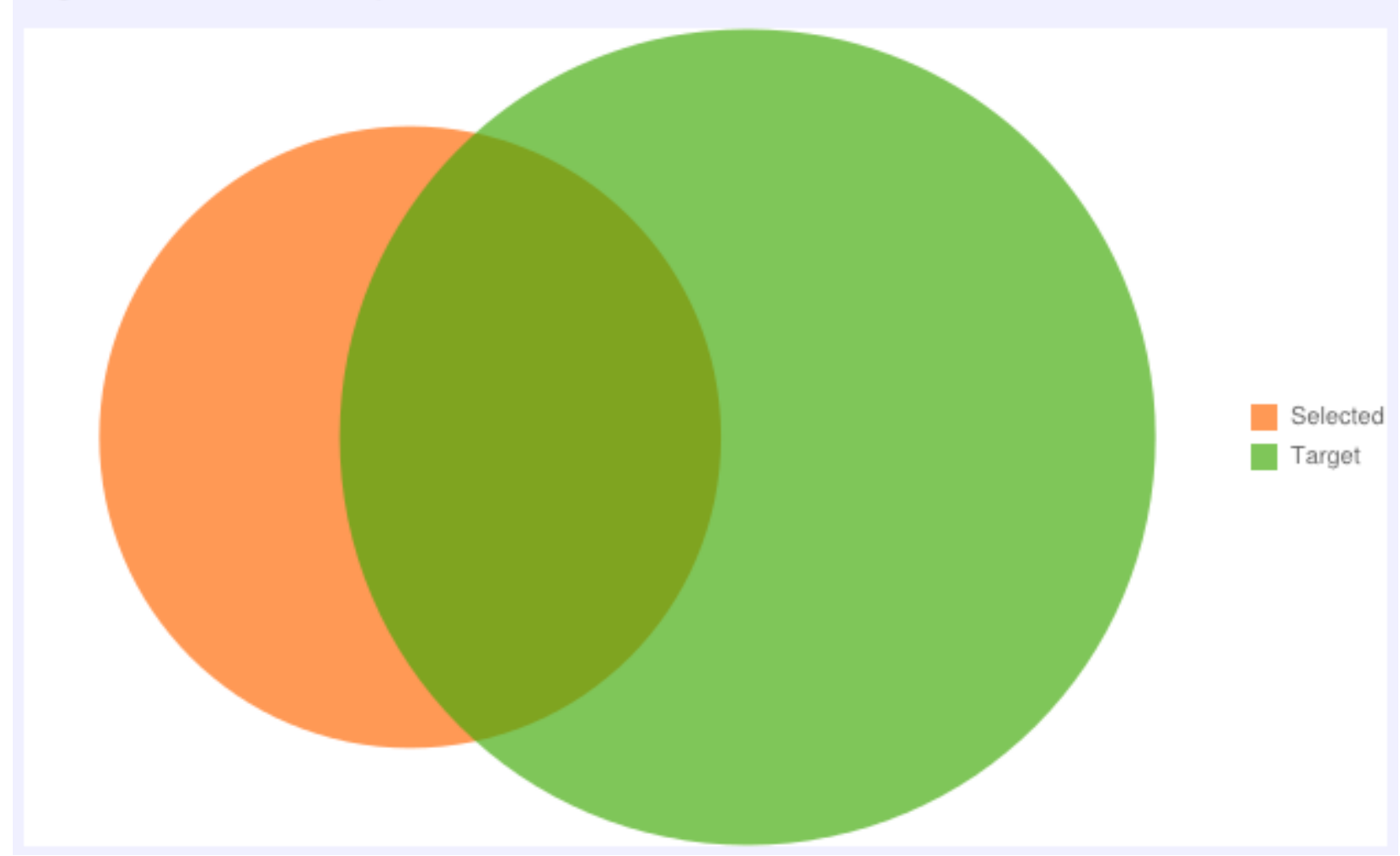

Figure C.9: Sample report - Venn diagram which compares the target and the selected for each error category. 


\section{Bibliography}

Afonso(2006) Susana Afonso. Árvores deitadas: Descrição do formato e descrição das opções de análise na floresta sintá(c)tica. Technical report, Floresta Sintática. 14, 63

Afonso(2003) Susana Afonso. A floresta sintá(c)tica como recurso. Documentaçao disponıvel na Linguateca. 2, 12

Afonso et al.(2001) Susana Afonso, Eckhard Bick, and Ana Raquel Marchi. Critérios de separação de sentenças/frases, fevereiro 2001. URL http://www.linguateca.pt/floresta/ doc/CriteriosSeparacao.html. 51, 134

Alfenas and Pereira-Barretto(2012) D. A. Alfenas and Marcos Ribeiro Pereira-Barretto. Adaptability in sociable robots: proposal for a dialog manager. Technical report, Polytechnic School, São Paulo University. 138

Almeida(2011) João Almeida. Natura project, natural language processing group. http: //natura.di.uminho.pt/wiki/doku.php?id=projectonatura, January 2011. 12

Almeida and Pinto(1994) José João Almeida and Ulisses Pinto. Jspell: um módulo para análise léxica genérica de linguagem natural. Actas do X Encontro da Associaçao Portuguesa de Lingurstica, pages 1-15. 12

Baldridge et al.(2002) Jason Baldridge, Thomas Morton, and Gann Bierner. The OpenNLP Maximum Entropy Package. Technical report, SourceForge, Apache. 10

Bick(2000) Eckhard Bick. The Parsing System Palavras: Automatic Grammatical Analysis of Portuguese in a Constraint Grammar Framework. PhD thesis, Aarhus University, Aarhus, Denmark. 6, 8, 9

BNC(2003) BNC. British national corpus. http://www.hcu.ox.ac.uk/BNC, June 2003. 17

Brill(1992) Eric Brill. A simple rule-based part of speech tagger. In Proceedings of ANLP92, 3rd Conference on Applied Natural Language Processing. 8, 9, 17

Chiarelli et al.(1990) Carlos Chiarelli et al. Portuguese language orthographic agreement of 1990, December 1990. 13, 19

Collins(2002) Michael Collins. Discriminative training methods for hidden markov models: Theory and experiments with perceptron algorithms. In Proceedings of the ACL-02 conference on Empirical methods in natural language processing-Volume 10, pages 1-8. Association for Computational Linguistics. 12, 53, 56

Fernandes et al.(2010) Eraldo Fernandes, Cicero Santos, and Ruy Luiz Milidiú. A machine learning approach to portuguese clause identification. In PROPOR 2010: International Conference on Computational Processing of the Portuguese Language, 2010, Porto Alegre. $112,115,119,120$ 
Finger and Alves(1999) Marcelo Finger and Carlos Alves. Etiquetagem do português clássico baseada em córpora. In PROPOR 1999: IV Encontro para Processamento Computacional da Lingua Portuguesa Escrita e Falada. 9

Flesch(1948) Rudolf Flesch. A new readability yardstick. Journal of Applied Psychology, 32:221-233. 16

Galves and Faria(2010) Charlotte Galves and Pablo Faria. Tycho brahe parsed corpus of historical portuguese. http://www.tycho.iel.unicamp.br, 2010. 9

Jaynes(1957) E. T. Jaynes. Information theory and statistical mechanics. Phys. Rev., 106 (4):620-630. doi: 10.1103/PhysRev.106.620. 10

Jurafsky et al.(2000) Daniel Jurafsky, James H. Martin, Andrew Kehler, Keith Vander Linden, and Nigel Ward. Speech and language processing: An introduction to natural language processing, computational linguistics, and speech recognition, volume 163. MIT Press. 5, 10

Karlsson et al.(1995) Fred Karlsson, Atro Voutilainen, and Juha Heikkilae. Constraint Grammar: a language-independent system for parsing unrestricted text. Walter de Gruyter. 6,9

Kepler(2005) Fabio Natanael Kepler. Um etiquetador morfo-sintático baseado em cadeias de markov de tamanho variavel. Master's thesis, Institute of Mathematics and Statistics of the University of São Paulo. 8, 9

Kinoshita et al.(2006) Jorge Kinoshita, Lais Nascimento Salvador, and Carlos Eduardo Dantas de Menezes. Cogroo: a brazilian-portuguese grammar checker based on the cetenfolha. In Proceedings LREC 2006. 2

Kinoshita et al.(2007) Jorge Kinoshita, Lais Nascimento Salvador, Carlos Eduardo Dantas Menezes, and William Daniel Colen de Moura Silva. CoGrOO-An OpenOffice Grammar Checker. In Intelligent Systems Design and Applications, 200\%. ISDA 200\%. Seventh International Conference on, pages 525-530. IEEE. 19, 25

Luz(2012) Fabiano Ferreira Luz. Consulta à ontologias em língua portuguesa através do português controlado. Master's thesis, Computer Science Department, University of São Paulo. 137

LX-CENTER(2011) LX-CENTER. Language resources and technology for portuguese. http://lxcenter.di.fc.ul.pt/home/en/index.html, January 2011. 10

Manning and Schütze(1999) Chris Manning and Hinrich Schütze. Foundations of statistical natural language processing, volume 59. MIT Press. 6, 7

Martins(2002) Ronaldo Martins. Probi: um corpus de teste para o revisor gramatical regra. http://www.nilc.icmc.usp.br/nilc/download/NILC-TR-02-10.zip, February 2002. $2,15,145$

Matte et al.(2012) Ana Cristina Fricke Matte, Rubens Takiguti Ribeiro, William Daniel Colen de Moura Silva, and Hugo Leonardo Canalli. Dadossemiotica: coleta e processamento de análises semióticas de texto escrito. In WSL Workshop Internacional de Software Livre. 138 
Menezes et al.(2006) Carlos Eduardo Dantas Menezes, Fabio Wang Gusukuma, and Sueli Uliano. Uma análise do cogroo, um corretor gramatical acoplável ao openoffice. http: //www.pcs.usp.br $/{ }^{\sim}$ cogroo/papers/analise-cogroo-corpus-metro.html, February 2006. 2, 15

Metro(2011) Metro. http://www.metro.sp.gov.br, January 2011. 15

Moura(2011) Raimundo Moura. Vero, the brazilian portuguese spell checker. http://www. broffice.org/verortografico, January 2011. 13

Naber(2003) Daniel Naber. A rule-based style and grammar checker. PhD thesis, Faculty of Technology, Bielefeld University. 3, 17

Nemeth(2011a) Laszlo Nemeth. Hunspell spell checker and morphological analyzer designed. http://hunspell.sourceforge.net/, January 2011a. 13, 17

Nemeth(2011b) Laszlo Nemeth. Lightproof, a light weight grammar checker. http:// libreoffice.hu/2011/12/08/grammar-checking-in-libreoffice/, January 2011b. 17

Nunes and Oliveira(2000) Maria Nunes and Osvaldo Oliveira. O processo de desenvolvimento do revisor gramatical regra. Technical report, Núcleo Interinstitucional de Linguística Computacional - NILC, ICMC/USP. 16

Oleynik(2012) Michel Oleynik. Extração de informações de narrativas clínicas. Master's thesis, Computer Science Department, University of São Paulo. 137

Oleynik et al.(2010) Michel Oleynik, P. Nohama, PS Cancian, and S. Schulz. Performance Analysis of a POS Tagger applied to Discharge Summaries in Portuguese. Studies in health technology and informatics, 160:959. ISSN 0926-9630. 6, 137

Oliveira et al.(2012) Lucas Emanuel Silva Oliveira, Claudia Maria Cabral Moro, Andréia Cristina Souza, Percy Nohama, and Pindaro Secco Cancian. Identificação de continuidade de cuidado em sumários de alta hospitalar. In XIII Congresso Brasileiro em Informática em Saúde. 138

Ratnaparkhi(1996) Adwait Ratnaparkhi. A maximum entropy model for part-of-speech tagging. In Proceedings of the conference on empirical methods in natural language processing, volume 1, pages 133-142. 10, 20

Ratnaparkhi(1998) Adwait Ratnaparkhi. Maximum entropy models for natural language ambiguity resolution. PhD thesis, University of Pennsylvania. 51, 56, 87

Sang and Buchholz(2000) Erik F. Tjong Kim Sang and Sabine Buchholz. Introduction to the conll-2000 shared task: Chunking. In Proceedings of the 2nd workshop on Learning language in logic and the 4 th conference on Computational natural language learningVolume 7, pages 127-132. Association for Computational Linguistics. 116

Seidel(2012) Wesley Seidel. Reconhecimento de entidades mencionadas em português utilizando aprendizado de máquina. Master's thesis, Computer Science Department, University of São Paulo. 77

Sha and Pereira(2003) Fei Sha and Fernando Pereira. Shallow parsing with conditional random fields. In Proceedings of the 2003 Conference of the North American Chapter of the Association for Computational Linguistics on Human Language Technology-Volume 1, pages 134-141. Association for Computational Linguistics. 112, 116, 119 
Silva(2007) João Silva. Shallow processing of Portuguese: From sentence chunking to nominal lemmatization. Master's thesis, Department of Informatics, University of Lisbon. $5,6,10,23,57,68,103,107,134$

Silva et al.(2010) William Daniel Colen Moura Silva, Marcelo Finger, and Carlos Eduardo Dantas Menezes. Open text annotators using apache uima. In PROPOR 2010: International Conference on Computational Processing of the Portuguese Language, 2010, Porto Alegre. 19, 28, 137

Souza(2012) Andreia Cristina Souza. Identificação do Conteúdo Padronizado do Sumário de Alta. Master's thesis, PUCPR. 137

Torres(2012) Carlos Eduardo Atencio Torres. Uso de informação linguística e análise de conceitos formais no aprendizado de ontologias. Master's thesis, Computer Science Department, University of São Paulo. 137

Woods(1970) William Aaron Woods. Transition networks grammars for natural language analysis. CACM, Special Issue on Computational Linguistics, 13:591-606. 6, 16 